\title{
The Advantages Of Paramagnetic NMR
}

\author{
Dissertation \\ for the award of the degree \\ Doctor rerum naturalium \\ of the University of Göttingen
}

within the doctoral program Physics of Biological and Complex Systems

of the Georg-August University School of Science (GAUSS)

submitted by

Florian Siepel

from Neustadt a. Rbge

Göttingen 2013 


\section{Thesis committee}

Prof. Dr. Christian Griesinger

NMR based Structural Biology, Max Planck Institute for Biophysical Chemistry, Göttingen

Prof. Dr. Jörg Enderlein

III. Institute of Physics, University of Göttingen

Prof. Dr. Andreas Janshoff

Institute for Physical Chemistry, University of Göttingen

\section{Members of the Examination Board}

Referee:

Prof. Dr. Christian Griesinger

NMR based Structural Biology, Max Planck Institute for Biophysical Chemistry, Göttingen

$2^{\text {nd }}$ Referee:

Prof. Dr. Jörg Enderlein

III. Institute of Physics, University of Göttingen

\section{Further Members of the Examination Board}

Prof. Dr. Andreas Janshoff

Institute for Physical Chemistry, University of Göttingen

Prof. Dr. Marina Bennati

Electron Spin Resonance Spectroscopy, Max Planck Institute for Biophysical Chemistry, Göttingen

Dr. Claudia Höbartner

Nucleic Acid Chemistry, Max Planck Institute for Biophysical Chemistry, Göttingen

Dr. Adam Lange

NMR based Structural Biology, Max Planck Institute for Biophysical Chemistry, Göttingen

Date of oral examination: 28.10 .2013 


\section{Affidavit}

I hereby declare that I wrote this thesis The Advantages Of Paramagnetic NMR on my own and without the use of any other than the cited sources and tools.

Place, Date

Signature 

Climb, climb, climb! 



\section{Acknowledgments}

First of all, I'd like to thank my doctoral advisor Prof. Griesinger who gave me the oppertunity and the freedom to work on two interesting but different topics. He was always contactable and had a quick and informative answer to all of my many questions.

I would also like to thank my thesis committee members, Prof. Enderlein and Prof. Janshoff. Their input in the committee meetings was of great value and led to very useful discussions and developements.

I thank the other members of the examination board, Prof. Bennati, Dr. Höbartner and Dr. Lange, that they are willing to join my oral examination.

Thanks to Dr. Andrei Leonov whose knowledge about chemistry and his always granted help had a huge impact on this work. I thank Kerstin Overkamp and Gerhard Wolf for many HPLC purifications of the DNA samples and the measurements of the mass spectra. Thanks to Dr. Dirk Bockelmann for all the help with the computers and software.

My specials thanks to all readers of this thesis, especially Eibe Dücker.

Thanks for the great atmosphere in the lab to Sebastian Täubert, Eibe Dücker, Manuel Schmidt, Dr. Nina Schützenmeister, Dr. Fabian Peters and Ruth Linder.

Philip Lottmann, Sebastian Täubert, Dr. Saskia Villinger, Dr. David Ban, Dr. Donghan Lee, Dr. Pablo Trigo Mourino and Marta Carneiro: Thank you for all the useful discussions of all kind.

Thanks to all members of the $Z$-round and the whole department for the nice and friendly atmosphere. 
I'd like to especially thank Saskia, Philip and Ökki for the great friendship, the time I spent with each of you and all the things we've done and experienced.

Ein Riesendank geht an meine Eltern, Großeltern und Schwester, die mich nach anfänglichem Zögern doch immer auf meinem zweiten Bildungsweg restlos unterstützt haben. 


\section{Summary}

There are three distinct effects in nuclear magnetic resonance spectroscopy (NMR) that differ between paramagnetic and diamagnetic molecules in isotropic solution. These are residual dipolar coupling (RDC), pseudocontact shift (PCS) and paramagnetic relaxation enhancement (PRE). All of these effects are dependent on intermolecular angles and distances for a given nucleus of interest and can provide information about the structure and dynamics of a molecule. In order to obtain this valuable information, the molecule is required to display paramagnetic characteristics. One of the up-to-date methods achieves this requirement via the use of small molecular tags that coordinate paramagnetic metal ions. Most of these tags are attached to a protein via a disulfide bridge formed with a solvent exposed cysteine residue. Thus, in order to use this technique for DNA, new tagging strategies are required.

In this work, a modified nucleobase was synthesized allowing introduction of a sulfur moiety into the DNA. This provides a method to attach any tag that is based on a disulfide bridge, directly to the

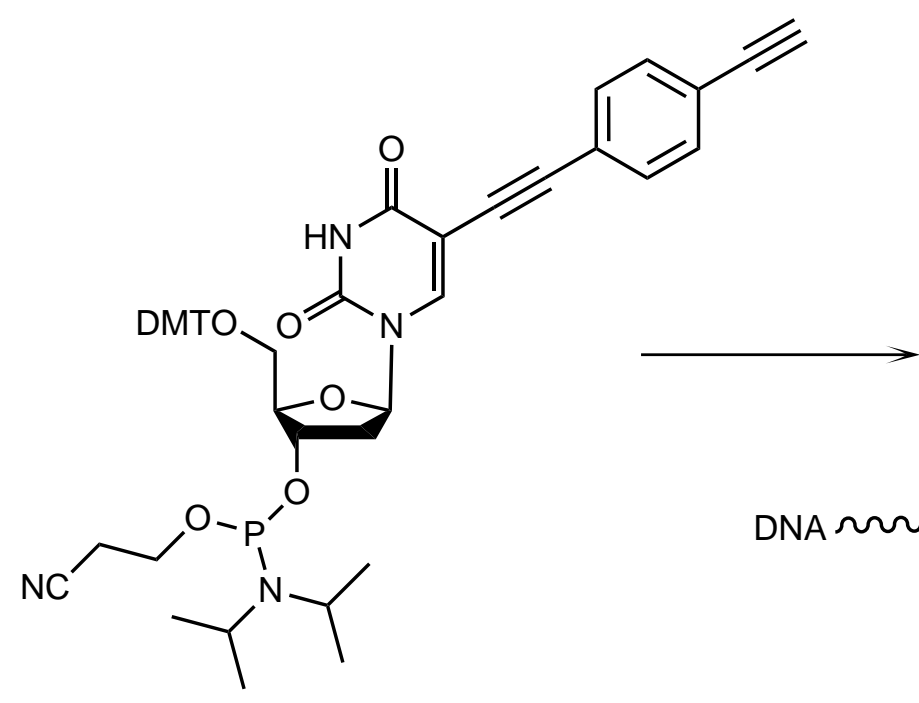

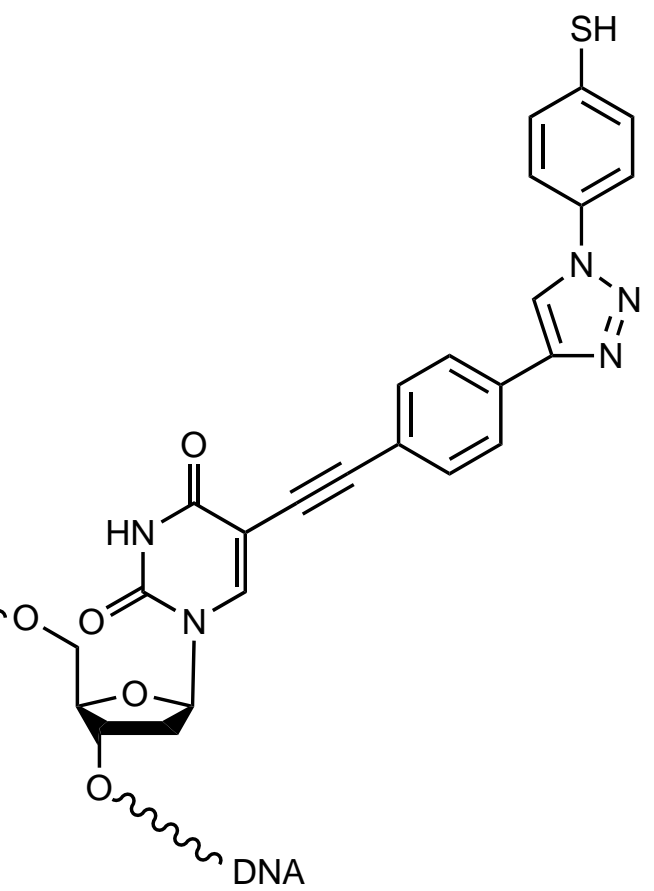

Left: The modified nucleobase containing a carbon triple bond. Right: The final modification of the DNA providing a sulfur moiety. 
DNA. With this nucleobase a carbon triple bond is introduced into the DNA strand, and a subsequent cycloaddition reaction leads to the free sulfur moiety.

The modified nucleobase was successfully tested by tagging a self-complementary DNA strand (24 nucleotides), in which the modified nucleobase was introduced during the DNA synthesis. The Cys-Ph-TAHA tag, preloaded with lutetium, terbium or thulium, was attached via a disulfide bond resulting in a tagged DNA strand loaded with a lanthanide ion. However, even with this milestone, a major aspect of this work was to develop a reliable and reproducible purification and sample preparation protocol. This became a critical element, since the tagging of DNA as compared to proteins is challenging by the ability for the phosphate backbone to coordinate lanthanide ions.

In the theoretical framework section, a complete step-by-step derivation of the three major paramagnetic effects starting from first principles is given . For the derivation of the equations describing the RDCs, PCSs and PREs, expressions for the dipolar Hamiltonians, cross relaxation rates, alignment induced RDCs, correlation functions and spectral densities are presented.

The second topic of this work is based on a different paramagnetic effect. In order to overcome the lower sensitivity of NMR compared to other spectroscopic methods, there are many reports on approaches that increase the polarization of the investigated nuclei, i.e. that create hyperpolarized species. One of these methods, photochemically induced dynamic nuclear polarization (photo CIDNP), is based on short lived radicals created by direct illumination of the sample in the magnet with a laser beam. Within the scope of this thesis, a photo CIDNP setup was planned, built and tested. The first experiments and results with triethylenediamine, tyrosine and 3-fluoro-L-tyrosine demonstrated the usefulness as well as the limitations of this technique. For 3-fluoro-L-tyrosine a complete analysis of the relaxation behaviour, including cross relaxation and cross-correlated relaxation, is given. 


\section{Zusammenfassung}

In der Kernspinresonanzspektroskopie (NMR) treten drei Effekte auf, die paramagnetische und diamagnetische Moleküle in isotroper Lösung unterscheiden: residuale dipolare Kopplung (RDC), Pseudokontaktverschiebung (PCS) und paramagnetische Relaxationsverstärkung (PRE). Alle drei Effekte sind abhängig von intermolekularen Winkeln und Abständen und können daher Informationen über die Struktur und Dynamik des Moleküls liefern. Um diese Informationen zu erhalten, muss das Molekül paramagnetische Eigenschaften aufweisen. Eine der heutzutage gebräuchlichen Methoden verwendet kleine molekulare Tags, die paramagnetische Metallionen koordinieren. Die meisten dieser Tags binden über eine Disulfidbrücke an Cysteine an der Proteinoberfläche. Um diese Methode für DNA anzuwenden werden daher neue Taggingstrategien benötigt.

Im Rahmen dieser Arbeit wurde eine modifizierte Nukleobase synthetisiert, mit der ein Schwefelatom in die DNA eingebracht werden kann. Diese Methode erlaubt es, jeden Tag an die DNA zu binden, der als Verbindungsmethode eine Disulfidbrücke nutzt. Mit der Nukleobase wird eine
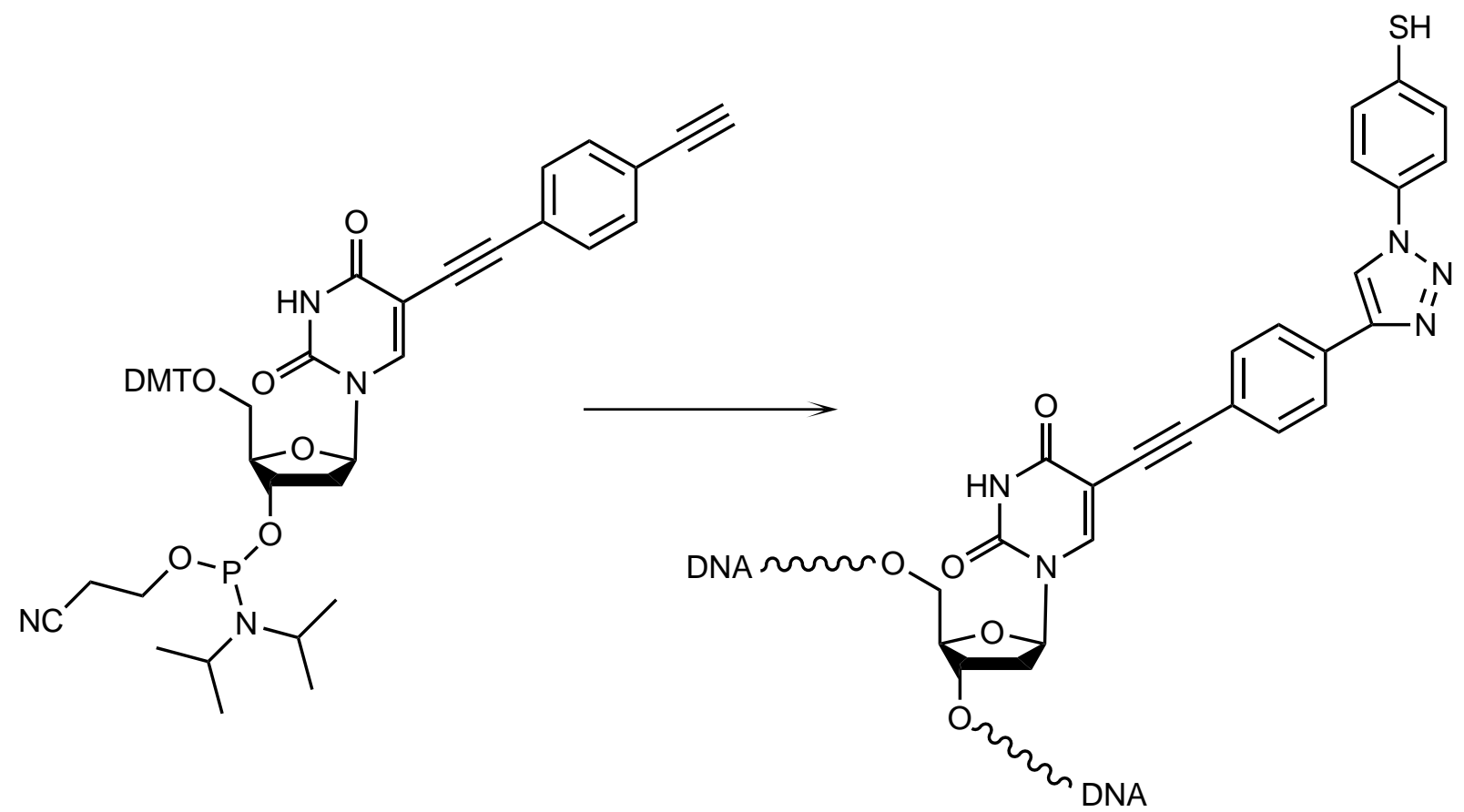

Links: Die modifizierte Nukleobase mit der Kohlenstoff-Dreifachbindung. Rechts: Die fertige Modifizierung der DNA mit der freien Thiolgruppe. 
Kohlenstoff-Dreifachbindung in die DNA eingefügt und mit Hilfe einer dipolaren Cycloaddition wird die freie Thiolgruppe eingebracht. Die modifizierte Nukleobase wurde erfolgreich an einem selbstkomplementären DNA-Strang (24 Nukleobasen) getestet. Die Nukleobase wurde während der Synthese der DNA eingefügt und der mit Lutetium, Terbium oder Thulium vorbeladene Cys-Ph-TAHA Tag wurde über eine Disulfidbrücke an die DNA gebunden. Die Beladung des Tags und die Taggingreaktion verliefen hierbei quantitativ. Nach diesem Erfolg war es ein Hauptaspekt dieser Arbeit, eine verlässliche und reproduzierbare Aufreinigungs- und Probenvorbereitungsmethode zu entwickeln. Diesem Punkt kommt besondere Bedeutung zu, da das Phosphatrückgrat der DNA, im Gegensatz zu Proteinen, Metallionen koordinieren kann.

Im Theorieteil dieser Arbeit ist eine komplette Herleitung der drei Hauptmerkmale paramagnetischer NMR gegeben. Diese Herleitung beginnt bei Grundbegriffen des Magnetismus und neben den Gleichungen für RDCs, PCSs und PREs werden Ausdrücke für den dipolaren Hamiltonoperator, Kreuzrelaxationsraten, kreuzkorrelierte Relaxationsraten, durch Alignment induzierte RDCs, Korrelationsfunktionen und spektrale Dichten gegeben.

Das zweite Thema dieser Arbeit basiert auf einem weiteren paramagnetischen Effekt. Um der reduzierten Empfindlichkeit der Kernspinresonanzspektroskopie verglichen mit anderen Spektroskopiemethoden entgegenzuwirken, wurden viele Methoden entwickelt, die auf eine Erhöhung der Polarisierung der Atomkerne zielen, d.h. um sogenannte hyperpolarisierte Kerne zu erzeugen. Eine dieser Methoden, die photochemisch erzeugte dynamische Kernpolarisierung (photo CIDNP), basiert auf kurzlebigen Radikalen, die durch direkte Laserbestrahlung der Probe im Magneten erzeugt werden. Im Rahmen dieser Arbeit wurde ein photo CIDNP Aufbau entworfen, gebaut und getestet. Die ersten Experimente und Resultate mit Triethylendiamin, L-Tyrosin und 3-Fluor-L-tyrosin zeigen die Vorteile und Grenzen dieser Methode auf. Für 3-Fluor-L-tyrosin wurde eine komplette Analyse des Relaxationsverhaltens, einschließlich der Kreuzrelaxation und der kreuzkorrelierten Relaxation, durchgeführt. 


\section{Contents}

\begin{tabular}{ll|l} 
Acknowledgments & VII
\end{tabular}

Summary - English

\begin{tabular}{lll} 
Summary - German & XI \\
\hline
\end{tabular}

1 Introduction 1

1.1 Paramagnetic Tagging Of Biomacromolecules . . . . . . . . . . . . . . 1

1.2 Photochemically Induced Dynamic Nuclear Polarization . . . . . . . . . . . . 11

1.3 The Aim Of This Work . . . . . . . . . . . . . . . . 13

2 Theoretical Framework $\quad 15$

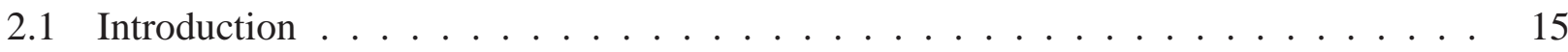

2.2 The Dipolar Hamiltonian $\ldots \ldots \ldots \ldots \ldots \ldots$

2.2.1 The Magnetic Field Of One Magnetic Moment . . . . . . . . . . . . 17

2.2.2 The Dipole-Dipole Interaction Hamiltonian . . . . . . . . . . . . . 17

2.3 Residual Dipolar Coupling - RDC . . . . . . . . . . . . . . 18

2.3.1 Dipolar Coupling Between Two Nuclei With The Same Spin . . . . . . . . . 19

2.3.2 Anisotropic Magnetic Susceptibility And The Magnetic Field Dependency Of

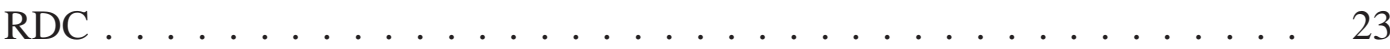

2.3.3 Sauper Order Matrix $\ldots \ldots \ldots \ldots \ldots \ldots$

2.4 Pseudocontact Shifts - PCS . . . . . . . . . . . . . . . . . . 27

2.4.1 Interaction Of Nucleus And The Paramagnetic Center . . . . . . . . . 28

2.4.2 Alignment Effects On The PCSs . . . . . . . . . . . . . . . . . 29

2.5 Residual Chemical Shift Anisotropy - RCSA . . . . . . . . . . . . . . 31

2.6 Paramagnetic Relaxation Enhancement - PRE . . . . . . . . . . . . . 32

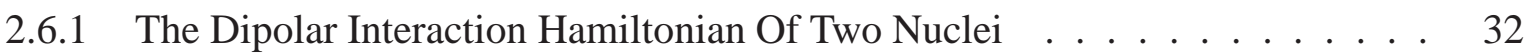

2.6.2 The Dipolar Interaction Hamiltonian Of A Nucleus And An Electron . . . . . 36

2.6.3 Lioville - Von Neumann Equation . . . . . . . . . . . . . . . 37

2.6 .4 The Master Equation $\ldots \ldots \ldots \ldots \ldots \ldots$

2.6.5 Relaxation Of Two Like Spins . . . . . . . . . . . . . . . . . . 41 
2.6.6 Relaxation Of Unlike Spins - Nucleus And Electron . . . . . . . . . . . 45

2.6.7 Relaxation Of Two Unlike Nuclei . . . . . . . . . . . . . . . 46

2.6.8 Calculation Of The Spectral Densities . . . . . . . . . . . . 47

2.6 .9 Curie Spin Relaxation . . . . . . . . . . . . . . . . . 50 50

2.7 Concluding Remarks . . . . . . . . . . . . . . . . . . 52

2.8 Photochemically Induced Dynamic Nuclear Polarization (Photo CIDNP) . . . . . . 53

2.8.1 The Radical Pair Mechanism . . . . . . . . . . . . . . . . . 53

2.8.2 Spin Sorting In A Radical Pair . . . . . . . . . . . . . . . 56 56

2.8 .3 Kaptein's Rules . . . . . . . . . . . . . . . . . 5 58

2.8.4 Cross-Correlated Relaxation And Cross Relaxation . . . . . . . . . . . . . 59

\section{Photo CIDNP}

3.1 The Experimental Setup . . . . . . . . . . . . . . . . . . 61

3.2 The Investigated Molecules $\ldots \ldots \ldots \ldots$

3.3 Results \& Analysis . . . . . . . . . . . . . . . . . . . . 66

3.3.1 Triethylenediamine ........................... 66

3.3.2 3-Fluorotyrosine ............................. 69

3.3 .3 Tyrosine .................................. 74

3.3 .4 Photobleaching ........................... 75

3.4 Discussion \& Outlook . . . . . . . . . . . . . . . . 76

3.4 .1 Concluding Remarks . . . . . . . . . . . . . . . . . 79

\begin{tabular}{ll|l|}
\hline & Tagging Strategy For DNA & 81 \\
\hline
\end{tabular}

4.1 Alteration Of The Linkage Type Of Cys-Ph-TAHA . . . . . . . . . . . . . 83

4.2 Introduction Of A Sulfur Moiety Into DNA . . . . . . . . . . . . . . 91

4.3 Shortening Of The Linker Of The Cys-Ph-TAHA Tag . . . . . . . . . . . . . 93

4.4 Results . . . . . . . . . . . . . . . . . . . . . . . . . . . . . . . . . . .

4.5 Discussion \& Outlook . . . . . . . . . . . . . . . . . . 103

4.5 .1 Concluding Remarks . . . . . . . . . . . . . . . . . . 105

5 Experimental Part $\quad 107$

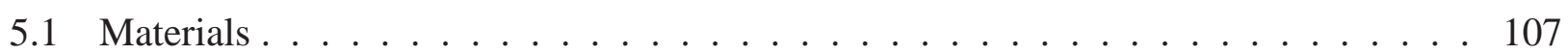




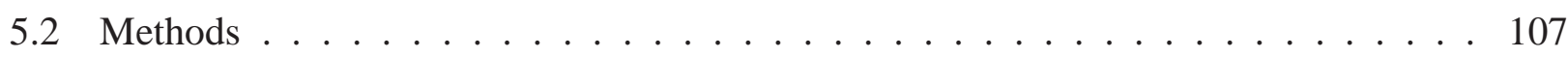

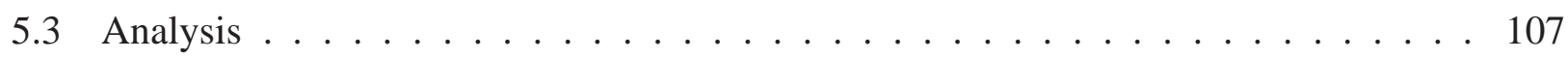

5.3.1 Nuclear Magnetic Resonance Spectroscopy . . . . . . . . . . . . . . . 107

5.3.2 HPLC Chromatography . . . . . . . . . . . . . . . 108

5.3 .3 Mass Spectrometry .......................... 108

5.3 .4 UV/Vis-Spectroscopy . . . . . . . . . . . . . . . . . 108

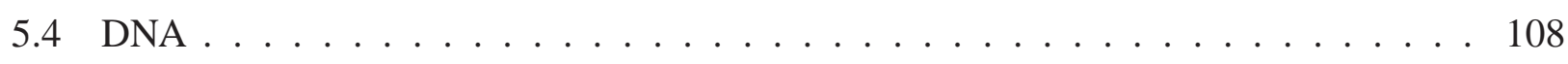

5.5 Experimental Details . . . . . . . . . . . . . . . . . . 109

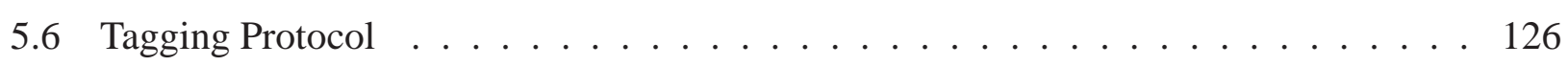

\begin{tabular}{l|l|} 
References & 127 \\
\hline
\end{tabular}

\begin{tabular}{l|l} 
List Of Abbreviations & 137 \\
\hline
\end{tabular}

List Of Figures

6 Appendix

\begin{tabular}{ll} 
Curriculum Vitae & 153 \\
\hline
\end{tabular} 



\section{Introduction}

\subsection{Paramagnetic Tagging Of Biomacromolecules}

Since its discovery in 1946, nuclear magnetic resonance spectroscopy (NMR) has become a well established and daily used technique [Purcel11946] [Bloch1946a] [Bloch1946b]. Structure determination of small molecules and biomacromolecules as well as investigation of function and interaction of molecules are areas in which NMR is used. However, most molecules are diamagnetic and therefore NMR techniques are focused on diamagnetic molecules. Paramagnetic molecules pose problems of line broadening and extinction of resonances close to the paramagnetic center (paramagnetic relaxation enhancement, PRE, review article: [Clore2009]). Nevertheless, paramagnetic molecules exhibit additional NMR observables that can be used for structure determination as explained in the following.

Solomon and Bloembergen derived equations for the paramagnetic relaxation enhancement, which show that this effect can yield valuable information about structural parameters in the form of distances between the nucleus and the paramagnetic center [Solomon1955] [Bloembergen1957a]. The same information can be obtained by pseudocontact shifts (PCS) which are observed as a change of the chemical shift of a nucleus due to the interaction between its magnetic moment and the anisotropic magnetic susceptibility of a paramagnetic center. This effect was first described by McConnell \& Robertson in 1958 [McConnell1958].

Anisotropic magnetic susceptibility has another influence on the observed NMR spectra in terms of changes in the coupling constants between two nuclei. In diamagnetic molecules in isotropic solution, only the scalar coupling $J$ is observed. The dipolar coupling $D$ is dependent on the direction of the internuclear vector relative to the external magnetic field, is averaged to zero and leads to line broadening dependening on the "speed" of Brownian motion in solution. In case of a preferred orientation of the molecule with respect to the external magnetic field (alignment), the dipolar coupling is not averaged to zero and residual dipolar couplings (RDC) can be observed. This partial alignment occurs due to the interaction between the anisotropic magnetic susceptibility and the external magnetic field. Residual dipolar couplings also contain information about distances and angles in the molecular frame. The first description of this effect in solution was given for small molecules by Bothner-By et 
al. with paramagnetic molecules containing cobalt [Bothner-By1981] and for proteins by Tolman et $a l$. with cyano metmyoglobin, which has a highly anisotropic magnetic susceptibility due to the iron ion [Tolman1995].

Although there are several excellent reviews and overview articles in the literature [Bertini2002] [John2007] [Otting2008] [Clore2009] [Nicholas2010] they use different notations such that interconnections between the various parameters are non-obvious. One aim of this work was therefore to provide a unified and complete description and a consistent derivation of the various effects of paramagnetic NMR.

Another way of inducing alignment in molecules, which results in measurable RDCs is to use external alignment media like liquid crystals [Bax1997] [Hansen1998] [Ottiger1998b] [Rueckert2000] [Thiele2003] or stretched and compressed gels [Tycko2000] [Sass2000] [Haberz2005] [Kobzar2005] [Kummerloewe2007] [Schmidt2012a].

Furthermore, even diamagnetic molecules can show a partial alignment if their magnetic susceptibility is anisotropic. This was first shown for small molecules by Gayathri et al., for DNA strands by Kung et al. and for proteins by Tjandra et al. [Gayathri1982] [Kung1995] [Tjandra1996a].

To obtain this valuable information, scientists turned diamagnetic molecules paramagnetic. This was achieved either by increasing the affinity of metal binding proteins to paramagnetic ions (for example Bertini et al. achieved a selective lanthanide binding site in calmodulin by selective mutation [Bertini2003]) or by attaching paramagnetic domains to the molecule, for example a zinc finger moiety [Gaponenko2000] or a lanthanide binding domain [Woehnert2003]. To avoid an extensive increase in the molecular weight of the target molecules, small paramagnetic tags have been developed, which can be attached to molecules. These tags are mostly preloaded with lanthanides due to the metal's highly anisotropic magnetic susceptibility [Otting2008]. The advantage of this internal alignment is the oppertunity to observe domain motions and dynamics if the alignment is induced by just one domain [Bertini2004] [Rodriguez2006] [Zhang2007]. The distinction between different homodimers in the case where only one of them is specifically paramagnetically labeled is another reported advantage [Gaponenko2002].

The importance of this information can be shown by a short historical overview of the developed tags. The first alignment of a protein induced by a small molecule tag was reported 2002. There, $S$ - 
(2-pyridylthio)-cysteaminyl ethylenediamine tetraacetic acid [Dvoretsky2002], which binds via one disulfide bridge to a cysteine, was utilized (Fig:1.1). This tag, however, has a pseudo-asymmetric nitrogen center which forms diastereomers upon chelating a metal ion and consequently shows a dublicated signal set in the NMR spectra [Ikegami2004].<smiles>O=C(O)CN(CCN(CC(=O)O)CC(=O)NCCSSc1ccccn1)CC(=O)O</smiles>

Figure 1.1: $S$-(2-Pyridylthio)cysteaminyl ethylenediamine tetraacetic acid.

Woehnert et al. described in 2003 the alignment of ubiquitin by a lanthanide binding amino acid sequence attached to the protein's N-terminus [Woehnert2003].

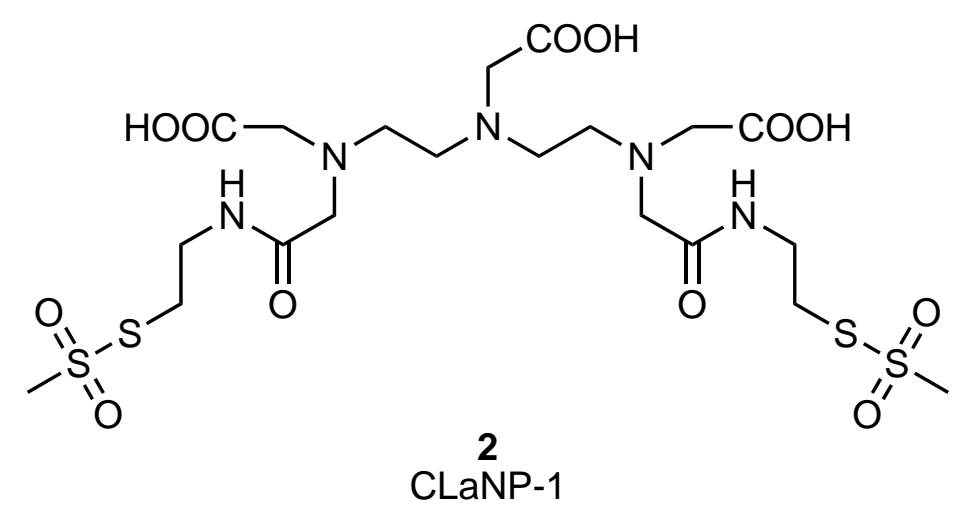

Figure 1.2: Structure of CLaNP-1.

In 2004, Prudêncio et al. described a new tag based on diethylene triaminepentaacetic acid (DTPA) named CLaNP-1 (caged lanthanide NMR probe), which binds via two disulfide bridges to two cysteine residues of the target protein (pseudoazurin) (Fig:1.2) [Prudencio2004]. The disadvantage of this tag lies in the formation of five different isomers, leading to five different sets of signals in the NMR spectra, which is highly unfavorable [Franklin1994]. 
The following second generation of EDTA based tags had no pseudo-asymmetric centers and could be synthesized enantiomerically pure (Fig:1.3) [Ikegami2004] [Leonov2005]. The binding motif of these two tags is a single disulfide bridge to a cysteine. The influence of the additional information on structure calculation was demonstrated by Haberz et al. \& Rodriguez-Castañeda et al. for trigger factor [Haberz2006] [Rodriguez2006].<smiles>CS(=O)(=O)SC[C@H](NC(=O)[C@@H](CN(CC(=O)O)CC(=O)O)N(CC(=O)O)CC(=O)O)C(=O)O</smiles>

3a $(R, R)$<smiles>CS(=O)(=O)SC[C@H](NC(=O)[C@@H](CN(CC(=O)O)CC(=O)O)N(CC(=O)O)CC(=O)O)C(=O)O</smiles><smiles>CS(=O)(=O)Sc1ccc(NC(=O)C(CN(CC(=O)O)CC(=O)O)N(CC(=O)O)CC(=O)O)cc1</smiles>

4a $(R)$<smiles>CS(=O)(=O)Sc1ccc(NC(=O)[C@@H](CN(CC(=O)O)CC(=O)O)N(CC(=O)O)CC(=O)O)cc1</smiles>

$\mathbf{4 b}(S)$

Figure 1.3: Structures of the two enantiomers of the second generation EDTA based tags.

In 2007, two new tags based on 1,4,7,10-tetraazacyclododecane-1,4,7,10-tetraacetic acid (DOTA) were published (Fig:1.4] [Vlasie2007] [Keizers2007]. CLaNP-3 was tested with pseudoazurin. The tag binds via two disulfide bridges to two cysteine residues and induces two sets of NMR signals due to helical chirality. The tag forms two enantiomeric pairs of diastereomers and two of these four isomers are populated [Vlasie2007]. CLaNP-5 was tested with pseudoazurin in two different modifications, of which one had the double binding motif to two cysteine residues of the protein and one was connected via a single disulfide bridge. In contrast to CLaNP-3, the higher sterical requirement of CLaNP-5 causes the population of just one of the four isomers [Keizers2008]. With 
CLaNP-5.1, RDCs up to $6 \mathrm{~Hz}$ were measured whereas the RDCs for CLaNP-5.2 were significantly smaller. In 2011, Dasgupta et al. reported about the use of CLaNP-5.1 to tag the C-terminal domain of the calmodulin mutation mentioned above, in which the paramagnetic center is at the $\mathrm{N}$-terminus [Dasgupta2011]. The observed RDCs and PCSs were used to investigate the conformational space of calmodulin.

The main disadvantage of CLaNP-5.2 and all other tags which binds via two disulfide bridges is the necessity of a priori knowledge about the protein. Either the existence of two cysteine residues in close proximity has to be known or a suitable mutation/double-mutation has to be prepared.

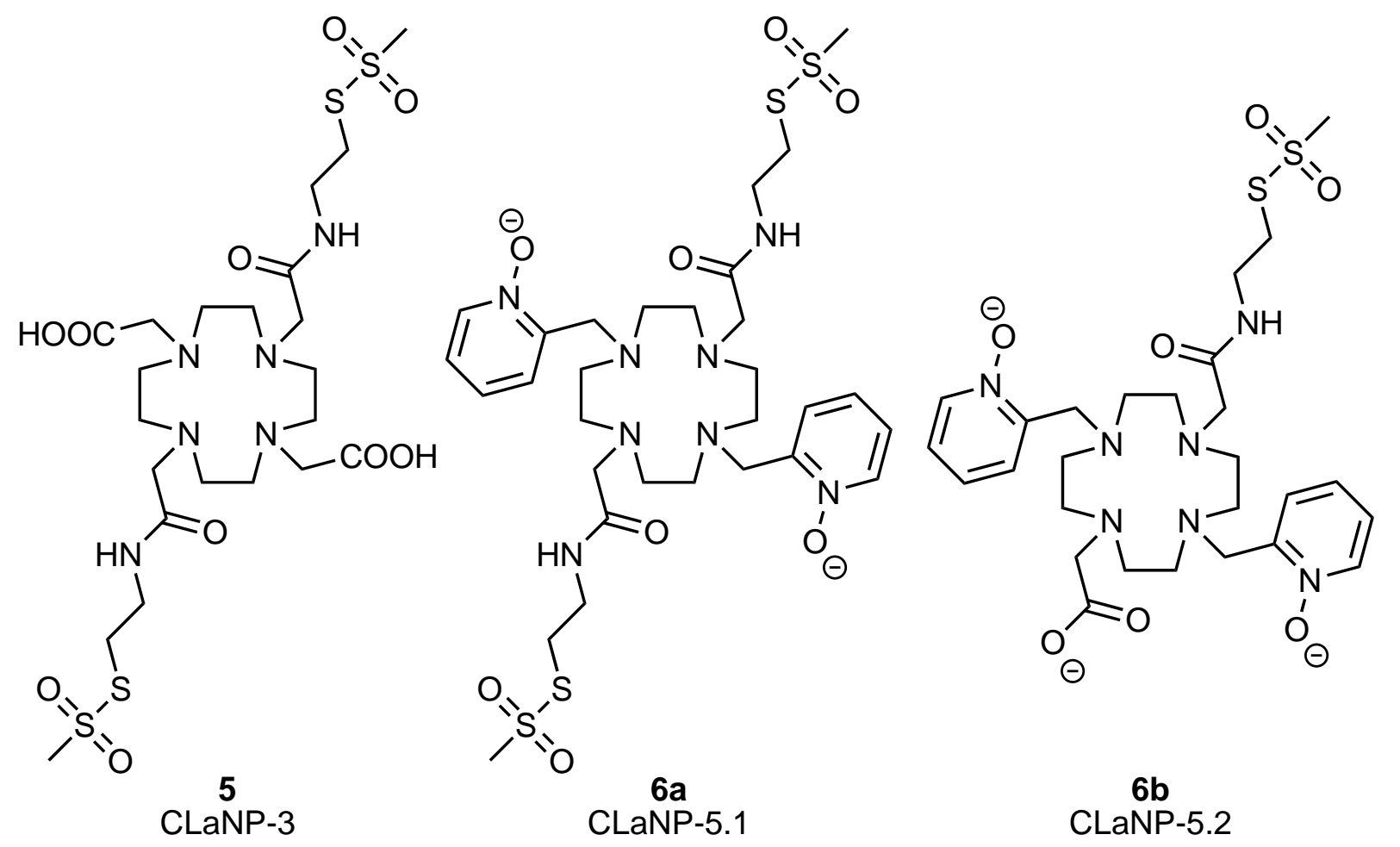

Figure 1.4: Structures of CLaNP-3, CLaNP-5.1 and CLaNP-5.2.

Based on the DOTA tags and previous work about multiple methylated DOTA derivatives [Ranganathan2002a] [Ranganathan2002b], Haeussinger et al. developed an eightfold methylated DOTA derivative, which was first tested on ubiquitin [Haeussinger2009]. The tag binds via a single disulfide bridge and the rigid backbone of the tag allows for the population of just one isomer (Fig:1.5). cis-trans isomerization of the linker's peptide bond leads to a second signal set with ap- 


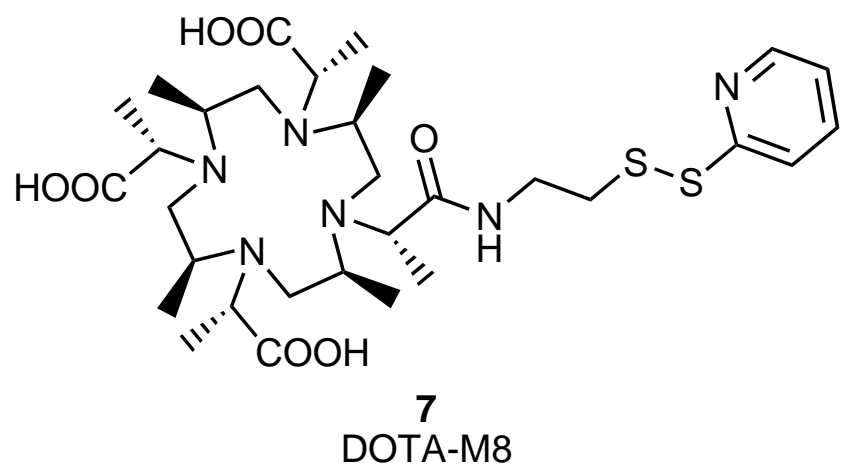

Figure 1.5: Structure of the DOTA-M8 tag.

proximately 15-20\% intensity [Haeussinger2009].

Three more rigid DOTA based tags are described in the literature. Graham et al. measured PCS and RDC with 2,2',2"'-(10-(2-Oxo-2-(2-(pyridin-2-yldisulfanyl)ethylamino)-ethyl)-1,4,7,10-tetraazacyclododecane-1,4,7-triyl)tris(N-((S)-1-phenylethyl)acetamide) (8 C1 Fig:1.6) tagged to the N-terminal domain of the arginine repressor and an A28C mutant of ubiquitin [Graham2011].

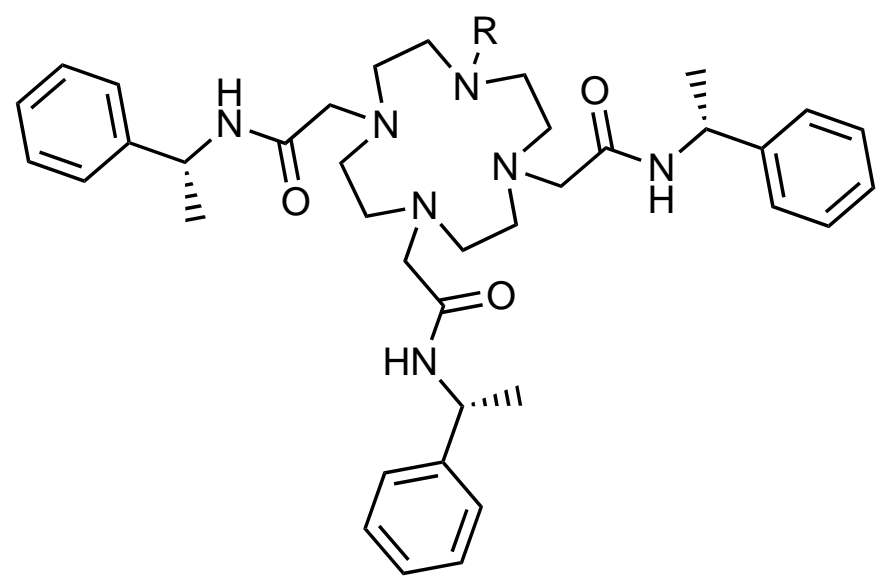

8<smiles>O=C(C=PCl)NCSSc1ccccn1</smiles>

9 C3 $\mathrm{R}=$<smiles>C#CCNC(=O)P=[18O]</smiles>

Figure 1.6: Structures of the C1, C3 and C4 tag.

This tag binds via a single disulfide bridge to a cysteine residue and only one isomer is populated so that the tag yields just one set of signals for the paramagnetic species. Two modifications to $\mathrm{C} 1$ were developed by Loh et al. (C3 \& C4 Fig,1.6), for which the binding motif changed to a 1,3-dipolar cycloaddition of the tag's carbon triple bond and an azido-phenylalanine of the protein [Loh2013]. 
Another approach to very small tags is based on dipicolinic acid (DPA) (Fig:1.7). These three dentate tags are bound via a single disulfide bridge to a cysteine residue and utilize carboxyl groups of neighbouring amino acids to stabilize the metal ion. The first of these tags was described in 2008 (4MMDPA (4-mercaptomethyl-dipicolinic acid) [Su2008]) and it's linker was flexible due to the methylene group between the thiole and the dipicolinic acid, whereas this group was removed in both, 3MDPA (3-mercapto-dipicolinic acid) [Man2010] and 4MDPA (4-mercapto-dipicolinic acid) [Jia2011a] (Fig:1.7).

The complex of a paramagnetic lanthanide ion $(\mathrm{Ln})$ with three DPA $\left(\left[\operatorname{Ln}(\mathrm{DPA})_{3}\right]^{3-}\right)$ binds site specifically non-covalently to a protein and induces PRE and PCS [Yagi2010]. The complex binds preferable to positively charged amino acids which can be introduced into the protein to create a binding site [Jia2011b]. Recently, different substitution patterns of DPA were investigated by Wei $e t$ al. [Wei2013].<smiles>O=C(O)c1cccc(C(=O)O)n1</smiles><smiles>O=C(O)c1ccc(S)c(C(=O)O)n1</smiles>

13

3MDPA<smiles>O=C(O)c1cc(CS)cc(C(=O)O)n1</smiles>

12

4MMDPA<smiles>O=C(O)c1cc(S)cc(C(=O)O)n1</smiles>

14

4MDPA

Figure 1.7: Structures of DPA, 4MMDPA, 3MDPA and 4MDPA.

Similar to the small DPA based tags, Swarbrick et al. developed a small tag based on iminodiacetic acid (Fig:1.8) [Swarbrick2011]. This tag was tested on ubiquitin, binds via a cystein residue and needs at least one other carboxyl group next to the binding cysteine to stabilize the complex. 


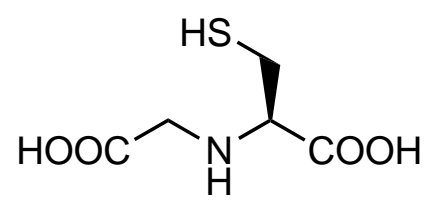

15

Figure 1.8: Structure of the iminodiacetic acid based tag.

The Cys-Ph-TAHA tag (cysteine-phenyl-triaminohexaacetic acid) (Fig:[1.9], developed in our group by Peters et al., was successfully tested on ubiquitin and a ternary complex of lac repressor, DNA and inducer (by Boelens / Utrecht University) [Peters2011]. Due to the lack of stereocenters, the tag yields just one set of signals for the paramagnetic species and the observed PCSs and RDCs of up to 2 ppm and $8 \mathrm{~Hz}$, respectivally, are in excellent agreement with backcalculated values. Despite the observed paramagnetic effetcs the connection of the Cys-Ph-TAHA tag to the protein via the cystein linker of the tag induces a distinct flexibility of the tag which reduces the measurable PCSs and RDCs. A secondary aim of this work was therefore, to shorten the linker of the tag.

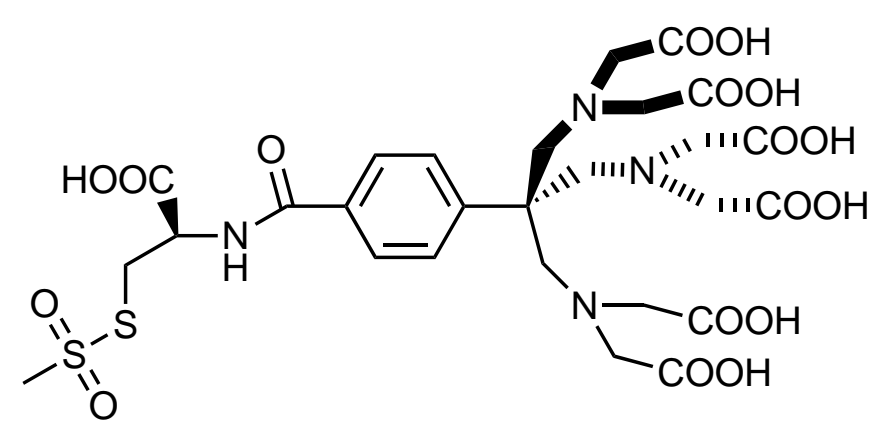

16

Figure 1.9: The Cys-Ph-TAHA tag (cysteine-phenyl-triaminohexaacetic acid).

The most recent version of the CLaNP tags was published in 2012 by Liu et al.. CLaNP-7 has a lower charge $(+1)$ than CLaNP-5 (+3), which reduces the change in the surface potential at the binding site and exhibits a different anisotropic magnetic susceptibility. Therefore, by using two samples with the two different tags, two sets of signals can be recorded for the paramagnetic species, which increases the accuracy of the obtained information [Liu2012]. The tag binds via two disulfide 


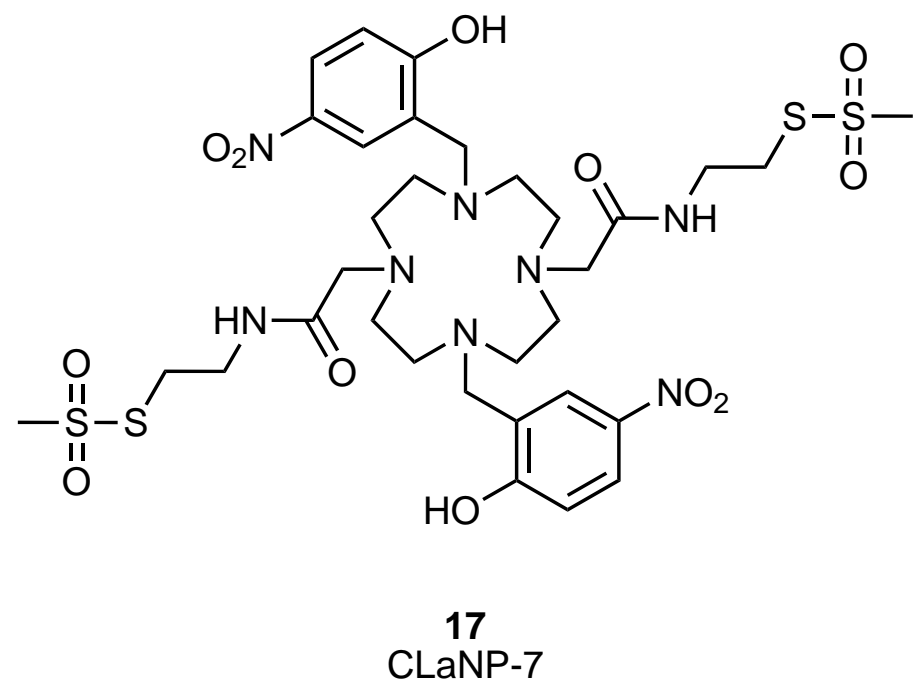

Figure 1.10: The caged lanthanide NMR probe, CLaNP-7.

bridges to two cysteine residues and was tested with pseudoazurin and cytochrom $c$.

Another binding motif of DPA based tags to proteins was presented by Li et al.. The 4VDPA (4vinyl-dipicolinic acid) tag (Fig:1.11) binds via a thiol-ene reaction specifically to a cysteine residue and was tested on ubiquitin and arginine repressor [Li2012]. The same binding motif is used by the 4-vinyl(pyridine-2,6-diyl)bis-methylenenitrilo tetrakis(acetic acid) tag (4VPyMTA), which was tested on ubiquitin (Fig:1.11] [Yang2013]. Similar to other DPA based tags, a carboxyl group of a neighbouring amino acid is necessary to stabilize the complex.

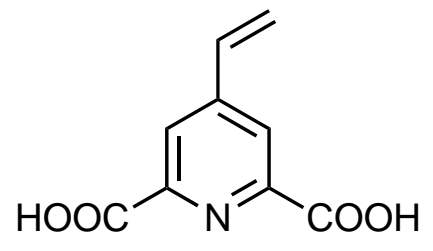

18 4VDNP<smiles>C=Cc1cc(CN(CC(=O)O)CC(=O)O)nc(CN(CC(=O)O)CC(=O)O)c1</smiles>

19

4VPyMTA

Figure 1.11: Structures of the two thiol-ene binding tags 4VDPA and 4VPyMTA.

So far, the only molecules investigated using the concept of paramagnetic tagging are proteins. Except for the $\mathrm{C} 3(\mathbf{9})$ and the $\mathrm{C} 4$ tag (10), the only binding motifs used are solvent exposed cysteine 
residues. In the case that the needed cysteine residues are absent, mutations of the proteins can be prepared, in which the cysteine is introduced at the desired position. For proteins, the tagging methods are well established, whereas for other biomacromolecules no such methods are known.

DNA and RNA are important biomacromolecules and the oppertunity to obtain the additional information provided by paramagnetic tagging would be equally useful. One aim of this work was the development of a method for tagging of DNA molecules. For this topic, the Cys-Ph-TAHA tag, recently developed in our group, was used. A short DNA strand served as a test molecule for the tagging procedure. DNA and RNA consist of the two purine bases adenine and guanine and the three pyrimidine bases uracil (only RNA), thymine (only DNA) and cytosine (Fig:1.12). All bases are bound to ribose (RNA) or 2-deoxyribose (DNA) and the sugars are connected via the phosphate backbone. Contrary to proteins, there exists no naturally occuring sulfur moiety in nucleic acids.

Purine bases<smiles>Nc1ncnc2[nH]cnc12</smiles>

20 Adenine<smiles>Nc1nc2[nH]cnc2c(=O)[nH]1</smiles>

21

Guanine<smiles>O=c1cc[nH]c(=O)[nH]1</smiles>

22 Uracil

Pyrimidine bases<smiles>Cc1c[nH]c(=O)[nH]c1=O</smiles>

23

Thymine<smiles>Nc1cc[nH]c(=O)n1</smiles>

Cytosine

Figure 1.12: Structures of the five nucleobases. 


\subsection{Photochemically Induced Dynamic Nuclear Polarization}

Another form of paramagnetic NMR was first described in the literature in 1967. In the first experiments, non equilibrium polarization of nuclei generated during reactions of organometallic compounds, peroxides or azo compounds in the spectrometer was observed. This polarization led to increased absorptive or emmisive NMR signals [Bargon1967] [Ward1967]. It was first believed that this effect has a similar origin to that of dynamic nuclear polarization, which occurs due to cross relaxation of electrons and nuclei [Hausser1968], and therefore this effect was named chemically induced dynamic nuclear polarization. In the following years, the theoretical aspects of this technique were investigated more closely. Based on short living radicals, the dominant mechanism was found to be the radical pair mechanism (or radical pair theory), which will be explained in detail in the next section [Closs1969a] [Kaptein1969] [Adrian1970] [Closs1970] [Kaptein1971] [Pedersen1973a] [Pedersen1973b] [Pedersen1974] [Adrian1977a]. Under special circumstances, another process called triplet mechanism can take place [Atkins1977] [Adrian1977b] [Hore1979b].

The described effect was also observed during photochemical reactions of diphenylazomethane for which the sample was illuminated with a high power light source. This phenomenon is called photochemically induced dynamic nuclear polarization (photo CIDNP) [Closs1969b]. An overlay of NMR spectra displaying this effect is shown in Fig:1.13.

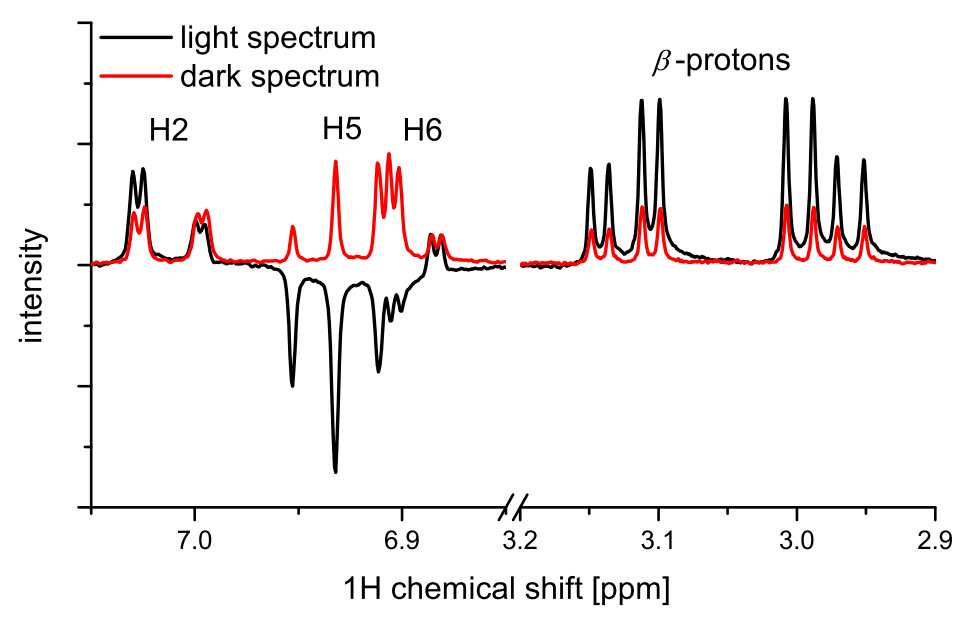

Figure 1.13: Demonstration of the photo CIDNP effect for 3-fluoro-L-tyrosine. 
The possible application of this technique to biomolecules was investigated by Kaptein et al. by using flavins to enhance the NMR signals of tyrosine [Kaptein1978]. Following this early work, surface investigations of proteins were accomplished in which flavins were used to enhance the NMR signals of solvent exposed amino acids [Hore1993].

The possibility to use the increased polarization produced in the photo CIDNP experiments to subsequently increase the polarization of other nuclei via cross relaxation was first discussed by Bargon \& Gardini [Bargon1979]. Since then, the feasibility to use photo CIDNP as a general enhancement technique for NMR was not well explored until in 2004 Kuprov et al. described cross relaxation in 3-fluoro-L-tyrosine [Kuprov2004a]. The practical aspects of multi flash experiments in time resolved photo CIDNP experiments were explored by Goez et al., whereas the use of photo CIDNP in two dimensional heteronuclear NMR experiments was described by Sekhar \& Cavagnero [Goez2005] [Sekhar2009].

The last aim of this work was to plan and build a photo CIDNP setup and to further investigate the possible benefits of this technique. 


\subsection{The Aim Of This Work}

This work can be split into four different parts with their respective aims:

1. The complete step by step derivation of the three major effects of paramagnetic tagging of molecules:

- paramagnetic relaxation enhancement

- pseudocontact shift

- residual dipolar coupling.

2. Developement of a convenient tagging strategy for DNA/RNA molecules.

3. Shorten the linker of the Cys-Ph-TAHA tag.

4. Planing, building and investigating the possible benefits of a photo CIDNP setup. 



\section{Theoretical Framework}

\subsection{Introduction}

The aim of this section is to provide the reader with a complete step-by-step derivation of the most important paramagnetic NMR effects. Focusing on NMR in isotropic solution, these parameters are residual dipolar couplings (RDC, Eq:11), pseudocontact shifts (PCS, Eq:2), paramagnetic relaxation enhancement (PRE, Eq:3 \& Eq:44) and cross-correlated relaxations including the dipolar coupling between the observed spin and the paramagnetic center (dipole-dipole-Curie-spin cross-correlated relaxation, $\mathrm{Eq}: 5 \mathrm{Eq}: 6$. In addition, there is the dynamic frequency shift associated with all the relaxation processes, derived from the imaginary part of the spectral density function (Eq:7).

$$
\overline{\mathrm{D}_{k l}^{\mathrm{RDC}}}=-\frac{1}{8 \pi^{2}} \frac{\gamma_{k} \gamma_{l} \hbar}{r_{k l}^{3}} \frac{B_{0}^{2}}{15 k T}\left(\left(3 \cos ^{2}[\theta]-1\right)\left(\chi_{z z}-\frac{\chi_{x x}+\chi_{y y}}{2}\right)+\frac{3}{2}\left(\chi_{x x}-\chi_{y y}\right) \cos [2 \varphi] \sin ^{2}[\theta]\right)
$$

$$
\begin{gathered}
\delta_{\mathrm{PCS}}=\frac{1}{12 \pi r_{I S}^{3}}\left(\left(3 \cos ^{2}[\theta]-1\right)\left(\chi_{z z}-\frac{\chi_{x x}+\chi_{y y}}{2}\right)+\frac{3}{2}\left(\chi_{x x}-\chi_{y y}\right) \cos [2 \varphi] \sin ^{2}[\theta]\right) 10^{6} \\
\frac{1}{T_{1}}=\frac{2}{5}\left(\frac{\mu_{0}}{4 \pi}\right)^{2} \frac{\gamma_{I}^{2} g_{e}^{2} \mu_{B}^{2}}{r_{I S}^{6}} S(S+1)\left[\frac{\tau_{c}}{1+\tau_{c}^{2} \omega_{I}^{2}}\right] \\
\frac{1}{T_{2}}=\frac{1}{15}\left(\frac{\mu_{0}}{4 \pi}\right)^{2} \frac{\gamma_{I}^{2} g_{e}^{2} \mu_{B}^{2}}{r_{I S}^{6}} S(S+1)\left[4 \tau_{c}+\frac{3 \tau_{c}}{1+\tau_{c}^{2} \omega_{I}^{2}}\right] \\
\frac{1}{T_{1}}=\frac{6}{5}\left(\frac{\mu_{0}}{4 \pi}\right)^{2} \frac{\gamma_{I}^{2} g_{e}^{4} \mu_{B}^{4} B_{0}^{2}}{(3 k T)^{2} r_{I S}^{6}}[S(S+1)]^{2}\left[\frac{\tau_{r}}{1+\tau_{r}^{2} \omega_{I}^{2}}-\frac{\tau_{c}}{1+\tau_{c}^{2} \omega_{I}^{2}}\right]
\end{gathered}
$$




$$
\begin{aligned}
& \frac{1}{T_{2}}=\frac{1}{5}\left(\frac{\mu_{0}}{4 \pi}\right)^{2} \frac{\gamma_{I}^{2} g_{e}^{4} \mu_{B}^{4} B_{0}^{2}}{(3 k T)^{2} r_{I S}^{6}}[S(S+1)]^{2}\left[4 \tau_{r}+\frac{3 \tau_{r}}{1+\tau_{r}^{2} \omega_{I}^{2}}-4 \tau_{c}-\frac{3 \tau_{c}}{1+\tau_{c}^{2} \omega_{I}^{2}}\right] \\
& \int_{0}^{\infty} \overline{F^{(q)}(t) F^{(q)^{\star}}(t+\tau)} \exp \left[-i \omega_{(p)}^{(q)} \tau\right] \mathrm{d} \tau \\
& =\operatorname{Re} \int_{0}^{\infty} \overline{F^{(q)}(t) F^{(q)^{\star}}(t+\tau)} \exp \left[-i \omega_{(p)}^{(q)} \tau\right] \mathrm{d} \tau+\operatorname{Im} \int_{0}^{\infty} \overline{F^{(q)}(t) F^{(q)}{ }^{\star}(t+\tau)} \exp \left[-i \omega_{(p)}^{(q)} \tau\right] \mathrm{d} \tau \\
& =J_{(p)}^{(q)}\left(\omega_{(p)}^{(q)}\right)+i K_{(p)}^{(q)}\left(\omega_{(p)}^{(q)}\right)
\end{aligned}
$$

As obvious from the formulae, the paramagnetic effects report on various angles with respect to the susceptibility tensor of the paramagnetic center and distances, mostly with respect to the paramagnetic center. During the derivation of the equations for the paramagnetic effects, other important effects are described, namely relaxation and alignment induced residual dipolar couplings which are not directly connected to paramagnetic NMR but are important for the derivation of the equations for the paramagnetic effects.

In the second part of this section, the fundamental basics of another advantageous form of paramagnetic NMR (photochemically induced dynamic nuclear polarization, photo CIDNP) are derived. This technique relies on short living paramagnetic species formed by laser irridiation of the sample in the NMR spectrometer which increase the magnetization of the nuclei. Subsequent dipolar cross relaxation can transfer the magnetization to other nuclei (Eq:8).

$$
\sigma_{\mathrm{IS}}=\frac{1}{10}\left(\frac{\mu_{0}}{4 \pi}\right)^{2} \frac{\gamma_{\mathrm{I}}^{2} \gamma_{\mathrm{S}}^{2} \hbar^{2}}{r_{\mathrm{IS}}^{6}}\left[\frac{6 \tau_{c}}{1+\tau_{c}^{2}\left(\omega_{\mathrm{S}}+\omega_{\mathrm{I}}\right)^{2}}-\frac{\tau_{c}}{1+\tau_{c}^{2}\left(\omega_{\mathrm{S}}-\omega_{\mathrm{I}}\right)^{2}}\right]
$$

\subsection{The Dipolar Hamiltonian}

All paramagnetic effects of a molecule with isotropic rotational diffusion on a sub-microsecond time scale, described in the next chapters, involve the interaction between magnetic dipoles. The residual dipolar coupling arises from this interaction between dipoles and can be observed because of the alignment induced by the paramagnetic center. Pseudocontact shifts and paramagnetic relaxation 
enhancement have their origin in the dipolar interaction between a paramagnetic center and the nucleus. For these effects, the starting expression for calculations is the dipolar interaction Hamiltonian which will be introduced first. The calculations in this section are according to Principles of Nuclear Magnetism [Abragam1961] and The Feynman Lectures on Physics - Book 2 Electromagnetism and Matter [Feynman1964]. For simplicity the calculations are reduced to isotropic solutions, electrons, nuclei with spin $1 / 2$ and the paramagnetic center is assumed to be a point dipole.

\subsubsection{The Magnetic Field Of One Magnetic Moment}

Each magnetic moment $\overrightarrow{\mu_{k}}$, independent of its source, has a magnetic vector potential $\overrightarrow{A_{k}}(\vec{r})$ [Neumann1848] which depends only on the strength of $\overrightarrow{\mu_{k}}$ and the vector $\vec{r}$ between an arbitrary point in space and $\overrightarrow{\mu_{k}}$. The indices $k$ and $l$ are used to distinguish between two different sources. $\times$ indicates the cross product between two vectors and $r$ is the length of the vector $\vec{r}$.

$$
\overrightarrow{A_{k}}(\vec{r})=\frac{\mu_{0}}{4 \pi} \frac{\overrightarrow{\mu_{k}} \times \vec{r}}{r^{3}}
$$

To determine the magnetic field $\overrightarrow{B_{k}}(\vec{r})$, which is generated by a magnetic moment $\overrightarrow{\mu_{k}}$, the curl of the vector potential is calculated. The curl of a vector field is the cross product between the Nabla operator and the vector field and generates a new vector field. $\overrightarrow{\mu_{k}} \cdot \vec{r}$ is the dot product between the two vectors.

$$
\overrightarrow{B_{k}}(\vec{r})=\nabla \times \overrightarrow{A_{k}}(\vec{r})=\left(\begin{array}{c}
\frac{\partial}{\partial x} \\
\frac{\partial}{\partial y} \\
\frac{\partial}{\partial z}
\end{array}\right) \times\left(\frac{\mu_{0}}{4 \pi} \frac{\overrightarrow{\mu_{k}} \times \vec{r}}{r^{3}}\right)=\frac{\mu_{0}}{4 \pi}\left(\frac{3 \vec{r}\left(\overrightarrow{\mu_{k}} \cdot \vec{r}\right)}{r^{5}}-\frac{\overrightarrow{\mu_{k}}}{r^{3}}\right)
$$

\subsubsection{The Dipole-Dipole Interaction Hamiltonian}

The energy of a second magnetic moment $\overrightarrow{\mu_{l}}$ in the field generated by the first one $\overrightarrow{B_{k}}$ is given by the dot product of the two vectors:

$$
E_{k l}^{\mathrm{dip}}=-\overrightarrow{\mu_{l}} \cdot \overrightarrow{B_{k}}\left(\overrightarrow{r_{k l}}\right)=-\frac{\mu_{0}}{4 \pi}\left(\frac{3\left(\overrightarrow{\mu_{k}} \cdot \overrightarrow{r_{k l}}\right)\left(\overrightarrow{\mu_{l}} \cdot \overrightarrow{r_{k l}}\right)}{r_{k l}^{5}}-\frac{\overrightarrow{\mu_{k}} \cdot \overrightarrow{\mu_{l}}}{r_{k l}^{3}}\right)
$$

$\overrightarrow{r_{k l}}$ is the vector between the two magnetic moments (Fig:2.1). Eq:11 is the well-known dipole-dipole equation. The vector $\overrightarrow{r_{k l}}$ is not static in time and therefore the dipolar Hamiltonian for two arbitrary 


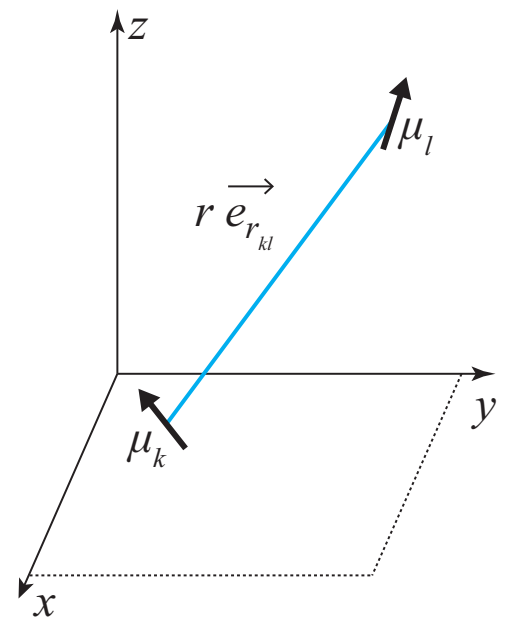

Figure 2.1: The vector between two magnetic moments in an arbitrary reference frame.

magnetic dipoles is time dependent and given by:

$$
\widehat{\mathscr{H}}_{k l}^{\operatorname{dip}}(t)=-\frac{\mu_{0}}{4 \pi r_{k l}^{3}}\left(3\left(\vec{\mu}_{k} \cdot \overrightarrow{e_{r_{k l}}}(t)\right)\left(\vec{\mu}_{l} \cdot \overrightarrow{e_{r_{k l}}}(t)\right)-\vec{\mu}_{k} \cdot \vec{\mu}_{l}\right)
$$

Following notation for vectors is used (Fig:2.2): $\overrightarrow{r_{k l}}=r_{k l} \overrightarrow{r_{k l}}, r_{k l}$ is the distance between the two magnetic moments and $\overrightarrow{e_{r_{k l}}}$ is the unit vector of $\overrightarrow{r_{k l}}$. The dipolar interaction Hamiltonian is the basis for all following calculations and in a later section a different notation is derived.

\subsection{Residual Dipolar Coupling - RDC}

To calculate the dipolar coupling induced by paramagnetic alignment, first the dipolar coupling between two spin 1/2 nuclei is derived. Afterwards, the general expression for residual dipolar coupling, dependent on an alignment tensor, is given and finally the obtained expression is extended for paramagnetic induced alignment. This chapter is based on the following review articles [Bertini2002] [Kramer2004]. 


\subsubsection{Dipolar Coupling Between Two Nuclei With The Same Spin}

The magnetic moment of a nucleus is given by $\vec{\mu}=\gamma \hbar \vec{I}, \gamma$ is the gyromagnetic ratio, $\hbar$ the reduced Planck constant and $\vec{I}$ the spin of the nucleus. Residual dipolar couplings are given in frequency units. For two atomic nuclei the dipolar Hamiltonian is:

$$
\widehat{\mathscr{H}}_{k l}^{\operatorname{dip}}(t)=\mathrm{D}_{k l}^{\operatorname{dip}}\left(3\left(\vec{I}_{k} \cdot{\overrightarrow{e_{k l}}}_{(}(t)\right)\left(\vec{I}_{l} \cdot{\overrightarrow{e_{k l}}}_{\vec{k}}(t)\right)-\vec{I}_{k} \cdot \vec{I}_{l}\right)
$$

$\mathrm{D}_{k l}^{\mathrm{dip}}=-\frac{1}{2 \pi} \frac{\mu_{0}}{4 \pi} \frac{\gamma_{k} \gamma_{l} \hbar}{r_{k l}^{3}}$ is the dipolar coupling constant. In a strong external magnetic field $\overrightarrow{B_{0}}$, the magnetic moments are quantized along $\overrightarrow{e_{B_{0}}}$ and only the secular part of the dipole coupling Hamiltonian is used (Eq:55), because only the secular term contributes to the energy that is dominated by the Zeeman interaction. The time dependency of the Hamiltonian is given by the variable angle $\theta$ (Fig: 2.2 ).

$$
\widehat{\mathscr{H}}_{k l}^{\operatorname{dip}}(t)=\mathrm{D}_{k l}^{\operatorname{dip}}\left(3 \cos ^{2}[\theta]-1\right)\left(I_{k z} I_{l z}-\frac{1}{2}\left(I_{k x} I_{l x}+I_{k y} I_{l y}\right)\right)
$$

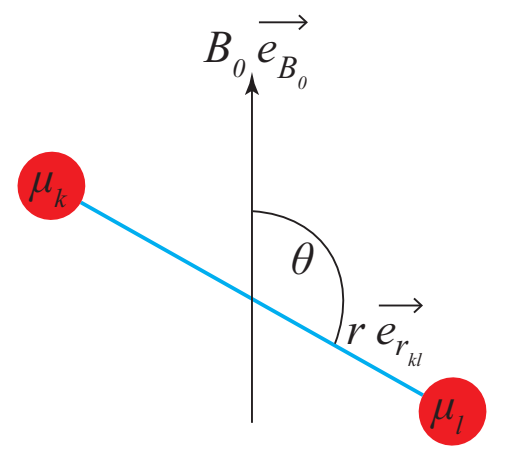

Figure 2.2: Definition of the angle between the external magnetic field and two magnetic moments.

The last two terms correspond to so-called 'flip-flop' transitions. In solution NMR, normally couplings between heteronuclei are measured $\left({ }^{13} \mathrm{C}-{ }^{1} \mathrm{H}\right.$ or $\left.{ }^{15} \mathrm{~N}-{ }^{1} \mathrm{H}\right)$ and the 'flip-flop' transitions in Eq:14 can be neglected because of the different Larmor frequencies making them non-secular. For identical spins with similar chemical shifts, the 'flip-flop' terms can just be appended since its angular dependency is identical to the $I_{k z} I_{l z}$ term. This results in the following Hamiltonian:

$$
\widehat{\mathscr{H}}_{k l}^{\operatorname{dip}}(t)=\mathrm{D}_{k l}^{\mathrm{dip}}\left(I_{k z} I_{l z}\right)\left(3 \cos ^{2}[\theta]-1\right)=\mathrm{D}_{k l}^{\operatorname{dip}}\left(I_{k z} I_{l z}\right)\left(3\left(\overrightarrow{e_{B_{0}}} \cdot \overrightarrow{e_{r_{k l}}}(t)\right)\left(\overrightarrow{e_{B_{0}}} \cdot \overrightarrow{e_{r_{k l}}}(t)\right)-\overrightarrow{e_{B_{0}}} \overrightarrow{e_{B_{0}}}\right)
$$

$\mathrm{D}_{k l}^{\mathrm{dip}}$ is the maximum observable value for residual dipolar couplings. The latter part of Eq:15 is the scaling of this maximum value for different orientations of the internuclear vector with respect 
to the external magnetic field. For solution state NMR, the random orientation of molecules in the sample can be described by the movement of the vector of the external magnetic field in an arbitrary coordinate system in the molecular frame $(x, y, z)$ (Fig:2.3). Note the change of the angle $\theta$ due to the change of the reference frame. The vector of the external magnetic field is time dependent in this reference frame.

$$
\overrightarrow{{e_{B}}_{0}}(t)=\left(\begin{array}{c}
B_{x}(t) \\
B_{y}(t) \\
B_{z}(t)
\end{array}\right)=\left(\begin{array}{c}
\cos [\beta] \sin [\alpha] \\
\sin [\beta] \sin [\alpha] \\
\cos [\alpha]
\end{array}\right) \quad \overrightarrow{e_{r_{k l}}}=\left(\begin{array}{c}
\cos [\varphi] \sin [\theta] \\
\sin [\varphi] \sin [\theta] \\
\cos [\theta]
\end{array}\right)
$$

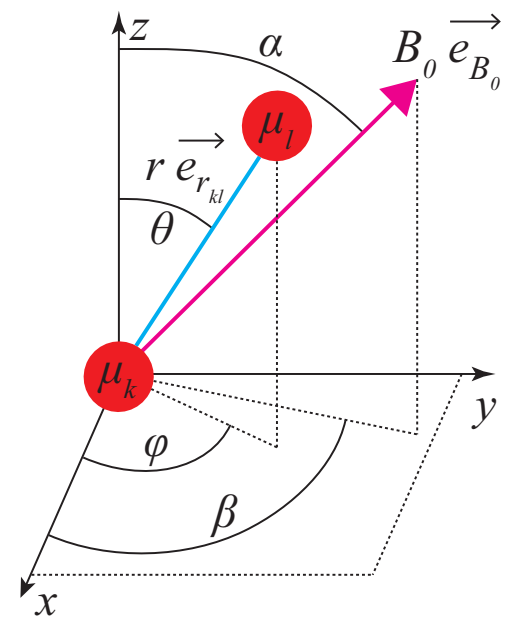

Figure 2.3: The external magnetic field and the vector between two magnetic moments in an arbitrary coordinate system.

The scaled value $\mathrm{D}_{k l}^{\mathrm{RDC}}$ in the new reference frame is given by:

$$
\left.\begin{array}{rl}
\mathrm{D}_{k l}^{\mathrm{RDC}} & =\mathrm{D}_{k l}^{\mathrm{dip}}\left(3\left(\overrightarrow{e_{B_{0}}}(t) \cdot \overrightarrow{e_{r_{k l}}}\right)\left(\overrightarrow{e_{B_{0}}}(t) \cdot \overrightarrow{e_{r_{k l}}}\right)-\overrightarrow{e_{B_{0}}}(t) \overrightarrow{e_{B_{0}}}(t)\right) \\
& =\mathrm{D}_{k l}^{\mathrm{dip}}\left(3\left(\overrightarrow{e_{r_{k l}}} \cdot\left(\overrightarrow{e_{B_{0}}}(t) \otimes \overrightarrow{e_{B_{0}}}(t)\right) \cdot \overrightarrow{e_{r_{k}}}\right)-1\right) \\
& =\mathrm{D}_{k l}^{\operatorname{dip}}\left(3\left(\overrightarrow{e_{r_{k l}}} \cdot\left(\begin{array}{lll}
B_{x}(t) B_{x}(t) & B_{x}(t) B_{y}(t) & B_{x}(t) B_{z}(t) \\
B_{x}(t) B_{y}(t) & B_{y}(t) B_{y}(t) & B_{y}(t) B_{z}(t) \\
B_{x}(t) B_{z}(t) & B_{y}(t) B_{z}(t) & B_{z}(t) B_{z}(t)
\end{array}\right)-\overrightarrow{e_{r_{k l}}}\right)-1\right)
\end{array}\right)
$$

The dot product of two identical unit vectors is 1 and $\otimes$ indicates the Kronecker product of two vectors. In solution state NMR, measuring molecules tumbling with nano-seconds correlation times, 
only an averaged value $\overline{\mathrm{D}_{k l}^{\mathrm{RDC}}}$ is observed given by:

$$
\begin{aligned}
\overrightarrow{\mathrm{D}_{k l}^{\mathrm{RDC}}} & =\mathrm{D}_{k l}^{\operatorname{dip}}\left(3\left(\overrightarrow{e_{r_{k l}} \cdot} \cdot\left(\begin{array}{lll}
\overrightarrow{B_{x}(t) B_{x}(t)} & \overline{B_{x}(t) B_{y}(t)} & \overline{B_{x}(t) B_{z}(t)} \\
\overline{B_{x}(t) B_{y}(t)} & \overline{B_{y}(t) B_{y}(t)} & \overline{B_{y}(t) B_{z}(t)} \\
B_{y}(t) B_{z}(t) & \overline{B_{z}(t) B_{z}(t)}
\end{array}\right) \cdot \overrightarrow{e_{r_{k l}}}\right)-1\right) \\
& =\mathrm{D}_{k l}^{\operatorname{dip}}\left(3\left(\overrightarrow{e_{r_{k l}}} \cdot \boldsymbol{P} \cdot \overrightarrow{e_{r_{k l}}}\right)-1\right)
\end{aligned}
$$

The matrix $\boldsymbol{P}$ is called probability matrix, is real, symmetric and has a trace of 1 . With these properties, there are just five independent parameters and it can be diagonalized. ${\overline{B_{x}(t)}}^{2}$ indicates the probability to find the $x$-axis of an arbitrary molecule coordinate system aligned with the external magnetic field $\overrightarrow{B_{0}}$. For example, if ${\overrightarrow{B_{x}(t)}}^{2}$ is perfectly aligned with the external magnetic field, it would be 1 and the other two values would be 0 .

$$
\boldsymbol{P}_{\text {diag }}=\left(\begin{array}{ccc}
{\overline{B_{x}(t)}}^{2} & 0 & 0 \\
0 & {\overline{B_{y}(t)}}^{2} & 0 \\
0 & 0 & {\overline{B_{z}(t)}}^{2}
\end{array}\right)
$$

$\boldsymbol{P}$ can be visualized as an ellipsoid, but the differences to a perfect sphere are too small to be seen (Fig.2.4). The alignment tensor $\boldsymbol{A}$ is definied as the difference between $\boldsymbol{P}_{\text {diag }}$ and the unity matrix $\mathbf{1}$ :

$$
\boldsymbol{A}=\left(\begin{array}{ccc}
A_{x x} & 0 & 0 \\
0 & A_{y y} & 0 \\
0 & 0 & A_{z z}
\end{array}\right)=\boldsymbol{P}_{\text {diag }}-\frac{1}{3} \mathbf{1}=\left(\begin{array}{ccc}
{\overline{B_{x}(t)}}^{2} & 0 & 0 \\
0 & \bar{B}_{y}(t) & 0 \\
0 & 0 & \bar{B}_{z}(t)^{2}
\end{array}\right)-\frac{\mathbf{1}}{\mathbf{3}}\left(\begin{array}{ccc}
1 & 0 & 0 \\
0 & 1 & 0 \\
0 & 0 & 1
\end{array}\right)
$$

For example, if the $x$-direction of a molecule is perfectly aligned with the $B_{0}$-field, the value of $A_{x x}$ would be $2 / 3$ and the other two would be $-1 / 3$. $\boldsymbol{A}$ is real, symmetric, traceless and if $\boldsymbol{P}$ is expressed in its diagonalized form, $\boldsymbol{A}$ is also diagonalized. For the diagonalized form following convention is used: $A_{z z}>A_{x x}>A_{y y}$. With Eq:20 the RDC is given by:

$$
\begin{aligned}
\overrightarrow{\mathrm{D}_{k l}^{\mathrm{RDC}}} & =\mathrm{D}_{k l}^{\mathrm{dip}}\left(3\left(\overrightarrow{e_{r_{k l}}} \cdot \boldsymbol{P} \cdot \overrightarrow{e_{r_{k l}}}\right)-1\right) \\
& =\mathrm{D}_{k l}^{\mathrm{dip}} 3\left(\overrightarrow{e_{r_{k l}}} \cdot \boldsymbol{A} \cdot \overrightarrow{e_{r_{k l}}}\right)
\end{aligned}
$$

Without alignment the molecule is randomly tumbling in solution and thus all elements in $\boldsymbol{A}$ and $\overrightarrow{\mathrm{D}_{k l}^{\mathrm{RDC}}}$ are 0 . A non-zero alignment tensor results in residual dipolar couplings. With $\overrightarrow{e_{r_{k l}}}$ expressed in 
spherical coordinates $\left(\mathrm{Eq}:[16), \sin ^{2}[\varphi]=\left(\frac{1}{2}-\frac{1}{2} \cos [2 \varphi]\right)\right.$ and $\cos ^{2}[\varphi]=\left(\frac{1}{2}+\frac{1}{2} \cos [2 \varphi]\right)$ the residual dipolar coupling $\overline{\mathrm{D}_{k l}^{\mathrm{RDC}}}$ is given by:

$$
\begin{aligned}
\overrightarrow{\mathrm{D}_{k l}^{\mathrm{RDC}}} & =\mathrm{D}_{k l}^{\mathrm{dip}_{3}} 3\left(\overrightarrow{e_{r_{k l}}} \cdot \boldsymbol{A} \cdot \overrightarrow{e_{r_{k l}}}\right) \\
& =\mathrm{D}_{k l}^{\operatorname{dip}_{3}} 3\left(\left(\begin{array}{c}
\cos [\varphi] \sin [\theta] \\
\sin [\varphi] \sin [\theta] \\
\cos [\theta]
\end{array}\right) \cdot\left(\begin{array}{ccc}
A_{x x} & 0 & 0 \\
0 & A_{y y} & 0 \\
0 & 0 & A_{z z}
\end{array}\right) \cdot\left(\begin{array}{c}
\cos [\varphi] \sin [\theta] \\
\sin [\varphi] \sin [\theta] \\
\cos [\theta]
\end{array}\right)\right) \\
& =-\frac{1}{2 \pi} \frac{\mu_{0}}{4 \pi} \frac{\gamma_{k} \gamma_{l} \hbar}{r_{k l}^{3}} 3\left(\frac{1}{2} A_{z z}\left(3 \cos ^{2}[\theta]-1\right)+\frac{1}{2}\left(A_{x x}-A_{y y}\right) \cos [2 \varphi] \sin ^{2}[\theta]\right)
\end{aligned}
$$

An example for an alignment tensor, corresponding to weak alignment with a paramagnetic $\operatorname{tag}\left(A_{z z}=\right.$ $6.9834 \cdot 10^{-3}, A_{x x}=-2.7143 \cdot 10^{-3}, A_{y y}=-4.2691 \cdot 10^{-3}$ ), and the corresponding probability matrix are shown in Fig.2.4
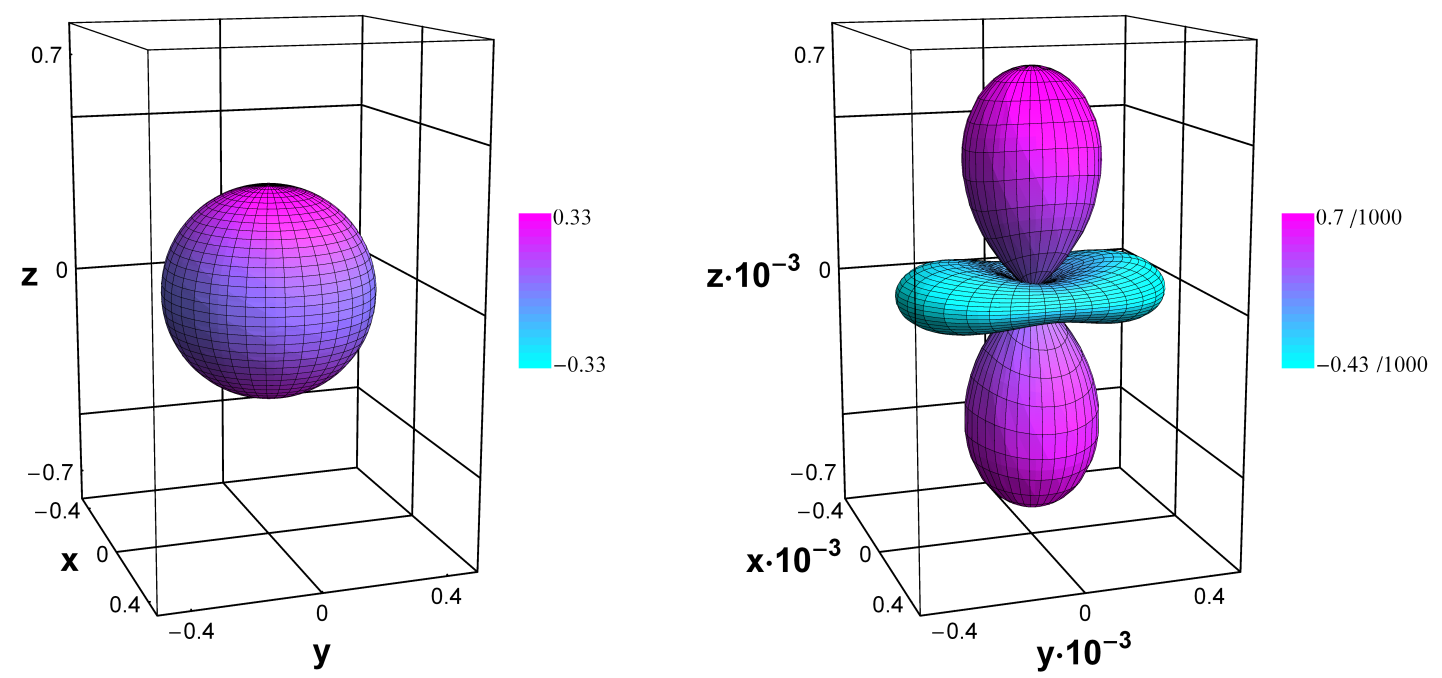

Figure 2.4: Examples for a probability matrix (left) and an alignment tensor (right).

Alignment of molecules can be achieved with different methods. External alignment media like phages, bicelles or gels usually lead to very strong alignments. Another form is auto-alignment of molecules if they have an anisotropic magnetic susceptibility tensor. This is true for big DNA strands due to the $\pi$-stacking of the nucleobases [Kung1995] [Al-Hashimi2001a] [Al-Hashimi2001b] [Bryce2004] or for molecules with paramagnetic centers. These centers can be stable radicals or paramagnetic ions and occur naturally or are inserted in molecules synthetically with paramagnetic 
tags [Gaponenko2002] [Bertini2003] [Woehnert2003] [Rodriguez2006] [Su2010] [Dasgupta2011]. Alignment tensors are usually back calculated with experimental data and the quality of this calculation is given by two different numbers ( $q$-factor and $R^{2}$-value):

$$
q=\sqrt{\frac{\sum\left(v_{\exp }-v_{\mathrm{cal}}\right)^{2}}{\sum\left(v_{\exp }\right)^{2}}} \quad R^{2}=1-\frac{\sum\left(v_{\exp }-v_{\mathrm{cal}}\right)^{2}}{\sum\left(v_{\exp }-\overline{v_{\exp }}\right)^{2}}
$$

$v_{\text {exp }}$ are the experimental values, $v_{\text {cal }}$ the corresponding back calculated values and $\overline{v_{\exp }}$ is the average value of the experimental values. The $q$-factor is best when closely to 0 and the $R^{2}$-value is best when closely to 1 .

\subsubsection{Anisotropic Magnetic Susceptibility And The Magnetic Field Dependency Of RDC}

To understand why anisotropic magnetic susceptibility causes alignment of molecules, it is necessary to derive the correlation between the external magnetic field and the average magnetic moment induced by the anisotropic magnetic susceptibility. The magnetic field $\overrightarrow{B_{0}}$ is the magnetic field intensity $\overrightarrow{H_{0}}$ multiplied with the vacuum permeability $\mu_{0}$. The magnetization $\vec{M}$ of a substance is defined as the induced magnetic moment $\vec{\mu}$ per volume $V$ [Bennett1978].

$$
\overrightarrow{B_{0}}=\mu_{0} \overrightarrow{H_{0}} \quad \overrightarrow{H_{0}}=\frac{1}{\mu_{0}} \overrightarrow{B_{0}} \quad \vec{M}=\frac{1}{V} \vec{\mu}
$$

The magnetic susceptibility per volume is related to the magnetization with:

$$
\vec{M}=\chi_{V} \overrightarrow{H_{0}}
$$

This leads to:

$$
\vec{M}=\frac{1}{V} \vec{\mu}=\chi_{V} \overrightarrow{H_{0}}=\frac{1}{\mu_{0}} \chi_{V} \overrightarrow{B_{0}} \quad \vec{\mu}=\frac{1}{\mu_{0}} \chi \overrightarrow{B_{0}} \quad \mu_{z}=\frac{1}{\mu_{0}} \chi B_{0}
$$

$\chi_{V}$ is the magnetic susceptibility per volume and $\chi$ per molecule.

To avoid confusion in the next sections, at this point, the magnetic moments associated with paramagnetic centers are described. This section follows the review article [Bertini2002]. For a single electron the magnetic moment is:

$$
\vec{\mu}_{S}=-\mu_{B} g_{e} \vec{S}
$$


Included in this complete description, there is the magnetic moment which is induced by the external magnetic field along its direction across the sample (Eq:26). The average induced magnetic moment per molecule $\left\langle\mu_{S_{z}}\right\rangle$ can be calculated with the expectation value of the spin operator $\left\langle S_{z}\right\rangle$ :

$$
\left\langle\mu_{S_{z}}\right\rangle=-\mu_{B} g_{e}\left\langle S_{z}\right\rangle
$$

For electrons in a strong external magnetic field, there are $2 S+1$ different states whose energies are given by $E_{S_{z}}=\mu_{B} g_{e} S_{z} B_{0}$, where $S_{z}$ is the $z$-component of the spin for the different states. The expectation value of $S_{z}$ can be calculated with Boltzmann statistics:

$$
\left\langle\mu_{S_{z}}\right\rangle=-\mu_{B} g_{e} \frac{\sum_{S_{z}=-S}^{S} S_{z} \exp \left[-\frac{\mu_{B} g_{e} S_{z} B_{0}}{k T}\right]}{\sum_{S_{z}=-S}^{S} \exp \left[-\frac{\mu_{B} g_{e} S_{z} B_{0}}{k T}\right]}=-\mu_{B} g_{e} \frac{\sum_{S_{z}=-S}^{S} S_{z}\left(1-\frac{\mu_{B} g_{e} S_{z} B_{0}}{k T}\right)}{\sum_{S_{z}=-S}^{S}\left(1-\frac{\mu_{B} g_{e} S_{z} B_{0}}{k T}\right)}
$$

The exponential term is approximated to first order since with strong magnetic fields and temperatures above a few Kelvin $\mu_{B} g_{e} S_{z} B_{0} \ll k T$.

$$
\begin{aligned}
\left\langle\mu_{S_{z}}\right\rangle & =-\mu_{B} g_{e} \frac{\sum_{S_{z}=-S}^{S}-\frac{\mu_{B} g_{e} S_{z}^{2} B_{0}}{k T}}{2 S+1}=\frac{\mu_{B}^{2} g_{e}^{2} B_{0}}{k T} \frac{\sum_{S_{z}=0}^{S} 2 S_{z}^{2}}{2 S+1} \\
& =\frac{\mu_{B}^{2} g_{e}^{2} B_{0}}{k T} \frac{2 S(S+1)(2 S+1)}{6(2 S+1)}=\frac{\mu_{B}^{2} g_{e}^{2} B_{0}}{3 k T} S(S+1)
\end{aligned}
$$

This is Curie's law, the magnetic moment named Curie spin and combining Eq:30 and Eq:26 results in following expression for the magnetic susceptibility:

$$
\chi=\frac{\mu_{0} \mu_{B}^{2} g_{e}^{2}}{3 k T} S(S+1)
$$

If the electron orbital momentum is considered, it is assumed that the system is sufficiently described

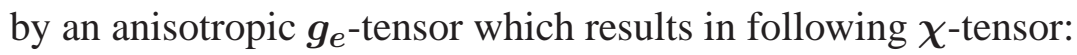

$$
\chi=\frac{\mu_{0} \mu_{B}^{2} g_{e}^{2}}{3 k T} S(S+1)
$$

This tensor is of second rank and defines the magnetic susceptibility coordinate system of a molecule and, similar to the alignment tensor, is real, symmetric and thus it can be expressed in its diagonalized form. The consequence of an anisotropic magnetic susceptibility tensor is that the average induced magnetic moment is no longer parallel to the external magnetic field and given by:

$$
\left\langle\overrightarrow{\mu_{S}}\right\rangle=\frac{1}{\mu_{0}} \chi \cdot \overrightarrow{B_{0}}
$$


To calculate the energy $E$ which is induced by the magnetic susceptibility, integration over $\mathrm{d}\left\langle\overrightarrow{\mu_{S}}\right\rangle$ has to be performed, because the magnetic moment changes relatively to the direction of the external magnetic field [Bothner-By1996].

$$
E=-\left\langle\overrightarrow{\mu_{S}}\right\rangle \cdot \overrightarrow{B_{0}}=-\int_{0}^{\left\langle\overrightarrow{\mu_{S}}\right\rangle} \overrightarrow{B_{0}} \mathrm{~d}\left\langle\overrightarrow{\mu_{S}}\right\rangle=-\frac{\overrightarrow{B_{0}} \cdot \chi \cdot \overrightarrow{B_{0}}}{2 \mu_{0}}
$$

This orientation dependent energy, induced by the anisotropic magnetic susceptibility, is the origin of a preferred orientation of the molecule, known as alignment. With a diagonalized $\chi$-tensor and the external magnetic field in spherical coordinates for the $z$-axis of this coordinate frame (Eq:16) the energy is given by :

$$
\begin{aligned}
E_{z z} & =-\frac{\overrightarrow{B_{0}} \cdot \chi \cdot \overrightarrow{B_{0}}}{2 \mu_{0}} \\
& =-\frac{B_{0}^{2}}{2 \mu_{0}}\left(\begin{array}{c}
\cos [\beta] \sin [\alpha] \\
\sin [\beta] \sin [\alpha] \\
\cos [\alpha]
\end{array}\right) \cdot\left(\begin{array}{ccc}
\chi_{x x} & 0 & 0 \\
0 & \chi_{y y} & 0 \\
0 & 0 & \chi_{z z}
\end{array}\right) \cdot\left(\begin{array}{c}
\cos [\beta] \sin [\alpha] \\
\sin [\beta] \sin [\alpha] \\
\cos [\alpha]
\end{array}\right) \\
& =-\frac{B_{0}^{2}}{2 \mu_{0}}\left(\chi_{y y} \sin ^{2}[\alpha] \sin ^{2}[\beta]+\chi_{x x} \sin ^{2}[\alpha] \cos ^{2}[\beta]+\chi z \cos ^{2}[\alpha]\right)
\end{aligned}
$$

The alignment of the axes of the $\chi$-tensor to the external magnetic field can be calculated with Boltzmann statistics.

$$
A_{z z}=\frac{2}{3} \frac{\int\left(\frac{3 \cos ^{2}[\alpha]-1}{2}\right) \exp \left[-\frac{E}{k T}\right]}{\int \exp \left[-\frac{E}{k T}\right]}
$$

The factor $2 / 3$ is a scaling factor for the alignment thus that a perfect alignment of an axis is again represented by: $A_{z z}=2 / 3 \cdot \frac{3 \cos ^{2}[\alpha]-1}{2}$ is the density of states, the denominator the partition function, $k$ the Boltzmann constant and $T$ the temperature. The exponential term can be approximated to first order because $E \ll k T$. The integration over the spherical angles leads to the additional term $\sin [a]$ and the normalization factor of $\frac{1}{4 \pi}$. Henceforth, only the anisotropic part of the $\chi$-tensor is considered $\left(\chi_{x x}+\chi_{y y}+\chi_{z z}=0\right)$, because only the anisotropy induces observable changes in the NMR spectra.

$$
\begin{aligned}
A_{z z} & =\frac{\frac{1}{4 \pi} \int_{0}^{2 \pi} \int_{0}^{\pi} \frac{3 \cos ^{2}[\alpha]-1}{2}\left(1-\frac{E}{k T}\right) \sin [\alpha] \mathrm{d} \alpha \mathrm{d} \beta}{\frac{1}{4 \pi} \int_{0}^{2 \pi} \int_{0}^{\pi}\left(1-\frac{E}{k T}\right) \sin [\alpha] \mathrm{d} \alpha \mathrm{d} \beta} \\
& =\frac{2}{3} \frac{B_{0}^{2}}{15 \mu_{0} k T}\left(\chi_{z z}-\frac{\chi_{x x}+\chi_{y y}}{2}\right)
\end{aligned}
$$


The parameter for the other two axes of the $\chi$-tensor coordinate system are obtained by similar calculations with the external magnetic field in spherical coordinates for the respective axis:

$$
A_{x x}=\frac{2}{3} \frac{B_{0}^{2}}{15 \mu_{0} k T}\left(\chi_{x x}-\frac{\chi_{z z}+\chi_{y y}}{2}\right) \quad A_{y y}=\frac{2}{3} \frac{B_{0}^{2}}{15 \mu_{0} k T}\left(\chi_{y y}-\frac{\chi_{z z}+\chi_{x x}}{2}\right)
$$

With this and recalling Eq:22 the residual dipolar coupling is completely described by:

$$
\begin{aligned}
\overline{\mathrm{D}_{k l}^{\mathrm{RDC}}}= & -\frac{1}{2 \pi} \frac{\mu_{0}}{4 \pi} \frac{\gamma_{k} \gamma_{l} \hbar}{r_{k l}^{3}} 3\left(\frac{1}{2} \frac{2}{3} \frac{B_{0}^{2}}{15 \mu_{0} k T}\left(\chi_{z z}-\frac{\chi_{x x}+\chi_{y y}}{2}\right)\left(3 \cos ^{2}[\theta]-1\right)\right. \\
& \left.+\frac{1}{2}\left(\frac{2}{3} \frac{B_{0}^{2}}{15 \mu_{0} k T}\left(\chi_{x x}-\frac{\chi_{z z}+\chi_{y y}}{2}\right)-\frac{2}{3} \frac{B_{0}^{2}}{15 \mu_{0} k T}\left(\chi_{y y}-\frac{\chi_{z z}+\chi_{x x}}{2}\right)\right) \cos [2 \varphi] \sin ^{2}[\theta]\right) \\
= & -\frac{1}{8 \pi^{2}} \frac{\gamma_{k} \gamma_{l} \hbar}{r_{k l}^{3}} \frac{B_{0}^{2}}{15 \mu_{0} k T}\left(\left(3 \cos ^{2}[\theta]-1\right)\left(\chi_{z z}-\frac{\chi_{x x}+\chi_{y y}}{2}\right)\right. \\
& \left.+\frac{3}{2}\left(\chi_{x x}-\chi_{y y}\right) \cos [2 \varphi] \sin ^{2}[\theta]\right)
\end{aligned}
$$

Eq 39 indicates all parameter which are constraints for residual dipolar couplings. On one hand, there are the magnetic field dependency and the gyromagnetic ratios of the nuclei which are known and on the other hand, the magnetic susceptibility tensor describing the molecular frame which is usually back calculated with experimental data. The last constraints are the distance between the two nuclei and the angles of the inter-nuclear vector in the molecular frame which are important structural information.

\subsubsection{Sauper Order Matrix}

For completeness, another form for a description of the movement of the magnetic field in the molecular coordinate system is given. In the literature, the Saupe order matrix $S$ (Fig.2.5) is often used to describe this movement in spherical coordinates [Saupe1968]. $S_{i i}$ gives the probability to find the external magnetic field along the axes of this coordinate system. A value of 1 means that this axis is perfectly aligned to $\overrightarrow{B_{0}}$, whereas a value of $-1 / 2$ means that the axis is always perfect perpendicular to $\overrightarrow{B_{0}}$. 


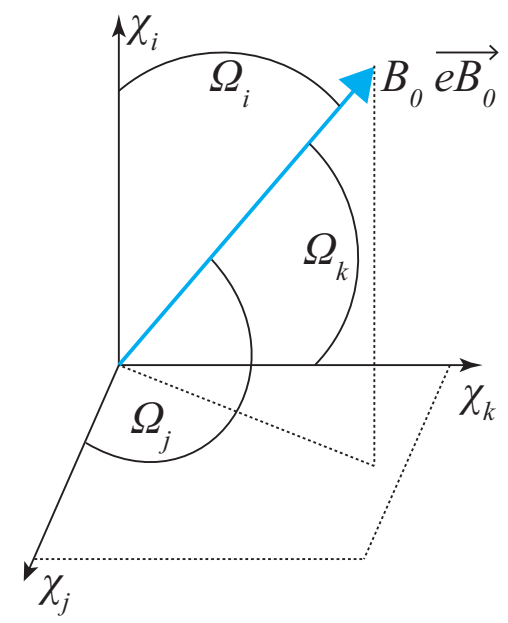

Figure 2.5: Definition of the angles for the Saupe matrix.

$$
\begin{aligned}
\boldsymbol{S} & =\frac{1}{2}\left\langle 3 \cos \left[\Omega_{a}\right] \cos \left[\Omega_{b}\right]-\delta_{a b}\right\rangle \\
& =\frac{1}{2}\left\langle\left(\begin{array}{ccc}
3 \cos ^{2}\left[\Omega_{i}\right]-1 & 3 \cos \left[\Omega_{i}\right] \cos \left[\Omega_{j}\right] & 3 \cos \left[\Omega_{i}\right] \cos \left[\Omega_{k}\right] \\
3 \cos \left[\Omega_{i}\right] \cos \left[\Omega_{j}\right] & 3 \cos ^{2}\left[\Omega_{j}\right]-1 & 3 \cos \left[\Omega_{j}\right] \cos \left[\Omega_{k}\right] \\
3 \cos \left[\Omega_{i}\right] \cos \left[\Omega_{k}\right] & 3 \cos \left[\Omega_{j}\right] \cos \left[\Omega_{k}\right] & 3 \cos ^{2}\left[\Omega_{k}\right]-1
\end{array}\right)\right\rangle
\end{aligned}
$$

$\delta_{a b}$ is the Kronecker delta, the angle brackets stand for the time average and $a, b=i, j, k$. The obtained expressions have to be scaled because of the different values for a perfect alignment of the alignment tensor (2/3) and the Saupe order matrix (1):

$$
A=\frac{2}{3} S
$$

This factor (2/3) is important when comparing equations which use different notations.

\subsection{Pseudocontact Shifts - PCS}

Paramagnetic centers not only induce alignment but also change the chemical shifts of the nuclei. The origin of the effect is the anisotropy of the susceptibility tensor which not only leads to an orientation dependent alignment but also to an orientation dependent strength of the dipolar coupling between the paramagnetic center $(S)$ and the nucleus $(I)$. In contrast to residual dipolar couplings this effect, known as pseudocontact shift, occurs independently of alignment. 


\subsubsection{Interaction Of Nucleus And The Paramagnetic Center}

Considering the $\chi$-tensor in its diagonalized form and recalling Eq:11 (the secular term of the dipolar interaction Hamiltonian), the energy of the interaction between a nucleus $\overrightarrow{\mu_{I}}=\gamma_{I} \hbar I_{z} \overrightarrow{B_{0}}(t)$ and the average induced magnetic moment of a paramagnetic center $\left\langle\overrightarrow{\mu_{S}}\right\rangle=\frac{1}{\mu_{0}} \chi \cdot \overrightarrow{B_{0}}(t)$ is given by:

$$
E_{\mathrm{PCS}}(t)=-\frac{\gamma_{I} \hbar I_{z} B_{0}}{4 \pi r_{I S}^{3}}\left(3\left(\left(\chi \cdot \overrightarrow{e_{B_{0}}}(t)\right) \cdot \overrightarrow{e_{r_{I S}}}\right)\left(\overrightarrow{e_{B_{0}}}(t) \cdot \overrightarrow{e_{r_{I S}}}\right)-\left(\chi \cdot \overrightarrow{e_{B_{0}}}(t)\right) \cdot\left(\overrightarrow{e_{B_{0}}}(t)\right)\right)
$$

As well as for the description of the alignment, for this interaction only the anisotropic part of the $\chi$-tensor is considered, because the isotropic part is averaged to zero due to the rapid tumbling of the molecule. Furthermore, if the rotational correlation time is faster than the longitudinal relaxation time of the electron, the effects of the anisotropy are averaged to 0 . This interaction contributes to the energy difference between the states of the nucleus which are quantized along the external magnetic field. For a spin $1 / 2$ nucleus this is given by: $\Delta I_{z}=I_{\alpha}-I_{\beta}=\frac{1}{2}-\left(-\frac{1}{2}\right)=1$. To change the dimension of this energy to ppm (parts per million) the factor $\frac{10^{6}}{\gamma_{I} \hbar B_{0}}$ is introduced. The random motion of the molecule is considered by integration over the spherical angles of the external magnetic field. Recalling Eq 16 the pseudocontact shift is given by:

$$
\begin{aligned}
& \delta_{\mathrm{PCS}}= \\
& \int_{0}^{2 \pi} \int_{0}^{\pi}\left(3\left(\chi \cdot\left(\begin{array}{c}
\cos [\beta] \sin [\alpha] \\
\sin [\beta] \sin [\alpha] \\
\cos [\alpha]
\end{array}\right) \cdot\left(\begin{array}{c}
\cos [\varphi] \sin [\theta] \\
\sin [\varphi] \sin [\theta] \\
\cos [\theta]
\end{array}\right)\right)\left(\left(\begin{array}{c}
\cos [\beta] \sin [\alpha] \\
\sin [\beta] \sin [\alpha] \\
\cos [\alpha]
\end{array}\right) \cdot\left(\begin{array}{c}
\cos [\varphi] \sin [\theta] \\
\sin [\varphi] \sin [\theta] \\
\cos [\theta]
\end{array}\right)\right)\right. \\
& \left.-\left(\chi \cdot\left(\begin{array}{c}
\cos [\beta] \sin [\alpha] \\
\sin [\beta] \sin [\alpha] \\
\cos [\alpha]
\end{array}\right)\right) \cdot\left(\begin{array}{c}
\cos [\varphi] \sin [\theta] \\
\sin [\varphi] \sin [\theta] \\
\cos [\theta]
\end{array}\right)\right) \sin [\alpha] \mathrm{d} \alpha \mathrm{d} \beta \frac{1}{4 \pi} 10^{6} \frac{1}{4 \pi r_{I S}^{3}} \\
& =\frac{1}{12 \pi r_{I S}^{3}}\left(\left(3 \cos ^{2}[\theta]-1\right)\left(\begin{array}{c}
\chi_{x x}+\chi_{y y} \\
2
\end{array}\right)+\frac{3}{2}\left(\chi_{x x}-\chi_{y y}\right) \cos [2 \varphi] \sin ^{2}[\theta]\right) 10^{6}
\end{aligned}
$$

Eq:43 is the final expression for pseudocontact shifts caused by an anisotropic magnetic susceptibility tensor of a paramagnetic center. Comparing to Eq:39, pseudocontact shifts depend also on the magnetic susceptibility tensor but contrary to residual dipolar couplings, the distance and angles describe the vector between one nucleus and the paramagnetic center in the molecular frame which gives rise to the condition of knowing the position of the paramagnetic center in this frame. 


\subsubsection{Alignment Effects On The PCSs}

For the calculation of the magnetic field dependency of pseudocontact shifts an alternative derivation for the pseudocontact shifts, in terms of the dipolar interaction tensor, is given.

$$
\begin{aligned}
\delta_{\mathrm{PCS}} & =\frac{1}{4 \pi r_{I S}^{3}}\left(3\left(\left(\chi \cdot \overrightarrow{e_{B_{0}}}(t)\right) \cdot \overrightarrow{e_{r_{I S}}}\right)\left(\overrightarrow{e_{B_{0}}}(t) \cdot \overrightarrow{e_{r_{I S}}}\right)-\left(\chi \cdot \overrightarrow{e_{B_{0}}}(t)\right) \cdot\left(\overrightarrow{e_{B_{0}}}(t)\right)\right) 10^{6} \\
& =\frac{1}{4 \pi r_{I S}^{3}}\left(3 \overrightarrow{e_{B_{0}}}(t) \cdot\left(\chi \cdot \overrightarrow{e_{r_{I S}}}\right)\left(\overrightarrow{e_{r_{I S}}} \cdot \overrightarrow{e_{B_{0}}}(t)\right)-\left(\overrightarrow{e_{B_{0}}}(t) \cdot \chi \cdot \overrightarrow{e_{B_{0}}}(t)\right)\right) 10^{6} \\
& =\frac{1}{4 \pi r_{I S}^{3}}\left(\overrightarrow{e_{B_{0}}}(t) \cdot\left(3\left(\chi \cdot \overrightarrow{e_{r_{I S}}}\right) \otimes \overrightarrow{e_{r_{I S}}}-\chi\right) \cdot \overrightarrow{e_{B_{0}}}(t)\right) 10^{6} \\
& =\frac{1}{4 \pi r_{I S}^{3}}\left(\overrightarrow{e_{B_{0}}}(t) \cdot\left(3\left(\overrightarrow{e_{r_{I S}}} \otimes \overrightarrow{e_{r_{I S}}}\right) \cdot \chi-\chi\right) \cdot \overrightarrow{e_{B_{0}}}(t)\right) 10^{6} \\
& =\left(\overrightarrow{e_{B_{0}}}(t) \cdot \sigma \cdot \overrightarrow{e_{B_{0}}}(t)\right) 10^{6}
\end{aligned}
$$

$\sigma$ is the dipolar interaction tensor:

$$
\begin{aligned}
\sigma & =\frac{1}{4 \pi r_{I S}^{3}}\left(3\left(\overrightarrow{e_{r_{I S}}} \otimes \overrightarrow{e_{r_{I S}}}\right) \cdot \chi-\chi\right) \\
& =\frac{1}{4 \pi r_{I S}^{3}}\left(\begin{array}{ccc}
\chi_{x x}\left(3 \sin ^{2}[\theta] \cos ^{2}[\varphi]-1\right) & \chi_{y y} 3 \sin ^{2}[\theta] \cos [\varphi] \sin [\varphi] & \chi_{z z} 3 \cos [\theta] \sin [\theta] \cos [\varphi] \\
\chi_{x x} 3 \sin ^{2}[\theta] \cos [\varphi] \sin [\varphi] & \chi_{y y}\left(3 \sin ^{2}[\theta] \sin ^{2}[\varphi]-1\right) & \chi_{z z} 3 \sin [\theta] \cos [\theta] \sin [\varphi] \\
\chi_{x x} 3 \cos [\theta] \sin [\theta] \cos [\varphi] & \chi_{y y} 3 \sin [\theta] \cos [\theta] \sin [\varphi] & \chi_{z z}\left(3 \cos ^{2}[\theta]-1\right)
\end{array}\right)
\end{aligned}
$$

The average rotation of the magnetic field can be calculated by expressing the unit vector of the magnetic field as a linear combination of the three principal axes divided by three. With this the pseudocontact shift is given by:

$$
\begin{aligned}
& \delta_{\mathrm{PCS}}=\left(\overrightarrow{e_{B_{0}}}(t) \cdot \boldsymbol{\sigma} \cdot \overrightarrow{e_{B_{0}}}(t)\right) 10^{6} \\
& =\left(\frac{1}{3}\left(\left(\begin{array}{l}
1 \\
0 \\
0
\end{array}\right)+\left(\begin{array}{l}
0 \\
1 \\
0
\end{array}\right)+\left(\begin{array}{l}
0 \\
0 \\
1
\end{array}\right)\right)\right) \cdot \sigma \cdot\left(\frac{1}{3}\left(\left(\begin{array}{l}
1 \\
0 \\
0
\end{array}\right)+\left(\begin{array}{l}
0 \\
1 \\
0
\end{array}\right)+\left(\begin{array}{l}
0 \\
0 \\
1
\end{array}\right)\right)\right) 10^{6} \\
& =\frac{1}{3}\left(\left(\begin{array}{l}
1 \\
0 \\
0
\end{array}\right) \cdot \boldsymbol{\sigma} \cdot\left(\begin{array}{l}
1 \\
0 \\
0
\end{array}\right)+\left(\begin{array}{l}
0 \\
1 \\
0
\end{array}\right) \cdot \boldsymbol{\sigma} \cdot\left(\begin{array}{l}
0 \\
1 \\
0
\end{array}\right)+\left(\begin{array}{l}
0 \\
0 \\
1
\end{array}\right) \cdot \boldsymbol{\sigma} \cdot\left(\begin{array}{l}
0 \\
0 \\
1
\end{array}\right)\right) 10^{6} \\
& =\frac{1}{3} \operatorname{Tr}[\boldsymbol{\sigma}] 10^{6}
\end{aligned}
$$


Tr stands for the trace of a matrix. Explicit calculation of this short equation results in the previous expression for the pseudocontact shifts:

$$
\begin{aligned}
\delta_{\mathrm{PCS}}= & \frac{1}{3} \operatorname{Tr}[\boldsymbol{\sigma}] 10^{6} \\
= & \frac{1}{12 \pi r_{I S}^{3}} \operatorname{Tr}\left[3\left(\overrightarrow{e_{r_{I S}}} \otimes \overrightarrow{e_{r_{I S}}}\right) \cdot \chi-\chi\right] 10^{6} \\
= & \frac{1}{12 \pi r_{I S}^{3}}\left(\chi_{x x}\left(3 \sin ^{2}[\theta] \cos ^{2}[\varphi]-1\right)\right. \\
& \left.+\chi_{y y}\left(3 \sin ^{2}[\theta] \sin ^{2}[\varphi]-1\right)+\chi_{z z}\left(3 \cos ^{2}[\theta]-1\right)\right) 10^{6} \\
= & \frac{1}{12 \pi r_{I S}^{3}}\left(\left(3 \cos ^{2}[\theta]-1\right)\left(\chi_{z z}-\frac{\chi_{x x}+\chi_{y y}}{2}\right)+\frac{3}{2}\left(\chi_{x x}-\chi_{y y}\right) \cos [2 \varphi] \sin ^{2}[\theta]\right) 10^{6}
\end{aligned}
$$

The alignment of the molecule due to the anisotropic $\chi$-tensor leads to an additional contribution to the interaction tensor. Therefore, the pseudocontact shift can be expressed as (Eq:37 \& Eq:46):

$$
\begin{aligned}
\delta_{\mathrm{PCS}}= & \left(\frac{1}{3} \operatorname{Tr}[\sigma]+\sigma_{x x} A_{x x}+\sigma_{y y} A_{y y}+\sigma_{z z} A_{z z}\right) 10^{6} \\
= & \left(\frac{1}{3} \operatorname{Tr}[\sigma]+\chi_{x x}\left(3 \sin ^{2}[\theta] \cos ^{2}[\varphi]-1\right) \frac{2}{3} \frac{B_{0}^{2}}{15 \mu_{0} k T}\left(\chi_{x x}-\frac{\chi_{z z}+\chi_{y y}}{2}\right)\right. \\
& +\chi_{y y}\left(3 \sin ^{2}[\theta] \sin ^{2}[\varphi]-1\right) \frac{2}{3} \frac{B_{0}^{2}}{15 \mu_{0} k T}\left(\chi_{y y}-\frac{\chi_{z z}+\chi_{x x}}{2}\right) \\
& \left.+\chi_{z z}\left(3 \cos ^{2}[\theta]-1\right) \frac{2}{3} \frac{B_{0}^{2}}{15 \mu_{0} k T}\left(\chi_{z z}-\frac{\chi_{x x}+\chi_{y y}}{2}\right)\right) 10^{6} \\
= & \frac{1}{12 \pi r_{I S}^{3}}\left(( 3 \operatorname { c o s } ^ { 2 } [ \theta ] - 1 ) \left(\left(\chi_{z z}-\frac{\chi_{x x}+\chi_{y y}}{2}\right)\right.\right. \\
& \left.+\frac{B_{0}^{2}}{15 \mu_{0} k T}\left(\left(\chi_{z z}-\frac{\chi_{x x}+\chi_{y y}}{2}\right)\left(2 \chi_{z z}+\frac{\chi_{x x}+\chi_{y y}}{2}\right)-\frac{3}{4}\left(\chi_{x x}-\chi_{y y}\right)^{2}\right)\right) \\
& \left.+\frac{3}{2}\left(\chi_{x x}-\chi_{y y}\right) \cos [2 \varphi] \sin ^{2}[\theta]\left(1+\frac{B_{0}^{2}}{15 \mu_{0} k T}\left(2 \chi_{x x}+2 \chi_{y y}-\chi_{z z}\right)\right)\right) 10^{6}
\end{aligned}
$$

The influence of the alignment on the pseudocontact shifts is negligible small, which can be seen by calculating the equation for an axial symmetric $\chi$-tensor: $\chi_{z z}=\chi_{\|}, \chi_{x x, y y}=\chi_{\perp}$.

$$
\delta_{\mathrm{PCS}}=\frac{1}{12 \pi r_{I S}^{3}}\left(3 \cos ^{2}[\theta]-1\right)\left(\chi_{\|}-\chi_{\perp}\right)\left(1+\frac{B_{0}^{2}}{15 \mu_{0} k T}\left(2 \chi_{\|}+\chi_{\perp}\right)\right) 10^{6}
$$

For a $900 \mathrm{MHz}$ spectrometer at $298 \mathrm{~K}$ and the $\chi$-tensor of terbium, which has one of the largest known anisotropic $\chi$-tensors with $\chi_{\|}=42.1 \cdot 10^{-32} \mathrm{~m}^{3}$ and $\chi_{\perp}=11.2 \cdot 10^{-32} \mathrm{~m}^{3}$ the latter expression in 
brackets is 1.0055 [Otting2010]. Therefore, even at high field spectrometers the effect of alignment on the pseudocontact shifts is around $0.6 \%$. Furthermore it has been shown that saturation effects of the magnetic susceptibility at high magnetic fields lead to a decrease of the observed shifts [Bertini2002].

\subsection{Residual Chemical Shift Anisotropy - RCSA}

Chemical shifts of nuclei are usually given in ppm. This scale is adjusted to a fix value of a reference substance (e.g. residual solvent signals or 0 for trimethylsilane in proton NMR). The full description of the chemical shift of a nucleus is given by the chemical shift tensor (CS, units in ppm). It is mostly possible to define a reference frame in which the CS-tensor is diagonal.

$$
\sigma_{\mathrm{CS}}=\left(\begin{array}{ccc}
\sigma_{x x} & 0 & 0 \\
0 & \sigma_{y y} & 0 \\
0 & 0 & \sigma_{z z}
\end{array}\right)
$$

In isotropic solution, due to the rapid motion, the anisotropic part is averaged out and the chemical shift is the trace of the chemical shift tensor divided by three:

$$
\sigma_{\mathrm{CS}}^{\text {iso }}=\frac{\operatorname{Tr}\left[\sigma_{\mathrm{CS}}\right]}{3}
$$

In an partially aligned molecule, the anisotropy is not averaged to zero and residual chemical shifts are observed. The anisotropic part of the CS-tensor is traceless and given by:

$$
\sigma_{\mathrm{CS}}^{\text {aniso }}=\left(\begin{array}{ccc}
\sigma_{x x}-\sigma_{\mathrm{CS}}^{\text {iso }} & 0 & 0 \\
0 & \sigma_{y y}-\sigma_{\mathrm{CS}}^{\text {iso }} & 0 \\
0 & 0 & \sigma_{z z}-\sigma_{\mathrm{CS}}^{\text {iso }}
\end{array}\right)
$$

The CSA-tensor can be calculated with density functional theory or back calculated from experimental data with external alignment media. Description of the CSA-tensors are usually given in form of the three diagonal elements, within the reference frame where the tensor is diagonal, and with information about the relation between this frame and the molecular frame. This information, a rotation matrix or Euler angles, allows the transformation of the CSA-tensor into the molecular frame. Once the CSA-tensor is known, the residual chemical shift anisotropy can be calculated. Let $R^{\dagger}$ be the 
rotation matrix of the CSA-tensor to the molecular frame and $R^{\star}$ the rotation of the molecular frame to the alignment tensor frame, then the observed RCSA is:

$$
\sigma_{\mathrm{RCSA}}=\operatorname{Tr}\left[\sigma_{\mathrm{CS}}^{\mathrm{aniso}} \cdot R^{\dagger} \cdot R^{\star} \cdot A\right]
$$

This equation holds for non-paramagnetic aligmnent. The observed changes in the chemical shift of nuclei due to paramagnetic tagging are the sum of the pseudocontact shift and the RCSA:

$$
\delta_{\mathrm{CS}}=\delta_{\mathrm{PCS}}+\sigma_{\mathrm{RCSA}}
$$

\subsection{Paramagnetic Relaxation Enhancement - PRE}

To understand the effects of paramagnetic relaxation enhancement, it is necessary to derive first the standard relaxation equations which are based on the original BPP-theory (Bloembergen, Purcell \& Pound) [Bloembergen1948]. Paramagnetic relaxation is a dipolar effect and because of this, the calculations will be limited to the contribution of dipolar relaxation. As derived before (Eq:12) the dipolar Hamiltonian is given by:

$$
\widehat{\mathscr{H}}_{k l}^{\operatorname{dip}}(t)=-\frac{\mu_{0}}{4 \pi r_{k l}^{3}}\left(3\left(\vec{\mu}_{k} \cdot{\overrightarrow{e_{k l}}}_{r_{k l}}(t)\right)\left(\vec{\mu}_{l} \cdot{\overrightarrow{e_{r_{k l}}}}\right)-\vec{\mu}_{k} \cdot \vec{\mu}_{l}\right)
$$

Relaxation effects are not limited to the part of the magnetic moments which are quantized along an external magnetic field. They have their origin in fluctuating magnetic fields at the nucleus induced by other dipoles (nuclei, electrons, etc.). All three principal components of the magnetic moments have to be considered and the dipolar Hamiltonian has to be written in a different way which will be explained in the next chapter.

\subsubsection{The Dipolar Interaction Hamiltonian Of Two Nuclei}

First, a different notation of the Hamiltonian for two nuclei $(k \& l)$ is derived. The unit vector between the two dipoles is given in spherical coordinates with respect to the external magnetic field, the magnetic moments of the nuclei are written in vector form and $\mathrm{D}_{k l}^{\mathrm{dip}, \mathrm{II}}$ is the dipole coupling 
constant for two like nuclei. The indices are omitted for the angles due to readability reasons.

$$
\begin{array}{ll}
\overrightarrow{e_{r_{k l}}}(t)=\left(\begin{array}{c}
\cos [\varphi] \sin [\theta] \\
\sin [\varphi] \sin [\theta] \\
\cos [\theta]
\end{array}\right) & \mathrm{D}_{k l}^{\mathrm{dip}, \mathrm{II}}=-\frac{\mu_{0}}{4 \pi} \frac{\gamma_{I} \gamma_{k} \hbar^{2}}{r_{k l}^{3}} \\
\vec{\mu}_{k}=\gamma_{k} \hbar \vec{I}_{k}=\gamma_{k} \hbar\left(\begin{array}{c}
I_{k x} \\
I_{k y} \\
I_{k z}
\end{array}\right) & \vec{\mu}_{l}=\gamma_{l} \hbar \vec{I}_{l}=\gamma_{l} \hbar\left(\begin{array}{c}
I_{l x} \\
I_{l y} \\
I_{l z}
\end{array}\right)
\end{array}
$$

With these the dipolar Hamiltonian is given by:

$$
\begin{aligned}
\widehat{\mathscr{H}}_{k l}^{\mathrm{dip}, \mathrm{II}}(t)= & \mathrm{D}_{k l}^{\mathrm{dip}, \mathrm{II}}\left[3 ( I _ { k z } \operatorname { c o s } [ \theta ] + \operatorname { s i n } [ \theta ] ( I _ { k x } \operatorname { c o s } [ \varphi ] + I _ { k y } \operatorname { s i n } [ \varphi ] ) ) \left(I_{l z} \cos [\theta]\right.\right. \\
& \left.\left.+\sin [\theta]\left(I_{l x} \cos [\varphi]+I_{l y} \sin [\varphi]\right)\right)-\vec{I}_{k} \cdot \vec{I}_{l}\right]
\end{aligned}
$$

For the next steps following commutators and expressions are useful.

$$
\begin{aligned}
& {\left[I_{z}, I_{+}\right]=I_{+} \quad\left[I_{-}, I_{z}\right]=I_{-} \quad\left[I_{+}, I_{x}\right]=I_{z} \quad\left[I_{+}, I_{y}\right]=i I_{z} \quad\left[I_{-}, I_{y}\right]=i I_{z}} \\
& {\left[I_{x}, I_{y}\right]=i I_{z} \quad\left[I_{z}, I_{x}\right]=i I_{y} \quad\left[I_{x}, I_{-}\right]=I_{z} \quad\left[I_{y}, I_{z}\right]=i I_{x} \quad\left[I_{+}, I_{-}\right]=2 I_{z}} \\
& I_{x}=\frac{1}{2}\left(I_{+}+I_{-}\right) \quad I_{y}=\frac{1}{2}\left(I_{+}-I_{-}\right) \\
& \cos [\varphi]=\frac{1}{2}(\exp [i \varphi]+\exp [-i \varphi]) \quad \sin [\varphi]=\frac{1}{2}(\exp [i \varphi]-\exp [-i \varphi]) \\
& \exp (i \varphi)=\cos [\varphi]+i \sin [\varphi] \quad 1=\cos ^{2}[\varphi]+\sin ^{2}[\varphi]
\end{aligned}
$$

Reversing the order of two operators in the commutator leads to an inversed sign of the resulting operator. $I_{-}$and $I_{+}$are lowering and raising operators respectively which are defined for calculations with coherence orders but make several derivations much more comfortable [Levitt2001] [Keeler2010]. 
Substituting these into the equation for the dipolar Hamiltonian gives:

$$
\begin{aligned}
\widehat{\mathscr{H}}_{k l}^{\mathrm{dip}, \mathrm{II}}(t)= & \mathrm{D}_{k l}^{\mathrm{dip}, \mathrm{II}}\left[3 \left(I_{k z} \cos [\theta]+\sin [\theta]\left(\frac{1}{2}\left(I_{k+}+I_{k-}\right)\right.\right.\right. \\
& \left.\left.\frac{1}{2}(\exp [i \varphi]+\exp [-i \varphi])+\frac{1}{2 i}\left(I_{k+}-I_{k-}\right) \frac{1}{2 i}(\exp [i \varphi]-\exp [-i \varphi])\right)\right) \\
& \left(I_{l z} \cos [\theta]+\sin [\theta]\left(\frac{1}{2}\left(I_{l+}+I_{l-}\right) \frac{1}{2}(\exp [i \varphi]+\exp [-i \varphi])\right.\right. \\
& \left.\left.+\frac{1}{2 i}\left(I_{l+}-I_{l-}\right) \frac{1}{2 i}(\exp [i \varphi]-\exp [-i \varphi])\right)\right) \\
& \left.-\frac{1}{2} I_{k+} I_{l-}-\frac{1}{2} I_{k-} I_{l+}-\frac{1}{2} I_{k z} I_{l z}\right] \\
= & \mathrm{D}_{k l}^{\mathrm{dip}, \mathrm{II}}\left[I_{k z} I_{l z}\left(3 \cos ^{2}[\theta]-1\right)-\frac{1}{4}\left(I_{k+} I_{l-}+I_{k-} I_{l+}\right)\left(3 \cos ^{2}[\theta]-1\right)\right. \\
& +\frac{3}{2} \cos [\theta] \sin [\theta] \exp [-i \varphi]\left(I_{k+} I_{l z}+I_{k z} I_{l+}\right) \\
& +\frac{3}{2} \cos [\theta] \sin [\theta] \exp [i \varphi]\left(I_{k-} I_{l z}+I_{k z} I_{l-}\right) \\
& \left.+\frac{3}{4} \sin ^{2}[\theta] \exp [-2 i \varphi] I_{k+} I_{l+}+\frac{3}{4} \sin ^{2}[\theta] \exp [2 i \varphi] I_{k-} I_{l-}\right]
\end{aligned}
$$

With following definitions it is possible to write the dipolar Hamiltonian for two nuclei in a very short summation form:

$$
\widehat{\mathscr{H}}_{k l}^{\mathrm{dip}, \mathrm{II}}(t)=\mathrm{D}_{k l}^{\mathrm{dip}, \mathrm{II}} \sum_{-q}^{q} F^{(q)}(t) A^{(q)}
$$

$F^{(q)}(t)$ are called position functions, are time dependent due to random motion in the reference frame of the external magnetic field and $A^{(q)}$ are operator equations with the induced transitions $\Delta m^{(q)}=q$, respectively.

$$
\begin{aligned}
F^{(0)}(t) & =3 \cos ^{2}[\theta]-1 \\
F^{(1)}(t) & =\cos [\theta] \sin [\theta] \exp [-i \varphi] \\
F^{(2)}(t) & =\sin ^{2}[\theta] \exp [-2 i \varphi] \\
\left(F^{(q)}(t)\right)^{\star} & =F^{(-q)}(t) \\
A^{(0)} & =\left(I_{k z} I_{l z}\right)-\frac{1}{4}\left(I_{k+} I_{l-}+I_{k-} I_{l+}\right)=\left(I_{k z} I_{l z}\right)-\frac{1}{2}\left(I_{k x} I_{l x}+I_{k y} I_{l y}\right)
\end{aligned}
$$




$$
\begin{aligned}
A^{(1)} & =\frac{3}{2}\left(I_{k+} I_{l z}+I_{k z} I_{l+}\right) \\
A^{(2)} & =\frac{3}{4} I_{k+} I_{l+} \\
\left(A^{(q)}\right)^{\dagger} & =A^{(-q)}
\end{aligned}
$$

$\star$ stands for the complex conjugate and $\dagger$ stands for the transpose which means in this case just a change between raising and lowering operators. In the literature the Hamiltonian is often written as:

$$
\widehat{\mathscr{H}}_{k l}^{\text {dip,II }}(t)=\mathrm{D}_{k l}^{\text {dip, II }}(A+B+C+D+E+F)
$$

Where the letters stand for different equations with different transitions $\Delta m$.

$$
\begin{aligned}
A & =I_{k z} I_{l z}\left(3 \cos ^{2}[\theta]-1\right) \\
B & =-\frac{1}{4}\left(I_{k+} I_{l-}+I_{k-} I_{l+}\right)\left(3 \cos ^{2}[\theta]-1\right)=-\frac{1}{2}\left(I_{k x} I_{l x}+I_{k y} I_{l y}\right)\left(3 \cos ^{2}[\theta]-1\right) \\
C & =\frac{3}{2}\left(I_{k+} I_{l z}+I_{k z} I_{l+}\right) \cos [\theta] \sin [\theta] \exp [-i \varphi] \\
D & =\frac{3}{2}\left(I_{k-} I_{l z}+I_{k z} I_{l-}\right) \cos [\theta] \sin [\theta] \exp [i \varphi] \\
E & =\frac{3}{4} I_{k+} I_{l+} \sin ^{2}[\theta] \exp [-2 i \varphi] \\
F & =\frac{3}{4} I_{k-} I_{l-} \sin ^{2}[\theta] \exp [-2 i \varphi]
\end{aligned}
$$

In the secular approximation (see Eq14), for identical spins with similar chemical shifts, only the terms $A$ and $B$ are considered. In the heteronuclear case, only $A$ is considered. However, differently from dipolar couplings or pseudocontact shifts induced by the dipolar Hamiltonian, for relaxation all six terms in the dipolar Hamiltonian have to be taken into account.

The largest Hamiltonian at high magnetic fields $\left(B_{0}\right)$ is the isotropic Zeeman interaction Hamiltonian $\widehat{\mathscr{H}}_{0}$ which is time independent:

$$
\widehat{\mathscr{H}_{0}}=\sum_{i} \gamma_{i} B_{0} I_{z_{i}}
$$

The time dependent or time independent Hamiltonians originating from chemical shift anisotropy and dipolar or scalar couplings are small and can therefore treated as perturbations. In the case of time independent Hamiltonians, only the secular terms were taken into account whereas in the case of time 
dependent Hamiltonians, time dependent perturbation theory is applied:

$$
\begin{aligned}
& \widehat{\mathscr{H}}(t)=\widehat{\mathscr{H}_{0}}+\widehat{\mathscr{H}}_{1}(t) \\
& \widehat{\mathscr{H}_{1}}(t)=0
\end{aligned}
$$

The seperation between time dependent and time independent Hamiltonians is done such that the time average of the perturbing Hamiltonian is zero.

It should be noted that the dominating and non-dominating Hamiltonians can be different if the Zeeman interaction is not the dominating interaction. For example, for experiments outside a strong magnetic field, the relaxation theory derived below does not apply.

\subsubsection{The Dipolar Interaction Hamiltonian Of A Nucleus And An Electron}

For the interaction between a nucleus and an electron, which is the origin of paramagnetic relaxation enhancement, the Hamiltonian is derived the same way as above. In following equations, $\mu_{B}$ is the Bohr magneton, $g_{e}$ the electron $g$-factor and $\mathrm{D}^{\text {dip,IS }}$ the coupling constant for a nucleus and an electron.

$$
\begin{aligned}
\vec{\mu}_{I}=\gamma_{I} \hbar \vec{I}=\gamma_{I} \hbar\left(\begin{array}{c}
I_{x} \\
I_{y} \\
I_{z}
\end{array}\right) \quad \vec{\mu}_{S}=-\mu_{B} g_{e} \vec{S}=-\mu_{B} g_{e}\left(\begin{array}{c}
S_{x} \\
S_{y} \\
S_{z}
\end{array}\right) \quad \mathrm{D}^{\mathrm{dip}, \mathrm{IS}}=\frac{\mu_{0}}{4 \pi} \frac{\gamma_{I} \hbar \mu_{B} g_{e}}{r_{I S}^{3}} \\
A^{(0)}=\left(I_{z} S_{z}\right)-\frac{1}{4}\left(I_{+} S_{-}+I_{-} S_{+}\right) \\
A^{(1)}=\frac{3}{2}\left(I_{+} S_{z}+I_{z} S_{+}\right) \\
A^{(2)}=\frac{3}{4} I_{+} S_{+} \\
\widehat{\mathscr{H}}^{\mathrm{dip}, \mathrm{IS}}(t)=\mathrm{D}^{\mathrm{dip}, \mathrm{IS}} \sum_{-q}^{q} F^{(q)}(t) A^{(q)}
\end{aligned}
$$

Even though the anisotropy of the $\boldsymbol{g}_{e^{-}}$-tensor is the origin of the alignment, for relaxation effects which take place on a mikro-to-millisecond timescale, the anisotropy is averaged out due to the fast molecule motion. Consequently, only the isotropic $g_{e}$-value has to be considered for the description of relaxation. First, the complete magnetic moment of the electron (Eq:27) is considered and the 
Curie spin part is calculated later. Therefore, the only change in contrast to the dipolar Hamiltonian for two like nuclei is a change in the coupling constant and the exchange of the operators.

\subsubsection{Lioville - Von Neumann Equation}

The next step towards understanding the relaxation effects is to derive the Liouville - von Neumann equation which describes the interaction of a spin operator with a Hamiltonian. The derivation starts with the time dependent Schrödinger equation which is given by:

$$
\begin{aligned}
i \hbar \frac{\partial}{\partial t}|\Psi(t)\rangle & =\widehat{\mathscr{H}}|\Psi(t)\rangle \\
-i \hbar \frac{\partial}{\partial t}|\Psi(t)\rangle & =\langle\Psi(t)| \widehat{\mathscr{H}}
\end{aligned}
$$

Where $|\Psi\rangle$ and $\langle\Psi|$ stand for the bra and the ket of a wavefunction $\Psi$ respectively. The Hamiltonian can be time dependent or time independent. For simplicity a time independent Hamiltonian is assumed but the implications of a time dependent Hamiltonian will be considered later. A spin density operator is defined in the following way where the overbar indicates a time average and $p_{i}$ is the normalized population of the single states and $\sum_{i} p_{i}=1$.

$$
\rho(t)=\overline{|\Psi(t)\rangle\langle\Psi(t)|}=\sum_{i} p_{i}\left|\Psi_{i}(t)\right\rangle\left\langle\Psi_{i}(t)\right|
$$

The spin density operator describes quantum mechanically a system which consists of several mixed states $\left|\Psi_{i}(t)\right\rangle$. Taking the time derivative and inserting the definitions above, one finally gets the Liouville - von Neumann equation [Cavanagh2007]:

$$
\begin{aligned}
\frac{\partial}{\partial t} \rho(t) & =\sum_{i} p_{i}\left(\frac{\partial}{\partial t}\left|\Psi_{i}(t)\right\rangle\right)\left\langle\Psi_{i}(t)\left|+\sum_{i} p_{i}\right| \Psi_{i}(t)\right\rangle\left(\frac{\partial}{\partial t}\left\langle\Psi_{i}(t)\right|\right) \\
& =-\sum_{i} p_{i}\left(\frac{i}{\hbar} \widehat{\mathscr{H}}\left|\Psi_{i}(t)\right\rangle\right)\left\langle\Psi_{i}(t)\left|+\sum_{i} p_{i}\right| \Psi_{i}(t)\right\rangle\left(\frac{i}{\hbar} \widehat{\mathscr{H}}\left\langle\Psi_{i}(t)\right|\right) \\
& =-\frac{i}{\hbar}\left(\widehat{\mathscr{H}} \sum_{i} p_{i}\left|\Psi_{i}(t)\right\rangle\left\langle\Psi_{i}(t)\left|-\sum_{i} p_{i}\right| \Psi_{i}(t)\right\rangle\left\langle\Psi_{i}(t)\right| \widehat{\mathscr{H}}\right) \\
& =-\frac{i}{\hbar}(\widehat{\mathscr{H}} \rho(t)-\rho(t) \widehat{\mathscr{H}}) \\
& =-\frac{i}{\hbar}[\widehat{\mathscr{H}}, \rho(t)]
\end{aligned}
$$


One important property of this equation is that the time derivative of the density matrix contains the density matrix. When the Hamiltonian is time independent, the straightforward solution for this is the exponential function. The solution is given by $\exp \left[-\frac{i}{\hbar} \widehat{\mathscr{H}} t\right] \rho(0) \exp \left[\frac{i}{\hbar} \widehat{\mathscr{H}} t\right]$ with $\rho(0)$ as a time independent spin density operator.

$$
\begin{aligned}
\frac{\partial}{\partial t} \rho(t) & =\frac{\partial}{\partial t}\left(\exp \left[-\frac{i}{\hbar} \widehat{\mathscr{H}} t\right] \rho(0) \exp \left[\frac{i}{\hbar} \widehat{\mathscr{H} t}\right]\right) \\
& =\frac{\partial}{\partial t}\left(\exp \left[-\frac{i}{\hbar} \widehat{\mathscr{H}} t\right]\right) \rho(0) \exp \left[\frac{i}{\hbar} \widehat{\mathscr{H} t}\right]+\exp \left[-\frac{i}{\hbar} \widehat{\mathscr{H} t}\right] \rho(0) \frac{\partial}{\partial t}\left(\exp \left[\frac{i}{\hbar} \widehat{\mathscr{H} t}\right]\right) \\
& =-\frac{i}{\hbar} \widehat{\mathscr{H}} \exp \left[-\frac{i}{\hbar} \widehat{\mathscr{H}} t\right] \rho(0) \exp \left[\frac{i}{\hbar} \widehat{\mathscr{H} t}\right]+\exp \left[-\frac{i}{\hbar} \widehat{\mathscr{H}} t\right] \rho(0) \frac{i}{\hbar} \widehat{\mathscr{H}} \exp \left[\frac{i}{\hbar} \widehat{\mathscr{H} t}\right] \\
& =-\frac{i}{\hbar}(\widehat{\mathscr{H}} \rho(t)-\rho(t) \widehat{\mathscr{H}}) \\
& =-\frac{i}{\hbar}[\widehat{\mathscr{H}}, \rho(t)]
\end{aligned}
$$

\subsubsection{The Master Equation}

If the Hamiltonian is time dependent, the Liouville - von Neumann equation is still valid but the solution is different. As mentioned above, the Hamiltonian is separated into an time independent and a time dependent Hamiltonian (Eq:57):

$$
\begin{aligned}
\widehat{\mathscr{H}}(t) & =\widehat{\mathscr{H}}_{0}+\widehat{\mathscr{H}}_{1}(t) \\
\frac{\partial}{\partial t} \rho(t) & =-\frac{i}{\hbar}\left[\widehat{\mathscr{H}}_{0}+\widehat{\mathscr{H}}_{1}(t), \rho(t)\right]
\end{aligned}
$$

In order to calculate the spin matrix without the time independent Hamiltonian $\widehat{\mathscr{H}_{0}}$, the matrix and the Hamiltonians are transformed to the interaction frame. This method is called interaction representation and performing calculations in the rotating frame at the Larmor frequency is an example for the interaction representation, which will be used in the following. The transfer of a time dependent or independent operator to the interaction frame of the unperturbed Hamiltonian $\widehat{\mathscr{H}}_{0}$ is given by:

$$
\begin{aligned}
\rho^{*}(t) & =\exp \left[\frac{i}{\hbar} \widehat{\mathscr{H}_{0}} t\right] \rho(t) \exp \left[-\frac{i}{\hbar} \widehat{\mathscr{H}}_{0} t\right] \\
\widehat{\mathscr{H}}_{1}^{*}(t) & =\exp \left[\frac{i}{\hbar} \widehat{\mathscr{H}_{0}} t\right] \widehat{\mathscr{H}}_{1}(t) \exp \left[-\frac{i}{\hbar} \widehat{\mathscr{H}}_{0} t\right]
\end{aligned}
$$


* now indicates an operator in the interaction representation (not to confuse with * which stands for the complex conjugate). The secular parts of a time independent operator stay time independent, while the non-secular parts become time dependent and therefore normally don't contribute to the evolution of the density matrix. Time dependent operators have to be treated differently since they do not directly contribute to the evolution of the density matrix.

The time independent Hamiltonian $\widehat{\mathscr{H}}_{0}$ is unaffected by this transformation as an operator commutes with differentiable functions of itself.

$$
\widehat{\mathscr{H}}_{0}^{*}(t)=\exp \left[\frac{i}{\hbar} \widehat{\mathscr{H}}_{0} t\right] \widehat{\mathscr{H}_{0}} \exp \left[-\frac{i}{\hbar} \widehat{\mathscr{H}}_{0} t\right]=\exp \left[\frac{i}{\hbar} \widehat{\mathscr{H}}_{0} t\right] \exp \left[-\frac{i}{\hbar} \widehat{\mathscr{H}_{0}} t\right] \widehat{\mathscr{H}_{0}}=\widehat{\mathscr{H}}_{0}
$$

Interested only in the perturbing Hamiltonian $\widehat{\mathscr{H}_{1}}(t)$, the density operator is transformed to the unperturbed Hamiltonian frame.

$$
\begin{aligned}
\frac{\partial}{\partial t} \rho^{*}(t)= & \frac{\partial}{\partial t}\left(\exp \left[\frac{i}{\hbar} \widehat{\mathscr{H}_{0}} t\right] \rho(t) \exp \left[-\frac{i}{\hbar} \widehat{\mathscr{H}_{0}} t\right]\right) \\
= & \left(\frac{\partial}{\partial t} \exp \left[\frac{i}{\hbar} \widehat{\mathscr{H}_{0}} t\right]\right) \rho(t) \exp \left[-\frac{i}{\hbar} \widehat{\mathscr{H}_{0}} t\right] \\
& +\exp \left[\frac{i}{\hbar} \widehat{\mathscr{H}_{0}} t\right]\left(\frac{\partial}{\partial t} \rho(t)\right) \exp \left[-\frac{i}{\hbar} \widehat{\mathscr{H}_{0}} t\right] \\
& +\exp \left[\frac{i}{\hbar} \widehat{\mathscr{H}_{0}} t\right] \rho(t)\left(\frac{\partial}{\partial t} \exp \left[-\frac{i}{\hbar} \widehat{\mathscr{H}_{0}} t\right]\right) \\
= & \frac{i}{\hbar} \widehat{\mathscr{H}_{0}} \exp \left[\frac{i}{\hbar} \widehat{\mathscr{H}_{0}} t\right] \rho(t) \exp \left[-\frac{i}{\hbar} \widehat{\mathscr{H}_{0}} t\right] \\
& +\exp \left[\frac{i}{\hbar} \widehat{\mathscr{H}}_{0} t\right]\left(-\frac{i}{\hbar}\left[\widehat{\mathscr{H}_{0}}+\widehat{\mathscr{H}_{1}}(t), \rho(t)\right]\right) \exp \left[-\frac{i}{\hbar} \widehat{\mathscr{H}}_{0} t\right] \\
& -\frac{i}{\hbar} \exp \left[\frac{i}{\hbar} \widehat{\mathscr{H}_{0}} t\right] \rho(t) \widehat{\mathscr{H}}_{0} \exp \left[-\frac{i}{\hbar} \widehat{\mathscr{H}}_{0} t\right] \\
= & \frac{i}{\hbar}\left(\widehat{\mathscr{H}_{0}} \rho^{*}(t)-\rho^{*}(t) \widehat{\mathscr{H}}_{0}\right)-\frac{i}{\hbar}\left[\widehat{\mathscr{H}_{0}}+\widehat{\mathscr{H}}_{1}^{*}(t), \rho^{*}(t)\right] \\
= & \frac{i}{\hbar}\left[\widehat{\mathscr{H}_{0}}, \rho^{*}(t)\right]-\frac{i}{\hbar}\left[\widehat{\mathscr{H}_{0}}, \rho^{*}(t)\right]-\frac{i}{\hbar}\left[\widehat{\mathscr{H}}_{1}^{*}(t), \rho^{*}(t)\right] \\
= & -\frac{i}{\hbar}\left[\widehat{\mathscr{H}}_{1}^{*}(t), \rho^{*}(t)\right]
\end{aligned}
$$

Similarly to above, the time derivative contains the density matrix but the Hamiltonian is also time dependent. To solve this problem, successive substitution up to the second order is used. For this 
technique, first the integration of the Liouville - von Neumann equation is formed and afterwards inserted into itself [Bronstein2008]:

$$
\begin{aligned}
\rho^{*}(t) & =\rho^{*}(0)-\frac{i}{\hbar} \int_{0}^{t}\left[\widehat{\mathscr{H}_{1}^{*}}(t), \rho^{*}(t)\right] \mathrm{d} t \\
& =\rho^{*}(0)-\frac{i}{\hbar} \int_{0}^{t}\left[\widehat{\mathscr{H}_{1}^{*}}(t),\left(\rho^{*}(0)-\frac{i}{\hbar} \int_{0}^{t}\left[\widehat{\mathscr{H}_{1}^{*}}(\hat{t}), \rho^{*}(\hat{t})\right] \mathrm{d} \hat{t}\right)\right] \mathrm{d} t \\
& =\rho^{*}(0)-\frac{i}{\hbar} \int_{0}^{t}\left[\widehat{\mathscr{H}_{1}^{*}}(t), \rho^{*}(0)\right] \mathrm{d} t-\frac{1}{\hbar^{2}} \int_{0}^{t} \int_{0}^{t}\left[\widehat{\mathscr{H}_{1}^{*}}(t),\left[\widehat{\mathscr{H}_{1}^{*}}(\hat{t}), \rho^{*}(\hat{t})\right]\right] \mathrm{d} \hat{t} \mathrm{~d} t
\end{aligned}
$$

To avoid confusion, the variable of the second integral has changed to $t$. Taking the time derivative again results in the following equation:

$$
\frac{\partial}{\partial t} \rho^{*}(t)=-\frac{i}{\hbar}\left[\widehat{\mathscr{H}_{1}^{*}}(t), \rho^{*}(0)\right]-\frac{1}{\hbar^{2}} \int_{0}^{\hat{t}}\left[\widehat{\mathscr{H}_{1}^{*}}(t),\left[\widehat{\mathscr{H}_{1}^{*}}(\hat{t}), \rho^{*}(\hat{t})\right]\right] \mathrm{d} \hat{t}
$$

A complete description of the statistical ensemble of spins is given by an average density operator $\overline{\rho^{*}(t)}$ derived by performing an average of the right-hand-side of the latter equation.

$$
\frac{\partial}{\partial t} \overline{\rho^{*}(t)}=-\frac{i}{\hbar} \overline{\left[\widehat{\mathscr{H}}_{1}^{*}(t), \rho^{*}(0)\right]}-\frac{1}{\hbar^{2}} \int_{0}^{t} \overline{\left[\widehat{\mathscr{H}}_{1}^{*}(t),\left[\widehat{\mathscr{H}_{1}^{*}}(\hat{t}), \rho^{*}(\hat{t})\right]\right]} \mathrm{d} \dot{t}
$$

Changing the variable of the integral according to $t=t+\tau$ this equation becomes:

$$
\frac{\partial}{\partial t} \overline{\rho^{*}(t)}=-\frac{i}{\hbar} \overline{\left[\widehat{\mathscr{H}}_{1}^{*}(t), \rho^{*}(0)\right]}-\frac{1}{\hbar^{2}} \int_{0}^{t} \overline{\left[\widehat{\mathscr{H}}_{1}^{*}(t),\left[\widehat{\mathscr{H}}_{1}^{*}(t+\tau), \rho^{*}(t+\tau)\right]\right]} \mathrm{d} \tau
$$

It will be shown later that the correlation between the two Hamiltonians in the integral of Eq:66 can be described by an exponential decay with a correlational time constant $\tau_{c}$ which is in the picoseconds to nanoseconds range for molecules in solution. At this point four assumptions simplify the further calculation.

1. Successive substitution up to second order is sufficient. The next iteration would add a term which depends on two times the exponential decay and thus is negligibly small.

2. Performing an ensemble average removes the time dependency from the term $\overline{\left[\widehat{\mathscr{H}}_{1}^{*}(t), \rho^{*}(0)\right]}$ because of the large amount of independent systems in the ensemble. Time independent effects are included in the time independent $\widehat{\mathscr{H}_{0}}$ by construction and thus $\overline{\left[\widehat{\mathscr{H}}_{1}^{*}(t), \rho^{*}(0)\right]}$ vanishes.

3. Remarkable contribution to the value of the integral is limited to the range $0 \leq \tau \leq 3 \tau_{c}$. During this period the change of the average density operator $\overline{\rho^{*}(t+\tau)}$ and the correlation to the Hamiltonians is negligible. The operator can then be averaged independently and for the averaged term it is valid to replace it with $\overline{\rho^{*}(t)}$. 
4. For the same reason the error by changing the upper limit of the integral to $\infty$ is negligible small.

With these assumptions the master equation is given by:

$$
\frac{\partial}{\partial t} \overline{\rho^{*}(t)}=-\frac{1}{\hbar^{2}} \int_{0}^{\infty} \overline{\left[\widehat{\mathscr{H}}_{1}^{*}(t),\left[\widehat{\mathscr{H}_{1}^{*}}(t+\tau), \rho^{*}(t)\right]\right]} \mathrm{d} \tau
$$

\subsubsection{Relaxation Of Two Like Spins}

In the dipolar Hamiltonian for two like spins (Eq:54), the position functions $F^{(q)}(t)$ are time dependent and the operator equations $A^{(q)}$ are time independent. Since the dipolar Hamiltonian has to be expressed in the interaction frame in the master equation, the operator equations have to be transformed into the interaction representation:

$$
\begin{aligned}
A^{(q)}(t) & =\exp \left[i \widehat{\mathscr{H}}_{0} t\right] A^{(q)} \exp \left[-i \widehat{\mathscr{H}}_{0} t\right]=\sum_{q, p} A_{(p)}^{(q)} \exp \left[-i \omega_{(p)}^{(q)} t\right] \\
A^{(-q)}(t) & =\exp \left[i \widehat{\mathscr{H}}_{0} t\right] A^{(-q)} \exp \left[-i \widehat{\mathscr{H}}_{0} t\right]=\sum_{q, p} A_{(p)}^{(-q)} \exp \left[-i \omega_{(p)}^{(-q)} t\right]
\end{aligned}
$$

$\omega$ are the Larmor frequencies of the corresponding terms in the operator equations. The index $p$ stands for the different terms in the operator equations because each of these terms has a different Larmor frequency $(p=1,2,3 \& q=-2,-1,0,1,2)$.

A raising operator results in a positive and a lowering operator in a negative frequency whereas a $z$-operator results in no frequency, which can be seen with a part of $A^{(1)}$, i.e. $I_{+} S_{z}$ inserted into the Liouville - von Neumann equation with $\widehat{\mathscr{H}_{0}}=\hbar\left(\omega_{I} I_{z}+\omega_{S} S_{z}\right)$ :

$$
\begin{gathered}
\frac{\partial}{\partial t}\left(I_{+} S_{z}(t)\right)=-\frac{i}{\hbar}\left[\widehat{\mathscr{H}_{0}}, I_{+} S_{z}\right]=-i\left[\omega_{I} I_{z}+\omega_{S} S_{z}, I_{+} S_{z}\right]=-i \omega_{I} I_{+} S_{z} \\
I_{+} S_{z}(t)=\exp \left[-i \omega_{I} t\right] I_{+} S_{z}
\end{gathered}
$$

The dipolar Hamiltonian in the interaction representation now reads:

$$
\begin{aligned}
\widehat{\mathscr{H}}_{k l}^{\mathrm{dip}, \mathrm{II} *}(t) & =\mathrm{D}_{k l}^{\mathrm{dip}, \mathrm{II}} \sum_{q, p} F^{(q)}(t) A_{(p)}^{(q)} \exp \left[-i \omega_{(p)}^{(q)} t\right] \\
\widehat{\mathscr{H}}_{k l}^{\mathrm{dip}, \mathrm{II} *}(t+\tau) & =\mathrm{D}_{k l}^{\mathrm{dip}, \mathrm{II}} \sum_{q, p} F^{(q)}(t+\tau) A_{(p)}^{(q)} \exp \left[-i \omega_{(p)}^{(q)}(t+\tau)\right]
\end{aligned}
$$


Inserting these equations into the master equation $(\mathrm{Eq}: 67)$ and recalling that every time dependent term is averaged gives:

$$
\begin{aligned}
& \frac{\partial}{\partial t} \overline{\rho^{*}(t)}=-\frac{1}{\hbar^{2}} \int_{0}^{t} \overline{\left[\widehat{\mathscr{H}}_{1}^{*}(t),\left[\widehat{\mathscr{H}}_{1}^{*}(t+\tau), \rho^{*}(t+\tau)\right]\right]} \mathrm{d} \tau \\
& =-\frac{\left(\mathrm{D}_{k l}^{\mathrm{dip}, \mathrm{II}}\right)^{2}}{\hbar^{2}} \sum_{q, \dot{q}, p, \dot{p}}\left[A_{(\dot{p})}^{(\dot{q})},\left[A_{(p)}^{(q)}, \overline{\rho^{*}(t)}\right]\right] \\
& \int_{0}^{\infty} \overline{F^{(q)}(t) F^{(\hat{q})}(t+\tau)} \overline{\exp \left[i \omega_{(p)}^{(q)} t\right] \exp \left[i \omega_{(\tilde{p})}^{(\hat{q})}(t+\tau)\right]} \mathrm{d} \tau \\
& =-\frac{\left(\mathrm{D}_{k l}^{\mathrm{dip}, \mathrm{II}}\right)^{2}}{\hbar^{2}} \sum_{q, \hat{q}, p, \dot{p}}\left[A_{(\hat{p})}^{(\hat{q})},\left[A_{(p)}^{(q)}, \overline{\rho^{*}(t)}\right]\right] \overline{\exp \left[i\left(\omega_{(p)}^{(q)}+\omega_{(\dot{p})}^{(\hat{q})}\right) t\right]} \\
& \int_{0}^{\infty} \overline{F^{(q)}(t) F^{(\hat{q})}(t+\tau)} \exp \left[i \omega_{(\dot{p})}^{(\hat{q})} \tau\right] \mathrm{d} \tau
\end{aligned}
$$

With $\omega_{(p)}^{(q)}=-\omega_{(p)}^{(-q)}$ and the $q$ and $\not \dot{p}$ indices are introduced because after a given time $\tau$ the operator and position functions aren't the same anymore. Each exponential with rapidly varying arguments averages approximately to zero. The only terms to consider are the terms with $q=-q$.

$$
\begin{aligned}
& \dot{q}=-q \longrightarrow \exp \left[i\left(\omega_{(p)}^{(q)}+\omega_{(\dot{p})}^{(\dot{q})}\right) t\right]=1 \\
& \dot{q} \neq-q \longrightarrow \exp \left[i\left(\omega_{(p)}^{(q)}+\omega_{(\dot{p})}^{(\dot{q})}\right) t\right] \simeq 0 \\
& \int_{0}^{\infty} \overline{F^{(q)}(t) F^{(\hat{q})}(t+\tau)} \exp \left[i \omega_{(\hat{p})}^{(\hat{q})} \tau\right] \mathrm{d} \tau=\int_{0}^{\infty} \overline{F^{(q)}(t) F^{(-q)}(t+\tau)} \exp \left[i \omega_{(p)}^{(-q)} \tau\right] \mathrm{d} \tau \\
& =\int_{0}^{\infty} \overline{F^{(q)}(t) F^{(q)^{\star}}(t+\tau)} \exp \left[-i \omega_{(p)}^{(q)} \tau\right] \mathrm{d} \tau
\end{aligned}
$$

* stands for the complex conjugate. The real part of this term is called power spectral density whereas the imaginary part is the dynamic frequency shift [Werbelow1979].

$$
\begin{gathered}
\int_{0}^{\infty} \overline{F^{(q)}(t) F^{(q)^{\star}}(t+\tau)} \exp \left[-i \omega_{(p)}^{(q)} \tau\right] \mathrm{d} \tau \\
=\operatorname{Re} \int_{0}^{\infty} \overline{F^{(q)}(t) F^{(q)^{\star}}(t+\tau)} \exp \left[-i \omega_{(p)}^{(q)} \tau\right] \mathrm{d} \tau+\operatorname{Im} \int_{0}^{\infty} \overline{F^{(q)}(t) F^{(q)^{\star}}(t+\tau)} \exp \left[-i \omega_{(p)}^{(q)} \tau\right] \mathrm{d} \tau
\end{gathered}
$$

The power spectral density is defined as:

$$
J_{(p)}^{(q)}\left(\omega_{(p)}^{(q)}\right)=\operatorname{Re} \int_{0}^{\infty} \overline{F^{(q)}(t) F^{(q)^{\star}}(t+\tau)} \exp \left[-i \omega_{(p)}^{(q)} \tau\right] \mathrm{d} \tau
$$


Because it is an even term following relations hold:

$$
J_{(p)}^{(q)}\left(\omega_{(p)}^{(q)}\right)=J_{(p)}^{(-q)}\left(\omega_{(p)}^{(q)}\right)=J_{(p)}^{(-q)}\left(-\omega_{(p)}^{(q)}\right)=J_{(p)}^{(-q)}\left(\omega_{(p)}^{(-q)}\right)
$$

The dynamic frequency shift is given by:

$$
K_{(p)}^{(q)}\left(\omega_{(p)}^{(q)}\right)=\operatorname{Im} \int_{0}^{\infty} \overline{F^{(q)}(t) F^{(q)^{\star}}(t+\tau)} \exp \left[-i \omega_{(p)}^{(q)} \tau\right] \mathrm{d} \tau
$$

The master equation in its operator form now reads:

$$
\frac{\partial}{\partial t} \overline{\rho^{*}(t)}=-\frac{\left(\mathrm{D}_{k l}^{\mathrm{dip}, \mathrm{II}}\right)^{2}}{\hbar^{2}} \sum_{q, p}\left[A_{(p)}^{(-q)},\left[A_{(p)}^{(q)}, \overline{\rho^{*}(t)}\right]\right]\left(J_{(p)}^{(q)}\left(\omega_{(p)}^{(q)}\right)+i K_{(p)}^{(q)}\left(\omega_{(p)}^{(q)}\right)\right)
$$

The dynamic frequency shift is imaginary and because of this, it is a time independent contribution and can be included into the unperturbed Hamiltonian which reduces the equation of motion for the spin density matrix to:

$$
\frac{\partial}{\partial t} \overline{\rho^{*}(t)}=-\frac{\left(\mathrm{D}_{k l}^{\mathrm{dip}, \mathrm{II}}\right)^{2}}{\hbar^{2}} \sum_{q, p}\left[A_{(p)}^{(-q)},\left[A_{(p)}^{(q)}, \overline{\rho^{*}(t)}\right]\right] J_{(p)}^{(q)}\left(\omega_{(p)}^{(q)}\right)
$$

The next step towards the observable behavior of the system in the interaction representation $O^{*}(t)$ is to calculate the expectation value $\langle\hat{O}\rangle^{*}$ of the corresponding operator $\hat{O}$ acting on the system. This is given by taking the trace of the product of the density matrix with the operator [Hubbard1961]:

$$
\begin{gathered}
O^{*}(t)=\langle\hat{O}\rangle^{*}=\operatorname{Tr}\left[\overline{\rho^{*}(t) \hat{O}}\right] \\
\frac{\partial}{\partial t} O^{*}(t)=\operatorname{Tr}\left[-\frac{\left(\mathrm{D}_{k l}^{\mathrm{dip}, \mathrm{II}}\right)^{2}}{\hbar^{2}} \sum_{q, p}\left[A_{(p)}^{(-q)},\left[A_{(p)}^{(q)}, \overline{\rho^{*}(t)}\right]\right] J_{(p)}^{(q)}\left(\omega_{(p)}^{(q)}\right) \hat{O}\right]
\end{gathered}
$$

The angle brackets indicate the expectation value of the corresponding operator. Traces of products of operators are invariant under cyclic permutations:

$$
\operatorname{Tr}[A,[B, C]]=\operatorname{Tr}[B,[C, A]]=\operatorname{Tr}[C,[A, B]]=\operatorname{Tr}[A,[B, C]]=\operatorname{Tr}[C,[A, B]]=\operatorname{Tr}[B,[C, A]]
$$

Repeated usage of this relation leads to following equation:

$$
\frac{\partial}{\partial t} O^{*}(t)=\operatorname{Tr}\left[-\frac{\left(\mathrm{D}_{k l}^{\mathrm{dip}, \mathrm{II}}\right)^{2}}{\hbar^{2}} \sum_{q, p}\left[A_{(p)}^{(-q)},\left[A_{(p)}^{(q)}, \hat{O}\right]\right] J_{(p)}^{(q)}\left(\omega_{(p)}^{(q)}\right) \overline{\rho^{*}(t)}\right]
$$


With the definition of a new operator $\hat{B}$, the equation for the observable behavior is given below:

$$
\begin{gathered}
\hat{B}=-\frac{\left(\mathrm{D}_{k l}^{\mathrm{dip}, \mathrm{II}}\right)^{2}}{\hbar^{2}} \sum_{q, p}\left[A_{(p)}^{(-q)},\left[A_{(p)}^{(q)}, \hat{O}\right]\right] J_{(p)}^{(q)}\left(\omega_{(p)}^{(q)}\right) \\
\frac{\partial}{\partial t} O^{*}(t)=\operatorname{Tr}\left[\hat{B} \widehat{\rho^{*}(t)}\right]=\langle\hat{B}\rangle
\end{gathered}
$$

Thus, to calculate the effects of an operator, it is neither necessary to know the spin density operator nor to calculate the time evolution of it. The only thing to calculate is the operator $\hat{B}$.

Considering first longitudinal relaxation for two like spins, the operator is $I_{k z}+I_{l z}$ and the results are obtained by calculating the operator $\hat{B}$ given by:

$$
\hat{B}=-\frac{\left(\mathrm{D}_{k l}^{\mathrm{dip}, \mathrm{II}}\right)^{2}}{\hbar^{2}} \sum_{q, p}\left[A_{(p)}^{(-q)},\left[A_{(p)}^{(q)}, I_{k z}+I_{l z}\right]\right] J_{(p)}^{(q)}\left(\omega_{(p)}^{(q)}\right)
$$

Explicit calculations lead to following results:

$$
\begin{aligned}
\sum_{p}\left[A_{(p)}^{(0)},\left[A_{(p)}^{(0)}, I_{k z}+I_{l z}\right]\right] J_{(p)}^{(0)}\left(\omega_{(p)}^{(0)}\right) & =0 \\
\sum_{p}\left[A_{(p)}^{(-1)},\left[A_{(p)}^{(1)}, I_{k z}+I_{l z}\right]\right] J_{(p)}^{(1)}\left(\omega_{(p)}^{(1)}\right) & =\sum_{p}\left[A_{(p)}^{(1)},\left[A_{(p)}^{(-1)}, I_{k z}+I_{l z}\right]\right] J_{(p)}^{(1)}\left(\omega_{(p)}^{(1)}\right) \\
& =\frac{9}{8}\left(I_{k z}+I_{l z}\right) J^{(1)}\left(\omega_{I}\right) \\
\sum_{p}\left[A_{(p)}^{(-2)},\left[A_{(p)}^{(2)}, I_{k z}+I_{l z}\right]\right] J_{(p)}^{(2)}\left(\omega_{(p)}^{(2)}\right) & =\sum_{p}\left[A_{(p)}^{(2)},\left[A_{(p)}^{(-2)}, I_{k z}+I_{l z}\right]\right] J_{(p)}^{(2)}\left(\omega_{(p)}^{(2)}\right) \\
& =\frac{9}{8}\left(I_{k z}+I_{l z}\right) J^{(2)}\left(2 \omega_{I}\right)
\end{aligned}
$$

The relaxation equation for longitudinal relaxation now reads:

$$
\frac{\partial}{\partial t}\left(I_{k z}+I_{l z}\right)=-\frac{9}{4} \frac{\left(\mathrm{D}_{k l}^{\mathrm{dip}, I I}\right)^{2}}{\hbar^{2}}\left(I_{k z}+I_{l z}\right)\left(J^{(1)}\left(\omega_{I}\right)+J^{(2)}\left(2 \omega_{I}\right)\right)
$$

$J^{(0)}\left(\omega_{I_{k}}-\omega_{I_{l}}\right)=0$ and $J^{(2)}\left(\omega_{I_{k}}+\omega_{I_{l}}\right)=J^{(2)}\left(2 \omega_{I}\right)$ because of the identity of the spins. Therefore, the dipolar longitudinal relaxation rate $R_{1}=1 / T_{1}$ is given by:

$$
\begin{aligned}
\frac{1}{T_{1}} & =\frac{9}{4} \frac{\left(\mathrm{D}_{k l}^{\mathrm{dip}, \mathrm{II}}\right)^{2}}{\hbar^{2}}\left(J^{(1)}\left(\omega_{I}\right)+J^{(2)}\left(2 \omega_{I}\right)\right) \\
& =\frac{9}{4}\left(\frac{\mu_{0}}{4 \pi}\right)^{2} \frac{\gamma_{k}^{2} \gamma_{l}^{2} \hbar^{2}}{r_{k l}^{6}}\left(J^{(1)}\left(\omega_{I}\right)+J^{(2)}\left(2 \omega_{I}\right)\right)
\end{aligned}
$$


Expressions for the different spectral densities will be calculated in a later section (2.6.8). For the transverse relaxation time the operator is $I_{k x}+I_{l x}$.

$$
\begin{aligned}
& \sum_{p}\left[A_{(p)}^{(0)},\left[A_{(p)}^{(0)}, I_{k x}+I_{l x}\right]\right] J_{(p)}^{(0)}\left(\omega_{(p)}^{(0)}\right)=\frac{9}{16}\left(I_{k x}+I_{l x}\right) J^{(0)}(0) \\
& \sum_{p}\left[A_{(p)}^{(-1)},\left[A_{(p)}^{(1)}, I_{k x}+I_{l x}\right]\right] J_{(p)}^{(1)}\left(\omega_{(p)}^{(1)}\right)+\sum_{p}\left[A_{(p)}^{(1)},\left[A_{(p)}^{(-1)}, I_{k x}+I_{l x}\right]\right] J_{(p)}^{(-1)}\left(\omega_{(p)}^{(-1)}\right) \\
& =\frac{135}{24}\left(I_{k x}+I_{l x}\right) J^{(1)}\left(\omega_{I}\right) \\
& \sum_{p}\left[A_{(p)}^{(-2)},\left[A_{(p)}^{(2)}, I_{k x}+I_{l x}\right]\right] J_{(p)}^{(2)}\left(\omega_{(p)}^{(2)}\right)+\sum_{p}\left[A_{(p)}^{(2)},\left[A_{(p)}^{(-2)}, I_{k x}+I_{l x}\right]\right] J_{(p)}^{(-2)}\left(\omega_{(p)}^{(-2)}\right) \\
& =\frac{9}{16}\left(I_{k x}+I_{l x}\right) J^{(2)}\left(2 \omega_{I}\right)
\end{aligned}
$$

This leads to the following equations for the relaxation and the transverse relaxation rate $R_{2}=1 / T_{2}$ :

$$
\begin{aligned}
\frac{\partial}{\partial t}\left(I_{k x}+I_{l x}\right) & =-\frac{3}{4} \frac{\left(\mathrm{D}_{k l}^{\mathrm{dip}, \mathrm{II}}\right)^{2}}{\hbar^{2}}\left(I_{k x}+I_{l x}\right)\left(\frac{3}{4} J^{(0)}(0)+\frac{45}{6} J^{(1)}\left(\omega_{I}\right)+\frac{3}{4} J^{(2)}\left(2 \omega_{I}\right)\right) \\
\frac{1}{T_{2}} & =\frac{3}{4} \frac{\left(\mathrm{D}_{k l}^{\mathrm{dip}, \mathrm{II}}\right)^{2}}{\hbar^{2}}\left(\frac{3}{4} J^{(0)}(0)+\frac{45}{6} J^{(1)}\left(\omega_{I}\right)+\frac{3}{4} J^{(2)}\left(2 \omega_{I}\right)\right) \\
& =\frac{3}{4}\left(\frac{\mu_{0}}{4 \pi}\right)^{2} \frac{\gamma_{k}^{2} \gamma_{l}^{2} \hbar^{2}}{r_{k l}^{6}}\left(\frac{3}{4} J^{(0)}(0)+\frac{45}{6} J^{(1)}\left(\omega_{I}\right)+\frac{3}{4} J^{(2)}\left(2 \omega_{I}\right)\right)
\end{aligned}
$$

\subsubsection{Relaxation Of Unlike Spins - Nucleus And Electron}

The dipolar Hamiltonian for two unlike spins is of the same form as for two like spins with another coupling constant $\mathrm{D}^{\text {dip,IS }}$ and another set of operator equations given above.

$$
\widehat{\mathscr{H}}^{\mathrm{dip}, \mathrm{IS}}(t)=\mathrm{D}^{\mathrm{dip}, \mathrm{IS}} \sum_{-q}^{q} F^{(q)}(t) A^{(q)}
$$

The Larmor frequencies of electrons are much higher than of nuclei and therefore each spectral density dependent on the Larmor frequency of the electron is assumed to be zero for relaxation of nuclei. A proof of this is given when calculating the spectral densities. For the longitudinal relaxation, the operators $I_{z}$ and $S_{z}$ can be observed independently. The results for these two are identical, except for a change of $I$ and $S$, and only the results for nuclei are shown.

$$
\hat{B}=-\frac{\left(\mathrm{D}^{\mathrm{dip}, I S}\right)^{2}}{\hbar^{2}} \sum_{q, p}\left[A_{(p)}^{(-q)},\left[A_{(p)}^{(q)}, I_{z}\right]\right] J_{(p)}^{(q)}\left(\omega_{(p)}^{(q)}\right)
$$


Longitudinal relaxation:

$$
\begin{gathered}
\sum_{q, p}\left[A_{(p)}^{(-q)},\left[A_{(p)}^{(q)}, I_{z}\right]\right] J_{(p)}^{(q)}\left(\omega_{(p)}^{(q)}\right)=\frac{9}{4} I_{z} J^{(1)}\left(\omega_{I}\right) \\
\frac{1}{T_{1}}=\frac{9}{4} \frac{\left(\mathrm{D}^{\mathrm{dip}, I S}\right)^{2}}{\hbar^{2}} J^{(1)}\left(\omega_{I}\right)=\frac{9}{4}\left(\frac{\mu_{0}}{4 \pi}\right)^{2} \frac{\gamma_{I}^{2} g_{e}^{2} \mu_{B}^{2}}{r_{I S}^{6}} J^{(1)}\left(\omega_{I}\right)
\end{gathered}
$$

Transverse relaxation:

$$
\begin{aligned}
\sum_{q, p}\left[A_{(p)}^{(-q)},\right. & {\left.\left[A_{(p)}^{(q)}, I_{x}\right]\right] J_{(p)}^{(q)}\left(\omega_{(p)}^{(q)}\right)=\frac{3}{12} I_{x} J^{(0)}(0)+\frac{9}{8} I_{x} J^{(1)}\left(\omega_{I}\right) } \\
\frac{1}{T_{2}} & =\frac{3}{4} \frac{\left(\mathrm{D}^{\mathrm{dip}, I S}\right)^{2}}{\hbar^{2}}\left(\frac{1}{3} J^{(0)}(0)+\frac{3}{2} J^{(1)}\left(\omega_{I}\right)\right) \\
& =\frac{3}{4}\left(\frac{\mu_{0}}{4 \pi}\right)^{2} \frac{\gamma_{I}^{2} g_{e}^{2} \mu_{B}^{2}}{r_{I S}^{6}}\left(\frac{1}{3} J^{(0)}(0)+\frac{3}{2} J^{(1)}\left(\omega_{I}\right)\right)
\end{aligned}
$$

\subsubsection{Relaxation Of Two Unlike Nuclei}

For completeness, the longitudinal relaxation rates for two unlike nuclei are given. In this case the spectral densities dependent on the sum and the difference of the Larmor frequencies have to be considered. The index $S$ in the next equations stands for a second nucleus with a different Larmor frequency.

$$
\begin{aligned}
\sum_{q, p}\left[A_{(p)}^{(-q)},\left[A_{(p)}^{(q)}, I_{z}\right]\right] J_{(p)}^{(q)}\left(\omega_{(p)}^{(q)}\right)= & \frac{1}{8}\left(I_{z}-S_{z}\right) J^{(0)}\left(\omega_{I}-\omega_{S}\right) \\
& +\frac{9}{4} I_{z} J^{(1)}\left(\omega_{I}\right) \\
& +\frac{9}{8} I_{Z} J^{(2)}\left(\omega_{I}+\omega_{S}\right) \\
& +\frac{9}{8} S_{z} J^{(2)}\left(\omega_{I}+\omega_{S}\right)
\end{aligned}
$$

Similar equations are obtained for the $S$-spin by exchanging $I$ and $S$ in the above equations. The two terms which depend on the spin state of the $S$-spin correspond to cross relaxation $\left(\sigma_{I S}\right)$ between the two spins whereas the other three terms correspond to auto relaxation of the $I$-spin $\left(\rho_{I}\right)$.

$$
\begin{gathered}
\sigma_{I S}=\frac{1}{T_{1}^{c r}}=\left(\frac{\mu_{0}}{4 \pi}\right)^{2} \frac{\gamma_{I}^{2} \gamma_{S}^{2} \hbar^{2}}{r_{I S}^{6}}\left(-\frac{1}{8} J^{(0)}\left(\omega_{I}-\omega_{S}\right)+\frac{9}{8} J^{(2)}\left(\omega_{I}+\omega_{S}\right)\right) \\
\rho_{I}=\frac{1}{T_{1}^{\text {auto }}}=\left(\frac{\mu_{0}}{4 \pi}\right)^{2} \frac{\gamma_{I}^{2} \gamma_{S}^{2} \hbar^{2}}{r_{I S}^{6}}\left(\frac{1}{8} J^{(0)}\left(\omega_{I}-\omega_{S}\right)+\frac{9}{4} J^{(1)}\left(\omega_{I}\right)+\frac{9}{8} J^{(2)}\left(\omega_{I}+\omega_{S}\right)\right)
\end{gathered}
$$


The simple relaxation matrix for two nuclei spins $(I \& S)$ is given by [Cavanagh2007]:

$$
\frac{\partial}{\partial t}\left(\begin{array}{l}
I_{z} \\
S_{z}
\end{array}\right)=-\left(\begin{array}{ll}
\rho_{I} & \sigma_{I S} \\
\sigma_{I S} & \rho_{S}
\end{array}\right)\left(\begin{array}{c}
\Delta I_{z} \\
\Delta S_{z}
\end{array}\right)
$$

$\Delta I_{z}$ and $\Delta S_{z}$ are the deviations of the magnetizations from the equlibrium values. $\rho$ indicates auto relaxation rates and $\sigma$ dipole-dipole cross relaxation rates.

\subsubsection{Calculation Of The Spectral Densities}

The next step to get a complete expression for the relaxation rates is to find the spectral densities:

$$
J_{(p)}^{(q)}\left(\omega_{(p)}^{(q)}\right)=\operatorname{Re} \int_{0}^{\infty} \overline{F^{(q)}(t) F^{(q)^{\star}}(t+\tau)} \exp \left[-i \omega_{(p)}^{(q)} \tau\right] \mathrm{d} \tau
$$

The term $\overline{\left(F^{(q)}(t) F^{(q)^{\star}}(t+\tau)\right)}$ is called correlation function $G^{(q)}(\tau)$ and is an indicator for the similarity of two position functions at different times. The correlation function can be written in the following probability form [Hubbard1958]:

$$
G^{(q)}(\tau)=\frac{1}{4 \pi} \iint F^{(q)}(\Omega) F^{(q)^{\star}}\left(\Omega_{0}\right) P\left(\Omega, \Omega_{0}, \tau\right) \mathrm{d} \Omega \mathrm{d} \Omega_{0}
$$

With this, the expression for the power spectral density is:

$$
J_{(p)}^{(q)}\left(\omega_{(p)}^{(q)}\right)=\operatorname{Re} \int_{0}^{\infty} G^{(q)}(\tau) \exp \left[-i \omega_{(p)}^{(q)} \tau\right] \mathrm{d} \tau
$$

$\Omega$ and $\Omega_{0}$ stand for two sets of spherical coordinates, $P\left(\Omega, \Omega_{0}, \tau\right)$ is the probability density function, which describes the likelihood of the two position functions dependent on $\tau$. Furthermore the time average was replaced by an average over the spherical coordinates, where $\int \mathrm{d} \Omega=\iint_{0,0}^{2 \pi, \pi} \sin [\theta] \mathrm{d} \theta \mathrm{d} \varphi$ which normalizes the term. The factor $1 / 4 \pi$ is the probability to find the system in any arbitrary state and thus constant because only the difference between two states separated by $\tau$ is considered.

The following derivation follows the description given in Principles of Nuclear Magnetism [Abragam1961]. The process which alters a position function with time is the rotational diffusion. Fick's second law describes translational diffusion and is shown in Eq:88(left). $c$ is the concentration, $D$ the diffusion coefficient $\left(\mathrm{m}^{2} / \mathrm{s}\right)$ and $\nabla$ the nabla operator. The right equation is valid for a constant diffusion coefficient D.

$$
\frac{\partial}{\partial t} c=\nabla(D \nabla c) \quad \frac{\partial}{\partial t} c=\mathrm{D}^{2} c
$$


Assuming that the diffusion is described by diffusion on an unit sphere ( $r=1=$ const) and expanding the nabla operator in spherical coordinates lead to the rotational form of Fick's second law which describes the rotational displacement of the probability density function $P\left(\Omega, \Omega_{0}, \tau\right)$ :

$$
\frac{\partial}{\partial \tau} P\left(\Omega, \Omega_{0}, \tau\right)=\mathrm{D}\left(\frac{1}{\sin [\theta]} \frac{\partial}{\partial \theta}\left(\sin [\theta] \frac{\partial}{\partial \theta}\right)+\frac{1}{\sin ^{2}[\theta]} \frac{\partial^{2}}{\partial \varphi^{2}}\right) P\left(\Omega, \Omega_{0}, \tau\right)
$$

The diffusion coefficient has now the unit of $1 / \mathrm{s}$. One solution to this equation is obtained by expanding the probability density function in terms of spherical harmonics $Y_{l}^{m}$ which have the following property:

$$
\begin{gathered}
\frac{1}{\sin [\theta]} \frac{\partial}{\partial \theta}\left(\sin [\theta] \frac{\partial Y_{l}^{m}}{\partial \theta}\right)+\frac{1}{\sin ^{2}[\theta]} \frac{\partial^{2} Y_{l}^{m}}{\partial \varphi^{2}}=-l(l+1) Y_{l}^{m} \\
P\left(\Omega, \Omega_{0}, \tau\right)=\sum_{l, m} c_{l}^{m}\left(\Omega_{0}, \tau\right) Y_{l}^{m}(\Omega)
\end{gathered}
$$

By substituting these relations into Eq 89 , the following equation is obtained:

$$
\frac{\partial}{\partial \tau} c_{l}^{m}\left(\Omega_{0}, \tau\right)=-l(l+1) \mathrm{D} c_{l}^{m}\left(\Omega_{0}, \tau\right)
$$

Therefore, $c_{l}^{m}\left(\Omega_{0}, \tau\right)=c_{l}^{m}\left(\Omega_{0}\right) \exp [-\tau \mathrm{D} l(l+1)]$ and by substituting $\mathrm{D} l(l+1)$ with $\tau_{r}^{-1}$ the solution of the partial differential equation is:

$$
P\left(\Omega, \Omega_{0}, \tau\right)=\sum_{l, m} c_{l}^{m}\left(\Omega_{0}\right) Y_{l}^{m}(\Omega) \exp \left[-\frac{\tau}{\tau_{r}}\right]
$$

The initial probability density function $P\left(\Omega_{0}, \Omega\right)$ can be described by two sets of complete spherical harmonics:

$$
P\left(\Omega_{0}, \Omega\right)=\sum_{l, m} Y_{l}^{m \star}\left(\Omega_{0}\right) Y_{l}^{m}(\Omega)
$$

Accordingly, the coefficient $c_{l}^{m}\left(\Omega_{0}\right)$ is equal $Y_{l}^{m \star}\left(\Omega_{0}\right)$ and the final expression for the probability density function is:

$$
P\left(\Omega, \Omega_{0}, \tau\right)=\sum_{l, m} Y_{l}^{m \star}\left(\Omega_{0}\right) Y_{l}^{m}(\Omega) \exp \left[-\frac{\tau}{\tau_{r}}\right]
$$

Substituting Eq:92 in Eq:86 gives the final expression for the correlation function:

$$
G^{(q)}(\tau)=\frac{1}{4 \pi} \iint F^{(q)}(\Omega) F^{(q)^{\star}}\left(\Omega_{0}\right) \sum_{l, m} Y_{l}^{m \star}\left(\Omega_{0}\right) Y_{l}^{m}(\Omega) \exp \left[-\frac{\tau}{\tau_{r}}\right] \mathrm{d} \Omega \mathrm{d} \Omega_{0}
$$


Explicit calculations of the correlation functions for the different values of $q$ leads to the following expressions:

$$
G^{(0)}(\tau)=\frac{4}{5} \exp \left[-\frac{\tau}{\tau_{r}}\right] \quad G^{(1)}(\tau)=\frac{2}{15} \exp \left[-\frac{\tau}{\tau_{r}}\right] \quad G^{(2)}(\tau)=\frac{8}{15} \exp \left[-\frac{\tau}{\tau_{r}}\right]
$$

Inserting these expressions into Eq:87 and integration over the correlation time gives the final spectral densities:

$$
\begin{aligned}
J_{(p)}^{(0)}\left(\omega_{(p)}\right) & =\frac{4}{5} \frac{\tau_{r}}{1+\tau_{r}^{2}\left(\omega_{(p)}\right)^{2}} \\
J_{(p)}^{(1)}\left(\omega_{(p)}\right) & =\frac{2}{15} \frac{\tau_{r}}{1+\tau_{r}^{2}\left(\omega_{(p)}\right)^{2}} \\
J_{(p)}^{(2)}\left(\omega_{(p)}\right) & =\frac{8}{15} \frac{\tau_{r}}{1+\tau_{r}^{2}\left(\omega_{(p)}\right)^{2}}
\end{aligned}
$$

Considering a spectral density which depends on the electron Larmor frequency, the denominator is much greater than for spectral densities which depend on the nuclear Larmor frequency. Therefore, $J\left(\omega_{S}\right) \ll J\left(\omega_{I}\right)$ which validates the assumption made above.

Substituting the spectral densities into the relaxation rates for two nuclear spins (Eq:76 \& Eq:78) results in their final form:

$$
\begin{gathered}
\frac{1}{T_{1}}=\frac{3}{10}\left(\frac{\mu_{0}}{4 \pi}\right)^{2} \frac{\gamma_{k}^{2} \gamma_{l}^{2} \hbar^{2}}{r_{k l}^{6}}\left(\frac{\tau_{r}}{1+\tau_{r}^{2} \omega_{I}^{2}}+\frac{4 \tau_{r}}{1+4 \tau_{r}^{2} \omega_{I}^{2}}\right) \\
\frac{1}{T_{2}}=\frac{3}{20}\left(\frac{\mu_{0}}{4 \pi}\right)^{2} \frac{\gamma_{k}^{2} \gamma_{l}^{2} \hbar^{2}}{r_{k l}^{6}}\left(3 \tau_{r}+\frac{5 \tau_{r}}{1+\tau_{r}^{2} \omega_{I}^{2}}+\frac{2 \tau_{r}}{1+4 \tau_{r}^{2} \omega_{I}^{2}}\right)
\end{gathered}
$$

Considering the relaxation between electron and nucleus, electron relaxation is a factor which decreases the probability distribution in the correlation function because it is a competitive process to the contribution of the electron to the nucleus relaxation. Each electron which flips during the relaxation time is removed from the process. With a simple exponential decay of the relaxation with $\exp \left[-\tau / \tau_{s}\right]$ and $\tau_{s}$ as the electron relaxation time, the probability is given by:

$$
P\left(\Omega, \Omega_{0}, \tau\right)=\sum_{l, m} Y_{l}^{m \star}\left(\Omega_{0}\right) Y_{l}^{m}(\Omega) \exp \left[-\frac{\tau}{\tau_{r}}\right] \exp \left[-\frac{\tau}{\tau_{s}}\right]=\sum_{l, m} Y_{l}^{m \star}\left(\Omega_{0}\right) Y_{l}^{m}(\Omega) \exp \left[-\frac{\tau}{\tau_{c}}\right]
$$

Where $\tau_{c}=\left(\tau_{r}^{-1}+\tau_{s}^{-1}\right)^{-1}$ which can be expanded by considering a chemical exchange rate $\tau_{e x}$ or other contributing exponential processes to $\tau_{c}=\left(\tau_{r}^{-1}+\tau_{s}^{-1}+\tau_{e x}^{-1}+\ldots\right)^{-1}$ [Gueron1975]. Combining Eq:79 \& Eq 80 with the spectral densities calculated before, the relaxation rates for nuclei 
corresponding to direct dipole-dipole nucleus-electron relaxation are:

$$
\begin{gathered}
\frac{1}{T_{1}}=\frac{2}{5}\left(\frac{\mu_{0}}{4 \pi}\right)^{2} \frac{\gamma_{I}^{2} g_{e}^{2} \mu_{B}^{2}}{r_{I S}^{6}} S(S+1) \frac{\tau_{c}}{1+\tau_{c}^{2} \omega_{I}^{2}} \\
\frac{1}{T_{2}}=\frac{1}{15}\left(\frac{\mu_{0}}{4 \pi}\right)^{2} \frac{\gamma_{I}^{2} g_{e}^{2} \mu_{B}^{2}}{r_{I S}^{6}} S(S+1)\left(4 \tau_{c}+\frac{3 \tau_{c}}{1+\tau_{c}^{2} \omega_{I}^{2}}\right)
\end{gathered}
$$

The relaxation rates for two coupled unlike nuclei are obtained by substituting the spectral densities into $\mathrm{Eq}: 82$ \& Eq:83

$$
\begin{gathered}
\sigma_{I S}=\frac{1}{10}\left(\frac{\mu_{0}}{4 \pi}\right)^{2} \frac{\gamma_{I}^{2} \gamma_{S}^{2} \hbar^{2}}{r_{I S}^{6}}\left(-\frac{\tau_{r}}{1+\tau_{r}^{2}\left(\omega_{I}-\omega_{S}\right)^{2}}+\frac{6 \tau_{r}}{1+\tau_{r}^{2}\left(\omega_{I}+\omega_{S}\right)^{2}}\right) \\
\rho_{I}=\frac{1}{10}\left(\frac{\mu_{0}}{4 \pi}\right)^{2} \frac{\gamma_{I}^{2} \gamma_{S}^{2} \hbar^{2}}{r_{I S}^{6}}\left(\frac{\tau_{r}}{1+\tau_{r}^{2}\left(\omega_{I}-\omega_{S}\right)^{2}}+\frac{3 \tau_{r}}{1+\tau_{r}^{2} \omega_{I}^{2}}+\frac{6 \tau_{r}}{1+\tau_{r}^{2}\left(\omega_{I}+\omega_{S}\right)^{2}}\right)
\end{gathered}
$$

\subsubsection{Curie Spin Relaxation}

The Curie spin was derived before (Eq:30):

$$
\left\langle\mu_{S_{z}}\right\rangle=\frac{\mu_{B}^{2} g_{e}^{2} B_{0}}{3 k T} S(S+1)
$$

To derive the relaxation rates for Curie spin relaxation, first the Hamiltonian for a nucleus with $\vec{\mu}_{I}$ and the Curie spin which has just a $z$-component $\left\langle\mu_{S_{z}}\right\rangle$ is calculated. For the Curie spin the Hamiltonian is given by:

$$
\begin{aligned}
\widehat{\mathscr{H}}^{\mathrm{dip}, \mathrm{CS}}(t)= & \mathrm{D}^{\mathrm{dip}, \mathrm{CS}}\left[3\left(I_{z} \cos [\theta]+\sin [\theta]\left(I_{x} \cos [\varphi]+I_{y} \sin [\varphi]\right)\right)\left(\left\langle\mu_{S_{z}}\right\rangle \cos [\theta]\right)\right. \\
& \left.-I_{z} \cdot\left\langle\mu_{S_{z}}\right\rangle\right] \\
= & \mathrm{D}^{\operatorname{dip}, \mathrm{CS}}\left[I_{z}\left\langle\mu_{S_{z}}\right\rangle\left(3 \cos ^{2}[\theta]-1\right)+\frac{3}{2} \cos [\theta] \sin [\varphi] \exp [-i \varphi]\left(I_{+}\left\langle\mu_{S_{z}}\right\rangle\right)\right. \\
& \left.+\frac{3}{2} \cos [\theta] \sin [\varphi] \exp [i \varphi]\left(I_{-}\left\langle\mu_{S_{z}}\right\rangle\right)\right]
\end{aligned}
$$

With a modified coupling constant $\mathrm{D}^{\mathrm{dip}, \mathrm{CS}}=-\frac{\mu_{0}}{4 \pi} \frac{\gamma_{i} \hbar}{r_{I S}^{3}}$ for Curie spin relaxation. Following the same method to derive the Hamiltonian used above, the Curie spin Hamiltonian $\widehat{\mathscr{H}}$ dip,CS $(t)$ is:

$$
A^{(0)}=I_{z}\left\langle\mu_{S_{z}}\right\rangle
$$




$$
\begin{aligned}
A^{(1)} & =\frac{3}{2} I_{+}\left\langle\mu_{S_{z}}\right\rangle \\
\widehat{\mathscr{H}}^{\mathrm{dip}, \mathrm{CS}}(t) & =\mathrm{D}^{\mathrm{dip}, \mathrm{CS}} \sum_{-q}^{q} F^{(q)}(t) A^{(q)}
\end{aligned}
$$

The position functions stay the same. $A^{(2)}$ is missing in this equation since the Curie spin has just a $z$-component. Transforming the Hamiltonian to the interaction representation and calculating the double commutator for the longitudinal relaxation leads to:

$$
\sum_{q, p}\left[A_{(p)}^{(-q)},\left[A_{(p)}^{(q)}, I_{z}\right]\right] J_{(p)}^{(q)}\left(\omega_{(p)}^{(q)}\right)=9 I_{z}\left\langle\mu_{S_{z}}\right\rangle^{2} J^{(1)}\left(\omega_{I}\right)
$$

The Curie spin relaxation equation for longitudinal relaxation is:

$$
\frac{\partial}{\partial t} I_{z}=-\frac{\left(\mathrm{D}^{\mathrm{dip}, \mathrm{CS}}\right)^{2}}{\hbar^{2}} 9 I_{z}\left\langle\mu_{S_{z}}\right\rangle^{2} J^{(1)}\left(\omega_{I}\right)
$$

For the transverse relaxation time the double commutator is:

$$
\sum_{q, p}\left[A_{(p)}^{(-q)},\left[A_{(p)}^{(q)}, I_{x}\right]\right] J_{(p)}^{(q)}\left(\omega_{(p)}^{(q)}\right)=I_{x}\left\langle\mu_{S_{z}}\right\rangle^{2}\left(J^{(0)}(0)+\frac{9}{2} J^{(1)}\left(\omega_{I}\right)\right)
$$

The relaxation equation for transverse relaxation due to Curie spin relaxation now reads:

$$
\frac{\partial}{\partial t} I_{x}=-\frac{\left(\mathrm{D}^{\mathrm{dip}, \mathrm{CS}}\right)^{2}}{\hbar^{2}} I_{x}\left\langle\mu_{S_{z}}\right\rangle^{2}\left(J^{(0)}(0)+\frac{9}{2} J^{(1)}\left(\omega_{I}\right)\right)
$$

For Curie spin relaxation, only the correlation times corresponding to molecular motion are important because the Curie spin is already an averaged value and the electron relaxation rate has no influence [Gueron1975]. Substituting the spectral densities and the expectation value of the magnetic moment for the Curie spin $\left\langle\mu_{S_{z}}\right\rangle$ derived before, the final relaxation rates for Curie spin relaxation are:

$$
\begin{gathered}
\frac{1}{T_{1}}=\frac{6}{5}\left(\frac{\mu_{0}}{4 \pi}\right)^{2} \frac{\gamma_{I}^{2} g_{e}^{4} \mu_{B}^{4} B_{0}^{2}}{(3 k T)^{2} r_{I S}^{6}}(S(S+1))^{2} \frac{\tau_{r}}{1+\tau_{r}^{2} \omega_{I}^{2}} \\
\frac{1}{T_{2}}=\frac{1}{5}\left(\frac{\mu_{0}}{4 \pi}\right)^{2} \frac{\gamma_{I}^{2} g_{e}^{4} \mu_{B}^{4} B_{0}^{2}}{(3 k T)^{2} r_{I S}^{6}}(S(S+1))^{2}\left[4 \tau_{r}+\frac{3 \tau_{r}}{1+\tau_{r}^{2} \omega_{I}^{2}}\right]
\end{gathered}
$$

Since the equations for the previously derived direct dipole-dipole interaction (Eq:96 \& Eq:97) are based on the complete magnetic moment of the electron, there is a contribution of the Curie spin, which can be extracted by substituting $g_{e}^{2} \mu_{B}^{2} \frac{1}{3} S(S+1)$ with $\left(g_{e}^{2} \mu_{B}^{2} \frac{1}{3} S(S+1)-\left\langle\mu_{S_{z}}\right\rangle^{2}\right)$ which results in the following equations:

$$
\frac{1}{T_{1}}=\frac{6}{5}\left(\frac{\mu_{0}}{4 \pi}\right)^{2} \frac{\gamma_{I}^{2}}{r_{I S}^{6}}\left(g_{e}^{2} \mu_{B}^{2} \frac{1}{3} S(S+1)-\left\langle\mu_{S_{z}}\right\rangle^{2}\right) \frac{\tau_{c}}{1+\tau_{c}^{2} \omega_{I}^{2}}
$$




$$
\frac{1}{T_{2}}=\frac{1}{5}\left(\frac{\mu_{0}}{4 \pi}\right)^{2} \frac{\gamma_{I}^{2}}{r_{I S}^{6}}\left(g_{e}^{2} \mu_{B}^{2} \frac{1}{3} S(S+1)-\left\langle\mu_{S_{z}}\right\rangle^{2}\right)\left(4 \tau_{c}+\frac{3 \tau_{c}}{1+\tau_{c}^{2} \omega_{I}^{2}}\right)
$$

Combining Eq:104, Eq:105, Eq:106 \& Eq:107 and sorting for the part related to the Curie spin, restores the equation for direct dipole-dipole relaxation which are now independent from the Curie spin (Eq:96 \& Eq:97), and gives the final equations for Curie spin relaxation. In these following equations, it can be seen that Curie spin relaxation only takes place if the electron relaxation rate is much faster than the rotational correlation time.

$$
\begin{gathered}
\frac{1}{T_{1}}=\frac{6}{5}\left(\frac{\mu_{0}}{4 \pi}\right)^{2} \frac{\gamma_{I}^{2} g_{e}^{4} \mu_{B}^{4} B_{0}^{2}}{(3 k T)^{2} r_{I S}^{6}}(S(S+1))^{2}\left[\frac{\tau_{r}}{1+\tau_{r}^{2} \omega_{I}^{2}}-\frac{\tau_{c}}{1+\tau_{c}^{2} \omega_{I}^{2}}\right] \\
\frac{1}{T_{2}}=\frac{1}{5}\left(\frac{\mu_{0}}{4 \pi}\right)^{2} \frac{\gamma_{I}^{2} g_{e}^{4} \mu_{B}^{4} B_{0}^{2}}{(3 k T)^{2} r_{I S}^{6}}(S(S+1))^{2}\left[4 \tau_{r}+\frac{3 \tau_{r}}{1+\tau_{r}^{2} \omega_{I}^{2}}-4 \tau_{c}-\frac{3 \tau_{c}}{1+\tau_{c}^{2} \omega_{I}^{2}}\right]
\end{gathered}
$$

Similar to pseudocontact shifts, the relaxation rates due to paramagnetic relaxation depends on the vector between the nucleus and the paramagnetic center.

\subsection{Concluding Remarks}

In the previous sections, a complete derivation of the three major paramagnetic effects, starting from first principles, is given. The final equations for pseudocontact shifts, residual dipolar couplings and paramagnetic relaxation enhancement are dependent on distances and angles. Therefore, these structural parameters can be determined by measuring the afore mentioned paramagnetic effects.

The next planned step is to publish this derivation, thereby providing the community with a useful compendium. 


\subsection{Photochemically Induced Dynamic Nuclear Polarization (Photo CIDNP)}

The occurance of enhanced emissive or absorptive NMR signals during a photochemical reaction is called photo CIDNP. The source of this phenomenon is illumination of the NMR sample containing a photoactive substance, usually a dye, with the appropriate wavelength. The dye is excited from the singlet ground state $\left(S_{0}\right)$ to the first excited singlet state $\left(S_{1}\right)$. Subsequently intersystem crossing to the triplet state $\left(T_{1}\right)$ state occurs when the intersystem crossing rate is of the order of the $S_{1}$ lifetime. Phosphorescence results in the original state $\left(S_{0}\right)$ (Fig:2.6).

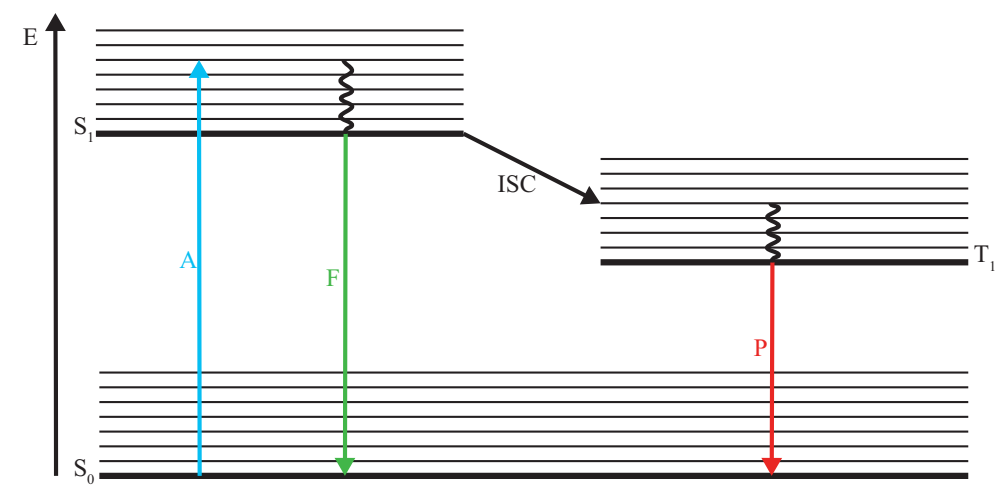

Figure 2.6: Electronic states and allowed transitions for a photoactive substance. $A=$ absorbance, $F=$ fluorescence, $P=$ phosphorescence, ISC $=$ intersystem crossing.

A competing process to phosphorescence takes place if there is another molecule (the quencher Q) in the sample which can quench the triplet state of the dye (D) via electron transfer. These two molecules then form a radical pair which is the source of the enhanced NMR signals.

$$
{ }^{3} \mathrm{D}+{ }^{1} \mathrm{Q} \rightarrow{ }^{3}\left[\mathrm{D}^{+}+\mathrm{Q}^{-}\right]
$$

\subsubsection{The Radical Pair Mechanism}

In general, there are two reaction pathways how a radical pair can be created, either thermically via cleavage of a chemical bond (mostly hydrogen abstraction) or photochemically via electron transfer. The spin multiplicity is conserved in both pathways and consequently, electron transfer creates a 
radical pair in the triplet state and the scission of a chemical bond in the singlet state. Therefore, in photo CIDNP experiments the radical pair is always created in the triplet state.

The crucial factor for the occurrence of photo CIDNP is intersystem crossing of the radical pair between the singlet state $|S\rangle$ and one of the three possible triplet states. For a two spin 1/2 system the allowed states are:

$$
\begin{array}{ll} 
& \left|\mathrm{T}_{+1}\right\rangle=|\alpha \alpha\rangle \\
\left|\mathrm{S}_{0}\right\rangle=\frac{1}{\sqrt{2}}(|\alpha \beta\rangle-|\beta \alpha\rangle) & \left|\mathrm{T}_{0}\right\rangle=\frac{1}{\sqrt{2}}(|\alpha \beta\rangle+|\beta \alpha\rangle) \\
& \left|\mathrm{T}_{-1}\right\rangle=|\beta \beta\rangle
\end{array}
$$

Diffusion drifts the two radicals apart and, in the special case of an NMR experiment with a strong external magnetic field, the three triplet states are not degenerate and, without spinflips, intersystem crossing can occur only between $\left|\mathrm{T}_{0}\right\rangle$ and $\left|\mathrm{S}_{0}\right\rangle$. The reason for this is that only these two states become degenerate by increasing the distance between the two electrons which is shown in Fig:2.7 [Goez1995].

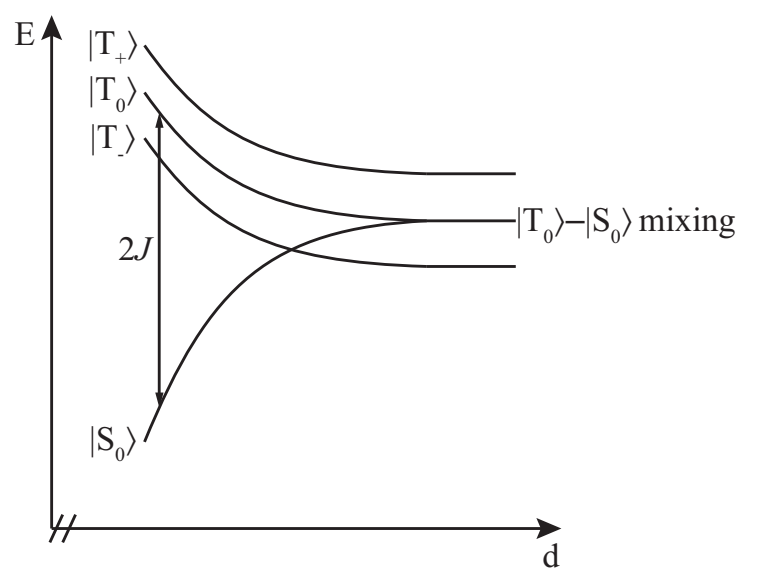

Figure 2.7: The distance dependency of the energies of the four electronic states.

The energy difference between $\left|\mathrm{S}_{0}\right\rangle$ and $\left|\mathrm{T}_{0}\right\rangle$ originates from the Coulomb interaction of indistinguishable electrons and is two times the exchange interaction $(J)$ [Gerthsen2006]:

$$
J=\frac{e^{2}}{4 \pi \varepsilon_{0}} \iint \frac{\Psi_{a}\left(\overrightarrow{r_{1}}\right) \Psi_{b}^{\star}\left(\overrightarrow{r_{2}}\right) \Psi_{b}\left(\overrightarrow{r_{2}}\right) \Psi_{a}^{\star}\left(\overrightarrow{r_{1}}\right)}{\left|\overrightarrow{r_{1}}-\overrightarrow{r_{2}}\right|^{2}} \mathrm{~d}^{3} r_{1} \mathrm{~d}^{3} r_{2}
$$


The exchange interaction is strongly distance dependent. $\Psi$ are the wave functions of the electrons, $e$ is the elementary charge and $\varepsilon_{0}$ is the vacuum permittivity.

Fig:2.8 illustrates a vector model of the following explanation in the complex plane. The two unpaired electrons of the radicals have different Larmor frequencies and for simplicity, the illustration takes place in a frame which rotates at the mean of the two Larmor frequencies. An electron transfer reaction is supposed to take place and therefore, due to the exchange interaction, the two radicals are a spin correlated radical pair which is in the triplet state (1). Due to diffusion, the two radicals ( $i$ and j) drift apart and the correlation between the molecules ends (2). Each electron rotates now with its Larmor frequency $\left(\omega_{i}\right.$ and $\left.\omega_{j}\right)(3)$. After a time interval, called the radical pair life time, the radicals either reencounter or become free radicals.

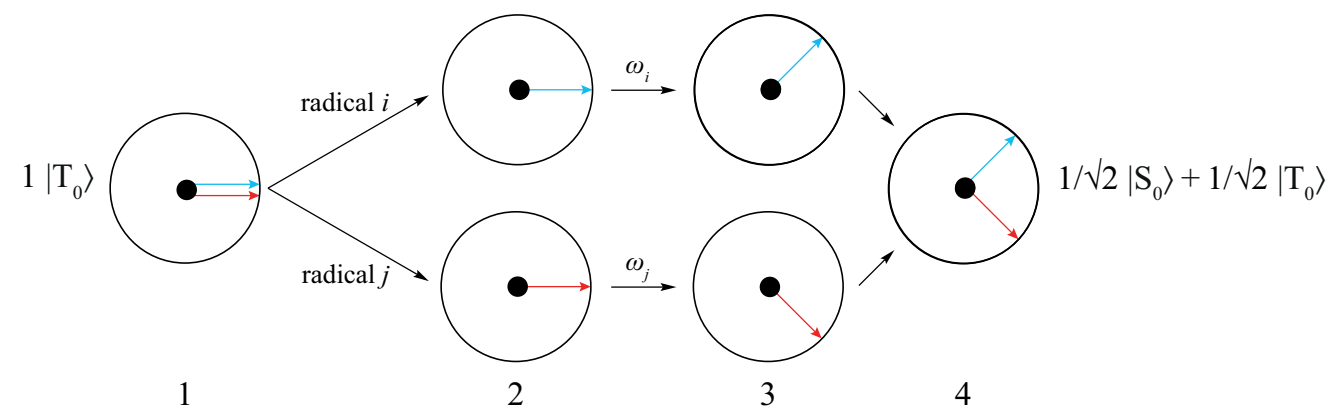

Figure 2.8: 1: Radical pair in the triplet state. 2: Seperation. 3: Larmor precession. 4: Spatial approach.

During the spatial approach (4) the system can be described by a superposition state of the singlet and triplet state:

$$
c_{S}\left|\mathrm{~S}_{0}\right\rangle+c_{t}\left|\mathrm{~T}_{0}\right\rangle
$$

The exchange interaction forces the two radicals upon reencounter to be either in a singlet or triplet state and the probability for one of these states is given by the square of the corresponding coefficient $\left(c_{s}, c_{t}\right)$. Starting with a triplet radical pair, these coefficients are [Closs1970]:

$$
\begin{aligned}
& c_{s}(t)=\sin \left[\frac{\Delta \omega}{2} t\right]=\sin \left[\frac{\Delta g \mu_{B} B_{0}}{2 \hbar} t\right] \\
& c_{t}(t)=\cos \left[\frac{\Delta \omega}{2} t\right]=\cos \left[\frac{\Delta g \mu_{B} B_{0}}{2 \hbar} t\right]
\end{aligned}
$$

$\Delta \omega$ is the difference of the Larmor frequencies which is equal to the intersystem crossing frequency and depends on the different $g$-values of the two radicals $(\Delta g)$. 
In the case where the molecules are orginally in the singlet state, recombination of the two can only occur if the forming radical pair is in the singlet state as well. Otherwise, seperation will take place again and another reencounter can occur or the two radicals will react via other pathways. The recombination product of the radical pair is called cage product. All other mechanisms yield escape products.

The probability to find the radical pair in one of the states depends on the lifetime of the radical pair and the intersystem crossing rate. The lifetime of a radical pair containing two small organic radicals is around $10^{-10}-10^{-11} \mathrm{~s}$, whereas the intersystem crossing rate is approximately $10^{8} \frac{\mathrm{rad}}{\mathrm{s}}$ [Adrian1977a which is not fast enough for the radicals to undergo intersystem crossing during the radical pair lifetime. Therefore, for diffusion controlled reactions, there must be a chance for the radicals to reencounter. Kaptein, and independently Adrian [Kaptein1969] [Adrian1970], applied Noyes' theory of the probability for a subsequent encounter of two molecules in the case of a diffusion controlled reaction [Noyes1954] to photo CIDNP to describe the average time interval until the first reencounter.

\subsubsection{Spin Sorting In A Radical Pair}

The probability of a radical pair to have a first reencounter was calculated with a random flight model by Noyes. With an encounter at time zero, this probability is given for each subsequent diffusion step $N$ by [Noyes1954]:

$$
f(N)=\frac{0.24}{(N+0.44)^{\frac{3}{2}}}
$$

This equation was obtained by fitting the calculated data and the values have no physical meaning. The probability of a successful recombination of two molecules $P$ for a triplet precursor is then given by [Adrian1970]:

$$
P=0.24 \frac{1}{3} k_{s} \int_{0}^{\infty} \frac{\sin \left[\frac{\Delta g \mu_{B} B_{0}}{2 \hbar} \tau N\right]^{2}}{(N+0.44)^{\frac{3}{2}}} \mathrm{~d} N
$$

$k_{s}$ is the probability for a recombination of two radicals encountering in a singlet state and $\tau$ is the translational correlation time. As explained above, in this model, intersystem crossing is only possible for $\left|\mathrm{T}_{0}\right\rangle$ and therefore a factor $1 / 3$ is introduced. Considering a spin $1 / 2$ nucleus coupled to the electron of the radical, the hyperfine splitting causes the Larmor frequency of the electron to differ for the two 
spin states of the nucleus. This effect, called spin-sorting, results in two different probabilities $P^{ \pm}$ dependent on the hyperfine splitting constant $A$. In Fig.2.9 this effect is shown for the case where the $\alpha$-state leads to a higher singlet character of the radical pair upon reencounter.

$$
P^{ \pm}=0.24 \frac{1}{3} k_{s} \int_{0}^{\infty} \frac{\sin \left[\left(\frac{\Delta g \mu_{B} B_{0}}{2 \hbar} \pm \frac{1}{4} A\right) \tau N\right]^{2}}{(N+0.44)^{\frac{3}{2}}} \mathrm{~d} N
$$

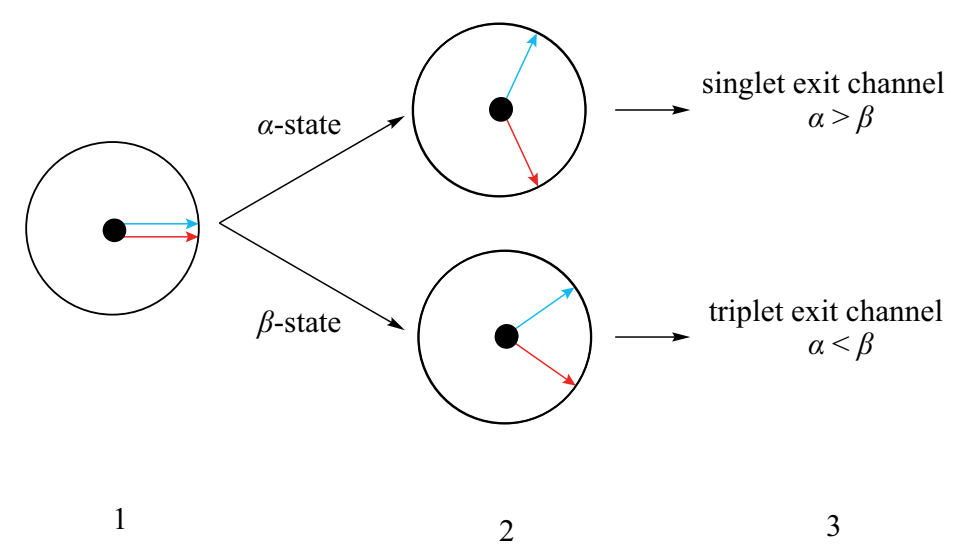

Figure 2.9: The effect of the hyperfine interaction on the intersystem crossing rate. In this example the hyperfine coupling increases the Larmor frequency of the $\alpha$-state.

Assuming a hyperfine coupling constant of 10 Gauss which corresponds to $1.76 \cdot 10^{8} \frac{\mathrm{rad}}{\mathrm{s}}$, a $400 \mathrm{MHz}$ spectrometer $(9.4 \mathrm{~T}), \Delta g=0.001$ and $\tau=10^{-10} \mathrm{~s}$ the different probabilities leads to:

$$
\Delta P^{ \pm}=P^{+}-P^{-}=8.5 \cdot 10^{-3} k_{s}
$$

This value represents nuclear hyperpolarization on the recombination products as long as $k_{s}$ is not very small $(<0.01)$. The equilibrium population difference at $298 \mathrm{~K}$ on a $400 \mathrm{MHz}$ spectrometer $(9.4 \mathrm{~T})$ is given by the Boltzmann distribution and around $6.4 \cdot 10^{-5}$. In this example the $\alpha$-state is overpopulated in the singlet state exit channel and the $\beta$-state is favoured in the triplet state exit channel, thus the escape products. Relaxation in triplet state molecules is much faster than in singlet state molecules and subsequently the equilibrium spin state is restored faster which allows the measurement of the hyperpolarized singlet state exit channel products. Furthermore, the radicals following the triplet exit channel normally yield different products than the recombination products. 
After these first numerical theories, more sophisticated approaches were developed. The probabilty of intersystem crossing between $\left|\mathrm{T}_{ \pm 1}\right\rangle$ and $\left|\mathrm{S}_{0}\right\rangle$, all possible encounters during the lifetime of the radical pair using the stochastic Liouville equation and the distance dependent exchange interaction are considered in the present theories [Kaptein1972] [Pedersen1973a] [Pedersen1973b] [Pedersen1974] [Pedersen1975] [Monchick1978] [Hore1979b] [Vollenweider1985] [Vollenweider1988].

\subsubsection{Kaptein's Rules}

Prediction of the observable NMR spectrum is possible with the following rules, first described by Kaptein and thus named Kaptein's rules [Kaptein1971]. Distinction between enhanced absorptive and emissive signals is possible with the net rule. A parameter $\Gamma^{\text {net }}$ is defined for which only the signs of the different parameters are taken into account:

$$
\begin{gathered}
\qquad \Gamma^{\text {net }}=\mu \varepsilon \operatorname{sign}(\Delta g) \operatorname{sign}(A) \\
\mu=\left\{\begin{array}{l}
+ \text { triplet precursor } \\
\text { - singlet precursor }
\end{array} \quad \varepsilon=\left\{\begin{array}{l}
+ \text { recombination products } \\
\text { - escape products }
\end{array}\right.\right.
\end{gathered}
$$

$\mu$ is plus for a triplet precursor and minus for a singlet precursor. $\varepsilon$ is plus for predicting recombination products and minus for escape products. A positive sign of $\Gamma^{\text {net }}$ gives an absorptive signal and a negative sign an emissive signal. For example, the signal form of a proton in the radical with the higher $g$-value $(\operatorname{sign}(\Delta g)=+)$ in the recombination products $(\varepsilon=+)$ for a singlet precursor $(\mu=-)$ with a positive hyperfine coupling constant $(\operatorname{sign}(A)=+)$ would be: $\Gamma^{\text {net }}=-+++=-$. Therefore, an enhanced emissive signal is predicted.

Considering the case where a second nucleus $(j)$ is coupled to the first nucleus $(i)$ with a scalar coupling constant $(J)$ and coupled to the electron, an expansion to the above rule is made. The two lines of the doublets in the NMR spectra show different behaviour and Kaptein's rule for multiplet effects is given by:

$$
\begin{gathered}
\Gamma^{\mathrm{multi}}=\mu \varepsilon \operatorname{sign}(\Delta g) \operatorname{sign}\left(A_{i}\right) \operatorname{sign}\left(A_{j}\right) \operatorname{sign}(J) \sigma \\
\sigma=\left\{\begin{array}{l}
+ \text { nuclei } i \text { and } j \text { are in the same radical } \\
- \text { nuclei } i \text { and } j \text { are in different radicals }
\end{array}\right.
\end{gathered}
$$




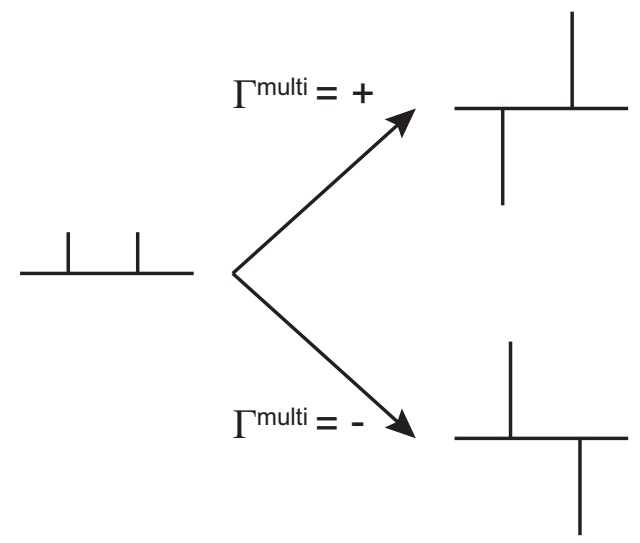

Figure 2.10: The predicted signal forms of Kaptein's multiplet effect.

A plus sign of $\Gamma^{\text {multi }}$ gives an E/A (emissive/absorptive) pattern of the dublets whereas a minus sign corresponds to an A/E pattern (Fig:2.10,

\subsubsection{Cross-Correlated Relaxation And Cross Relaxation}

After the creation of hyperpolarized species, cross-correlated relaxation between the magnetic moment of a nucleus and its chemical shift anisotropy (CSA) as well as cross relaxation between two magnetic moments of coupled nuclei lead to second order changes in the NMR spectra. These parameters can provide information about the structure and the dynamical behaviour of molecules. For the photo CIDNP systems used in this work, the assumption that only one spin (S) has a significant chemical shift anisotropy is made. Therefore, CSA-CSA cross-correlated relaxation can be neglected. The validity of this assumption will be reviewed in the results section (3.3.2). Considering the CSAdipole cross-correlated relaxation rate $\left(\delta_{S, I S}\right)(\mathrm{CSA}-\mathrm{DD})$, the following relaxation matrix is obtained [Goldman1984] [Kumar2000]:

$$
\frac{\partial}{\partial t}\left(\begin{array}{c}
I_{z} \\
S_{z} \\
2 I_{z} S_{z}
\end{array}\right)=-\left(\begin{array}{ccc}
\rho_{I} & \sigma_{I S} & 0 \\
\sigma_{I S} & \rho_{S} & \delta_{\mathrm{S}, I S} \\
0 & \delta_{\mathrm{S}, I S} & \rho_{\mathrm{ISIS}}
\end{array}\right)\left(\begin{array}{c}
\Delta I_{z} \\
\Delta S_{z} \\
2 I_{z} S_{z}
\end{array}\right)
$$

The dipole-dipole cross relaxation rate $\left(\sigma_{I S}\right)$ was derived before and the CSA-dipole cross correlated relaxation rate can be derived by a similar calculation with the previously stated chemical shift 
anisotropy tensor.

$$
\begin{gathered}
\sigma_{I S}=\frac{1}{10}\left(\frac{\mu_{0}}{4 \pi}\right)^{2} \frac{\gamma_{I}^{2} \gamma_{S}^{2} \hbar^{2}}{r_{I S}^{6}}\left[\frac{6 \tau_{c}}{1+\tau_{c}^{2}\left(\omega_{I}+\omega_{S}\right)^{2}}-\frac{\tau_{c}}{1+\tau_{c}^{2}\left(\omega_{S}-\omega_{I}\right)^{2}}\right] \\
\delta_{\mathrm{S}, I S}=\frac{2}{5}\left(\frac{\mu_{0}}{4 \pi}\right) \frac{\gamma_{I} \gamma_{S}^{2} \hbar B_{0}}{r_{I S}^{3}}\left[\frac{\tau_{c}}{1+\tau_{c}^{2} \omega_{S}^{2}} \Delta \sigma_{S}^{\mathrm{CSA}}\right]
\end{gathered}
$$

$\Delta \sigma_{S}^{\mathrm{CSA}}$ defines the geometrically weighted shielding anisotropy parameter with the principle components $\sigma_{x x}, \sigma_{y y}, \sigma_{z z}$ and the angles $\theta_{x x}, \theta_{y y}, \theta_{z z}$ between the axes of the CSA tensor and the internuclear vector:

$$
\Delta \sigma^{\mathrm{CSA}}=\frac{1}{2} \sigma_{x x}\left(3 \cos ^{2}\left[\theta_{x x}\right]-1\right)+\frac{1}{2} \sigma_{y y}\left(3 \cos ^{2}\left[\theta_{y y}\right]-1\right)+\frac{1}{2} \sigma_{z z}\left(3 \cos ^{2}\left[\theta_{z z}\right]-1\right)
$$




\section{Photo CIDNP}

\subsection{The Experimental Setup}

The source of light for all photo CIDNP experiments was a Laserworld BLUE-4500/445 laser operating with an output power of $4500 \mathrm{~mW}$ at $445 \mathrm{~nm}$. The diameter of the laser beam was reduced from $5.66 \mathrm{~mm}$ to $2.33 \mathrm{~mm}$ using a plano-convex (focal length: $100 \mathrm{~mm}$ ) and a bi-concave lense (focal length: $-15 \mathrm{~mm}$ ) with $50 \mathrm{~mm}$ distance between them. A Thorlabs PAF-X-15-PC-A collimator was used to couple the beam into a multimode fiber $(5 \mathrm{~m})$ with a core diameter of $600 \mu \mathrm{m}$. The coupling efficiency was measured with a photometer at the end of the fiber. After optimizing the positions of the lenses and the collimator, an output power of $3.4 \mathrm{~W}(76 \%)$ was measured. A mechanical shutter, controlled by the spectrometer software, was used to create laser pulses with a minimum duration of $5 \mathrm{~ms}$. Unless otherwise stated, all experiments were performed on a Bruker Avance Ultrashield Spectrometer (400 MHz) with Bruker Topspin 2.1 software. The setup is shown in Fig: 3.1 .

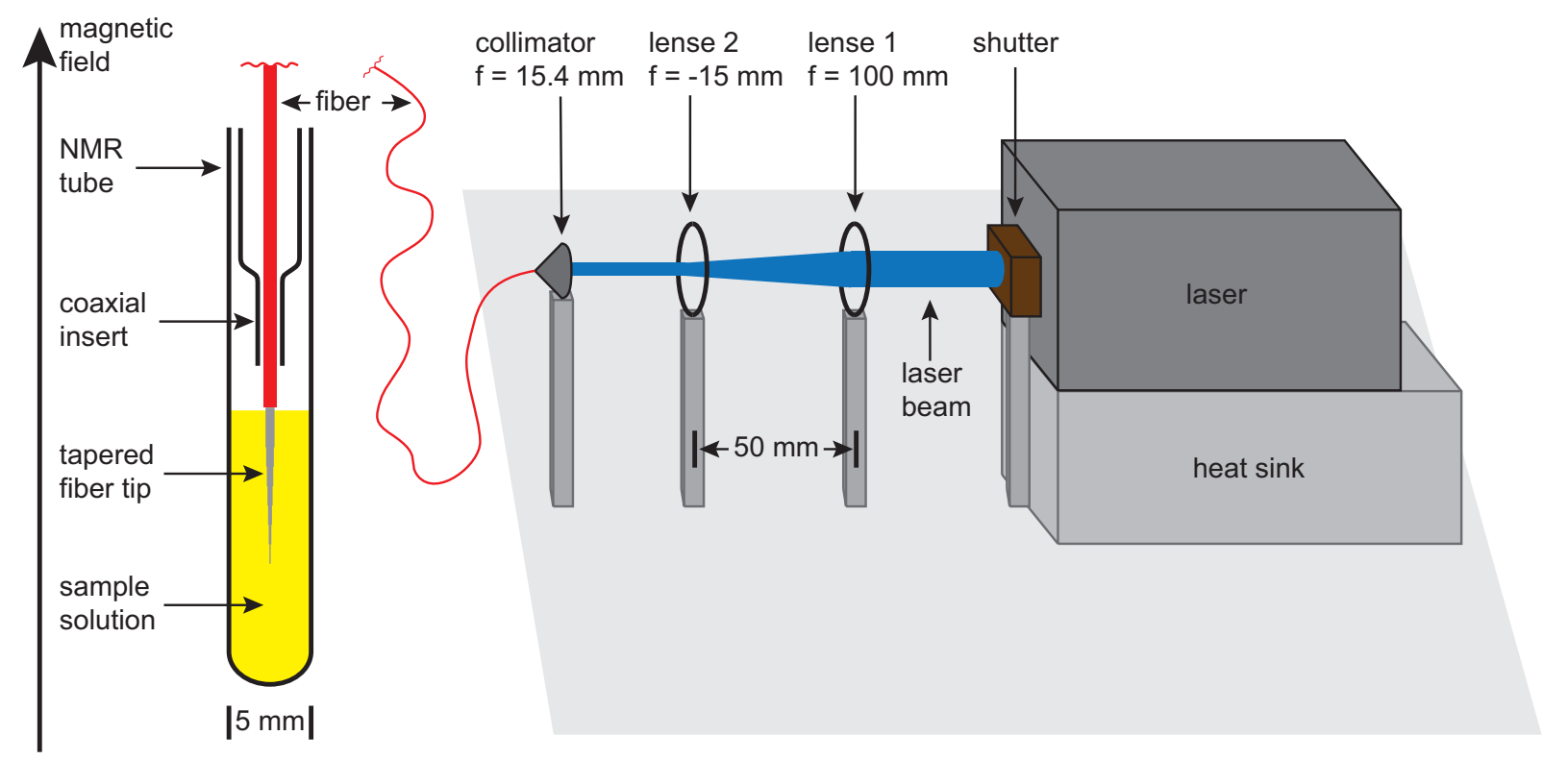

Figure 3.1: Schematic drawing of the photo CIDNP setup.

Homogeneous illumination of the sample was achieved with the tip of the fiber immersed in the sample solution (Fig:3.1). A Wilmad coaxial insert, with the tip removed, was used to hold the fiber 
in place. The end of the fiber was prepared following the procedure described by Kuprov \& Hore [Kuprov2004b]. $20 \mathrm{~mm}$ of the fiber's teflon coating were mechanically removed and the bare core was tapered by treatment with hydrofluoric acid (21\%), pyridine (9\%), sulfuric acid (20\%) and water $(50 \%)$ at $60^{\circ} \mathrm{C}$. During the procedure, the tip was stepwise extruded from the solution at a rate of $2.5 \mathrm{~mm}$ every $25 \mathrm{~min}$.

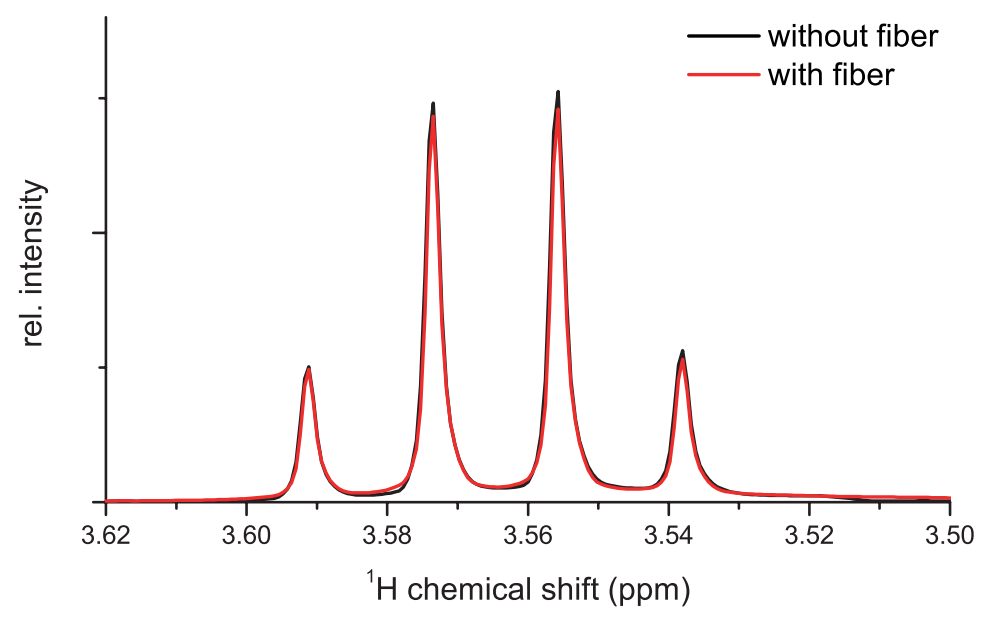

Figure 3.2: The signal of the $\mathrm{CH}_{2}$ group of ethanol with (red) and without (black) the coaxial insert and fiber.

All NMR experiments were performed using standard $5 \mathrm{~mm}$ NMR tubes, $600 \mu \mathrm{L}$ of the sample solution and $\mathrm{D}_{2} \mathrm{O}$ as solvent. The influence of the coaxial insert and the fiber on the quality of the spectra was tested on an ethanol sample in $\mathrm{D}_{2} \mathrm{O}(5 \%)$. An overlay of the $\mathrm{CH}_{2}$ signals with (red) and without (black) insert and fiber is shown in Fig:3.2. As made apparent by the overlaid spectra, the influence is negligible. The spectra were recorded in a simple laserpulse-mixing time- $90^{\circ} \mathrm{NMR}$ pulse experiment, as displayed in Fig3.3. Spectra with (light spectrum) and without (dark spectrum) laser pulse were directly compared. A squared cosine window function was applied to the obtained free induction decays before the Fourier transform. Subsequent phase and baseline correction gave the final spectra. Integration was performed by a Lorentzian line fitting to the peaks.

Due to the direct illumination of the sample, a heating of the sample solution was possible. The maximum temperature raise $\Delta T$ of $600 \mu \mathrm{L} \mathrm{D}_{2} \mathrm{O}\left(\rho=1.105 \mathrm{~g} / \mathrm{cm}^{3}\right.$ at $298.15 \mathrm{~K}$ [CRC2002] $)$ with a molar heat capacity at $298.15 \mathrm{~K}$ of $c_{m}=83.473 \frac{\mathrm{J}}{\mathrm{mol} \cdot \mathrm{K}}$ [Smirnova2006], a laser power of $P=3.4 \mathrm{~W}$ 


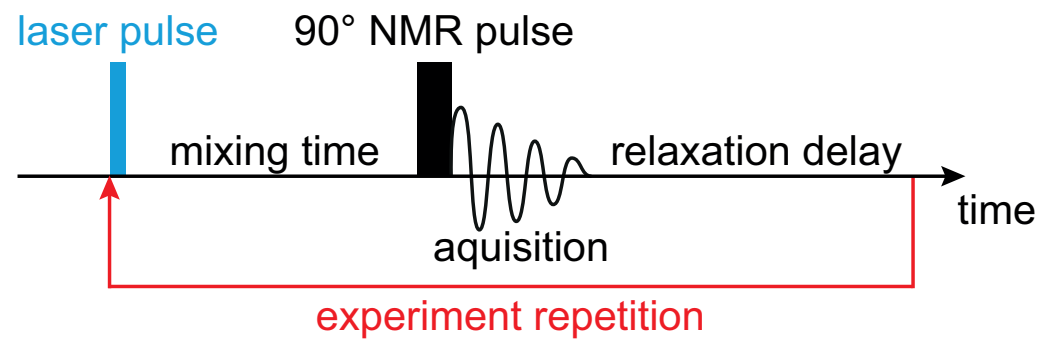

Figure 3.3: Pulse sequence used in all photo CIDNP experiments. An arbitrarily long laser pulse was followed by the mixing time and data aquisition was done directly after a $90^{\circ} \mathrm{NMR}$ pulse.

and a laser pulse duration of $20 \mathrm{~ms}$ is:

$$
\Delta T=\frac{P \cdot t}{\frac{\rho \cdot V}{M} c_{m}}=\frac{3.4 \frac{\mathrm{J}}{\mathrm{s}} 20 \cdot 10^{-3} \mathrm{~s} 20.03 \frac{\mathrm{g}}{\mathrm{mol}}}{1.105 \frac{\mathrm{g}}{\mathrm{cm}^{3}} 600 \cdot 10^{-3} \mathrm{~cm}^{3} 83.473 \frac{\mathrm{J}}{\mathrm{mol} \mathrm{K}}}=24.61 \cdot 10^{-3} \mathrm{~K}
$$

According to Eq:123, the overall sample heating is negligible. However, the heating is not induced uniformally and therefore the non-uniform sample heating can have an influence on the quality of the spectra. This aspect is further elaborated in the results section.

\subsection{The Investigated Molecules}

Flavin mononucleotide (FMN) was used as a dye in all of the experiments. For optimization of the setup and the first experiments, triethylenediamine (TEDA) was used as quencher (Fig:3.4). TEDA is a very small molecule with a diameter of less than $5 \AA$ (measured with Avogadro 1.0.3) and therefore has a short correlation time. Previously enhancement factors up to 7 were reported for time-resolved photo CIDNP experiments with 5-10 ns laser pulses [Kuprov2005]. Due to its properties, hyperpolarized TEDA was intended to be used as a subsequent polarizer molecule for biomacromolecules.

In this work, 3-fluoro-L-tyrosine (F-tyr) was mainly used as a quencher, whereas a few experiments were performed with L-tyrosine (Tyr) for comparison (Fig:3.4). All of the used systems follow the electron transfer pathway [Tsentalovich2002]. The $S_{1}$ - and $T_{1}$-lifetimes of FMN are approximately $5 \mathrm{~ns}$ and $1 \mathrm{~ms}$, respectively [Heelis1982] [Heelis1991], and the high triplet quantum yield of around 0.6 [vBerg2001] allows for the quenching of the dye in a diffusion controlled reaction. 
<smiles>Cc1cc2nc3c(=O)[nH]c(=O)nc-3n(C[C@H](O)[C@H](O)[C@H](O)COP(=O)([O-])O)c2cc1C</smiles>

25<smiles>N[C@@H](Cc1ccc(O)c(F)c1)C(=O)O</smiles>

26

F-tyr<smiles>N[C@@H](Cc1ccc(O)cc1)C(=O)O</smiles>

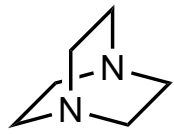

27

Tyr
28

TEDA

Figure 3.4: 25: Flavin mononucleotide. 26: 3-Fluoro-L-tyrosine. 27: L-Tyrosine. 28: Triethylenediamine.

Kuprov has shown that the relaxation of $\mathrm{H} 2$ and F3 of F-tyr can be described by a two spin system (Fig 3.4) and that only the fluorine exhibits a significant chemical shift anisotropy [Kuprov2004a]. The two spin system is therefore described by Eq:119. The validation of this model is given in the results section.

Recalling Eq:120, Eq:121, $\Delta \sigma_{\mathrm{F}}^{\mathrm{CSA}}$ in ppm, $B_{0}=9.3 \mathrm{~T}(400 \mathrm{MHz}$ spectrometer) and in the extreme narrowing limit $\left(\omega_{\mathrm{I}} \tau_{c} \ll 1\right)$, the dipole-dipole cross relaxation and CSA-dipole cross correlated relaxation rates are given by:

$$
\begin{gathered}
\sigma_{\mathrm{HF}}=\frac{1}{10}\left(\frac{\mu_{0}}{4 \pi}\right)^{2} \frac{\gamma_{\mathrm{H}}^{2} \gamma_{\mathrm{F}}^{2} \hbar^{2}}{r_{\mathrm{HF}}^{6}} 5 \tau_{c}=2.52 \times 10^{-49} \frac{\tau_{c}}{r_{\mathrm{HF}}^{6}} \frac{\mathrm{m}^{6}}{\mathrm{~s}^{2}} \\
\delta_{\mathrm{F}, \mathrm{HF}}=\frac{2}{5}\left(\frac{\mu_{0}}{4 \pi}\right) \frac{\gamma_{\mathrm{H}} \gamma_{\mathrm{F}}^{2} \hbar B_{0}}{r_{\mathrm{HF}}^{3}} \tau_{c} \Delta \sigma_{\mathrm{F}}^{\mathrm{CSA}}=6.65 \times 10^{-22} \frac{\tau_{c}}{r_{\mathrm{HF}}^{3}} \frac{\mathrm{m}^{3}}{\mathrm{~s}^{2}} \Delta \sigma_{\mathrm{F}}^{\mathrm{CSA}}
\end{gathered}
$$

The distance between F3 and H2 is $2.614 \AA$ (measured with Avogadro 1.0.3).

The photocycle for the reaction of F-tyr with FMN is shown in Fig. 3.5 FMN is excited from the singlet to the triplet state and subsequently quenched by F-tyr via electron transfer from the quencher to the dye. The created radical pair then separates into two doublet radicals, which either become free radicals, resulting in escape products, or reencounter. If a singlet state radical pair is formed upon 


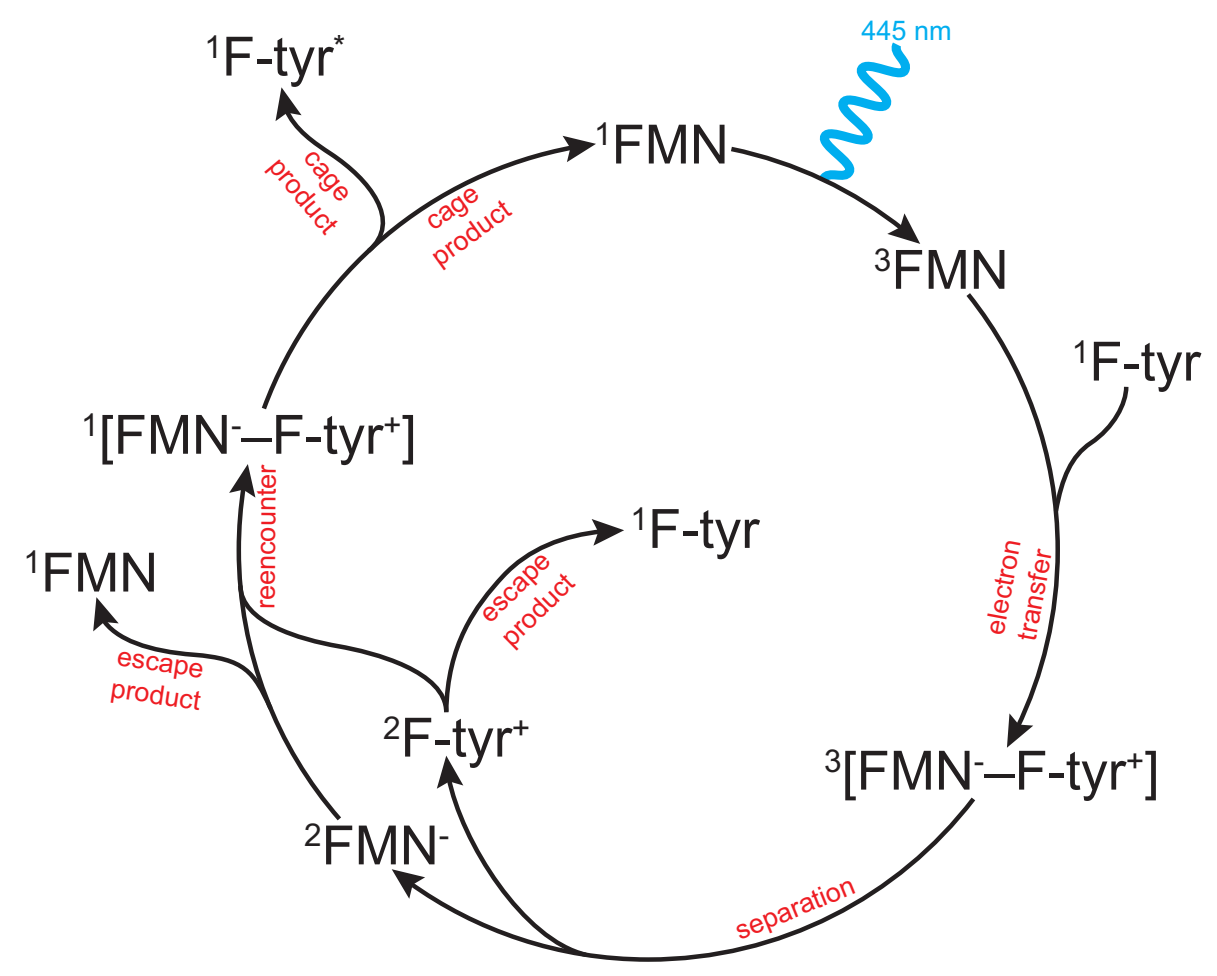

Figure 3.5: The photocycle for the reaction of FMN with F-tyr.

reencounter, the radicals react, forming singlet state hyperpolarized F-tyr ${ }^{\star}$ and singlet state FMN. A radical pair which reencounters in the triplet state will separate again.

Free radicals can also react to form other products which removes these molecules from the photocycle. This reaction pathway is called photobleaching and is one major problem of photoreactions which rely on a reaction pathway to recreate the reagents at the end of the reaction. Molecules which are removed from the photocycle due to photobleaching reduce the observed signal intensity and give raise to new signals in the NMR spectra. The amount of molecules which are removed per photocycle determines the lifetime of the sample. A long lifetime of the samples is necessary to record multidimensional NMR spectra, for which the runtime of the experiments last from several hours to days. 


\subsection{Results \& Analysis}

\subsubsection{Triethylenediamine}

Due to its symmetry, the 12 protons of triethylenediamine only show a single peak in ${ }^{1} \mathrm{H}-\mathrm{NMR}$ spectra (Fig:3.6). Determination of the enhancement factors of the photo CIDNP experiments was achieved by measuring dark and light spectra separately, followed by signal integration and subsequent division of the respective values. Unless otherwise stated, all experiments were performed at $298 \mathrm{~K}$, in $\mathrm{D}_{2} \mathrm{O}$ and at $\mathrm{pH} 7$.

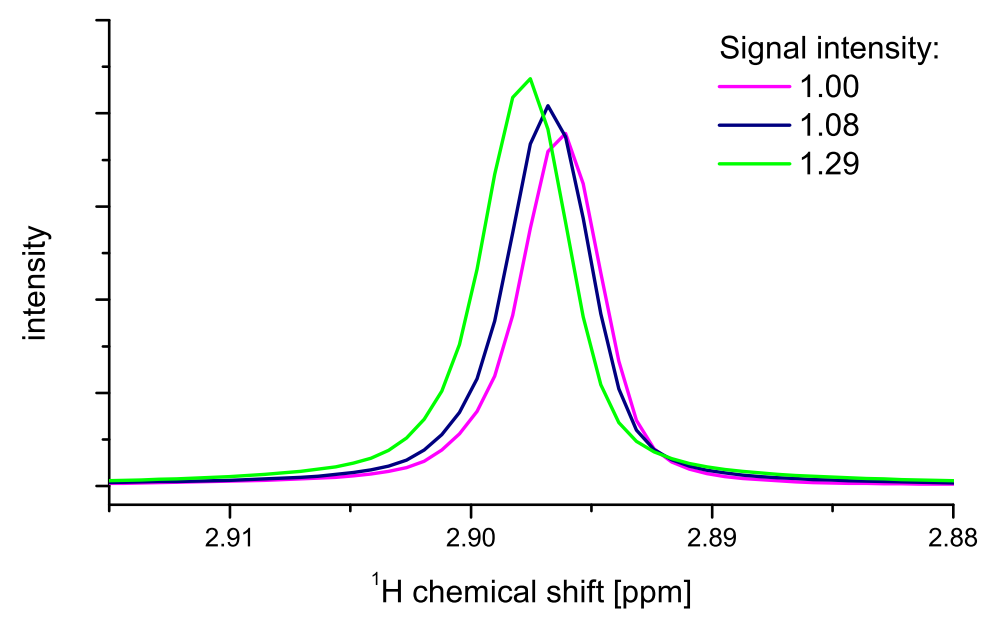

Figure 3.6: Example of the TEDA signal. Magenta: Dark spectrum. Dark Blue: Light spectrum (10 ms laser pulse). Green: Light spectrum (20 ms laser pulse).

Investigation of TEDA as a quencher for photo CIDNP started with optimization of the dye and quencher concentrations. The highest enhancements were observed at a concentration ratio TEDA/ FMN of 4 to $1 \mathrm{mmol} / \mathrm{L}$. Downscaling this ratio, lowering the concentration of FMN or increasing the concentration of TEDA decreased the observed enhancement. A higher FMN concentration increased the optical density of the sample and non-uniform sample heating led to significant line broadening of the TEDA signal.

The longitudinal relaxation time of hyperpolarized TEDA was determined using a $10 \mathrm{~ms}$ laser pulse excitation and subsequent observation of the time dependent signal decay (Fig:3.7). After the 
initial buildup, relaxation occurs at an exponential rate with a time constant of $2.02 \pm 0.18 \mathrm{~s}$. The corresponding non-polarized longitudinal relaxation time was determined by a standard inversion recovery experiment to $2.17 \pm 0.03 \mathrm{~s}$ (Fig:3.8). The similarity of these two rates is analyzed in the discussion section (3.4).

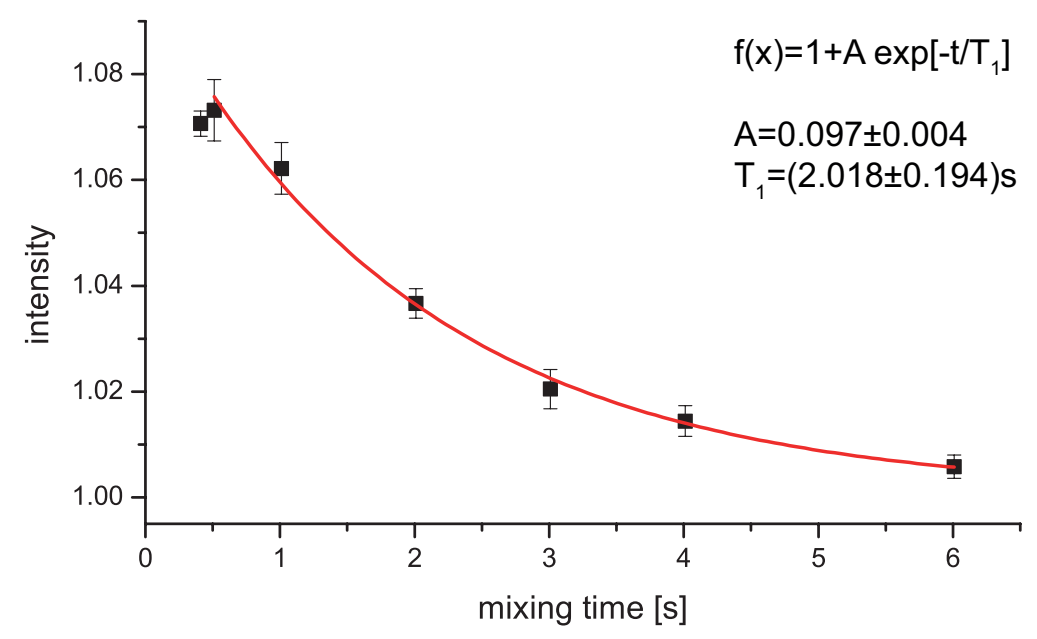

Figure 3.7: Determination of the longitudinal relaxation time after a $10 \mathrm{~ms}$ laser pulse.

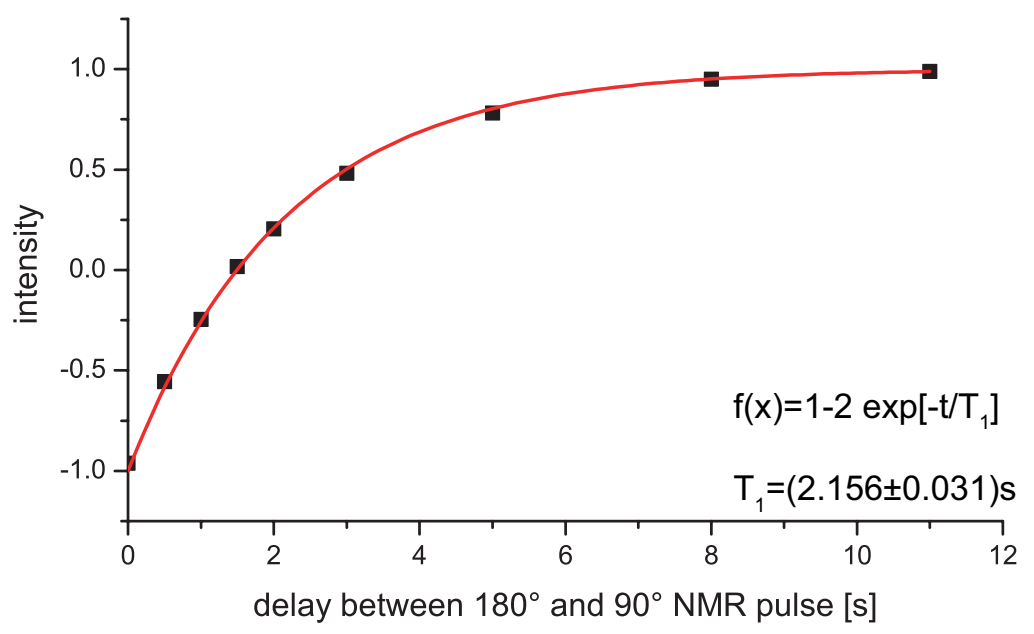

Figure 3.8: Determination of the longitudinal relaxation time with a standard inversion recovery experiment.

The average maximum enhancement of the TEDA signal after a $10 \mathrm{~ms}$ laser pulse was $7 \pm 2 \%$. This enhancement was observable after a mixing time of $0.5 \mathrm{~s}$ (Fig.3.7). Increasing the laser duration to 
$20 \mathrm{~ms}$ gave an enhancement of $30 \pm 1 \%$. This was, however, reflected mostly in a broadening of the TEDA signal and not by an increase of the signal to noise ratio (Fig 3.6 . Further increase of the pulse duration worsened this effect, resulting in a decrease of the signal to noise ratio compared to shorter laser pulses.

The change in the chemical shift of the TEDA signals in Fig. 3.6 results from non-uniform sample heating caused by the laser pulse. The heating broadens and shifts the water signal, which is the reference signal for the chemical shift values.

Enhancement factors for TEDA after a $10 \mathrm{~ms}$ laser pulse were also measured for two more temperatures. Raising the temperature to $318 \mathrm{~K}$ slightly decreased the observed enhancement to $6 \pm 1 \%$, whereas the enhancement factor for $278 \mathrm{~K}$ was significantly increased to $11 \pm 1 \%$ (Fig:3.9).

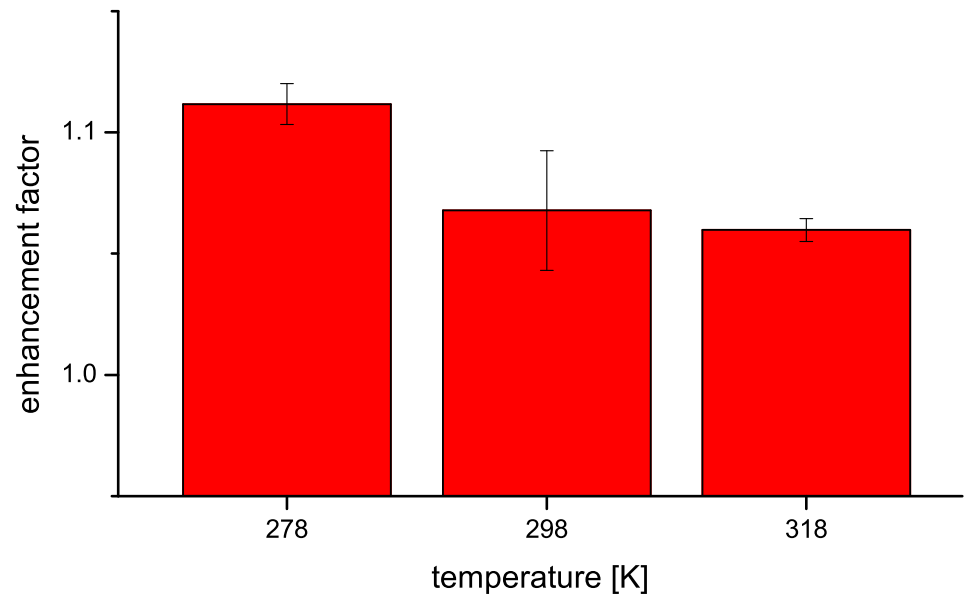

Figure 3.9: Average enhancement of the TEDA signal for three different temperatures.

Over the course of several experiments on the same sample, a reduction in enhancement factors was observed, which is attributed to the effect of photobleaching (see section 3.3.4). 


\subsubsection{3-Fluorotyrosine}

The previously derived relaxation matrix for F-tyr reads as follows (Eq:119):

$$
\frac{\partial}{\partial t}\left(\begin{array}{c}
H_{z} \\
F_{z} \\
2 H_{z} F_{z}
\end{array}\right)=-\left(\begin{array}{ccc}
\rho_{\mathrm{H}} & \sigma_{\mathrm{HF}} & 0 \\
\sigma_{\mathrm{HF}} & \rho_{\mathrm{F}} & \delta_{\mathrm{F}, \mathrm{HF}} \\
0 & \delta_{\mathrm{F}, \mathrm{HF}} & \rho_{\mathrm{HF}}
\end{array}\right)\left(\begin{array}{c}
\Delta H_{z} \\
\Delta F_{z} \\
2 H_{z} F_{z}
\end{array}\right)
$$

The cross relaxation and cross-correlated relaxation rates in the extrem narrowing limit were given before as $(\mathrm{Eq}: 124$ \& $\mathrm{Eq}: 125)$ :

$$
\begin{aligned}
\sigma_{\mathrm{HF}} & =2.52 \times 10^{-49} \frac{\tau_{c}}{r_{\mathrm{HF}}^{6}} \frac{\mathrm{m}^{6}}{\mathrm{~s}^{2}} \\
\delta_{\mathrm{F}, \mathrm{HF}} & =6.65 \times 10^{-22} \frac{\tau_{c}}{r_{\mathrm{HF}}^{3}} \frac{\mathrm{m}^{3}}{\mathrm{~s}^{2}} \Delta \sigma_{\mathrm{F}}^{\mathrm{CSA}}
\end{aligned}
$$

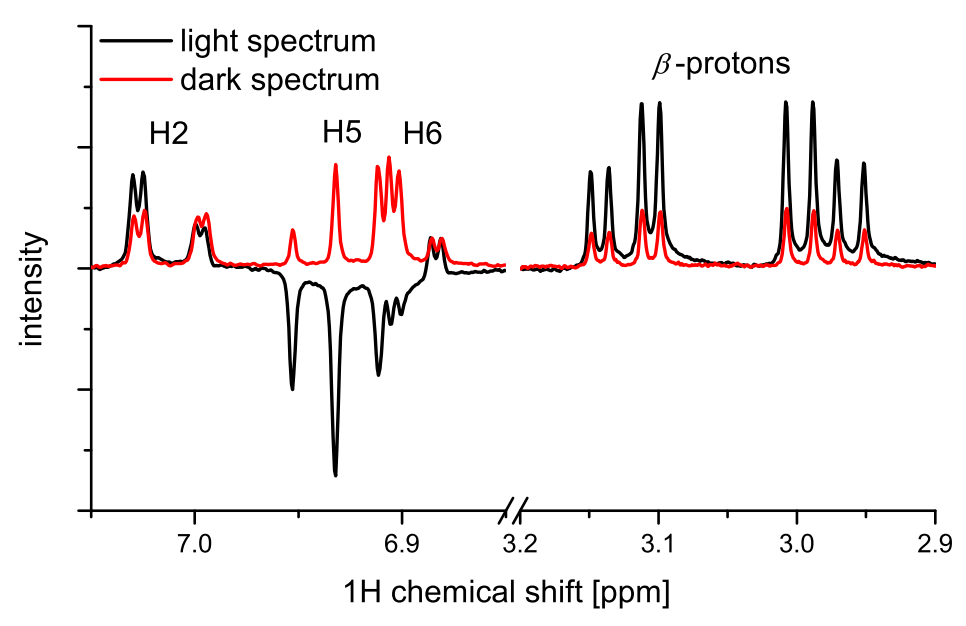

Figure 3.10: Comparison of the F-tyr dark and light spectrum after a $100 \mathrm{~ms}$ laser pulse, $100 \mathrm{~ms}$ mixing time and $\mathbf{~ 9 0}^{\circ} \mathrm{NMR}$ pulse. Shown are the aromatic and beta protons.

For photo CIDNP with F-tyr as a quencher, the best results were observed for a F-tyr/FMN concentration ratio of 4 to $0.2 \mathrm{mmol} / \mathrm{L}$. The lower FMN concentration and therefore the lower optical density allowed for the application of longer laser pulses than for TEDA without inducing extensive line broadening. An overlay of a light and a dark spectrum of F-tyr is shown in Fig: 3.10 . 
The general behaviour of F-tyr during photo CIDNP experiments was investigated by performing sets of experiments and varying one parameter per set. The main parameters for the experiments are the laser pulse duration and the mixing time. The ${ }^{19} \mathrm{~F}$-signal dependency on the laser pulse duration is shown in Fig: 3.11 with an initial buildup rate of $79 \mathrm{~s}^{-1}$ (red line). It was expected that the fluorine polarization reaches a steady state at which the photo CIDNP build-up rate is balanced with the ralaxation rates. The lower polarization achieved by a $6.4 \mathrm{~s}$ laser pulse compared to a $3.2 \mathrm{~s}$ pulse presumably originates from the previously mentioned photobleaching effect (see section 3.3.4).

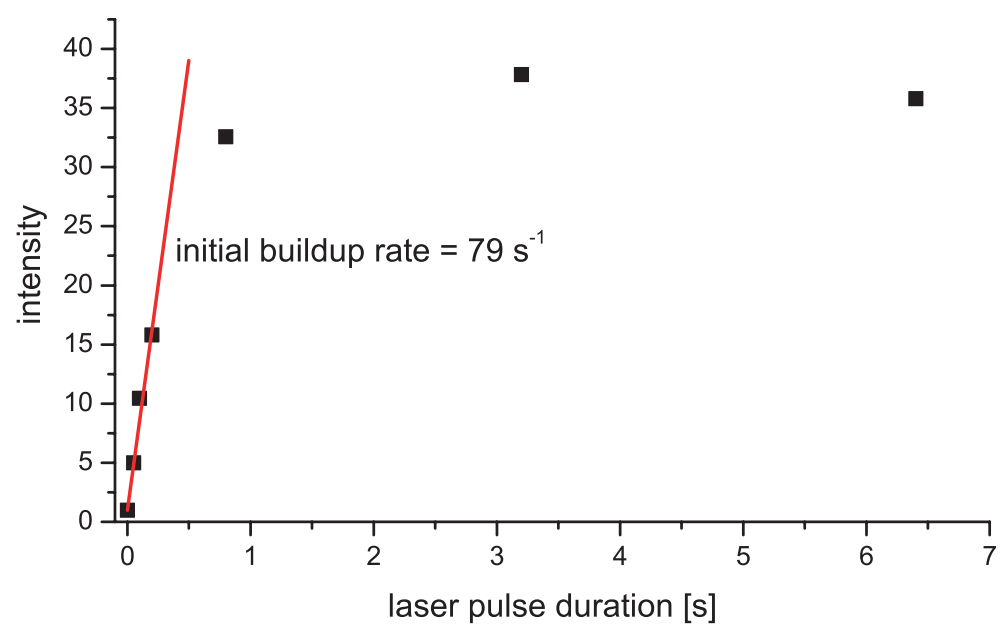

Figure 3.11: The fluorine signal intensity of F-tyr for different laser pulse duration. The initial buildup rate was determined to $78 \mathrm{~s}^{-1}$ (red line).

All following spectra were recorded using samples, that had previously been purged with argon for $20 \mathrm{~min}$, which slightly increased the enhancement factors and lifetimes of the samples. The dependency of the $\mathrm{H} 2$ proton of F-tyr on the mixing time after a $100 \mathrm{~ms}$ laser pulse is shown in Fig:3.12, The signal is a doublet of doublets with $J=12 \mathrm{~Hz}(\mathrm{~F} 3)$ and $J=2 \mathrm{~Hz}$ (H6). The different behaviour of the two parts of the flourine induced doublet indicates the buildup of longitudinal twospin order $2 H_{z}^{(2)} F_{z}$.

After these first experiments, the assumption that the relaxation behaviour of $\mathrm{H} 2$ and F3 is sufficently described by considering just these two spins was to be validated. To do this, ${ }^{1} \mathrm{H}-$ and ${ }^{19} \mathrm{~F}$ spectra with different mixing times were recorded (Fig:3.3).

The relative signal intensities of the light spectrum to the corresponding dark spectrum, dependent 


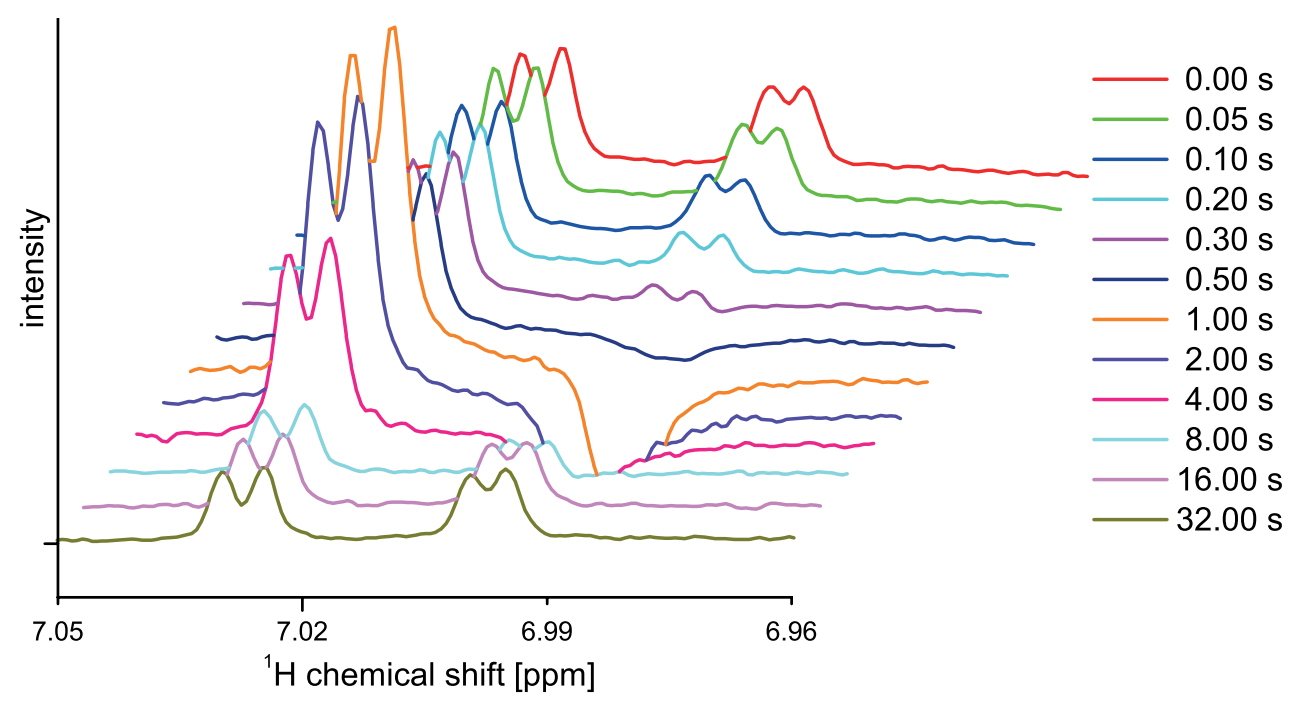

Figure 3.12: The observed aromatic $\mathrm{H} 2$ signal of $\mathrm{F}$-tyr, after a $100 \mathrm{~ms}$ laser pulse, dependent on the mixing time.

on the mixing time, are shown in Figs $3.13 \& 3.14 \& 3.15$ The fluorine signal is a doublet of doublets with $J=12 \mathrm{~Hz}(\mathrm{H} 2)$ and $J=8 \mathrm{~Hz}(\mathrm{H} 5)$. Therefore, the intensity of the $2 H_{z}^{(2)} F_{z}$ mode was calculated from the difference of the two doublets, separated by $12 \mathrm{~Hz}$.

Neglecting the initial build up rates, the system is described by three coupled differential equations with five variables: one cross relaxation, one cross-correlated relaxation and three auto relaxation rates $(\mathrm{Eq}: 126)$. Following the Monte Carlo method, 100 datasets of the three graphs were created with an assumed standard deviation of $5 \%$ for each value. The three equations were fitted to the three graphs (black lines) of each dataset, respectively, with an explicit Runge-Kutta method. The variables were adjusted to minimize the root-mean-square deviation (RMSD) between the fit and the graphs using the differential evolution method. The mean and RMSD of the resulting 100 sets of the five relaxation rates are shown in Tab1 All calculations were performed with Mathematica 8.0.4.0 and the included packages.

With the resulting cross relaxation and cross-correlated relaxation rates, the rotational correlation time $\tau_{c}$ and the shielding parameter $\Delta \sigma_{\mathrm{F}}^{\mathrm{CSA}}$ were easily calculated and given in Tab1, 
In the following figures the red squares represent the experimental data and the black lines the fitting curves.

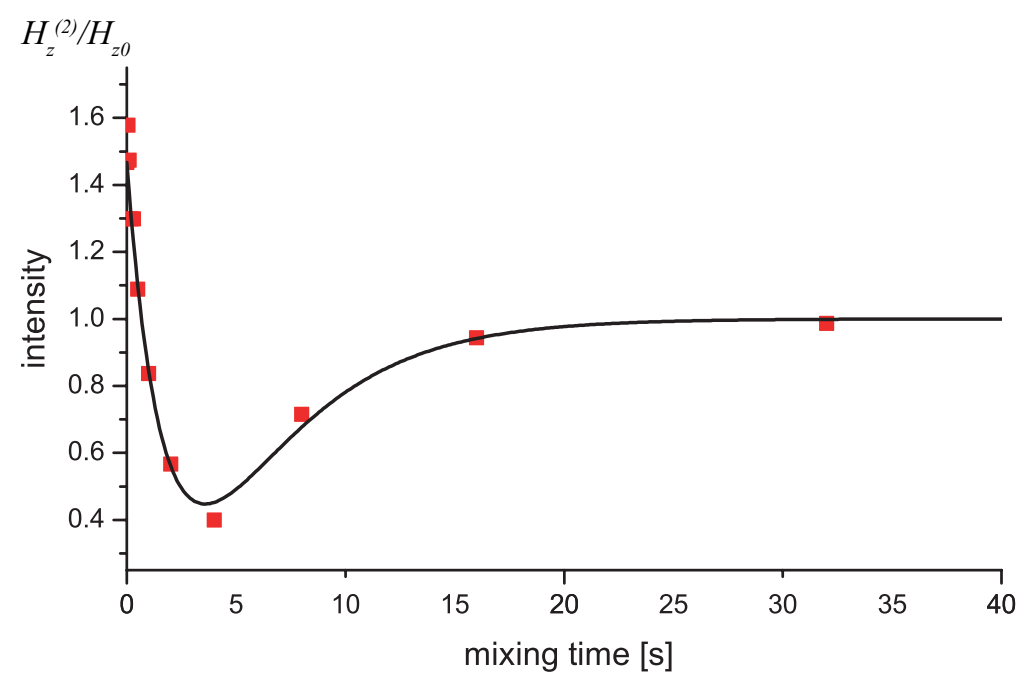

Figure 3.13: The observed relative $\mathbf{H} 2$ signal intensity of $\mathrm{F}$-tyr after a $100 \mathrm{~ms}$ laser pulse, dependent on the mixing time.

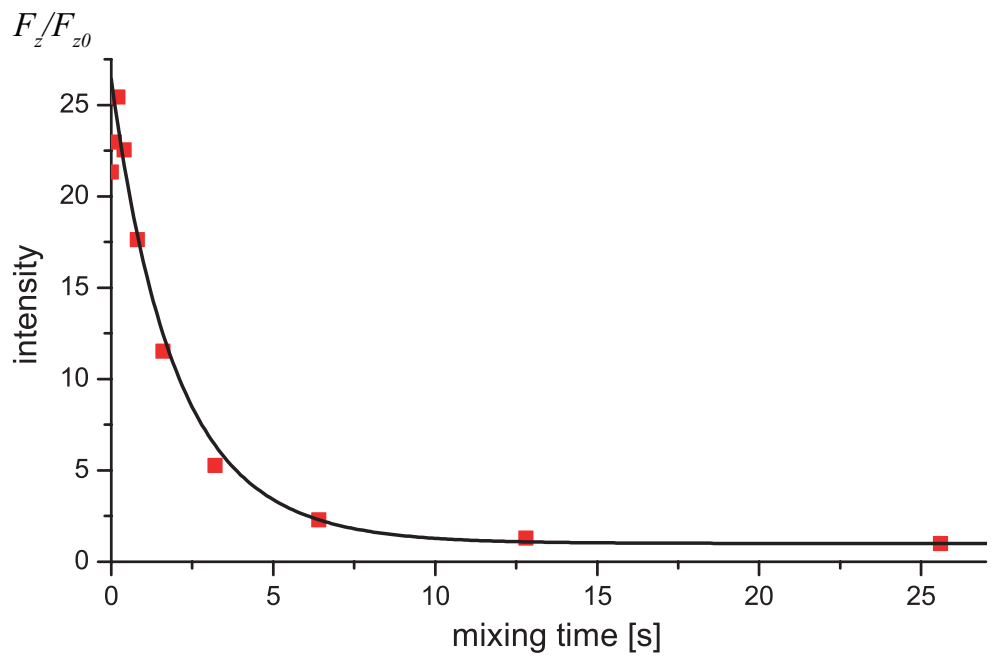

Figure 3.14: The observed relative $F 3$ signal intensity of F-tyr after a $100 \mathrm{~ms}$ laser pulse, dependent on the mixing time. 


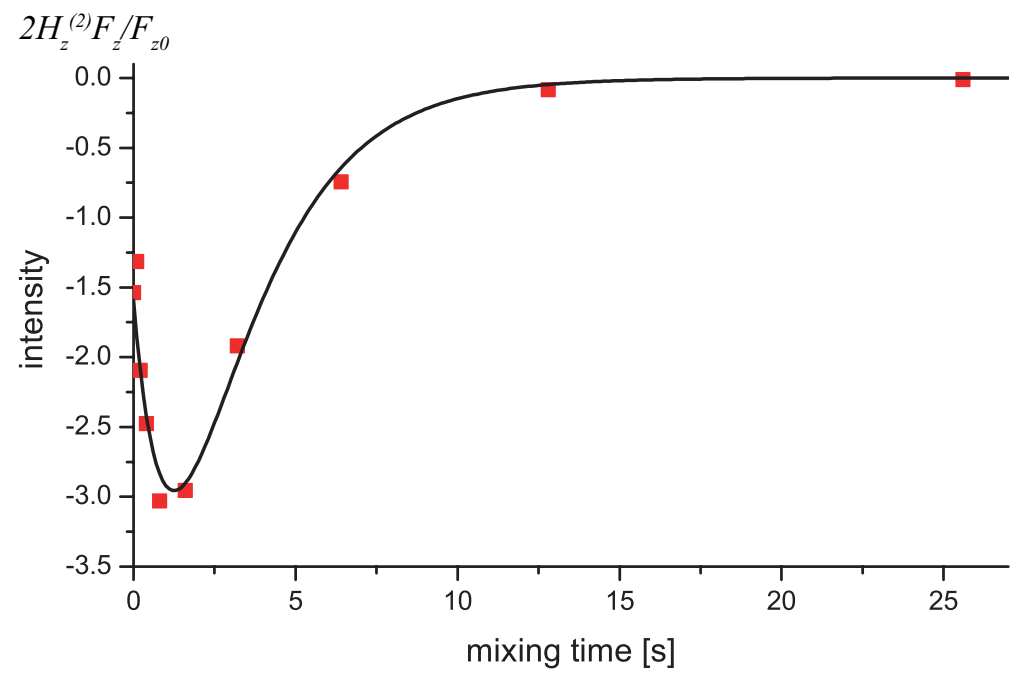

Figure 3.15: The calculated relative $2 H_{z}^{(2)} F_{z}$ signal intensity of $\mathrm{F}$-tyr after a $100 \mathrm{~ms}$ laser pulse, dependent on the mixing time.

\begin{tabular}{c|c}
$\rho_{\mathrm{H}}$ & $(328 \pm 32) \cdot 10^{-3} \mathrm{~s}^{-1}$ \\
\hline$\rho_{\mathrm{F}}$ & $(572 \pm 22) \cdot 10^{-3} \mathrm{~s}^{-1}$ \\
\hline$\rho_{\mathrm{HF}}$ & $(899 \pm 74) \cdot 10^{-3} \mathrm{~s}^{-1}$ \\
\hline$\sigma_{\mathrm{HF}}$ & $(40 \pm 1) \cdot 10^{-3} \mathrm{~s}^{-1}$ \\
\hline$\delta_{\mathrm{F}, \mathrm{HF}}$ & $(220 \pm 15) \cdot 10^{-3} \mathrm{~s}^{-1}$ \\
\hline$\tau_{c}$ & $50 \pm 2 \mathrm{ps}$ \\
\hline$\Delta \sigma_{\mathrm{F}}^{\mathrm{CSA}}$ & $118 \pm 8 \mathrm{ppm}$
\end{tabular}

Table 1: The resulting parameters for photo CIDNP of F-tyr. The significance of these values is discussed in the next chapter (3.4). 


\subsubsection{Tyrosine}

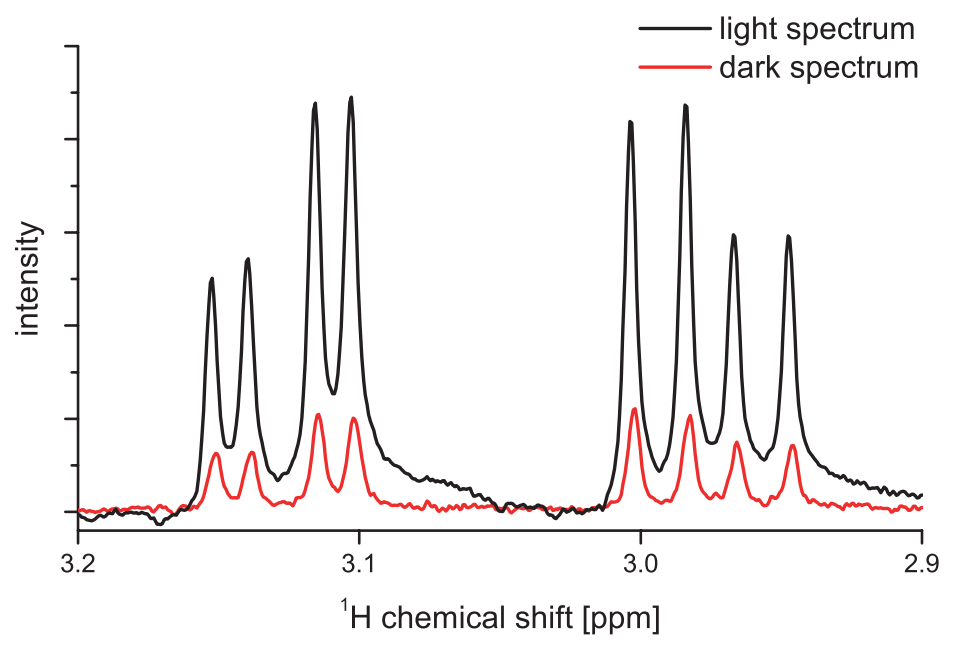

Figure 3.16: The $\beta$-protons of tyrosine were enhanced by a factor of 4.6 for the first experiment.

Experiments with tyrosine resulted in signal enhancement for $\mathrm{H} 2 / \mathrm{H} 6, \mathrm{H} 3 / \mathrm{H} 5$ and the $\beta$-protons (Fig:3.16). In Fig:3.17, the enhancement factors in three consecutive single laser pulse experiments of a freshly prepared sample are shown. The first enhancements are, as can be seen in the figure, significantly higher than for F-tyr, yet, with each laser pulse the enhancement factors are drastically decreased. The negative enhancement factors for the H3/H5 protons are a result of a negative hyperfine coupling constant.

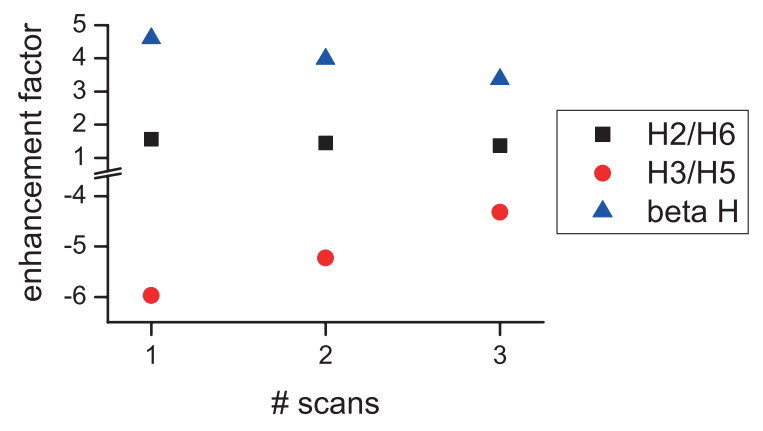

Figure 3.17: Enhancement factors of the three proton groups of tyrosine for three consecutive experiments. 


\subsubsection{Photobleaching}

The direct influence of photobleaching on the signal intensity can be seen in Fig 3.18 . The aromatic region of F-tyr is shown for 10 selected spectra out of a series of 91 experiments, recorded using the same sample. Every 10 scans, the enhancement factors are reduced by approximately $6 \%$, i.e. after 91 scans, the enhancement factor for the $\beta$-protons was reduced from 2.7 to 1.8 .

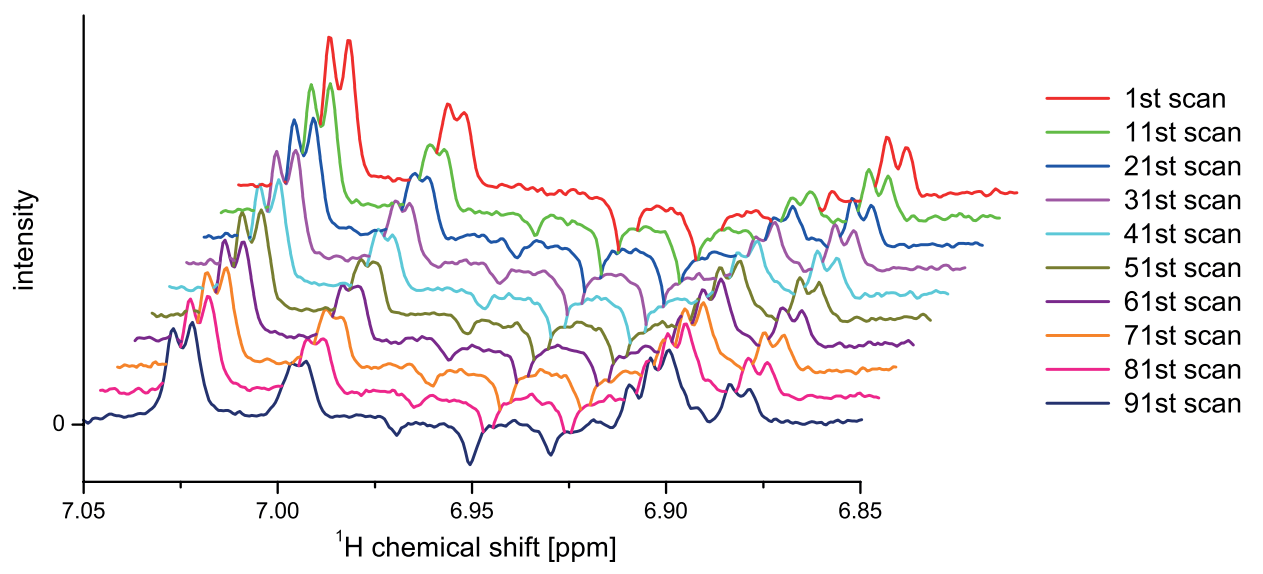

Figure 3.18: The aromatic region of $F$-tyr after every 10 scans with a $100 \mathrm{~ms}$ laser pulse, $50 \mathrm{~ms}$ mixing time and 5 min relaxation delay.

In a first attempt to increase the lifetime of the F-tyr sample, dissolved oxygen was removed by purging with argon. 20 min of argon flushing allowed for execution of 20 experiments using $100 \mathrm{~ms}$ laser pulses before a decrease in the enhancement factors was observed. Further removal of oxygen by longer purging with argon, application of reduced pressure and ultrasonic sound reversed the increase in the sample lifetime. This observation indicates that oxygen, despite its negative effects, serves as a repair molecule (see section 3.4). Addition of oxidizing and/or reducing agents (hydrogen peroxide and/or sodium ascorbate) had no influence on the sample lifetime. The lifetime of a TEDA sample showed the same behaviour as the F-tyr sample, whereas for a Tyr sample, no increase of the sample lifetime was observed.

As a consequence of the limited number of experiments executable before the photobleaching effects become too severe, the sample has to be replaced by a fresh one after an accumulated illumination of $2 \mathrm{~s}$. Furthermore, longer laser pulses immediately induce an error in the enhancement factors, 
which can be seen in Fig:3.11 for the fluorine polarization dependency on the laser pulse duration.

For none of the three investigated quenchers, a degradation product was observed in the NMR experiments. All mechanisms leading to a decrease in enhancement factors remove FMN from the photocycle.

\subsection{Discussion \& Outlook}

The NMR signal of triethylenediamine was reproducibly enhanced by approximately 7\% (10 ms laser pulse) and 30\% (20 ms laser pulse). The determined longitudinal relaxation rate for photo CIDNP experiments $(2.02 \pm 0.19 \mathrm{~s})$ is in excellent agreement with the non-hyperpolarized longitudinal relaxation rate $(2.16 \pm 0.03 \mathrm{~s})$. This shows that for TEDA no other relaxation mechanisms, such as cross relaxation or cross-correlated relaxation, has to be considered. The small enhancements also allow for a high repetition rate of the experiments.

TEDA has a high quenching rate constant with flavins of approximately $1.5 \cdot 10^{9} \mathrm{M}^{-1} \mathrm{~s}^{-1}$ which is higher than for Tyr $\left(9.8 \cdot 10^{8} \mathrm{M}^{-1} \mathrm{~s}^{-1}\right.$ [Porcal2003] [Heelis 1991]). Nevertheless, the reported high enhancement factors, achieved after nanosecond laser pulses [Kuprov2005], were not observable with the experimental setup used in this work. The presumed reason is degenerate electron transfer between the radicals and their diamagnetic form which leads to a nearly complete cancellation of measurable polarization differences between the two product pathways [Roth1974].

Therefore, a different mechanism to create the hyperpolarization has to be utilized in order to subsequently employ TEDA as a polarizer for other molecules. Time-resolved photo CIDNP, which was mentioned above, or solution state dynamic nuclear polarization may prove to be applicable. The latter technique uses stable radicals and microwave irridation to saturate the electron spin and subsequent cross relaxation then induces polarization transfer to the nuclei [Hausser1968] [Lingwood2011].

A limiting factor for the conduction of photo CIDNP experiments, for TEDA as well as for F-tyr and Tyr, is the lifetime of the employed samples. Photobleaching induces a decrease in the enhancement factors after several seconds of accumulated laser illumination. All of the previously described attempts to tackle this obstacle, led to just a minor increase in the lifetimes of the samples. 
The enhancement factors for the $\beta$-protons and H3/H5 of tyrosine are very high, compared to F-tyr, with approximately 5 and -6 , respectively. These enhancements are, however, only observable with a freshly prepared sample. The significant decrease in enhancement with every scan indicates the lack of singlet recombination products and renders tyrosine unsuitable for photo CIDNP. The negative value of the $\mathrm{H} 3 / \mathrm{H} 5$ enhancement factor reflects the negative hyperfine coupling constants between the protons and the electron. This negative hyperfine coupling constant was predicted by Kuprov \& Hore, based on density functional theory calculations [Kuprov2004a].

The most promising results were obtained with F-tyr. The assumption that the relaxation can be sufficiently described by a two spin system (H2 and F3) was tested. Fitting the three coupled differential equations to the graphs for the three polarization modes yielded satisfying values for all relaxation rates. As appearing from the graphs (Figs:3.13 \& $3.14 \& 3.15$, the relaxation is very well described by the fitting.

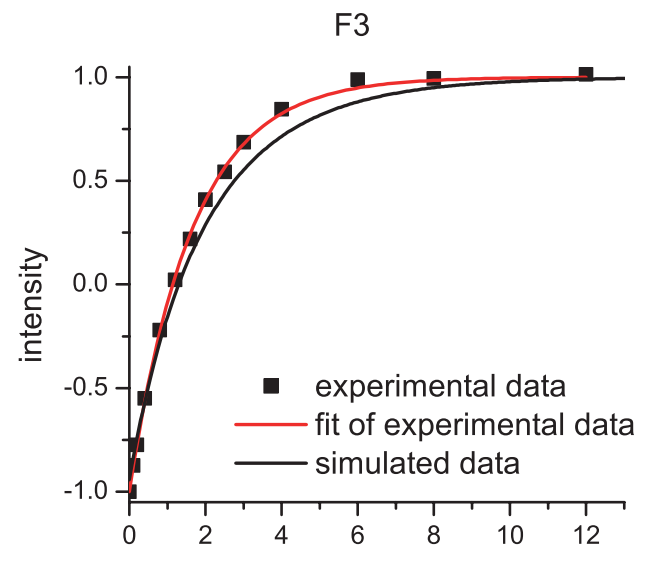

[s]

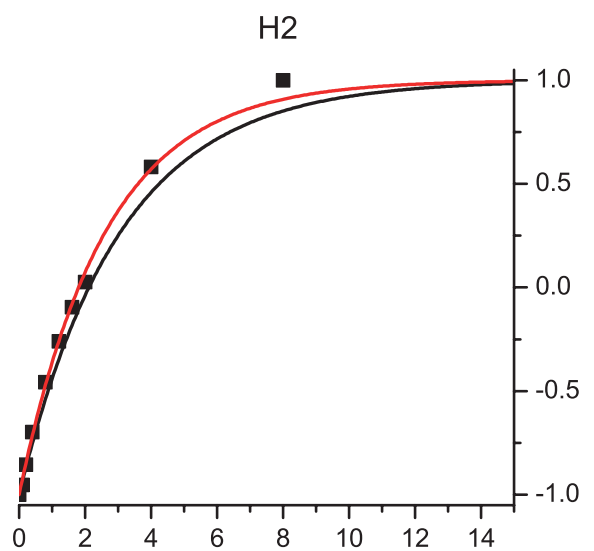

[s]

Figure 3.19: Comparison of the experimental data (black squares) for an inversion recovery experiment and the simluated data for the same starting magnetization (black lines) for F3 (left) and H2 (right).

Nevertheless, in the extreme narrowing limit, the auto relaxation rate is expected to be approximately twice the cross relaxation rate. The fact that the determined ratio in this work is approximately nine indicates that the two spins are involved in other relaxation mechanisms. However, since the fitting of the experimental data with the $3 \times 3$ matrix was possible, it is assumed that it is valid to 
incorporate all other relaxation mechanisms into the auto relaxation rates. To validate this assumption, longitudinal relaxation rates for $\mathrm{F} 3$ and $\mathrm{H} 2$ were measured with a standard inversion recovery experiment $\left(\mathrm{F} 3:(608 \pm 6) \cdot 10^{-3} \mathrm{~s}^{-1} ; \mathrm{H} 2:(385 \pm 10) \cdot 10^{-3} \mathrm{~s}^{-1}\right)$. The obtained data was compared to the simulated evolution of the magnetization described by the $3 \times 3$ matrix (3.19). The very well agreement of the curves validates the incorporation of other relaxation mechanisms into the auto relaxation rates.

With the obtained cross relaxation rate $\left(\sigma_{H F}=(40 \pm 1) \cdot 10^{-3} \mathrm{~s}^{-1}\right)$, the rotational correlation time of F-tyr is $50 \pm 2$ ps. This value is much smaller than the correlation time determined by Kuprov \& Hore, following the same method (106 $\pm 15 \mathrm{ps})$. Reported values for the correlation time of tyrosine in aqueous solution are around $40 \mathrm{ps}$, which is in close proximity to the value determined in this work [Lakowicz1983] [Nordlund1986] [Harms1997]. The determined shielding parameter $(118 \pm 8 \mathrm{ppm})$ is a little higher than reported values for multisubstituted fluorobenzenes $(81-88$ ppm) [Dorai2001], whereas Kuprov \& Hore reported $39 \pm 8$ ppm. Direct illumination using the tapered fiber tip gave a significantly higher fluorine buildup rate $\left(79 \mathrm{~s}^{-1}\right)$ and enhancement factor (25) compared to illumination from above the sample (34 s ${ }^{-1}$ and 14) when applying a $500 \mathrm{~ms}$ laser pulse [Kuprov2004a].

Photo CIDNP active amino acids are mainly tyrosine, tryptophane and histidine. Several investigations of protein surfaces with solvent exposed photo CIDNP active amino acids and dynamics in form of relative sidechain mobilities have been reported [Hore1993] [Ivanov2011]. Nevertheless, Ftyr mutated proteins were only investigated once by Kuprov et al. to explain unusual phase behaviour of fluorine in photo CIDNP experiments [Kuprov2007]. The high enhancement factors for F-tyr, achieved in this work, can be used to increase the efficiency and sensitivity of photo CIDNP experiments with proteins. Achieving a high level polarization of fluorine, followed by a cross relaxation mechanism, can increase the NMR signals of otherwise "photo CIDNP invisible" nuclei.

Photobleaching in form of degradation of the dye and/or the quencher is a major obstacle in the field of photo CIDNP. A straightforward solution to this problem would be to exchange the sample after a given number of experiments, yet this proves to be a bothersome and time-consuming procedure, as both, the fiber and the spectrometer, have to be adjusted after each exchange. Minor aberrations in the adjustments of the setup or just slight variations in the sample concentrations can significantly impair 
the comparison of spectra measured on different samples. As a consequence, a sample reinjection system, which exchanges the sample solution without removing the fiber and sample tube from the spectrometer, was developed by Kuprov [Kuprov2005a]. Despite its benefits, installation of such a system was not practicable in our laboratory and is not applicable in the case of limited amounts of the sample.

Time-resolved photo CIDNP setups with laser pulse durations of $5-15$ ns use either a sample reinjection system [Kuprov2005] [Goez2005] or suffer from comparable degradation rates (up to 20\% within 40 laser pulses) [Morozova2004] [Kiryutin2007].

Two more methods to avoid extensive photobleaching were developed in the Cavagnero group. Two dimensional NMR spectra were recorded with low laser powers $(500 \mathrm{~mW})$ to increase the signal to noise ratio of tryptophane residues [Sekhar2009]. With a laser power this low, the enhancement factors between 1.2 and 2.1 were significantly smaller compared to $6.5 \pm 0.3$ achieved with a laser power of $4 \mathrm{~W}$ [Lyon1999]. Nevertheless, the observed degradation of only 10\% after 600 laser pulses is extremely small.

The most recent and promising approach is the utilization of a tri-enzyme system. In regular samples, molecular oxygen is useful by reoxidizing hydrated FMN $\left(\mathrm{FMNH}_{2}\right)$, which is the main degradation product, yet also reacts with intermediate products of the photocycle, thereby reducing the enhancement factors. In this approach, glucose oxidase and catalase are employed to very efficiently remove oxygen from the sample solution, whereas nitrate reductase adopts the useful function of oxygen and reoxidizes $\mathrm{FMNH}_{2}$ [Lee2013]. The concentrations of the three enzymes are less than $1 \mu \mathrm{mol} / \mathrm{L}$ and therefore negligible compared to the concentration of the investigated molecule (usually between 1 and $4 \mathrm{mmol} / \mathrm{L}$ ). With a laser power of $750 \mathrm{~mW}$, the observed enhancement factors were 4.5-fold higher than without the tri-enzyme system. After 320 laser pulses, these enhancements were reduced by $20 \%$, yet still higher than without the three enzymes.

\subsubsection{Concluding Remarks}

The following short summary will conclude this topic.

A photo CIDNP setup was successfully planned, built and adjusted, including the laser completely with heatsink, mechanical shutter, lenses, collimator and modified fibers. This setup was tested by 
reproducing previously reported results for FMN and F-tyr. Even without a sample reinjection system, the obtained results for the rotational correlation time (50 $\pm 2 \mathrm{ps})$ are in much better agreement with literature values $(35-40 \mathrm{ps})$ than the photo CIDNP derived values by Kuprov (106 \pm 15 ps). The major problem which has to be solved, in order to improve the reliability, the sample lifetime and therefore the usefulness of this technique, is the degradation of the samples as a result of photobleaching. Addition of oxidizing or reducing agents showed no impact on the lifetimes of the samples, yet as the very efficient tri-enzyme system developed by the Cavagnero group clearly shows, reoxidization of hydrated FMN can lad to a significant increase in enhancement factors and sample lifetimes. The next steps for this topic are to adopt the tri-enzyme system and to test its applicability to F-tyr modified proteins and subsequent cross relaxation of hyperpolarized fluorines. 


\section{Tagging Strategy For DNA}

The lack of natural occuring sulfur moieties in DNA molecules leads to the need of synthetically introduced sulfur atoms when using tags based on disulfide bridges as connection. Modification of the type of linkage between the DNA and a tag poses an alternative to this approach. Either of this methods requires a modification of the DNA strand which is achievable at the phosphate backbone or at one or more of the nucleosides. In this work, synthetically modified nucleosides were used. The Cys-Ph-TAHA tag, recently developed in our group (Fig,4.1), was the initial molecule for all approaches [Peters2011].

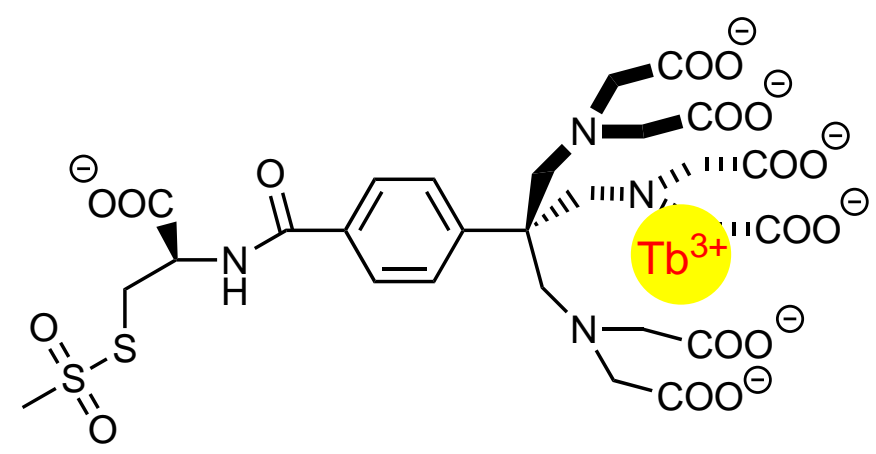

16

Figure 4.1: Cys-Ph-TAHA tag coordinating $\mathbf{T b}^{3+}$.

The chelating TAHA part of the tag was first described by Viguier and has high stability constants when binding lanthanide ions $(\log K=14.85)$ which is the main prerequisite for using this chelator in paramagnetic NMR [Viguier2001].

All DNA molecules were synthesized by IBA. Synthesis of small polynucleotides is nowadays performed in automated DNA synthesizers from the $3^{\prime}$-end to the $5^{\prime}$-end. The first nucleoside is bound to a solid silica phase, a method first described over 30 years ago [Matteucci1981]. Each nucleoside has a dimethoxytrityl protection group bound to the 5'-oxygen, which is easy to cleave. The concentration of the cleaved protection group and the coupling efficiency are routinely determined by UV-spectroscopy. Coupling to the next nucleoside occurs at the $3^{\prime}$-position via a (2-cyanoethyl)diisopropylphosphoramidite moiety, which is easily activated [Caruthers1991]. A complete synthesis 
cycle is devided into the following phases [Reese2005]:

- Detritylation:

Addition of trichloroacetic acid (3\% in dichloromethane) cleaves the dimethoxytrityl group.

- Coupling:

Addition of tetrazole and the next nucleoside (in acetonitrile) protonates the diisopropylamine group, which is subsequently substituted with the tetrazole. The activated phoshphorus species reacts with the $5^{\prime}$-oxygen of the previous nucleotide to give a phosphite-triester.

- Capping:

The excess molecules, which did not react, are removed from the reaction cycle by esterification with acetic anhydride.

- Oxidation:

The phosphite-triester is oxidized to the corresponding phosphate-triester using iodine.

After the synthesis, all remaining protection groups on the nucleosides as well as the cyanoethyl groups bound to the phosphorus are released by treatment with ammonia. This step also cleaves the DNA from the solid phase.

The sequence of the used polynucleotide is given in Fig 4.2 .

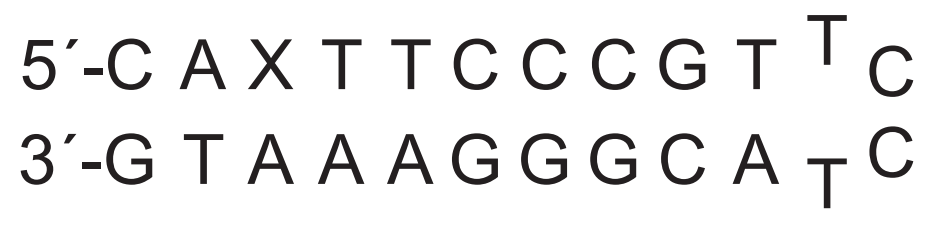

Figure 4.2: The sequence of the test DNA strand. The $X$ marks the position of the modification. In the wildtype DNA, a thymidine occupies this position.

Utilization of a self-complementary polynucleotide rather than two single strands avoids stoichiometric problems. Unpaired single strands would lead to a second set of signals in the NMR spectra. The position of the modification (Fig 4.2) was chosen at the third position based on a previously performed structure calculation of the wildtype DNA with a thymidine at the modification site [Siepel2009]. The methyl group of the thymidine points out of the major groove and therefore its 
modification is less probable to induce a change in the secondary structure. The preservation of the secondary structure is an important condition for the usage of the concept of tagging for the determination of structural parameters. The modified nucleosides therefore are based on uracil with a modification at the 5 position (Fig,1.12).

During this work, two different approaches for tagging of DNA were investigated:

- Change of the linkage type of the Cys-Ph-TAHA tag.

First approaches with an EDTA-based tag in our group used a 1,3-dipolar cycloaddition (Huisgen reaction [Huisgen1963] [Gierlich2006]) of an azide and a carbon triple bond to connect tag and DNA via a triazole [Woeltjen2009] [Siepel2009]. Based on this work, the possibility to alter the Cys-Ph-TAHA tag linkage to a triazolyl moiety was investigated.

- Introduction of a thiole moiety into the DNA.

Since many known tags use disulfide bridges as a connection, introduction of a thiole group provides the opportunity to use different tags.

\subsection{Alteration Of The Linkage Type Of Cys-Ph-TAHA}

The 1,3-dipolar cycloaddition is a copper(I) catalyzed reaction. Its catalytic cycle is shown in Fig, 4.3 [Himo2005].

In the catalytic cycle, the first step is the addition of the alkyne to the copper-ligand complex by elimination of the terminal proton. Next, the azide binds to the copper via the negatively charged nitrogen. Subsequently, the first new carbon-nitrogen bond is formed. By elimination of the copper and formation of the second carbon-nitrogen bond, the intermediate six-membered ring is reduced to the triazole. The last step is the reductive elimination of the copper-ligand complex.

When using cycloaddition reactions on DNA, a crucial factor is the selection of the employed ligand. Oxidative scission of DNA strands catalyzed by the copper ion (Fenton reaction) reduces the yield of the reaction [Burrows1998]. Triazoyl compounds were first described by Chan et al. to stabilize the copper(I) species and to increase the reactivity for the cycloaddition, reducing the reaction time [Chan2004]. The reduction of the reaction time similarly reduces the time during which 


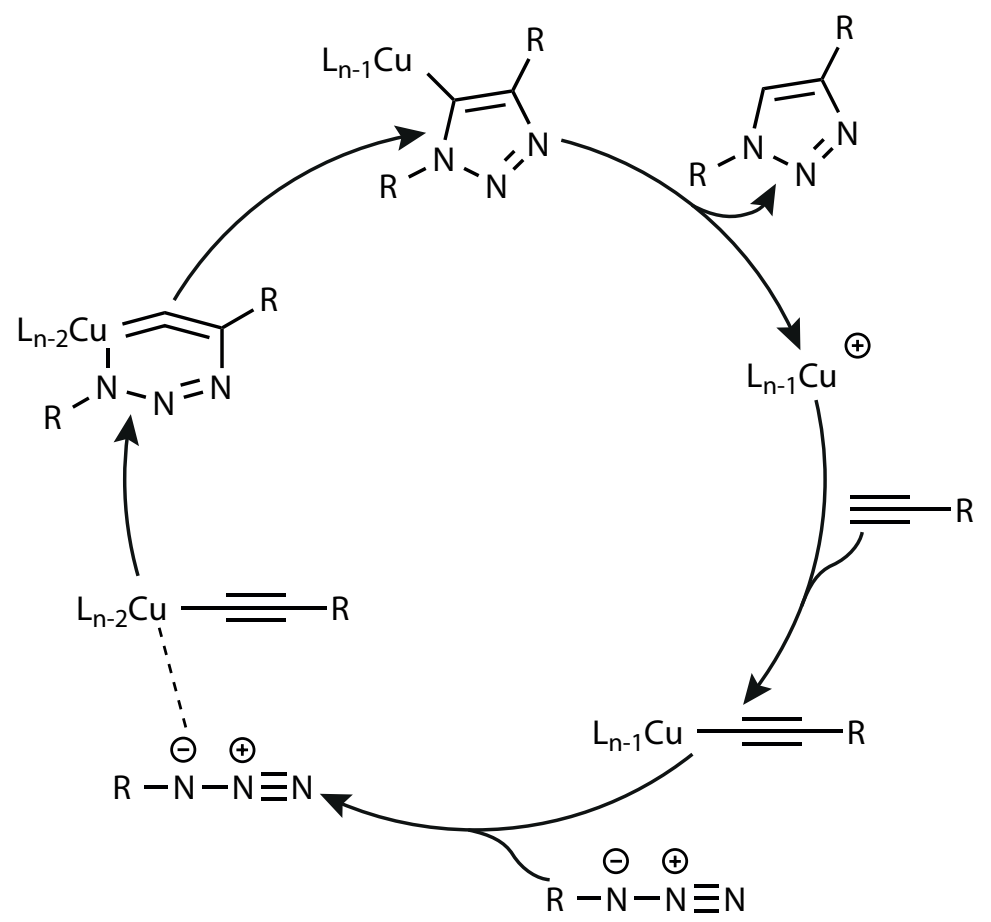

Figure 4.3: Catalytic cycle of the 1,3-dipolar cycloaddition.

the Fenton reaction can take place. In the previous work, the water-insoluble tris[(1-benzyl-1H1,2,3-triazol-4-yl)methyl]amine (TBTA) ligand was used for the cycloaddition and was also used in the first reactions of this work. To perform the reaction in aqueous solution, synthesis of the water soluble tris[[1-(3-hydroxypropyl)-1H-1,2,3-triazol-4-yl]methyl]amine (TPTA) ligand was performed (Fig:4.4) [Hein2011].

The 8 step synthesis of the modified nucleoside, containing a carbon triple bond, was described

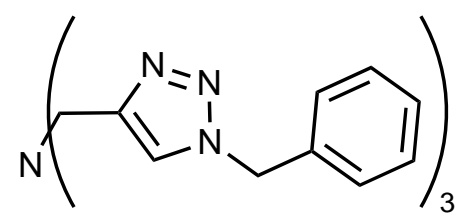

29

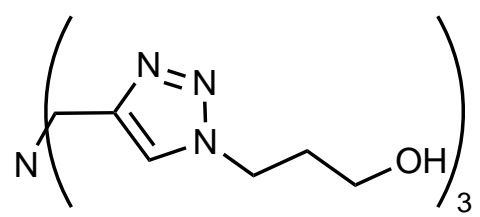

30 TPTA

Figure 4.4: The two triazolyl ligands used in this work. 
in a previous work [Woeltjen2009] [Siepel2009]. In this work, the synthetic route was optimized to 6 steps, significantly raising the overall yield from $20 \%$ to $49 \%$ (Fig:4.5). The starting molecule was 5-iodo-2'-desoxyuridine (30), which was in the first step protected at the $2^{\prime}$-position using a dimethoxy-trityl group. Two Sonogashira reactions were used to introduce the carbon triple bond [Sonogashira1975] [Sonogashira2002]. After each Sonogashira reaction, a deprotection step took place yielding 5'-O-dimethoxytrityl-5-[[(4-ethynyl)phenyl]ethynyl]-2'-desoxyuridine (34). The final nucleoside (35) for DNA synthesis was obtained by reaction of $\mathbf{3 4}$ with 2-cyanoethyl diisopropylchlorophosphoramidite.

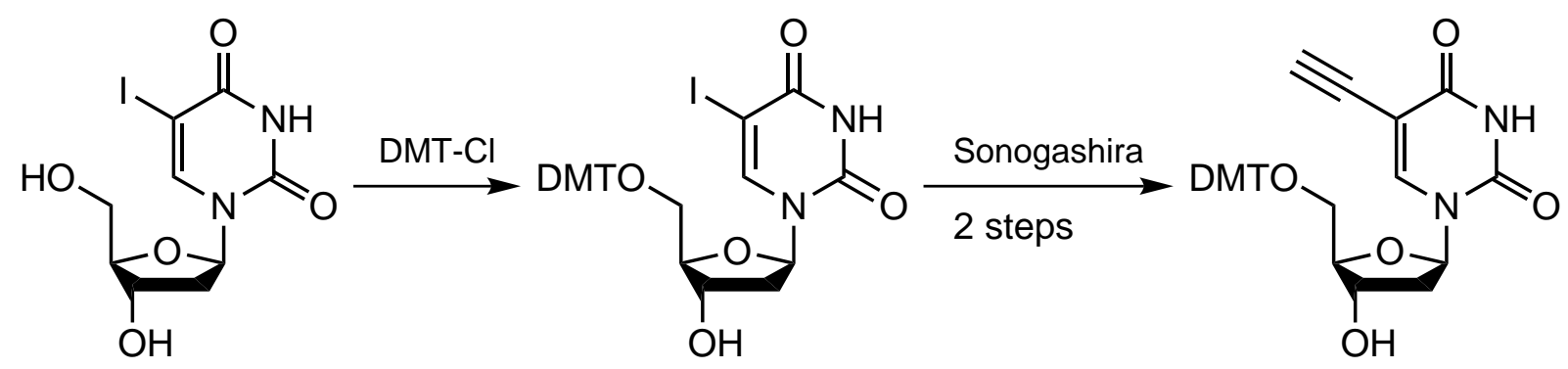

31

32

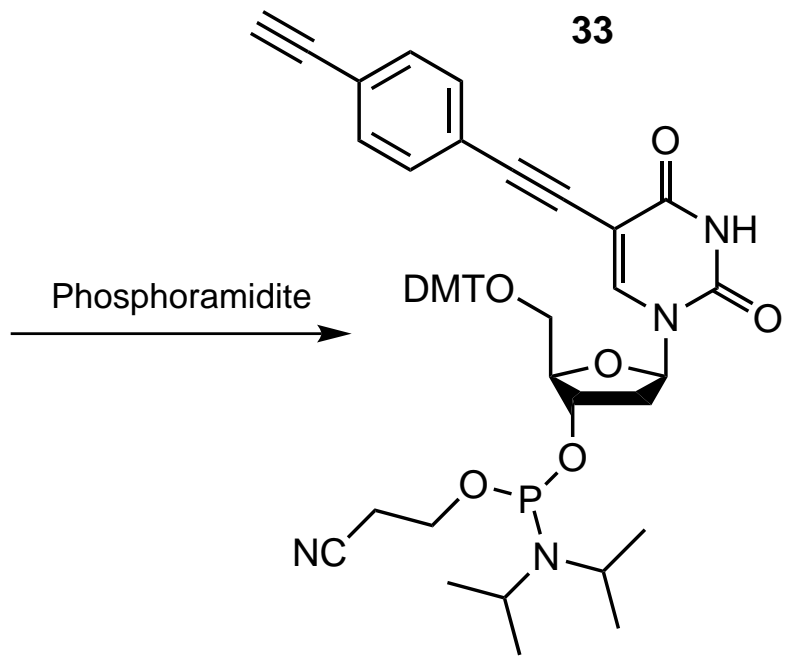

34

35

Figure 4.5: The reaction pathway to the final nucleoside containing a carbon triple bond.

For the introduction of an azide moiety into the tag, an intermediate product of the previously described Cys-Ph-TAHA synthesis was used [Peters2011]. 4-Bromo- $\alpha, \alpha, \alpha$-tris[[N,N-di(tert-butoxycarbonylmethyl)amino]methyl]toluene was converted to the corresponding azide by a copper(I) cat- 
alyzed substitution (Fig (4.6). The copper(I) species was stabilized by addition of trans- $N, N^{\prime}$-dimethylcyclohexane-1,2-diamine [Anderson2005].

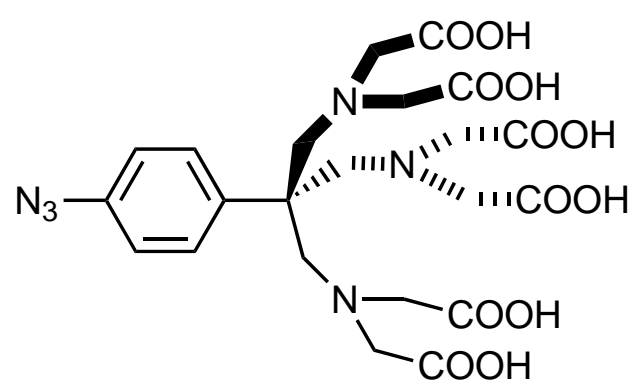

36

Figure 4.6: The modified tag for the cycloaddition, Azide-Ph-TAHA.

An overview of cycloaddition reactions performed with the new Azide-Ph-TAHA tag is given in Tab:2 The first reactions were performed with just a nucleoside and the lutetium loaded Azide-PhTAHA tag. As the tag can not be loaded quantitatively after the tagging reaction, a preloaded tag was used [Peters2011]. Several different copper(I) sources were examined. Copper bromide directly provides the correct oxidation state, whereas this state has to be generated in situ for solid copper and copper sulfate.

The following reactions were performed with phenylacetylene as a testmolecule, since it is commercially available and provides a carbon triple bond. The cycloaddition with DNA was tested next, first with TBTA and afterwards with the newly synthesized TPTA ligand. As is apparent from the table, the cycloaddition is unsuccessful when using the Azide-Ph-TAHA tag. The supposed reason for this is the chelating function of the tag which, even when already coordinating a lanthanide ion, is in competition to the ligand of the copper catalyst complex and prevents the formation of the catalytic species.

In order to investigate the presumed behaviour, NMR spectra of the Azide-Ph-TAHA tag loaded with diamagnetic lutetium were recorded (Fig:4.7). The two broad peaks at $2.98 \mathrm{ppm}$ and $2.86 \mathrm{ppm}$, with intensities of 2 and 4 , respectively, correspond to three $\mathrm{CH}_{2}$-groups. The four protons of the $\mathrm{CH}_{2}$-groups between the carboxyl groups and the nitrogen are equivalent, indicating that one arm is 
non-coordinating and in slow exchange with the other two. Therefore, the loaded tag does not exhibit C3-symmetry contrary to previous assumptions [Peters2011]. Two dimensional ${ }^{1} \mathrm{H}-{ }^{13} \mathrm{C}-\mathrm{HSQC}-$ spectra were recorded to analyze the coordination motif of the two chelating arms (Fig:4.8). In the attained spectrum, there are three sets of $\mathrm{CH}_{2}$-groups, each set with a combined intensity of four, corresponding to two equivalent groups. Therefore, the two arms bind equally to the lanthanide, but the two carboxyl groups of a single arm bind in different fashions. 


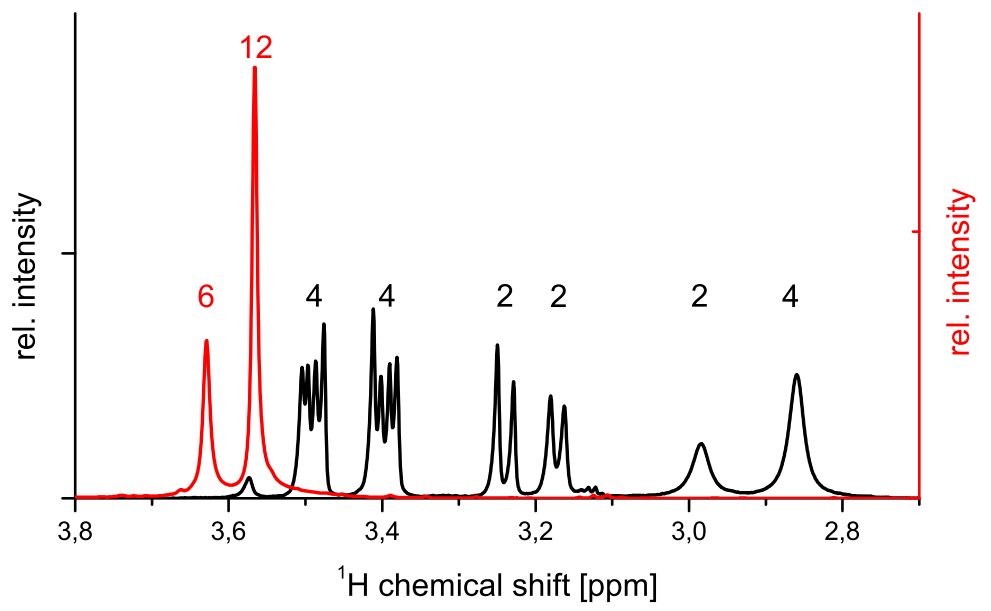

Figure 4.7: Overlay of the spectra for the lutetium loaded Azide-Ph-TAHA tag (black) and the unloaded tag (red). The numbers above the signals indicate the relative integral intensities.

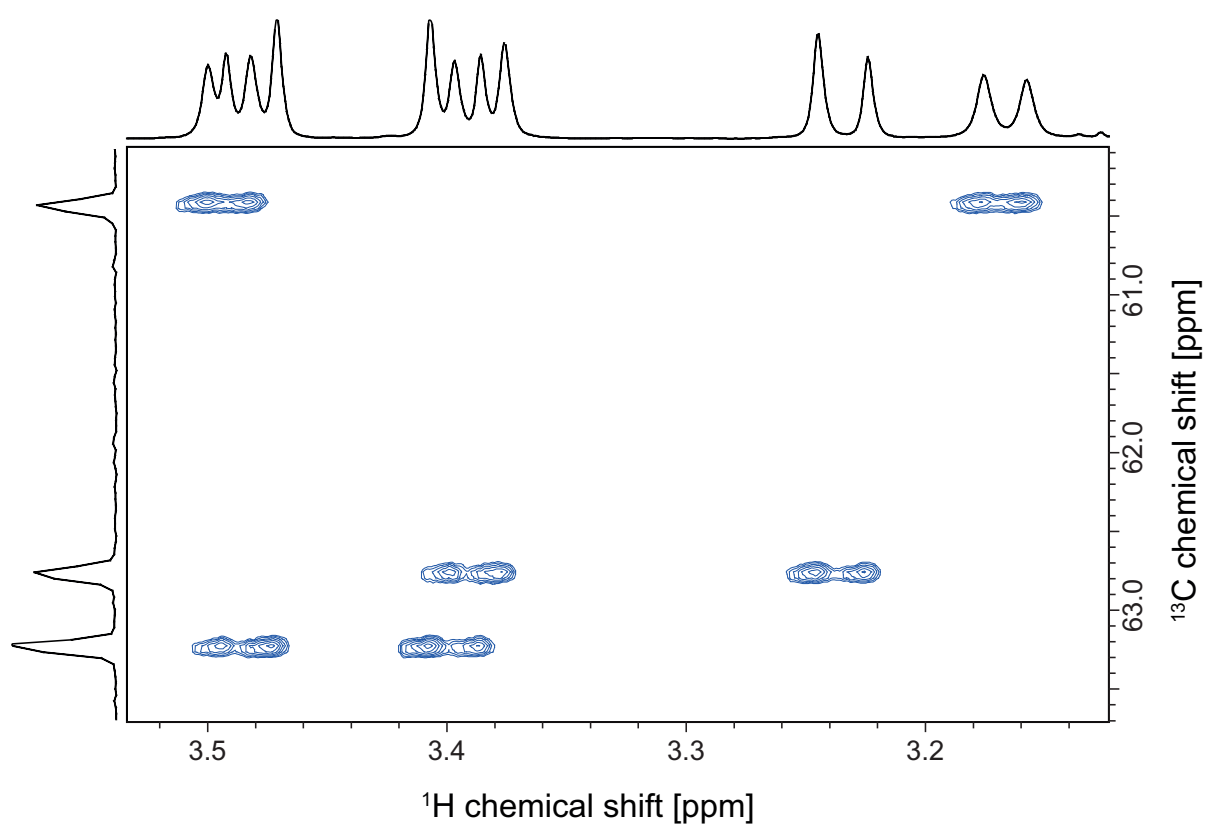

Figure 4.8: Section of the ${ }^{1} \mathrm{H}-{ }^{13} \mathrm{C}-\mathrm{HSQC}$ spectrum of the lutetium loaded tag. 


\begin{tabular}{|c|c|c|c|c|}
\hline Carbon triple bond molecule & Catalyst system & Ligand & Solvent & Product \\
\hline 33 & $\mathrm{CuBr}$ & TBTA & ${ }^{t} \mathrm{BuOH} \& \mathrm{H}_{2} \mathrm{O}$ & No \\
\hline 33 & $\mathrm{CuBr} \& \mathrm{NaAsc}$ & TBTA & ${ }^{t} \mathrm{BuOH} \& \mathrm{H}_{2} \mathrm{O}$ & No \\
\hline Phenylacetylene & $\mathrm{CuSO}_{4} \& \mathrm{NaAsc}$ & TBTA & ${ }^{t} \mathrm{BuOH} \& \mathrm{H}_{2} \mathrm{O}$ & No \\
\hline Phenylacetylene & $\mathrm{Cu}(\mathrm{s})$ & TBTA & ${ }^{t} \mathrm{BuOH} \& \mathrm{H}_{2} \mathrm{O}$ & No \\
\hline Phenylacetylene & $\mathrm{CuBr} \& \mathrm{NaAsc}$ & TBTA & ${ }^{t} \mathrm{BuOH} \& \mathrm{H}_{2} \mathrm{O}$ & No \\
\hline Phenylacetylene & $\mathrm{CuBr} \& \mathrm{NaAsc}$ & TBTA & ${ }^{t} \mathrm{BuOH} \& \mathrm{H}_{2} \mathrm{O} \& \mathrm{DMSO}$ & No \\
\hline Phenylacetylene & $\mathrm{CuSO}_{4} \& \mathrm{Cu}(\mathrm{s})$ & - & $\mathrm{H}_{2} \mathrm{O}$ & No \\
\hline DNA & $\mathrm{CuBr} \& \mathrm{NaAsc}$ & TBTA & ${ }^{t} \mathrm{BuOH} \& \mathrm{H}_{2} \mathrm{O} \& \mathrm{DMSO}$ & No \\
\hline DNA & $\mathrm{CuBr} \& \mathrm{NaAsc}$ & TBTA & ${ }^{t} \mathrm{BuOH} \&$ TEAA $(50 \mathrm{mmol} / \mathrm{L}) \&$ DMSO & No \\
\hline DNA & $\mathrm{CuSO}_{4} \& \mathrm{NaAsc}$ & TPTA & $\mathrm{H}_{2} \mathrm{O}$ & No \\
\hline DNA & $\mathrm{CuSO}_{4} \& \mathrm{NaAsc}$ & TPTA & $\mathrm{AA}(50 \mathrm{mmol} / \mathrm{L})$ & No \\
\hline 34 & $\mathrm{CuAc}_{2} \& \mathrm{NaAsc}$ & TPTA & $\mathrm{H}_{2} \mathrm{O} \& \mathrm{MeCN}$ & No \\
\hline
\end{tabular}

Table 2: Overview of performed cycloaddition reactions with the Azide-Ph-TAHA tag. 



\subsection{Introduction Of A Sulfur Moiety Into DNA}

Based on the synthetically modified nucleoside (Fig, 4.5) and the feasibility to perform cycloaddition reactions with the modified DNA strand, the introduction of a sulfur moiety via a cycloaddition between an azide and a carbon triple bond was investigated. The commercially available dithiobis(phenylazide) (DTBPA) was selected as a sulfur source (38, Fig:4.9], as this molecule provides an azide moiety and the desired sulfur atom is already covalently bound, thereby avoiding a poisoning of the catalyst.

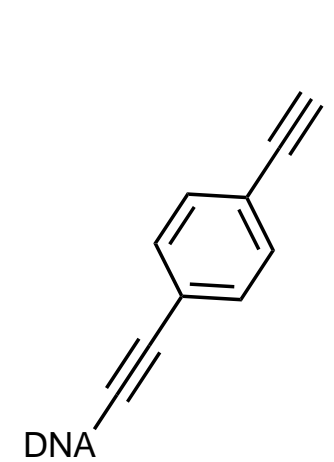

37

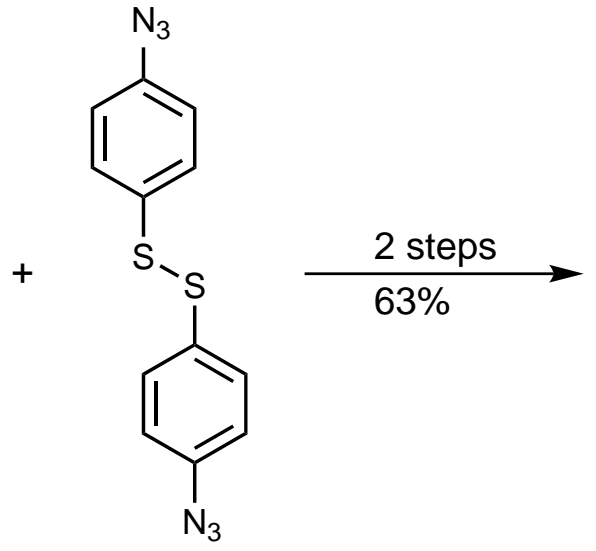

38

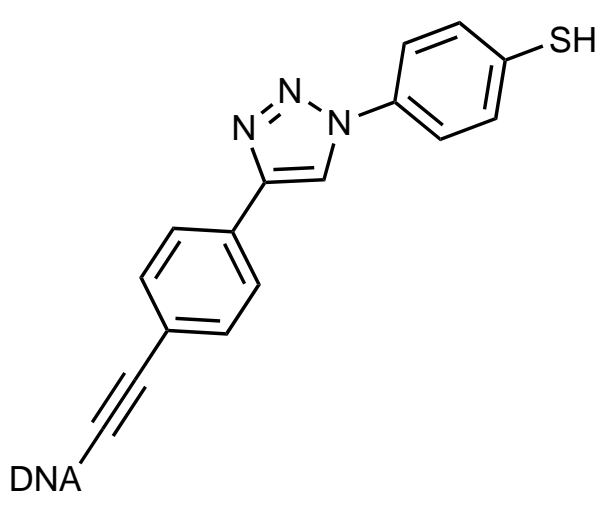

39

Figure 4.9: Cycloaddition to introduce a sulfur moiety into a DNA strand.

The conditions for this reaction were optimized to a yield of $63 \%$. The catalytic copper species was generated in situ by reduction of copper(II) acetate with sodium ascorbate. DTBPA is water insoluble and therefore a mixture of THF, MeCN and water was used as solvent. Application of the newly synthesized TPTA ligand was found to give higher yields than TBTA. The described catalyst/ligand system was also testet unsuccessfully on the cycloaddition of the Azide-Ph-TAHA tag (Tab:2, last entry), supporting the thesis that the TAHA group prevents the formation of the active catalytic species. Incubation of the modified DNA (39) with preloaded Cys-Ph-TAHA tag yielded the first DNA tagged with a lanthanide (Fig:4.10).

The combination of the modified nucleoside, dithiobis(phenylazide) and the Cys-Ph-TAHA tag results in a large distance between the lanthanide and the DNA strand (approximately $32 \AA$, measured 


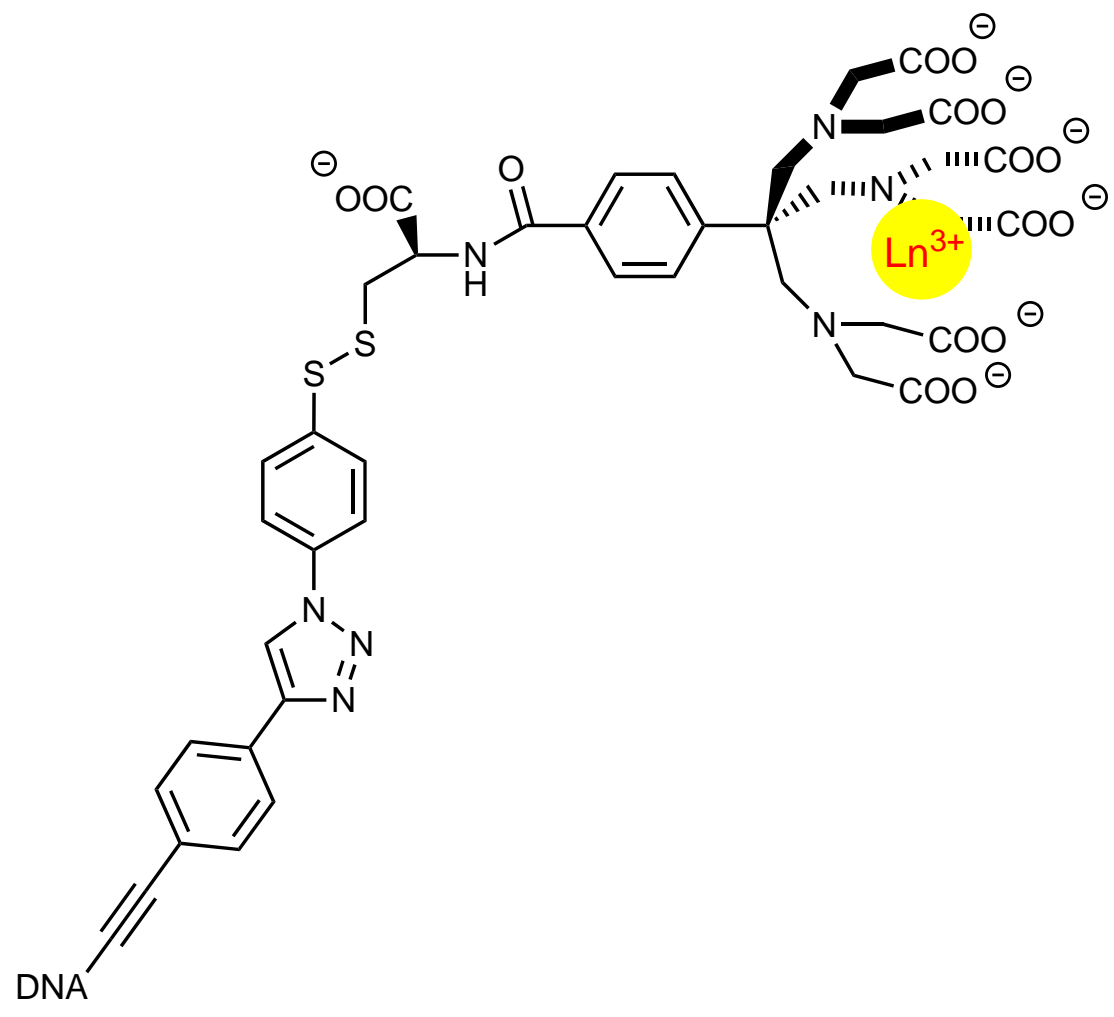

Figure 4.10: Structure of the first Cys-Ph-TAHA tagged DNA loaded with a lanthanide ion.

with Avogadro 1.0.3). This distance and the flexibility of the linker induce a high mobility of the lanthanide, which reduces the alignment and the observable effects in the NMR spectra. Therefore, a shorter and more rigid linker is preferred. 


\subsection{Shortening Of The Linker Of The Cys-Ph-TAHA Tag}

Similar to the introduction of the azide group into the tag, 4-bromo- $\alpha, \alpha, \alpha$-tris $[[N, N$-di(tert-butoxy-carbonylmethyl)amino]methyl]toluene and the deprotected 4-bromo- $\alpha, \alpha, \alpha$-tris[[N,N-di(carboxymethyl)-amino]methyl]toluene were used with the aim to substitute the bromine with a sulfur atom (Fig,4.11). Several different catalysts and sulfur sources were tried for this reaction (Tab:3]). The Pd-PEPPSI-IPent catalyst was described by Sayah \& Organ [Sayah2011] and was used on bulky bromide substrates, achieving high yields in the conversion to the sulfur product. However, the Pd-PEPPSI-IPent catalyst showed no conversion for 4-bromo- $\alpha, \alpha, \alpha$-tris[[ $N, N$-di $($ tert-butoxycarbonylmethyl)amino]methyl]toluene. In order to test if the tert-butoxy protection groups are too bulky, the same reaction was performed with the deprotected 4-bromo- $\alpha, \alpha, \alpha$-tris [[N,N-di(carboxymethyl)amino]methyl]toluene, without success. Yi et al. described the reaction of aromatic bromine compounds with sodium thiosulfate, tris(dibenzylideneacetone)dipalladium $\left(\operatorname{Pd}_{2}(\mathrm{dba})_{3}\right)$ and 2-dicyclohexyl-phosphino-2',4',6'-tri- $i$-propyl-1,1'-biphenyl (Xphos) [Yi2011]. This reaction also yielded no conversion. Another carbon-sulfur bond formation reaction was described by Forbes \& Zondlo [Forbes2012]. The sulfur source in this $\mathrm{Cu}(\mathrm{I})$ catalyzed reaction is thioacetic acid and 1,10-phenantroline was added as copper stabilizing ligand. Just as the other attempts, no conversion to the product was observed in this reaction.

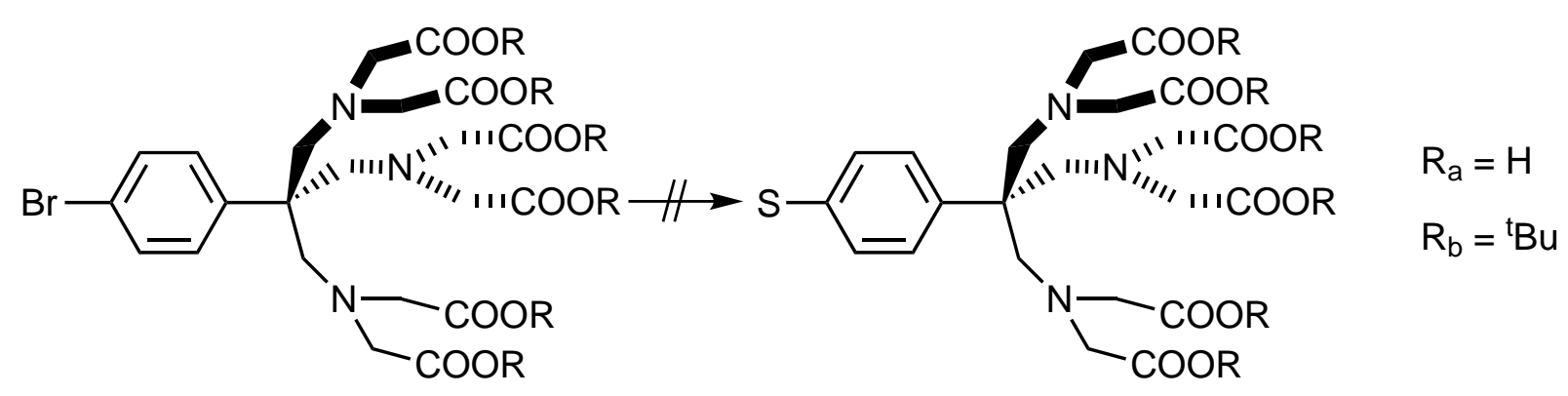

40a,b

41a,b

Figure 4.11: The tested exchange of the bromine with a sulfur.

Unfortunately, as Tab3 shows, the substitution of the aromatic bromine with a sulfur moiety was unsuccessful with the catalyst systems described in the literature. Compared to the successful substitution with an azide (Fig:4.6), the catalyst systems for the sulfur exchange reaction are much larger 
and, due to the big ligands, more sterically demanding. Therefore, the presumed reason for the unsuccessful reaction lies in the bulky carboxyl groups, which prevent a spatial approach of the catalyst and the bromine substrate. Consequently, the sulfur has to be introduced at an earlier stage of the synthesis route. This is still an ongoing project in our group. 
Educt: 4-bromo- $\alpha$ - $\alpha$ - $\alpha$-tris(( $N, N$-di(tert-butoxy-carbonylmethyl)amino)methyl)toluene

\begin{tabular}{c|c|c|c}
\hline Sulfur source & Catalyst system & Solvent & Product \\
\hline Triphenylmethanethiol & Pd-PEPPSI-iPent & Toluene & No \\
\hline${ }^{t}$ BuSH & Pd-PEPPSI-IPent & Toluene & No \\
\hline Thioacetic acid & CuI \& 1,10-Phenanthroline & Toluene & No \\
\hline Thioacetic acid & Pd-PEPPSI-IPent & Toluene & No \\
\hline Potassium thioacetate & CuI \& NaAsc \& trans- $N, N^{\prime}$-dimethylcyclohexane-1,2-diamine & EtOH \& DMSO \& $\mathrm{H}_{2} \mathrm{O}$ & No \\
\hline Sodium thiosulfate & $\mathrm{Pd}_{2}(\mathrm{dba})_{3} \&$ Xphos \& Caesiumcarbonat & $\mathrm{H}_{2} \mathrm{O}$ & No \\
\hline
\end{tabular}

Educt: 4-bromo- $\alpha-\alpha-\alpha$-tris(( $N, N$-di(carboxymethyl)amino)methyl)toluene

\begin{tabular}{c|c|c|c}
\hline Sulfur source & Catalyst system & Solvent & Product \\
\hline Triphenylmethanethiol & Pd-PEPPSI-IPent & Toluene & No \\
\hline
\end{tabular}

Table 3: Overview of performed bromine-sulfur substitution reactions. 



\subsection{Results}

The introduction of an endstanding carbon-carbon triple bond and a subsequent cycloaddition reaction with DTBPA led to a modified DNA strand with a free thiol moiety. The product of each step in the synthesis of the tagged DNA was verified by mass spectrometry. In Fig:4.12, the mass spectra of the product of the cycloaddition (left) and of the subsequent deprotection to give the free thiol moiety (right) are shown.

The first prepared tagged DNA was a diamagnetic reference sample in which the tag was loaded with diamagnetic lutetium. Following the tagging protocol described by Peters et al., the tag was incubated with 1.2 eq. of the corresponding lanthanide solution for $2 \mathrm{~h}$ [Peters2011]. After adjusting the $\mathrm{pH}$ to 7 with $0.1 \mathrm{~mol} / \mathrm{L}$ sodium hydroxide solution, precipitated lanthanide hydroxide was removed by centrifugation. The supernatant was added to the DNA with the free thiol moiety and incubated for $12 \mathrm{~h}$. Subsequent HPLC purification yielded tagged DNA loaded with the corresponding lanthanide. The HPLC chromatogram is shown in the experimental details. The lutetium-loaded DNA
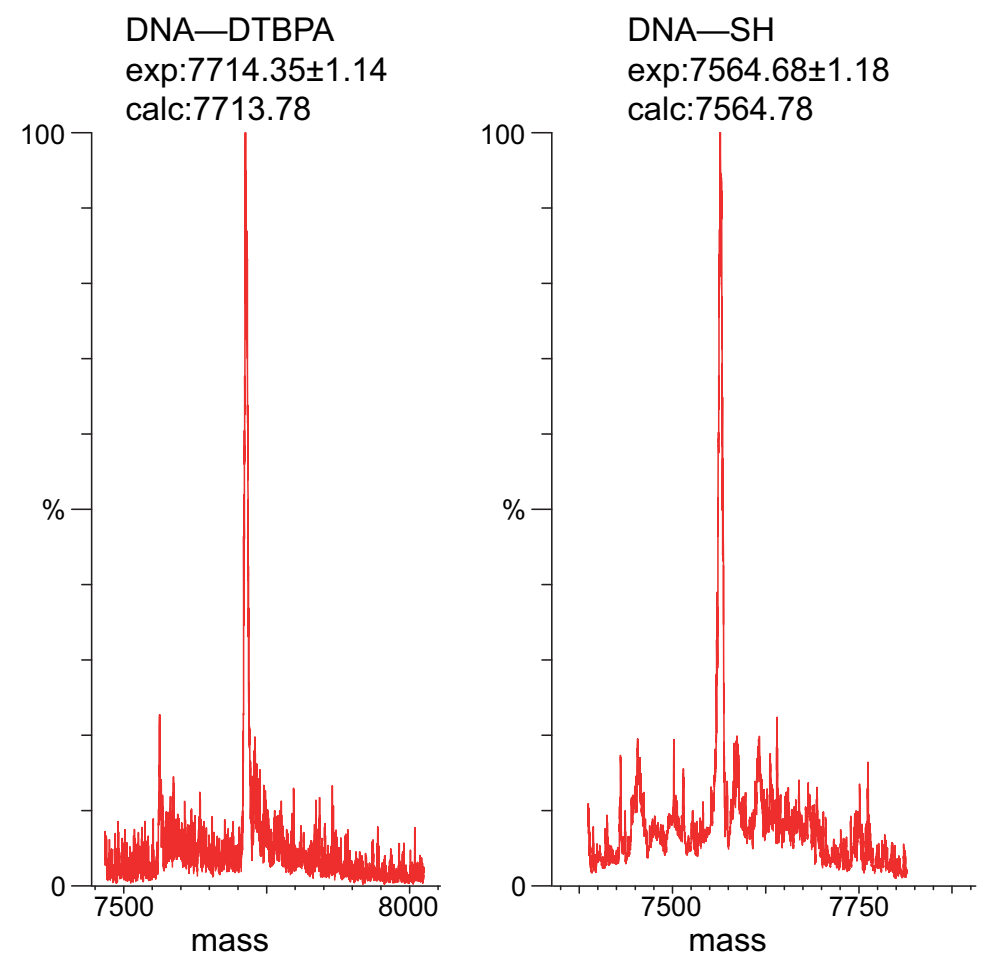

Figure 4.12: Mass spectra of the DNA strands with DTBPA (left) and with the free thiol moiety (right). 


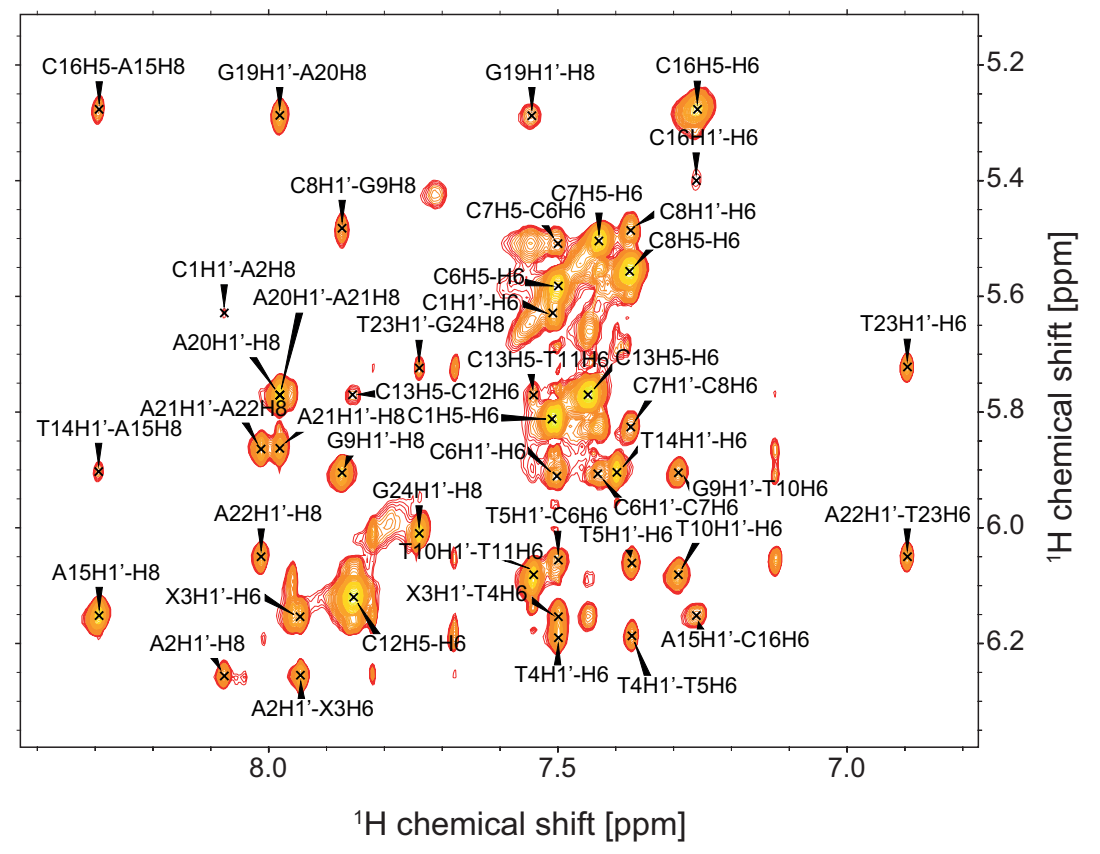

Figure 4.13: NOESY walk region of the ${ }^{1} \mathrm{H}-{ }^{1} \mathrm{H}$-NOESY spectrum of the lutetium loaded DNA.

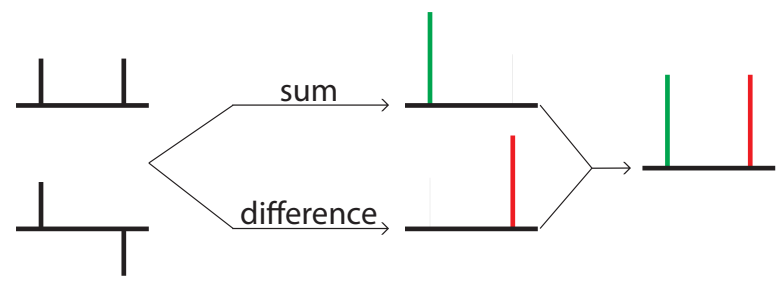

Figure 4.14: Schematic representation of the principle behind ${ }^{1} \mathrm{H}-{ }^{13} \mathrm{C}-\mathrm{HSQC}$-IPAP spectra.

was lyophilized, dissolved in $250 \mu \mathrm{L} \mathrm{D}_{2} \mathrm{O}$ with $2 \mathrm{mmol} / \mathrm{L}$ ammonium acetate $(\mathrm{pH} 7)$ and transferred into a Shigemi NMR tube. Unless otherwise stated, all NMR spectra were recorded at 298 K.

The one dimensional proton spectra showed the expected narrow line widths for a diamagnetic sample. Two dimensional ${ }^{1} \mathrm{H}-{ }^{1} \mathrm{H}-N O E S Y$ (Fig:4.13) and ${ }^{1} \mathrm{H}^{1}{ }^{1} \mathrm{H}-\mathrm{COSY}$ spectra were recorded to assign the peaks of the reference spectra.

Assignment of the aromatic signals was achieved using a NOESY walk [Roberts1993], leading to a complete assignment, except for the overlaid signals of adenosine $20-22$ and the loop region.

${ }^{1} \mathrm{H}_{-}{ }^{13} \mathrm{C}-\mathrm{HSQC}-\mathrm{IPAP}$ spectra were recorded to determine the $\mathrm{C}-\mathrm{H}$ coupling constants and for a completion of the reference spectra set. A schematic of the NMR experiment applied to record these 
spectra is given in Fig 4.14

Two proton-carbon correlated spectra were recorded in an interleaved method, one of which results in in-phase and the other in anti-phase magnetization. The sum and the difference of these two spectra are added up to give an in-phase spectrum. The obtained signal intensity is high, compared to regular HSQC spectra, since twice the number of experiments are performed due to the interleaved IPAP method, resulting in redoubled signal intensity. In standard NMR experiments, a doubling of the number of scans increases the signal intensity only by a factor of $\sqrt{2}$. The pulse program for this experiment was adopted from the corresponding ${ }^{1} \mathrm{H}-{ }^{15} \mathrm{~N}-\mathrm{HSQC}-$ IPAP pulse program [Ottiger1998a].

After the measurements of the reference spectra, samples with different lanthanides were prepared. The mass spectra of the three differently loaded DNA strands are shown in Fig:4.15, Following the same tagging protocol used to prepare the diamagnetic reference sample, a terbium loaded DNA sample was examined as the first paramagnetic sample. An overlay of the aromatic regions of the proton NMR spectra is shown in Fig:4.16. As easily inferred from the spectra, the resolution of the
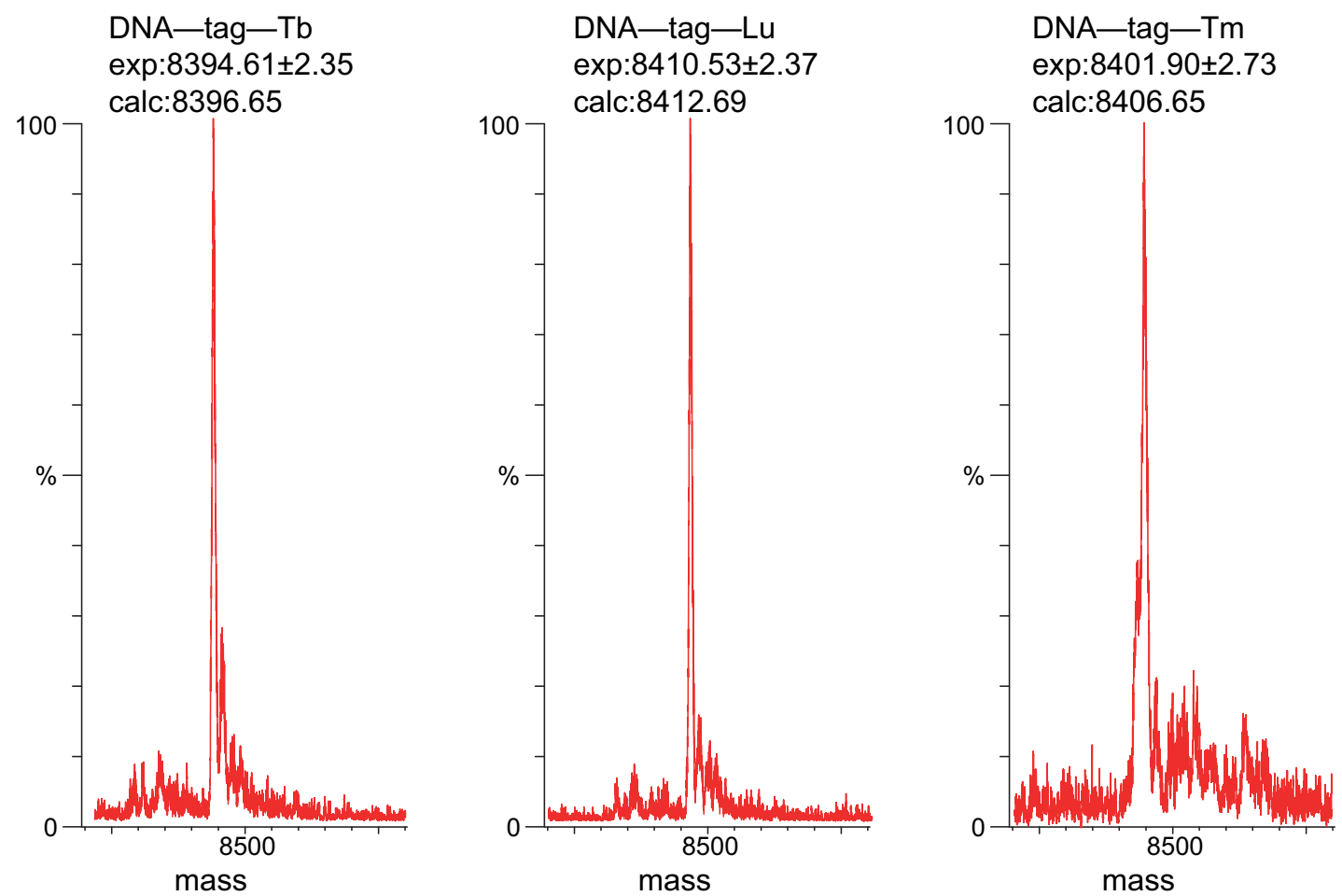

Figure 4.15: Mass spectra of the three tagged DNA strand: Tb (left), Lu (center) and Tm (right). 
paramagnetic sample is much smaller than for the diamagnetic one. The presumed reason for this paramagnetic linebroadening is an excess of the lanthanide, either bound to the phosphate backbone or in a water-soluble complex. Another effect which can induce line broadening is a misfolding of the DNA strand [Roberts1993].

A GE Healthcare PD-10 size exclusion column was used to remove the excess of lanthanide. Subsequently, the sample solution was heated to $65^{\circ} \mathrm{C}$ to unfold the DNA strand and afterwards cooled to ambient temperature to induce the proper folding. After this procedure, mass spectra showed only degradation products and no tagged DNA. As mentioned in the previous section, oxidative scission of DNA strands is catalyzed by metal ions, and the efficiency of this reaction might be increased at higher temperatures [Burrows1998]. The observation of this degradation implies that the lanthanide is bound to the phosphate backbone and that a size exclusion column is not suitable to remove the excess of lanthanide.

The loading of the tag for the next sample was performed with $1.1 \mathrm{eq}$. of Tb to reduce the excess of lanthanide. An overlay of different spectra of this sample is shown in Fig:4.17, The blue spectrum was recorded after the tagging reaction and one HPLC purification run $(\mathrm{MeCN} \& 50 \mathrm{mmol} / \mathrm{L}$ ammonium acetate). The resolution was increased compared to the previous $\mathrm{Tb}$ sample (Fig:4.16), but residual ammonium acetate buffer led to an immense signal at $1.92 \mathrm{ppm}$ with a 400 -fold intensity compared

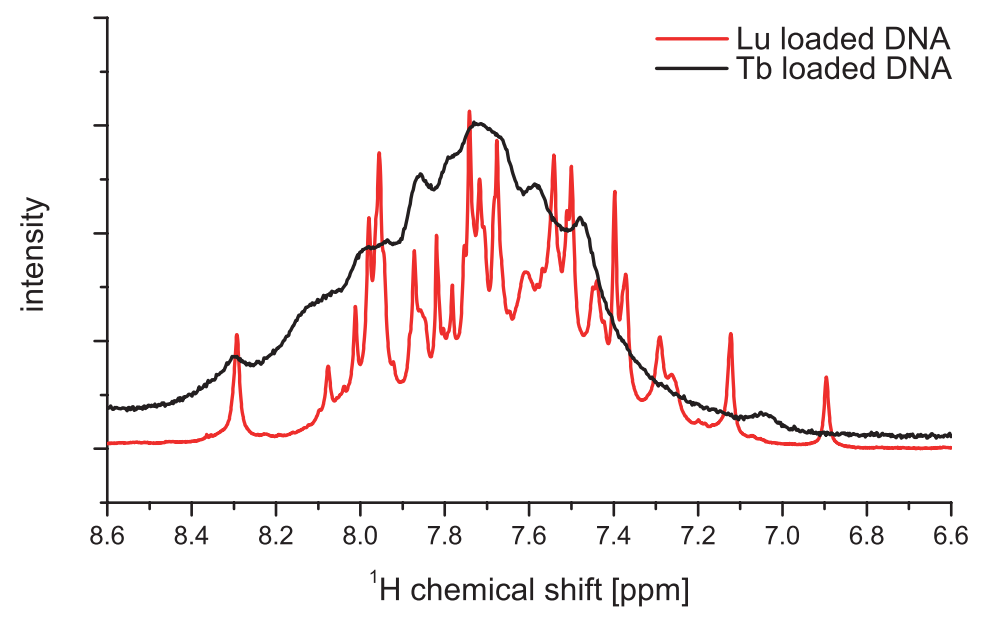

Figure 4.16: The aromatic region of diamagnetic (Lu: red) and paramagnetic (Tb: black) DNA. The spectra are scaled for comparability. 


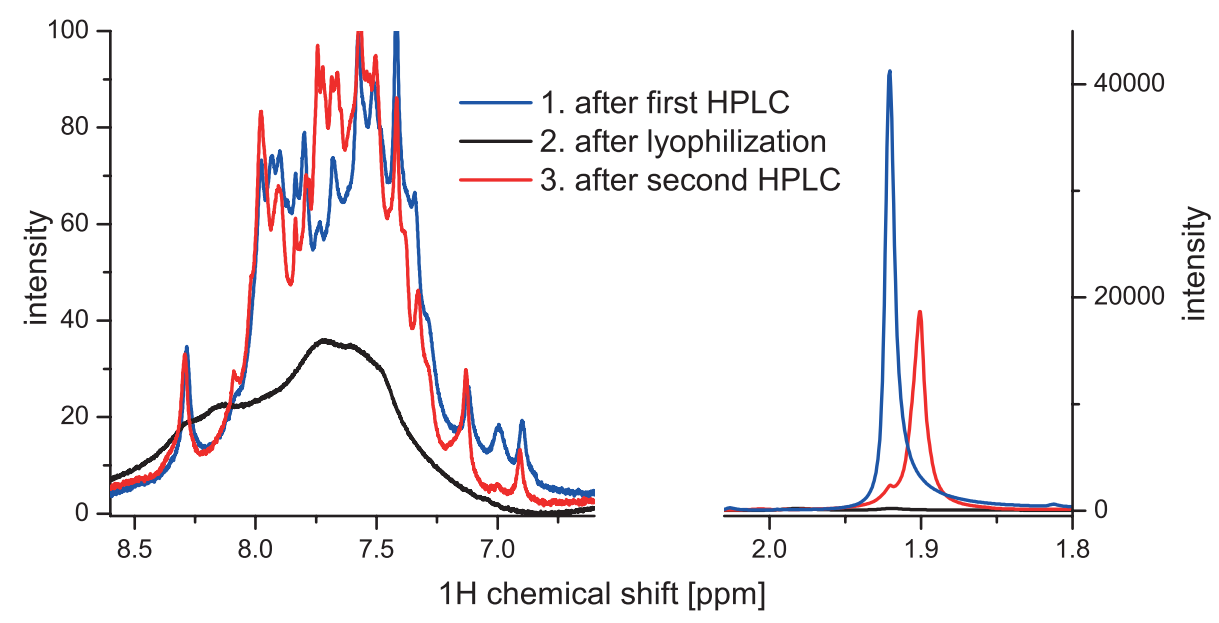

Figure 4.17: Aromatic region and ammonium acetate signal of Tb-loaded DNA. 1. Blue: After tagging and one HPLC purification run. 2. Black: After 1. and one week of lyophilization. 3. Red: After 2. and a second HPLC purification sun.

to the DNA signals. Subsequently, the buffer was removed by one week of lyophilization, resulting in the black spectrum with just one broad unresolved peak in the aromatic region of the spectrum. After a second HPLC purification run and re-addition of the buffer, a spectrum similar to the first one was recorded (red spectrum), although minor changes in the chemical shifts were observed.

There are three possible explanations for these observations:

1. The acetate ions form a complex with the excess of the lanthanide ions and prevent the coordination of the lanthanide ions to the phosphate backbone.

2. The ammonium ions bind to the phosphate backbone and block the possible binding sites for the lanthanide ions.

3. A combination of both effects.

Mass spectra of the sample showed tagged DNA with and without lanthanide, which explains the changes in the chemical shifts, mentioned above. Mixtures of loaded and non-loaded DNA strands led to a twin signal set in the NMR spectra, which is highly unfavorable. In a further attempt to remove the excess of lanthanide ions, the sample solution was dialysed against EDTA (0.1 mol/L) and ammonium acetate $(50 \mathrm{mmol} / \mathrm{L})$ solutions. Mass spectra showed that both dialysis procedures led to a complete loss of coordinated lanthanide ions, yielding only non-loaded DNA. This observation 
indicates that acetate ions, at least in high concentration, form a complex with the lanthanide ions and are able to completely remove it from the Cys-Ph-TAHA tag. This effect was not observed for 2 $\mathrm{mmol} / \mathrm{L}$ ammonium acetate concentrations.

To saturate the phosphate backbone with a different cation and to avoid the release of the lanthanide ions, the sample was washed using a Millipore Amicon $15 \mathrm{~mL}$ device with $3000 \mathrm{~g} / \mathrm{mol}$ molecular weight cut off and a sodium chloride solution $(12 \times 15 \mathrm{~mL})$. The effect of such a washing on a freshly prepared and HPLC purified Tb-sample is shown in Fig:4.18, As can be seen, the quality of

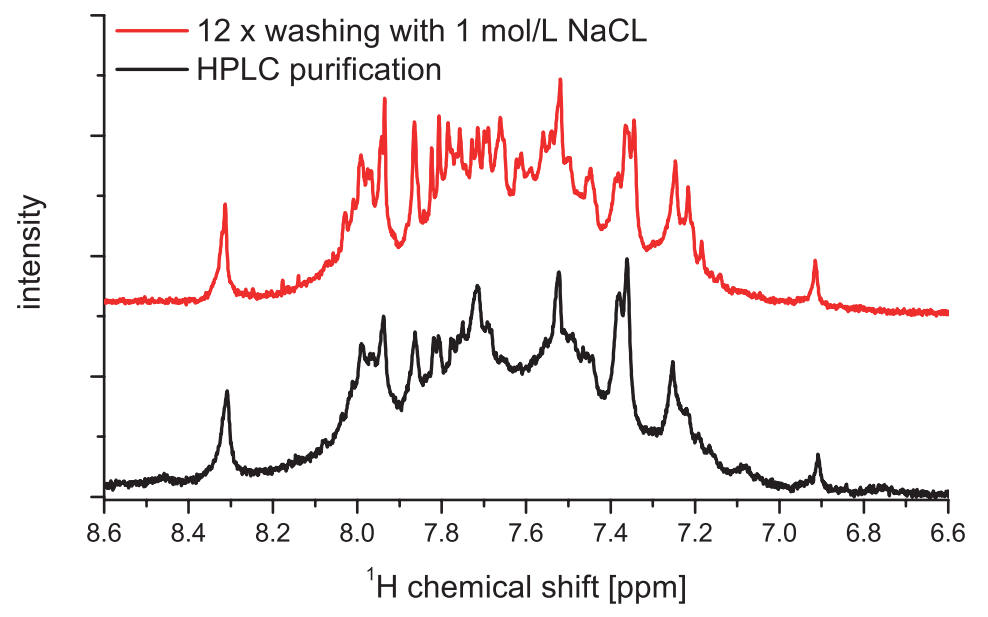

Figure 4.18: Aromatic region of Tb loaded DNA after HPLC purification (black) and after washing with $1 \mathrm{~mol} / \mathrm{L} \mathrm{NaCl}$ solution $(12 \times 20 \mathrm{~mL})($ red $)$.

the spectrum is significally improved, proving the efficiency of this method. Nevertheless, since the resolution was still inferior to the diamagnetic sample spectrum, 5 more washing cycles with $\mathrm{NaCl}$ were performed, followed by 2 cycles using $2 \mathrm{mmol} / \mathrm{L}$ ammonium acetate solution to remove $\mathrm{NaCl}$. Subsequently, the sample was lyophilized, the dried DNA dissolved in $\mathrm{D}_{2} \mathrm{O}$ and an NMR spectrum immediately recorded (Fig 4.19 blue). The observed resolution of the spectrum was comparable to the diamagnetic sample, though after $3.5 \mathrm{~h}$ a slight decrease in quality was observed (red). After $28.5 \mathrm{~h}$, the quality had significantly decreased to a level comparable to earlier samples, in which the lanthanide ions were coordinated to the phosphate backbone (green). $51 \mathrm{~h}$ after the sample preparation (orange), mass spectra showed only non-loaded tagged DNA, indicating that a removal of $\mathrm{NaCl}$ leads to a complete release of the lanthanide ions from the tag. 


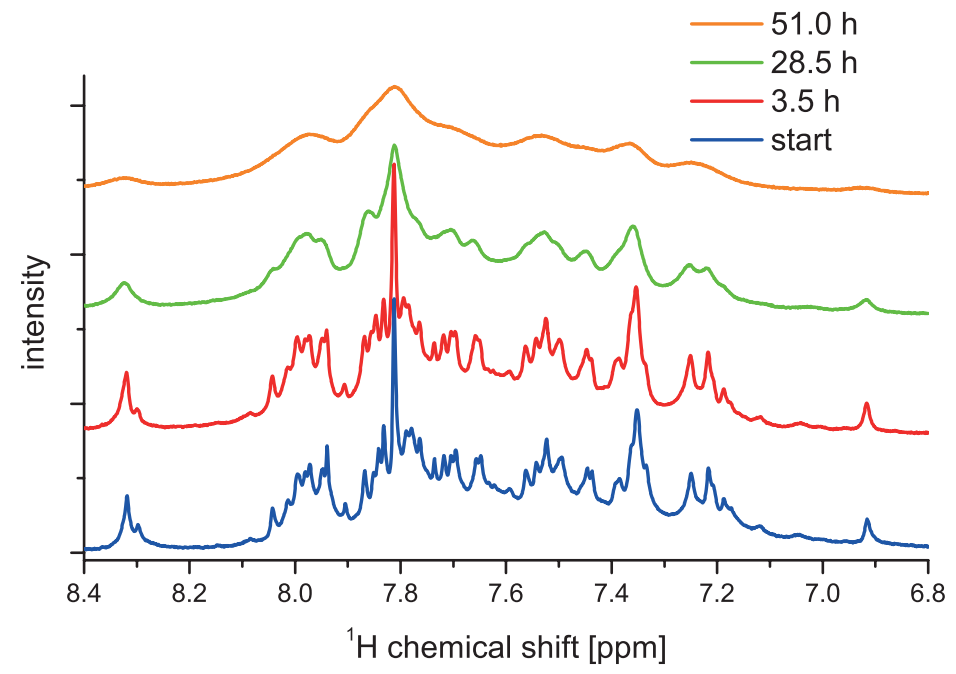

Figure 4.19: Aromatic region of Tb loaded DNA several times after preparation of the sample.

The most recent results show that adjusting the $\mathrm{NaCl}$ concentration of the sample to approximately $200 \mathrm{mmol} / \mathrm{L}$ and using MOPS buffer (3-morpholinopropane-1-sulfonic acid) (10 mmol/L, $\mathrm{pH} 7.5$ ) results in stable samples and gives reproducible high resolution spectra (internal communication with Sebastian Täubert).

\subsection{Discussion \& Outlook}

In this work, several approaches to adopt the concept of protein tagging to DNA molecules were investigated. A synthetic modification of the Cys-Ph-TAHA tag, in order for it to bind via formation of a triazole moiety, yielded the Azide-Ph-TAHA tag. Even though cycloaddition reactions between DOTA-based tags and DNA molecules for electron spin resonance spectroscopy were already reported [Song2011], the attachment site in that approach was at the $5^{\prime}$-end with the least sterical demand and the highest flexibility, which is highly undesirable for inducing paramagnetic effects. For the Azide-Ph-TAHA tag, no successful cycloaddition reaction could be mediated under various applied conditions. Nevertheless, the possibility to attach a DOTA-based tag to the modified DNA strand used in this work will be further investigated in the future.

Combination of the 1,3-dipolar cycloaddition and the disulfide binding motif of the Cys-Ph-TAHA tag led to the first metal-tagged DNA strand for NMR spectroscopy (Fig.4.20). Preparation of a 


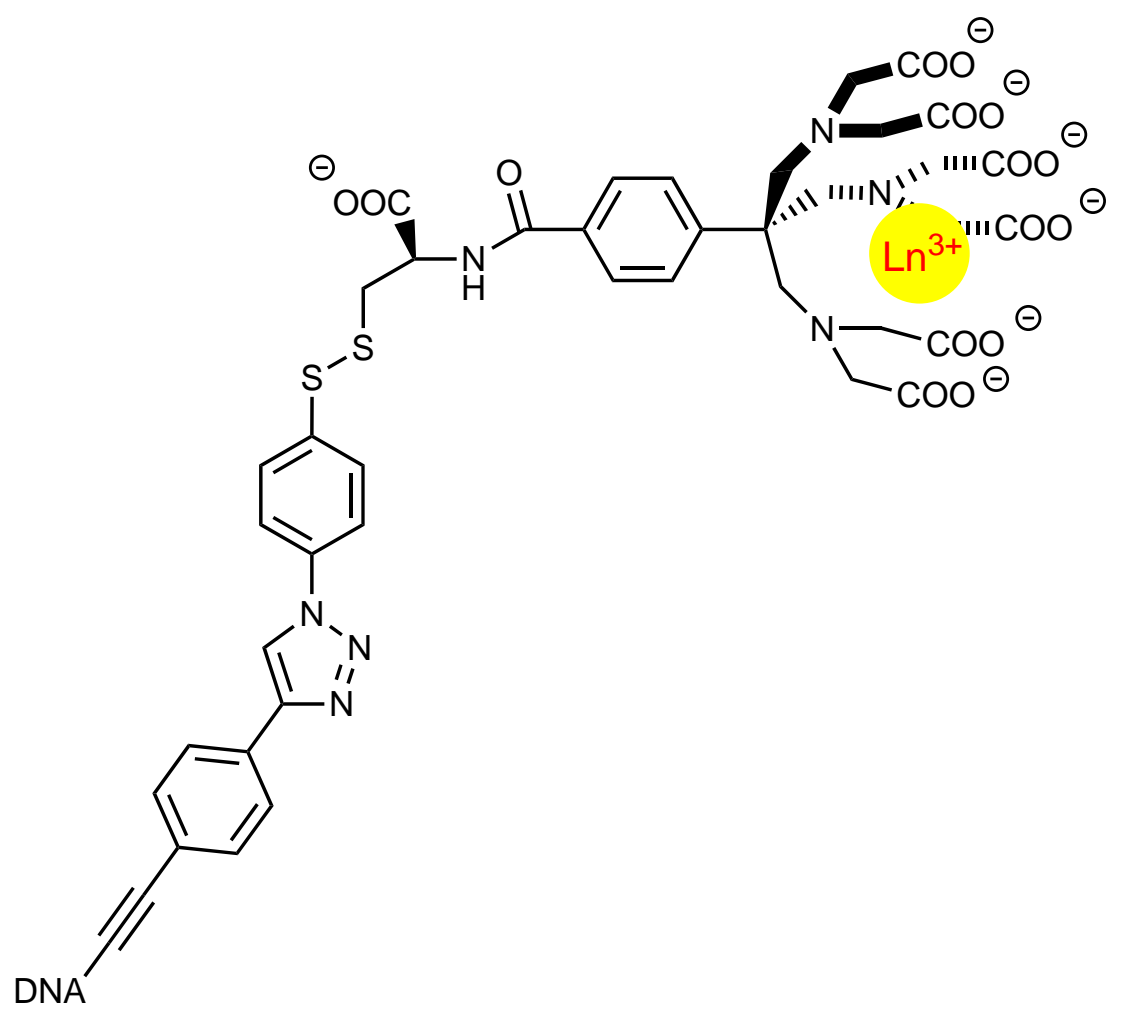

Figure 4.20: Combination of disulfide binding and cycloaddition yielded Cys-Ph-TAHA-tagged DNA.

diamagnetic reference sample, quantitatively loaded with lutetium was straightforward, following the tagging protocols for proteins. Two dimensional high resolution ${ }^{1} \mathrm{H}_{-}{ }^{1} \mathrm{H}-\mathrm{NOESY},{ }^{1} \mathrm{H}-{ }^{1} \mathrm{H}-\mathrm{COSY}$ and ${ }^{1} \mathrm{H}-{ }^{13} \mathrm{C}-\mathrm{HSQC}-$ IPAP spectra were recorded. During the preparation of the paramagnetic samples the crucial point of tagging DNA strands became apparent, namely the chelating property of the phosphate backbone. Time- and sample-consuming optimizations of the tagging protocol revealed the following aspects which have to be dealt with:

- Any excess of lanthanide is efficiently bound to the backbone.

- EDTA, phosphate buffer and acetate buffer (in concentrations above $2 \mathrm{mmol} / \mathrm{L}$ ) lead to a release of the lanthanide ions from the tag.

- Washing the sample with $\mathrm{NaCl}$ solution prevents the binding of lanthanide ions to the backbone, yet a backbone unsatured in regard of ion coordination (without $\mathrm{NaCl}$ ) competes with the tag for the lanthanide ions and also leads to a release of the lanthanide ions.

Considering these observations, very recently a promising purification protocol was developed. 
Adjusting the $\mathrm{pH}$ with MOPS-buffer, washing several times with $\mathrm{NaCl}$-solution $(20 \times 15 \mathrm{~mL})$ and providing a constant $\mathrm{NaCl}$ concentration of at least $200 \mathrm{mmol} / \mathrm{L}$ allowed for repeated measurements of identical NMR spectra with a high resolution (data by Sebastian Täubert). To obtain reliable reference spectra, a diamagnetic reference sample has to be prepared, following the new protocol, and all of the reference spectra have to be re-recorded.

\subsubsection{Concluding Remarks}

A method to successfully introduce a solvent exposed sulfur moiety to a modified DNA strand was developed. In principle, this method provides the potential to attach each disulfide-binding-based tag to the DNA, which was demonstrated using preloaded Cys-Ph-TAHA tag. The next steps for this project include the preparation of a diamagnetic reference sample, investigation and analysis of the induced paramagnetic effects and the transfer of the technique to different systems.

The first planned application of the tagged DNA is shown in Fig:4.21 The test molecule is the bulge and loop region of the trans-activation response element (TAR-RNA) of the human immunodeficiency virus-1 (HIV-1), which is essential for the virus replication and therefore a major drug targeting motif. The group of Al-Hashimi used an elongation of 22 base pairs, ${ }^{13} \mathrm{C}$ - and ${ }^{15} \mathrm{~N}$-labeling and phages

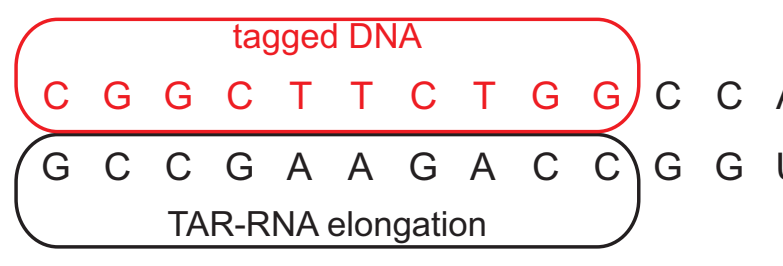

TAR-RNA loop and bulge of HIV-1

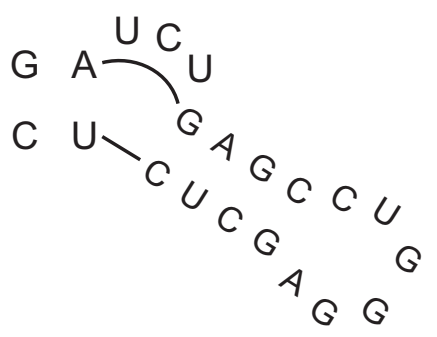

Figure 4.21: Elongation and binding of tagged DNA to TAR-RNA of HIV-1.

as orientation media to measure RDCs. This method provided new insights and revealed nano-tomillisecond domain motions upon ligand binding [Zhang2006] [Zhang2007]. A significantly shorter elongation of the RNA molecule provides a binding site for a complementary DNA strand. If this DNA strand is modified and tagged, all paramagnetic effects will be introduced to the RNA strand, 
without the need for labeling or orientation media. For the presented modified nucleotide, all cytidine residues in the DNA strand are possible tagging sites. Therefore, paramagnetic tagging of DNA and RNA strands provides a very useful tool for gathering structural and dynamic information. The methods and molecules developed and synthesized during this work make it possible to apply this tool to highly intriguing and challenging molecules of popular interest. 


\section{Experimental Part}

\subsection{Materials}

All solvents were purchased in pro analysis quality from Merck and Fluka. Argon was used as shielding gas for oxygen and moisture sensitive reactions and was dried over phosphorus pentoxide. All chemicals were purchased from Merck, Fluka, Alfa Aesar, Sigma-Aldrich, Acros and Apollo.

\subsection{Methods}

Flash column chromatography was performed with silica gel from Merck with a particle size of 63-100 $\mu \mathrm{m}$ and pressures between 1.0 and 1.5 bar. The crude product was applied as a concentrated solution in the elution solvents. Reaction control was performed with thin layer chromatography and phoshpomolybdic acid solution in ethanol (10\%) and iodine were used as coloring substances. Precoated silica gel SIL G/UV254 plates from Merck were used for thin layer chromatography.

\subsection{Analysis}

\subsubsection{Nuclear Magnetic Resonance Spectroscopy}

All NMR spectra for analysis of the synthetic molecules were recorded on a Bruker Avance U1trashield Spectrometer (400 MHz) at $298 \mathrm{~K}$. The used solvents are given in the experimental details. The chemical shifts are given in parts per million (ppm) in relation to the residual solvent signal.

\begin{tabular}{c|c|c} 
solvent & \multicolumn{2}{|c}{ chemical shift (ppm) } \\
\hline & ${ }^{1} \mathrm{H}$ & ${ }^{13} \mathrm{C}$ \\
\hline $\mathrm{CDCl}_{3}$ & 7.26 & 77.0 \\
\hline DMSO-d6 & 2.49 & 39.7
\end{tabular}

Following notation for the signals is used: chemical shift $\delta$ in ppm, multiplicity $(\mathrm{s}=$ singlet, $\mathrm{d}=$ doublet, $\mathrm{t}=$ triplet, $\mathrm{m}=$ multiplet $)$, scalar coupling constant $J$ in $\mathrm{Hz}$, intensity and nucleus. Assignment of the signals was achieved with two dimensional $\left[{ }^{1} \mathrm{H},{ }^{13} \mathrm{C}\right]-\mathrm{HSQC}$ and $\left[{ }^{1} \mathrm{H},{ }^{13} \mathrm{C}\right]-\mathrm{HMBC}$ spectra. 


\subsubsection{HPLC Chromatography}

HPLC purification was performed on Jasco systems equipped with a multiwavelength detector. Reversed phase Knauer Eurospher C18 columns $(250 \times 8 \mathrm{~mm})$ were used. A gradient of triethylammonium acetate buffer in water $(0.1 \mathrm{~mol} / \mathrm{L})$ and acetonitrile was used as eluent. The gradient and eluation speed are given in the experimental details.

\subsubsection{Mass Spectrometry}

The mass spectra were measured on a Waters electron spray ionisation mass spectrometer (Micromass ZQ) with quadropole detector. The values are given in mass per charge $(\mathrm{m} / \mathrm{z})$ and the used solvents are given in the experimental details.

\subsubsection{UV/Vis-Spectroscopy}

Spectra were recorded using a UV/Vis-spectrometer 8453 from Hewlett Packard.

\subsection{DNA}

Synthesis of the wildtype DNA and the modified DNA were performed by IBA. 


\subsection{Experimental Details}

\subsubsection{5'-O-Dimethoxytrityl-5-iodo-2'-desoxyuridine}<smiles>CC(O)n1cc(I)c(=O)[nH]c1=O</smiles>

31

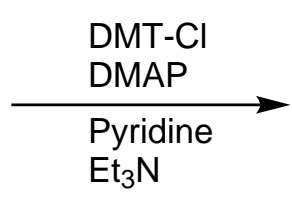

354.10

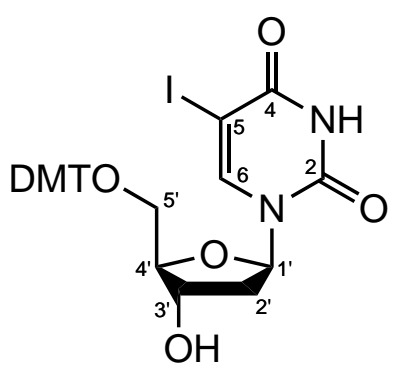

32

656.46

To a solution of 5-iodo-2'-desoxyuridine (1.00 g, $2.82 \mathrm{mmol}$ ) in pyridine (20 mL) 4,4'-dimethoxytrityl chloride (1.91 g, $5.64 \mathrm{mmol}, 2.00$ eq.), 4-(dimethylamino)pyridine (34 mg, $0.28 \mathrm{mmol}, 0.10$ eq.) and triethylamine $(0.59 \mathrm{~mL}, 4.23 \mathrm{mmol}, \rho=0.73 \mathrm{~g} / \mathrm{mL}, 1.50$ eq.) were added. The solution was stirred at ambient temperature for $6 \mathrm{~h}$ under argon atmosphere. The solvent was removed under reduced pressure and the crude product was purified by silica gel flash column chromatography. Elu-

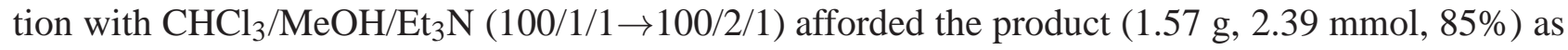
a white foam.

${ }^{1}$ H-NMR (400 MHz, DMSO-d6): $\delta=8.32$ (s, 1H, NH), 8.03 (s, 1H, H6), 7.44-7.20 (m, 9H, DMT), 6.90 (m, 4H, DMT), 6.13 (m, 1H, H1), 4.25 (m, 1H, H3`), 3.92 (m, 1H, H4), 3.75 (s, 6H, $2 \times$ OMe), 3.25-3.16 (m, 2H, H5`a, H5`b), 2.31-2.16 (m, 2H, H2`a, H2`b) ppm.

${ }^{13}$ C-NMR (400 MHz, DMSO-d6): $\delta=161.05$ (1C, C4), 158.57 (2C, DMT), 150.56 (1C, C2), 145.21 (1C, DMT), 144.66 (1C, C6), 135.92 (1C, DMT), 135.85 (1C, DMT), 130,19 (4C, DMT), 128.42 (2C, DMT), 128.13 (2C, DMT), 127.18 (1C, DMT), 113.74 (4C DMT), 86.30 (1C, DMT), 86.27 (1C, C4'), 85.25 (1C, C1́), 71.00 (1C, C3’), 70.31 (1C, C5), 64.18 (1C, C5’), 55.53 (2C, $2 \times \mathrm{OMe}), 40.20$ (1C, C2’) ppm.

ESI-MS $m / z$ (MeCN, negative mode): calc. for $\mathrm{C}_{30} \mathrm{H}_{28} \mathrm{IN}_{2} \mathrm{O}_{7}[\mathrm{M}-\mathrm{H}]^{-}:$655.09; found: 655.21. 


\subsubsection{5'-O-Dimethoxytrityl-5-trimethylsilylethynyl-2'-desoxyuridine}
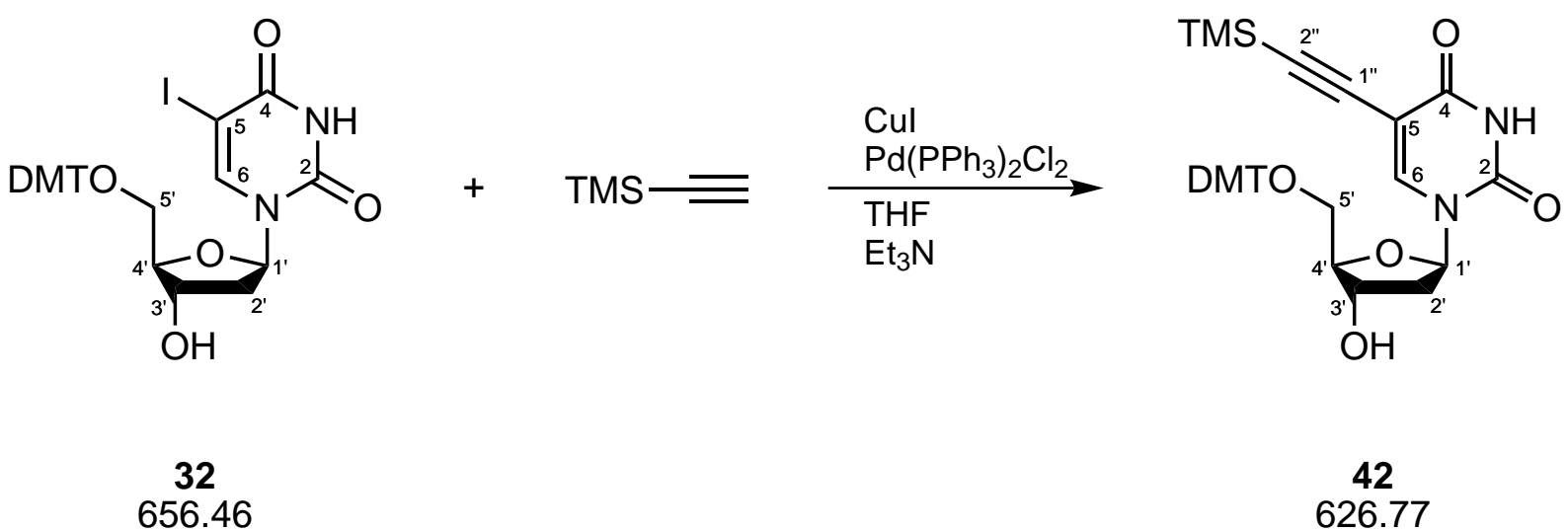

656.46

42
626.77

To a solution of 5'-O-dimethoxytrityl-5-iodo-2'-desoxyuridine (7.75 g, $11.81 \mathrm{mmol})$ in triethylamine $(100 \mathrm{~mL})$ and tetrahydrofuran $(50 \mathrm{~mL})$ were added copper(I) iodide $(179 \mathrm{mg}, 0.94 \mathrm{mmol}$, 0.08 eq.), bis(triphenylphosphine)palladium(II) chloride (246 mg, $0.35 \mathrm{mmol}, 0.03$ eq.) and trimethylsilyl acetylene (3.31 mL, $23.6 \mathrm{mmol}, \rho=0.70 \mathrm{~g} / \mathrm{mL}, 2.00$ eq.). The solution was stirred at $55^{\circ} \mathrm{C}$ for $24 \mathrm{~h}$ under argon atmosphere. The solvent was removed under reduced pressure and the crude product was purified by silica gel flash column chromatography. Elution with $\mathrm{CHCl}_{3} / \mathrm{MeOH} / \mathrm{Et}_{3} \mathrm{~N}$ $(100 / 2 / 1 \rightarrow 100 / 5 / 1)$ afforded the product $(6.89 \mathrm{~g}, 10.99 \mathrm{mmol}, 93 \%)$ as a white foam.

${ }^{1}$ H-NMR (400 MHz, DMSO-d6): $\delta=8.32$ (s, 1H, NH), 7.96 (s, 1H, H6), 7.44-7.18 (m, 9H, DMT), 6.90 (m, 4H, DMT), 6.10 (m, 1H, H1), 4.23 (m, 1H, H3), 3.93 (m, 1H, H4), 3.74 (s, 6H, OMe), 3.17 (m, 2H, H5`a, H5`b), 2.29-2.15 (m, 2H, H2`a, H2`b), 0.02 (s, 9H, TMS) ppm.

${ }^{13}$ C-NMR (400 MHz, DMSO-d6): $\delta=161.90$ (1C, C4), 158.54 (2C, DMT), 149.72 (1C, C2), 145.12 (1C, DMT), 144.07 (1C, C6), 136.08 (1C, DMT), 135.86 (1C, DMT), 130.14 (2C, DMT), 130.06 (2C, DMT), 128.35 (2C, DMT), 128.09 (2C, DMT), 127.10 (1C, DMT), 113.69 (4C DMT), 99.08 (1C, C5), 97.65 (1C, C2”), 97.58 (1C, C1'`), 86.42 (1C, C4`), 86.26 (1C, DMT), 85.56 (1C, C1`), 70.89 (1C, C3), 64.05 (1C, C5’), 55.46 (2C, OMe), 40.33 (1C, C2), 0.02 (3C, TMS) ppm.

ESI-MS $m / z\left(M e C N\right.$, positive mode): calc. for $\mathrm{C}_{41} \mathrm{H}_{54} \mathrm{~N}_{3} \mathrm{O}_{7} \mathrm{Si}\left[\mathrm{M}+\mathrm{Et}_{3} \mathrm{~N}+\mathrm{H}\right]^{+}$: 728.37; found: 728.43 . 


\subsubsection{5'-O-Dimethoxytrityl-5-ethynyl-2'-desoxyuridine}<smiles>CSC#Cc1cn(CC(C)O)c(=O)[nH]c1=O</smiles>

42

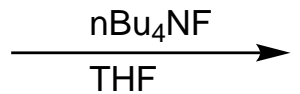

THF

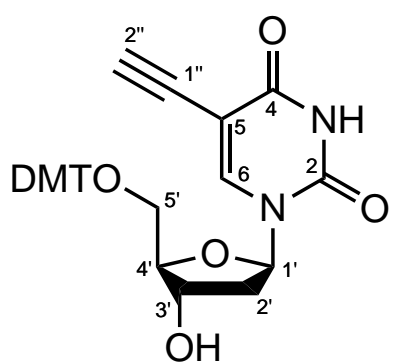

33

554.59

To a solution of 5'-O-dimethoxytrityl-5-trimethylsilylethynyl-2'-desoxyuridine (802 mg, 1.28 $\mathrm{mmol})$ in tetrahydrofuran $(10 \mathrm{~mL})$ tetra- $n$-butylammonium fluoride solution $(1 \mathrm{~mol} / \mathrm{L}$ in THF, 2.56 $\mathrm{mL}, 2.56 \mathrm{mmol}, 2.00$ eq.) was added. The solution was stirred at ambient temperature for $24 \mathrm{~h}$ under argon atmosphere. The solvent was removed under reduced pressure and the crude product was purified by silica gel flash column chromatography. Elution with $\mathrm{CHCl}_{3} / \mathrm{MeOH} / \mathrm{Et}_{3} \mathrm{~N}(100 / 0 / 1 \rightarrow 100 / 1 / 1)$ afforded the product (632 $\mathrm{mg}, 1.14 \mathrm{mmol}, 89 \%)$ as a white foam.

${ }^{1}$ H-NMR (400 MHz, DMSO-d6): $\delta=8.32$ (s, 1H, NH), 7.95 (s, 1H, H6), 7.42-7.19 (m, 9H, DMT), 6.89 (m, 4H, DMT), 6.10 (m, 1H, H1'), 4.24 (m, 1H, H3`), 3.97 (1H, H2”), 3.91 (m, 1H, H4’), 3.74 (s, 6H, OMe), 3.13 (m, 2H, H5`a, H5`b), 2.31-2.15 (m, 2H, H2`a, H2`b) ppm.

${ }^{13}$ C-NMR (400 MHz, DMSO-d6): $\delta=162.12$ (1C, C4), 158.60 (2C, DMT), 149.87 (1C, C2), 145.23 (1C, DMT), 144.36 (1C, C6), 136.01 (1C, DMT), 135.82 (1C, DMT), 130.15 (4C, DMT), 128.34 (2C, DMT), 128.07 (2C, DMT), 127.12 (1C, DMT), 113.69 (4C DMT), 98.36 (1C, C5), 86.33 (1C, C4), 85.54 (1C, C1'), 84.09 (1C, DMT), 76.26 (2C, C1“”, C2’'), 70.87 (1C, C3’), 64.17 (1C, C5’), 55.49 (2C, OMe), 40.05 (1C, C2') ppm.

ESI-MS $m / z\left(\mathrm{MeCN}\right.$, positive mode): calc. for $\mathrm{C}_{38} \mathrm{H}_{46} \mathrm{~N}_{3} \mathrm{O}_{7}\left[\mathrm{M}+\mathrm{Et}_{3} \mathrm{~N}+\mathrm{H}\right]^{+}$: 656.33 ; found: 656.33 . 



\subsubsection{5'-O-Dimethoxytrityl-5-[[(4-trimethylsilylethynyl)phenyl]ethynyl]-2'-desoxyuridine}

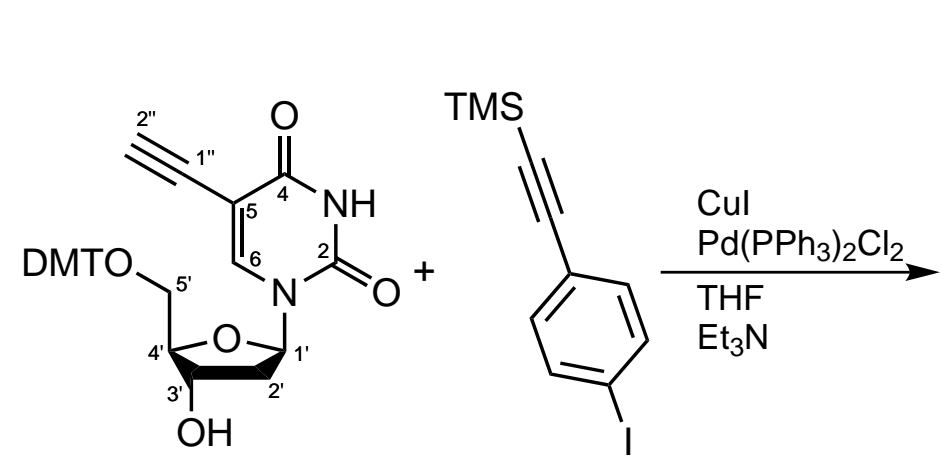

33

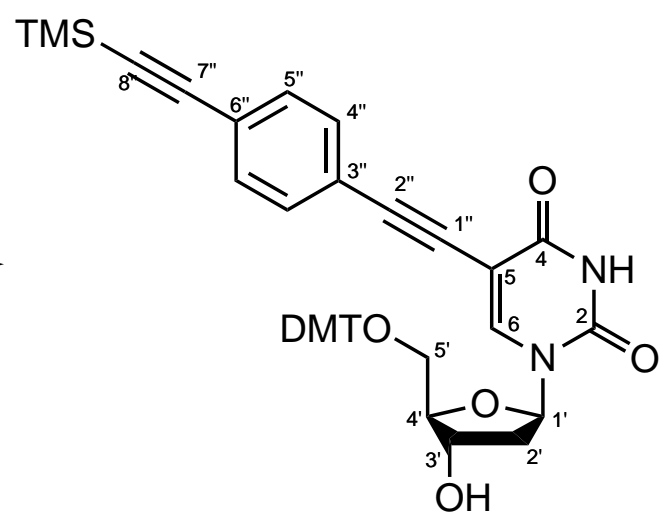

43

726.89

To a solution of 5'-O-dimethoxytrityl-5-ethynyl-2'-desoxyuridine (632 mg, $1.14 \mathrm{mmol}$ ) in triethylamine (30 mL) and tetrahydrofuran (10 mL) were added copper(I) iodide (17 mg, $91 \mu \mathrm{mol}, 0.08$ eq.), bis(triphenylphosphine)palladium(II) cloride ( $24 \mathrm{mg}, 34 \mu \mathrm{mol}, 0.03$ eq.) and (4-iodophenylethynyl)trimethylsilane (684 mg, $2.28 \mathrm{mmol}, 2.00 \mathrm{eq}$.). The solution was stirred at $55^{\circ} \mathrm{C}$ for $24 \mathrm{~h}$ under argon atmosphere. The solvent was removed under reduced pressure and the crude product was purified by silica gel flash column chromatography. Elution with $\mathrm{CHCl}_{3} / \mathrm{MeOH} \mathrm{Et}_{3} \mathrm{~N}(100 / 0 / 1 \rightarrow 100 / 2 / 1)$ afforded the product (670 $\mathrm{mg}, 0.92 \mathrm{mmol}, 81 \%$ ) as a yellow foam.

${ }^{1}$ H-NMR (400 MHz, DMSO-d6): $\delta=8.32$ (s, 1H, NH), 8.12 (s, 1H, H6), 7.42 (m, 2H, DMT), 7.36 (d, $\left.J=8.3 \mathrm{~Hz}, 2 \mathrm{H}, \mathrm{H} 5^{\prime \prime}\right), 7.33-7.14$ (m, $\left.7 \mathrm{H}, \mathrm{DMT}\right), 7.05$ (d, $\left.J=8.3 \mathrm{~Hz}, 2 \mathrm{H}, \mathrm{H} 4^{\prime \prime}\right), 6.85$ (m, 4H, DMT), 6.15 (m, 1H, H1`), 4.33 (m, 1H, H3), 3.97 (m, 1H, H4'), 3.67 (s, 6H, OMe), 3.21 (m, 2H, H5`a, H5`b), 2.36-2.21 (m, 2H, H2`a, H2`b), 0.24 (9H, TMS) ppm.

${ }^{13}$ C-NMR (400 MHz, DMSO-d6): $\delta=161.79$ (1C, C4), 158.54 (2C, DMT), 149.75 (1C, C2), 145.16 (1C, DMT), 143.65 (1C, C6), 136.01 (1C, DMT), 135.84 (1C, DMT), 132.01 (2C, C5”), 131.65 (2C, C4"), 130.09 (4C, DMT), 128.35 (2C, DMT), 128.09 (2C, DMT), 127.20 (1C, DMT), 123.14 (1C, C6"), 122.34 (1C, C9”'), 113.69 (4C DMT), 105.08 (1C, C7”), 98.75 (1C, C5), 96.83 (1C, C8“'), 91.93 (1C, C2”), 86.62 (1C, C4'), 86.40 (1C, DMT), 85.73 (1C, C1'), 84.71 (1C, C1'”), 70.93 (1C, 
C3’), 64.02 (1C, C5'), 55.46 (2C, OMe), 40.46 (1C, C2), 0.25 (3C, TMS) ppm.

ESI-MS $\mathrm{m} / z\left(\mathrm{MeCN}\right.$, positive mode): calc. for $\mathrm{C}_{49} \mathrm{H}_{58} \mathrm{~N}_{3} \mathrm{O}_{7} \mathrm{Si}\left[\mathrm{M}+\mathrm{Et}_{3} \mathrm{~N}+\mathrm{H}\right]^{+}$: 828.40; found: 828.53 . 


\subsubsection{5'-O-Dimethoxytrityl-5-[[(4-ethynyl)phenyl]ethynyl]-2'-desoxyuridine}

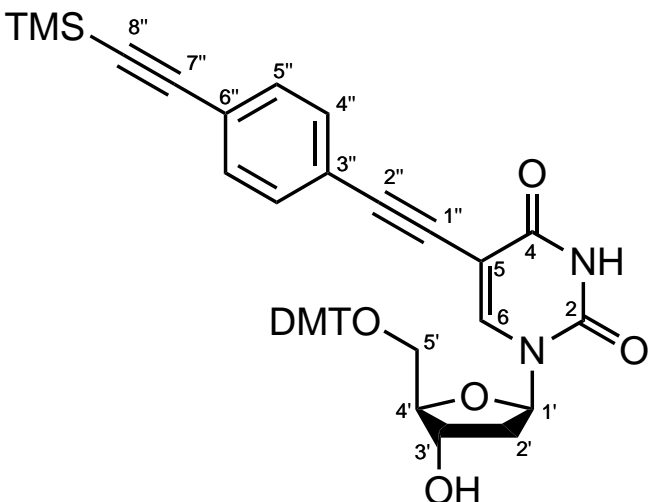

43 726.89

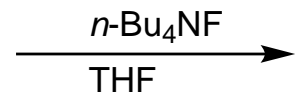

34

654.71

To a solution of 5'-O-dimethoxytrityl-5-[[(4-trimethylsilylethynyl)phenyl]ethynyl]-2'-desoxyuridine $(5.09 \mathrm{~g}, 7.00 \mathrm{mmol})$ in tetrahydrofuran $(200 \mathrm{~mL})$ tetra- $n$-butylammonium fluoride solution ( $1 \mathrm{~mol} / \mathrm{L}$ in THF, $14.0 \mathrm{~mL}, 14.0 \mathrm{mmol}, 2.00$ eq.) was added. The solution was stirred at ambient temperature for $24 \mathrm{~h}$ under argon atmosphere. The solvent was removed under reduced pressure and the crude product was purified by silica gel flash column chromatography. Elution with $\mathrm{CHCl}_{3} / \mathrm{MeOH} / \mathrm{Et}_{3} \mathrm{~N}(100 / 0 / 1 \rightarrow 100 / 5 / 1)$ afforded the product $(4.37 \mathrm{~g}, 6.68 \mathrm{mmol}, 95 \%)$ as a white foam.

${ }^{1}$ H-NMR (400 MHz, DMSO-d6): $\delta=8.33$ (s, 1H, NH), 8.11 (s, 1H, H6), 7.44 (m, 2H, DMT), 7.38 (d, $\left.J=8.3 \mathrm{~Hz}, 2 \mathrm{H}, \mathrm{H}^{\prime \prime}\right), 7.34-7.14$ (m, $\left.7 \mathrm{H}, \mathrm{DMT}\right), 7.07$ (d, $\left.J=8.3 \mathrm{~Hz}, 2 \mathrm{H}, \mathrm{H} 4^{\prime \prime}\right), 6.85$ (m, 4H, DMT), 6.19 (m, 1H, H1`), 4.34 (m, 1H, H3`), 4.32 (1H, H8``), 3.98 (m, 1H, H4), 3.66 (s, 6H, OMe), 3.20 (m, 2H, H5`a, H5`b), 2.29 (m, 2H, H2`a, H2`b) ppm.

${ }^{13}$ C-NMR (400 MHz, DMSO-d6): $\delta=162.45$ (1C, C4), 158.52 (2C, DMT), 150.25 (1C, C2), 145.16 (1C, DMT), 143.53 (1C, C6), 136.01 (1C, DMT), 135.87 (1C, DMT), 132.11 (2C, C5”), 131.61 (2C, C4"'), 130.10 (4C, DMT), 128.32 (2C, DMT), 128.09 (2C, DMT), 127.15 (1C, DMT), 123.30 (1C, C6"'), 121.87 (1C, C9”), 113.68 (4C DMT), 98.75 (1C, C5), 91.60 (1C, C2”) 86.56 (1C, C4’), 86.37

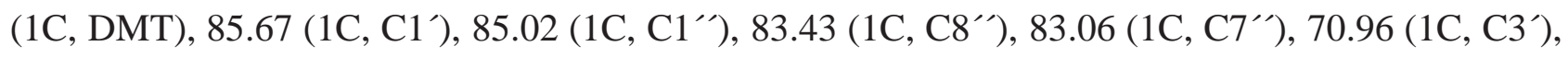


116

64.09 (1C, $\left.\mathrm{C5}^{\prime}\right), 55.45$ (2C, OMe), 40.85 (1C, $\left.\mathrm{C}^{\prime}\right) \mathrm{ppm}$.

ESI-MS $m / z$ (MeCN, positive mode): calc. for $\mathrm{C}_{40} \mathrm{H}_{34} \mathrm{~N}_{3} \mathrm{NaO}_{7}[\mathrm{M}+\mathrm{Na}]^{+}$: 677.23; found: 677.23 . 


\subsubsection{5'-O-Dimethoxytrityl-5-[[(4-ethynyl)phenyl]ethynyl]-2'-desoxyuridine-3'-[O-(2-} cyanoethyl)- $N, N$-diisopropyl]phosphoramidite

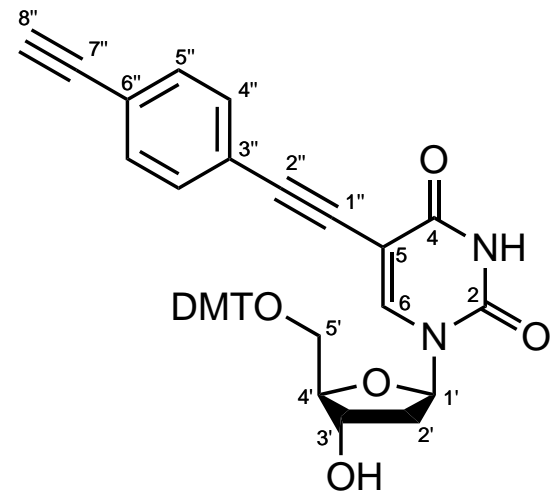

34

654.71
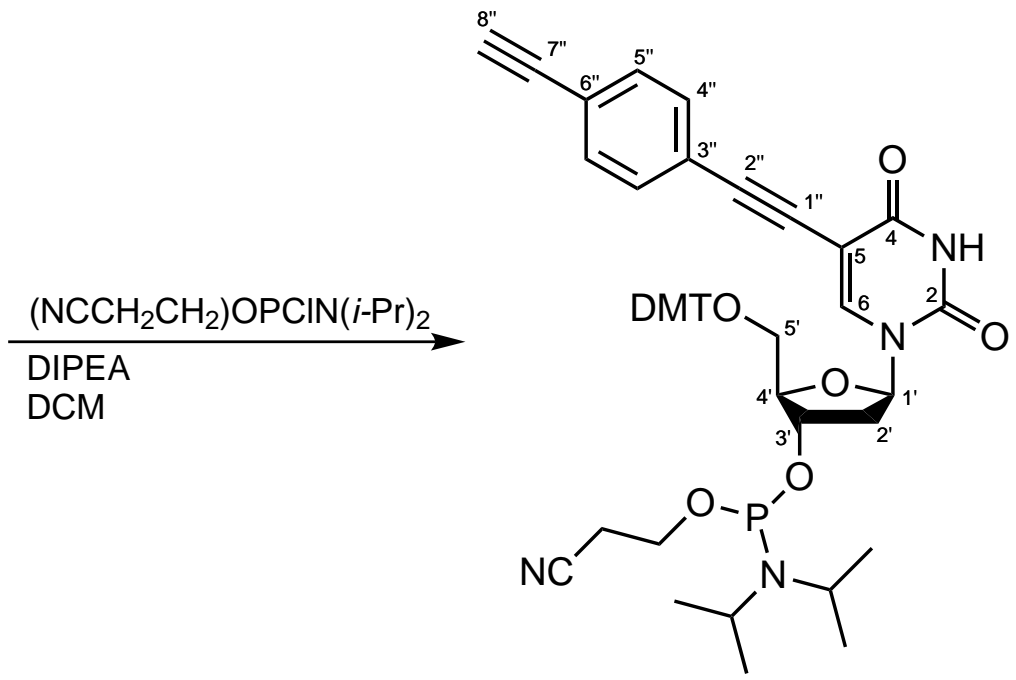

35

To a solution of 5'-O-dimethoxytrityl-5-[[(4-ethynyl)phenyl]ethynyl]-2'-desoxyuridine (537 mg, $0.82 \mathrm{mmol})$ in dichloromethane $(10 \mathrm{~mL})$ were added diisopropylethylamine $(279 \mu \mathrm{L}, 1.64 \mathrm{mmol}$, $\rho=0.76 \mathrm{~g} / \mathrm{mL}, 2.00$ eq.) and 2-cyanoethyl diisopropylchlorophosphoramidite (387 mg, $1.64 \mathrm{mmol}$, 2.00 eq.). The solution was stirred at ambient temperature for $2 \mathrm{~h}$ under argon atmosphere. The solvent was removed under reduced pressure and the crude product was purified by silica gel flash column chromatography. Elution with hexane/ethyl acetate/Et ${ }_{3} \mathrm{~N}(50 / 50 / 1 \rightarrow 0 / 100 / 1)$ afforded the product (637 mg, $0.75 \mathrm{mmol}, 91 \%$ ) as a yellow foam.

${ }^{1}$ H-NMR $\left(400 \mathrm{MHz}, \mathrm{CDCl}_{3}\right): \delta=8.27$ (s, 1H, H6), 7.46 (m, 2H, DMT), 7.36 (d, J = 8.6 Hz, 2H, H5“), 7.30-7.12 (m, 7 H, DMT), 6.89 (d, J = 8.3 Hz, 2H, H4”), 6.79 (m, 4H, DMT), 6.37 (m, 1H, $\mathrm{H}^{\prime}$ ), 4.65 (m, 1H, H3'), 4.21 (m, 1H, H4'), 3.81 (m, 2H, $\left.\mathrm{OC}_{2} \mathrm{CH}_{2} \mathrm{CN}\right), 3.70$ (s, 3H, OMe), 3.69 (s,

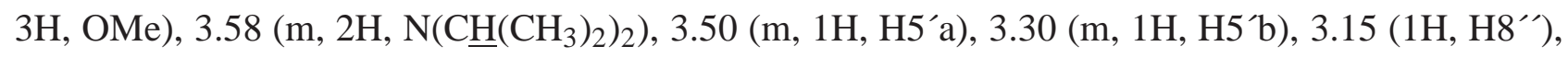

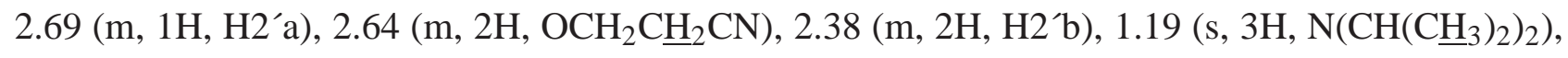

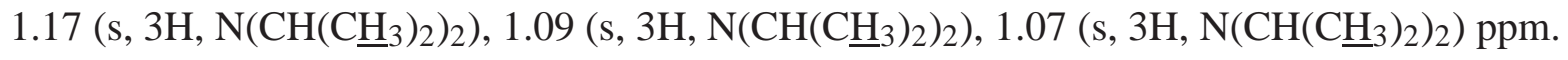


${ }^{13}$ C-NMR (400 MHz, $\mathrm{CDCl}_{3}$ ): $\delta=161.37$ (1C, C4), 158.65 (2C, DMT), 149.33 (1C, C2), 144.36 (1C, DMT), 142.46 (1C, C6), 135.45 (2C, DMT), 131.54 (2C, C5”), 131.43 (2C, C4”), 129.94 (2C, DMT), 129.90 (2C, DMT), 128.02 (2C, DMT), 127.93 (2C, DMT), 127.02 (1C, DMT), 122.97 (1C, C6"), 121.65 (1C, C3"'), 117.56 (1C, $\mathrm{OCH}_{2} \mathrm{CH}_{2} \underline{\mathrm{CN}}$ ), 113.32 (4C DMT), 100.34 (1C, C5), 93.03

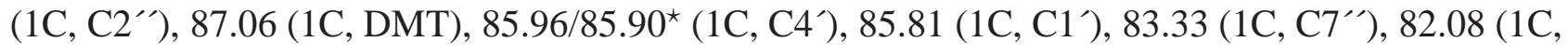
$\left.\mathrm{C}^{\prime \prime}\right), 78.87$ (1C, $\left.\mathrm{C}^{\prime \prime}\right), 73.81 / 73.64^{\star}$ (1C, C3`), 63.18 (1C, C5'), 58.49/58.26* $\left(1 \mathrm{C}, \mathrm{OCH}_{2} \mathrm{CH}_{2} \mathrm{CN}\right)$, $55.14(2 \mathrm{C}, 2 \times \mathrm{OMe}), 43.28\left(1 \mathrm{C}, \mathrm{N}\left(\underline{\mathrm{CH}}\left(\mathrm{CH}_{3}\right)_{2}\right)_{2}\right), 43.16\left(1 \mathrm{C}, \mathrm{N}\left(\underline{\mathrm{CH}}\left(\mathrm{CH}_{3}\right)_{2}\right)_{2}\right), 40.89\left(1 \mathrm{C}, \mathrm{C}^{\prime}\right)$, $24.58\left(1 \mathrm{C}, \mathrm{N}\left(\mathrm{CH}\left(\underline{\mathrm{CH}}_{3}\right)_{2}\right)_{2}\right), 24.51\left(2 \mathrm{C}, \mathrm{N}\left(\mathrm{CH}\left(\underline{\mathrm{CH}}_{3}\right)_{2}\right)_{2}\right), 24.45\left(1 \mathrm{C}, \mathrm{N}\left(\mathrm{CH}\left(\underline{\mathrm{CH}}_{3}\right)_{2}\right)_{2}\right), 20.41 / 20.34^{\star}$ (1C, $\left.\mathrm{OCH}_{2} \underline{\mathrm{CH}}_{2} \mathrm{CN}\right)$ ppm.

Signals marked with * indicate two diastereomers due to the phosphor atom.

ESI-MS $m / z\left(\mathrm{MeCN}\right.$, positive mode): calc. for $\mathrm{C}_{49} \mathrm{H}_{52} \mathrm{~N}_{4} \mathrm{O}_{8} \mathrm{P}[\mathrm{M}+\mathrm{H}]^{+}$: 855.35; found: 855.20 . 


\subsubsection{3-Azidopropan-1-ol}<smiles>OCCCBr</smiles>

44

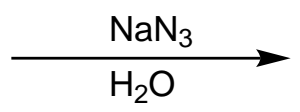

138.99<smiles></smiles>

45

101.11

To a solution of 3-bromopropan-1-ol (12.2 mL, $135 \mathrm{mmol}, \rho=1.54 \mathrm{~g} / \mathrm{mL})$ in water $(100 \mathrm{~mL})$ sodium azide (17.6 g, $270 \mathrm{mmol}, 2.50 \mathrm{eq}$.) was added. The solution was stirred at $80^{\circ} \mathrm{C}$ for $48 \mathrm{~h}$. The mixture was extracted with ethyl acetate $(3 \times 50 \mathrm{~mL})$, the organic layer dried over sodium sulfate, filtrated and the solvent removed under reduced pressure affording the product $(13.44 \mathrm{~g}, 133 \mathrm{mmol}$, 99\%) as a colorless liquid.

${ }^{1} \mathbf{H}-\mathrm{NMR}\left(400 \mathrm{MHz}, \mathrm{CDCl}_{3}\right): \delta=3.60(\mathrm{t}, J=6.0 \mathrm{~Hz}, 2 \mathrm{H}, \mathrm{H1}), 3.32(\mathrm{t}, J=6.6 \mathrm{~Hz}, 2 \mathrm{H}, \mathrm{H} 3), 1.71$ (m, 2H, H2) ppm.

${ }^{13}$ C-NMR (400 MHz, $\left.\mathrm{CDCl}_{3}\right): \delta=59.37(1 \mathrm{C}, \mathrm{C} 1), 48.25$ (1C, C3), 31.38 (1C, C2) ppm.

ESI-MS $m / z$ (MeCN, positive mode): calc. for $\mathrm{C}_{3} \mathrm{H}_{8} \mathrm{~N}_{3} \mathrm{O}[\mathrm{M}+\mathrm{H}]^{+}:$: 102.07; found: 102.00 . 


\subsubsection{3-Azidopropyl acetate}<smiles></smiles>

45

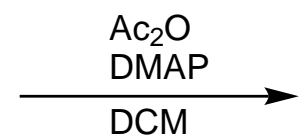

DCM<smiles>CC(=O)OCCCCN</smiles>

46

143.14

To a solution of 3-azidopropan-1-ol (13.4 g, $133 \mathrm{mmol})$ in dichloromethane (100 mL) were added acetic anhydride (24.9 mL, $266 \mathrm{mmol}, \rho=1.09 \mathrm{~g} / \mathrm{mL}, 2.00$ eq.) and 4-(dimethylamino)pyridine (1.59 g, $13 \mathrm{mmol}, 0.10$ eq.). The solution was stirred at ambient temperature for $24 \mathrm{~h}$. The mixture was washed with water $(4 \times 100 \mathrm{~mL})$ and the organic layer was dried over sodium sulfate. After filtration the solvent was removed by evaporation under reduced pressure affording the product (17.4 g, $121 \mathrm{mmol}, 91 \%$ ) as a colorless liquid.

${ }^{1} \mathbf{H}-\mathrm{NMR}\left(400 \mathrm{MHz}_{\mathrm{CDCl}}\right.$ ) : $\delta=4.12(\mathrm{t}, J=6.2 \mathrm{~Hz}, 2 \mathrm{H}, \mathrm{H} 1), 3.36(\mathrm{t}, J=6.6 \mathrm{~Hz}, 2 \mathrm{H}, \mathrm{H} 3), 2.03$ (s, $3 \mathrm{H}, \mathrm{OAc}), 1.88$ (m, 2H, H2) ppm.

${ }^{13}$ C-NMR $\left(400 \mathrm{MHz}, \mathrm{CDCl}_{3}\right): \delta=170.78\left(1 \mathrm{C}, \underline{\mathrm{COCH}}_{3}\right), 61.31$ (1C, C1), 48.18 (1C, C3), 28.10 (1C, C2), $20.83\left(1 \mathrm{C}, \mathrm{COC}_{3}\right) \mathrm{ppm}$.

ESI-MS $m / z\left(M e C N\right.$, positive mode): calc. for $\mathrm{C}_{5} \mathrm{H}_{10} \mathrm{~N}_{3} \mathrm{O}_{2}[\mathrm{M}+\mathrm{H}]^{+}$: 144.08; found: 143.90 . 


\subsubsection{Tris[[1-(3-acetyloxypropyl)-1H-1,2,3-triazol-4-yl]methyl]amine}

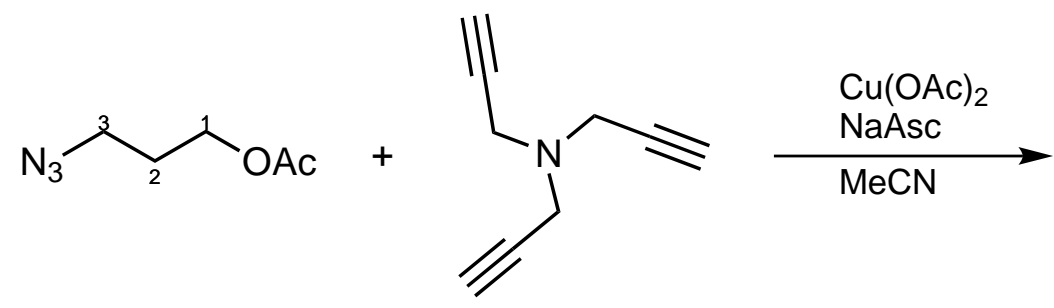

46

143.14
47

560.61

To a solution of tripropargylamine $(4.94 \mathrm{~mL}, 35 \mathrm{mmol}, \rho=0.93 \mathrm{~g} / \mathrm{mL})$ and 3-azidopropyl acetate (17.38 g, $121 \mathrm{mmol}, 3.50$ eq.) in acetonitrile $(25 \mathrm{~mL})$ was added a solution of copper(II) acetate (127 mg, $0.7 \mathrm{mmol}, 0.02$ eq.) and sodium ascorbate (139 mg, $0.7 \mathrm{mmol}, 0.02$ eq.) in water (2 mL). The solution was stirred at $50^{\circ} \mathrm{C}$ for $48 \mathrm{~h}$. The solvents were removed under reduced pressure, the residue was solved in dichloromethane $(50 \mathrm{~mL})$, washed with water $(4 \times 100 \mathrm{~mL})$ and EDTA solution $(0.1 \mathrm{~mol} / \mathrm{L}, 3 \times 50 \mathrm{~mL})$, the organic layer dried over sodium sulfate and filtrated. The solvent was removed under reduced pressure and the crude product was purified by silica gel flash column chromatography. Elution with $\mathrm{CHCl}_{3} / \mathrm{MeOH}(100 / 1 \rightarrow 5)$ afforded the product $(9.24 \mathrm{~g}, 16.5 \mathrm{mmol}$, $47 \%)$ as a yellow oil.

${ }^{1} \mathbf{H}-\mathbf{N M R}\left(400 \mathrm{MHz}, \mathrm{CDCl}_{3}\right.$ ): $\delta=7.77$ (s, 3H, H3), $4.42(\mathrm{t}, J=7.1 \mathrm{~Hz}, 6 \mathrm{H}, \mathrm{H} 4), 4.05$ (t, $J=5.8 \mathrm{~Hz}$, 6H, H6), 3.69 (s, 6H, H1), 2.23 (m, 6H, H5), 2.02 (s, 9H, OAc) ppm.

${ }^{13}$ C-NMR (400 MHz, $\left.\mathrm{CDCl}_{3}\right): \delta=170.81\left(3 \mathrm{C}, \mathrm{COCH}_{3}\right), 143.73$ (3C, C2), 124.16 (3C, C3), 60.90 (3C, C6), 47.09 (3C, C4), 45.91 (3C, C1), 29.37 (3C, C5), 20.82 (3C, $\left.\mathrm{COC} H_{3}\right)$ ppm.

ESI-MS $m / z\left(M e C N\right.$, positive mode): calc. for $\mathrm{C}_{24} \mathrm{H}_{37} \mathrm{~N}_{10} \mathrm{O}_{6}[\mathrm{M}+\mathrm{H}]^{+}:$561.29; found: 561.30 . 


\subsubsection{Tris[[1-(3-hydroxypropyl)-1H-1,2,3-triazol-4-yl]methyl]amine}

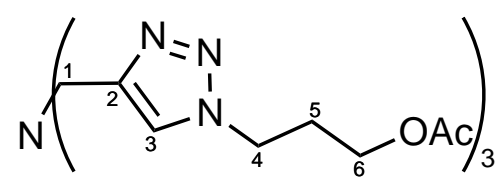

47

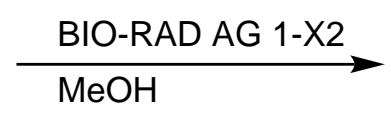

560.61

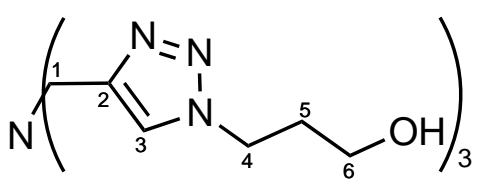

30

434.50

To a solution of tris[[1-(3-acetyloxypropyl)-1H-1,2,3-triazol-4-yl]methyl]amine (9.24 g, 16.5 $\mathrm{mmol})$ in methanol (100 mL) BIO-RAD Strong Anion Exchange AG 1-X2 was added (20 g). The solution was shaken at ambient temperature for $1 \mathrm{~h}$. After filtration the solvent was removed under reduced pressure affording the product $(6.09 \mathrm{~g}, 14.0 \mathrm{mmol}, 85 \%)$ as a white solid.

${ }^{1}$ H-NMR (400 MHz, DMSO-d6): $\delta=8.00$ (s, 3H, H3), 4.38 (t, $\left.J=7.2 \mathrm{~Hz}, 6 \mathrm{H}, \mathrm{H} 4\right), 3.59$ (s, 6H, H1), 3.36 (m, 6H, H6), 1.93 (m, 6H, H5) ppm.

${ }^{13}$ C-NMR (400 MHz, DMSO-d6): $\delta=143.84$ (3C, C2), 124.42 (3C, C3), 57.93 (3C, C6), 47.52 (3C, C1), 47.00 (3C, C4), 33.41 (3C, C5) ppm.

ESI-MS $m / z$ (MeCN, positive mode): calc. for $\mathrm{C}_{18} \mathrm{H}_{31} \mathrm{~N}_{10} \mathrm{O}_{3}[\mathrm{M}+\mathrm{H}]^{+}:$435.26; found: 435.20 . 


\subsubsection{4-Azido- $\alpha, \alpha, \alpha$-tris[[N,N-di(carboxymethyl)amino]methyl]toluene}

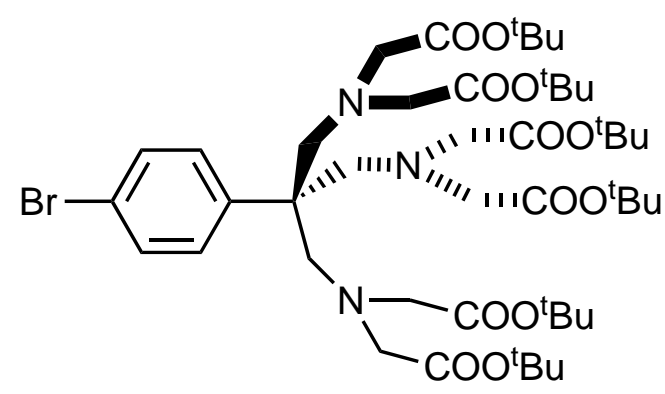

40b

943.01

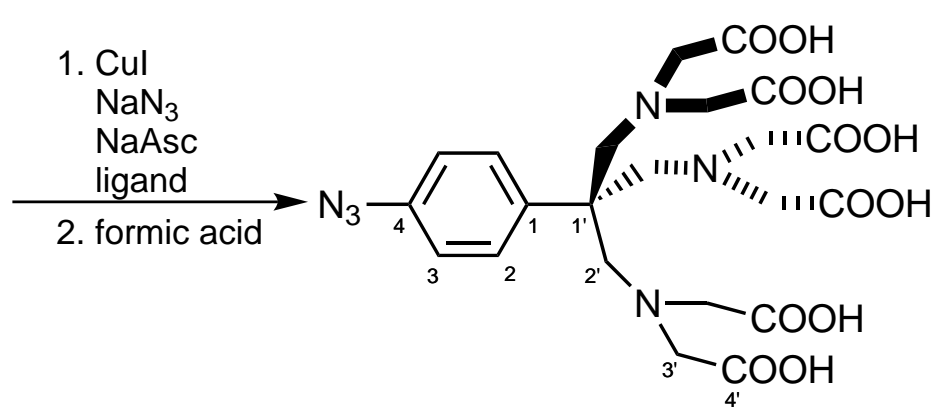

36

568.49

To a solution of 4-Bromo- $\alpha, \alpha, \alpha$-tris[[ $N, N$-di(tert-butoxycarbonylmethyl)amino $]$ methyl $]$ toluene (909 mg, $964 \mu \mathrm{mol}$ ) in EtOH/DMSO/ $\mathrm{H}_{2} \mathrm{O}(18 \mathrm{~mL}, 9 / 6 / 3)$ were added copper(I) iodide (36.7 mg, $193 \mu \mathrm{mol}, 0.2$ eq.), sodium azide (313 mg, $4.82 \mathrm{mmol}, 5$ eq.), sodium ascorbate (19.1 mg, 96.4 $\mu$ mol, 0.1 eq.) and trans- $N, N^{\prime}$-dimethylcyclohexane-1,2-diamine. The reaction mixture was stirred at $100^{\circ} \mathrm{C}$ for $72 \mathrm{~h}$. The solvents were removed under reduced pressure, the residue was dissolved in ethyl acetate $(40 \mathrm{~mL})$, washed with water $(4 \times 40 \mathrm{~mL})$ and the organic layer dried over sodium sulfate. After filtration the solvent was removed under reduced pressure. The residue was dissolved in formic acid $(10 \mathrm{~mL})$ and stirred at ambient temperature for $120 \mathrm{~h}$. After addition of water $(5 \mathrm{~mL})$ the solvents were removed under reduced pressure. The crude product was purified by HPLC. The gradient used is shown below. Combined product fractions (retention time $=15.62 \mathrm{~min}$ ) afforded the product after lyophilization as a white solid (112 mg, $197 \mu \mathrm{mol}, 20 \%)$.

${ }^{1} \mathbf{H}-\mathbf{N M R}\left(400 \mathrm{MHz}, \mathrm{D}_{2} \mathrm{O}\right): \delta=7.52(\mathrm{~d}, J=8.6 \mathrm{~Hz}, 2 \mathrm{H}, \mathrm{H} 2), 7.10(\mathrm{~d}, J=8.6 \mathrm{~Hz}, 2 \mathrm{H}, \mathrm{H} 3), 3.62(\mathrm{~s}$, 6H, H2'), 3.60 (s, 12H, H3') ppm.

${ }^{13}$ C-NMR (400 MHz, $\mathrm{D}_{2} \mathrm{O}$ ): $\delta=172.68$ (6C, C4'), 139.88 (1C, C4), 134.81 (1C, C1), 128.13 (2C, C3), 119.79 (2C, C2), 61.02 (3C, C2'), 56.10 (6C, C3), 45.53 (1C, C1') ppm.

ESI-MS $m / z$ (MeCN, positive mode): calc. for $\mathrm{C}_{22} \mathrm{H}_{29} \mathrm{~N}_{6} \mathrm{O}_{12}[\mathrm{M}+\mathrm{H}]^{+}:$569.18; found: 569.26. 


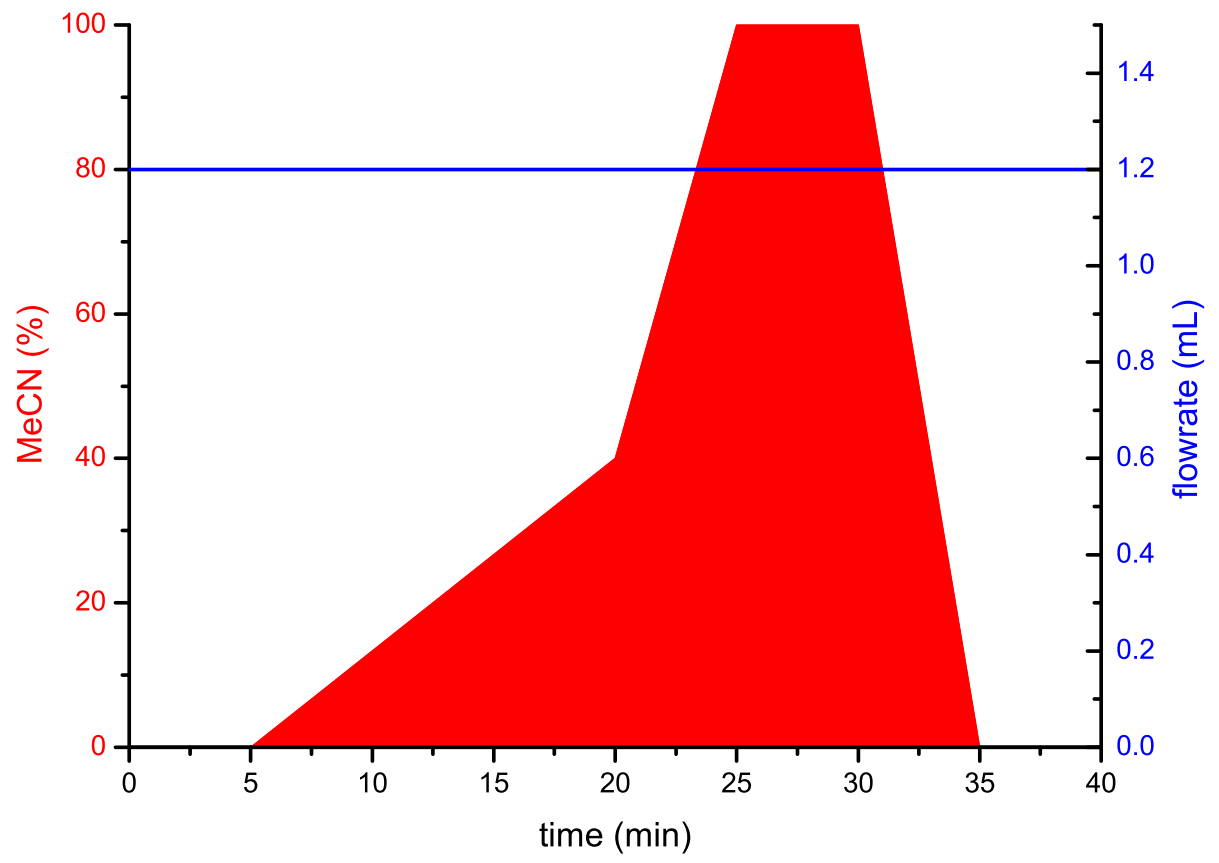




\subsubsection{Click Reaction On Modified DNA In Solution}

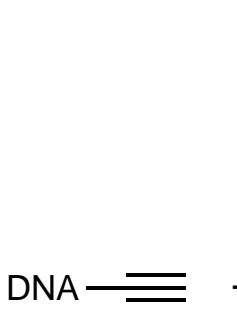

37

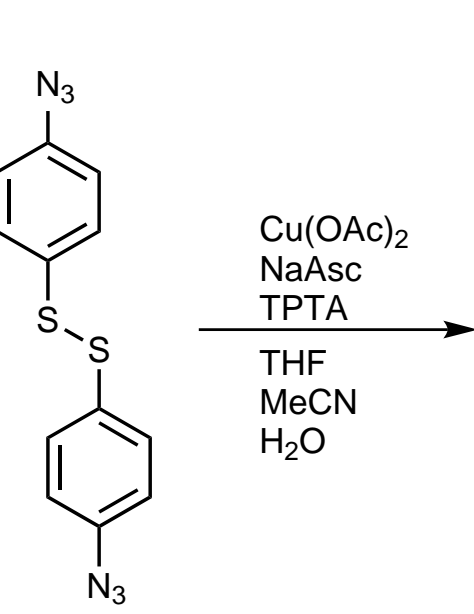

38

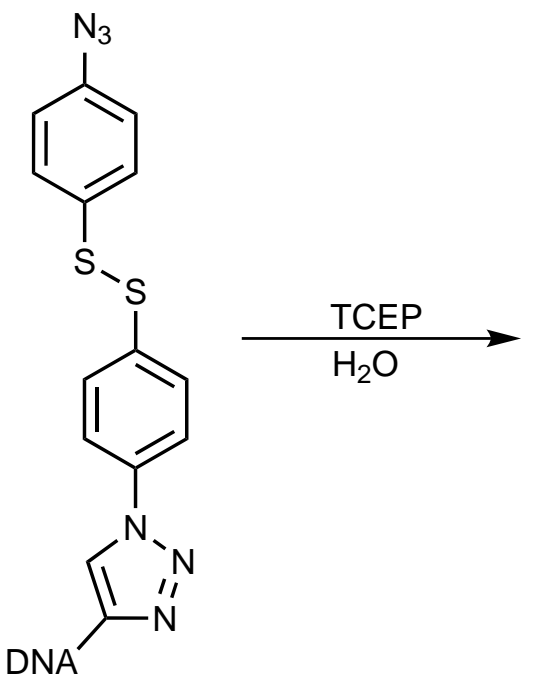

48

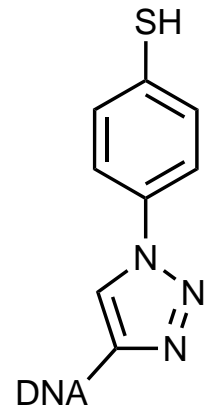

39

To a solution of modified DNA $(2.8 \mu \mathrm{mol})$ in sodium chloride solution $(1 \mathrm{~mol} / \mathrm{L}, 4 \mathrm{~mL})$ were added a freshly prepared solution of copper(II) acetate (14.0 mg, $70 \mu \mathrm{mol}, 25$ eq.), sodium ascorbate (13.9 mg, $70 \mu \mathrm{mol}, 25$ eq.) and tris[[1-(3-hydroxypropyl)-1H-1,2,3-triazol-4-yl]methyl]amine (30.4 mg, $70 \mu \mathrm{mol}, 25$ eq.) in water $(2.0 \mathrm{~mL})$ and a solution of 4,4'-dithiobis(phenylazide) (42.1 mg, $140 \mu \mathrm{mol}, 50$ eq.) in $\mathrm{H}_{2} \mathrm{O} / \mathrm{MeCN} / \mathrm{THF}$ ( $\left.3 \mathrm{~mL}, 1 / 1 / 2\right)$. The reaction mixture was stirred at $55^{\circ} \mathrm{C}$ for $3 \mathrm{~h}$. THF and MeCN were removed under reduced pressure. After centrifugation the residue was washed with water $(1 \mathrm{~mL})$ and the combined supernatants were reduced to $500 \mu \mathrm{L}$ with a Sartorius Vivaspin $2 \mathrm{~mL}$ concentration device with a MWCO of $2000 \mathrm{~g} / \mathrm{mol}$ and washed with EDTA solution $(5 \%, \mathrm{pH} 7,3 \times 5 \mathrm{~mL})$. To the intermediate product was added tris(2-carboxyethyl)phosphine hydrochloride (TCEP) $(40.1 \mathrm{mg}, 140 \mu \mathrm{mol}, 50$ eq.) and the solution was shaken at ambient temperature for $12 \mathrm{~h}$. HPLC purification afforded one DNA peak with a retention time of $22.87 \mathrm{~min}$. The gradient used is shown at the tagging protocol. Mass spectrometry showed only free thiol DNA $(\mathrm{M}=7564.78 \mathrm{~g} / \mathrm{mol})$. UV-VIS concentration determination: $1.75 \mu \mathrm{mol}, 63 \%$. The product was subsequently lyophilized and stored at $-25^{\circ} \mathrm{C}$. 


\subsection{Tagging Protocol}

To a solution of Cys-Ph-TAHA tag $(1.02 \mathrm{~mL}, 7.5$ eq., $13.1 \mu \mathrm{mol}, 13.3 \mathrm{~mol} / \mathrm{L})$ lathanide trichloride solution $(0.30 \mathrm{~mL}, 9.0$ eq., $15.8 \mu \mathrm{mol}, 53.6 \mathrm{mmol} / \mathrm{L})$ was added. The solution was shaken at ambient temperature for $2 \mathrm{~h}$. The $\mathrm{pH}$ was adjusted to 7.1 with $0.1 \mathrm{~mol} / \mathrm{L}$ sodium hydroxide solution. After centrifugation the supernatant was added to the lyophilized free thiol DNA $(1.75 \mu \mathrm{mol})$ and shaken at ambient temperature for $12 \mathrm{~h}$. HPLC purification afforded one DNA peak with a retention time of 23.38 min. The gradient used is shown below. Mass spectrometry showed only tagged DNA. The solution containing the product was reduced to $500 \mu \mathrm{L}$ with a Millipore Amicon $15 \mathrm{~mL}$ concentration device with a MWCO of $3000 \mathrm{~g} / \mathrm{mol}$, washed with sodium chloride solution $(1 \mathrm{~mol} / \mathrm{L}$, pH 7.6, $50 \mathrm{mmol} / \mathrm{L}$ MOPS-buffer, $10 \times 15 \mathrm{~mL})$, reduced to $100 \mu \mathrm{L}$, diluted with $150 \mu \mathrm{L} \mathrm{D} \mathrm{D}_{2} \mathrm{O}(0.1 \mathrm{~mol} / \mathrm{L}$ $\mathrm{NaCl}, \mathrm{pH} 7$, deuterium corrected) and transferred to a Shigemi NMR tube.

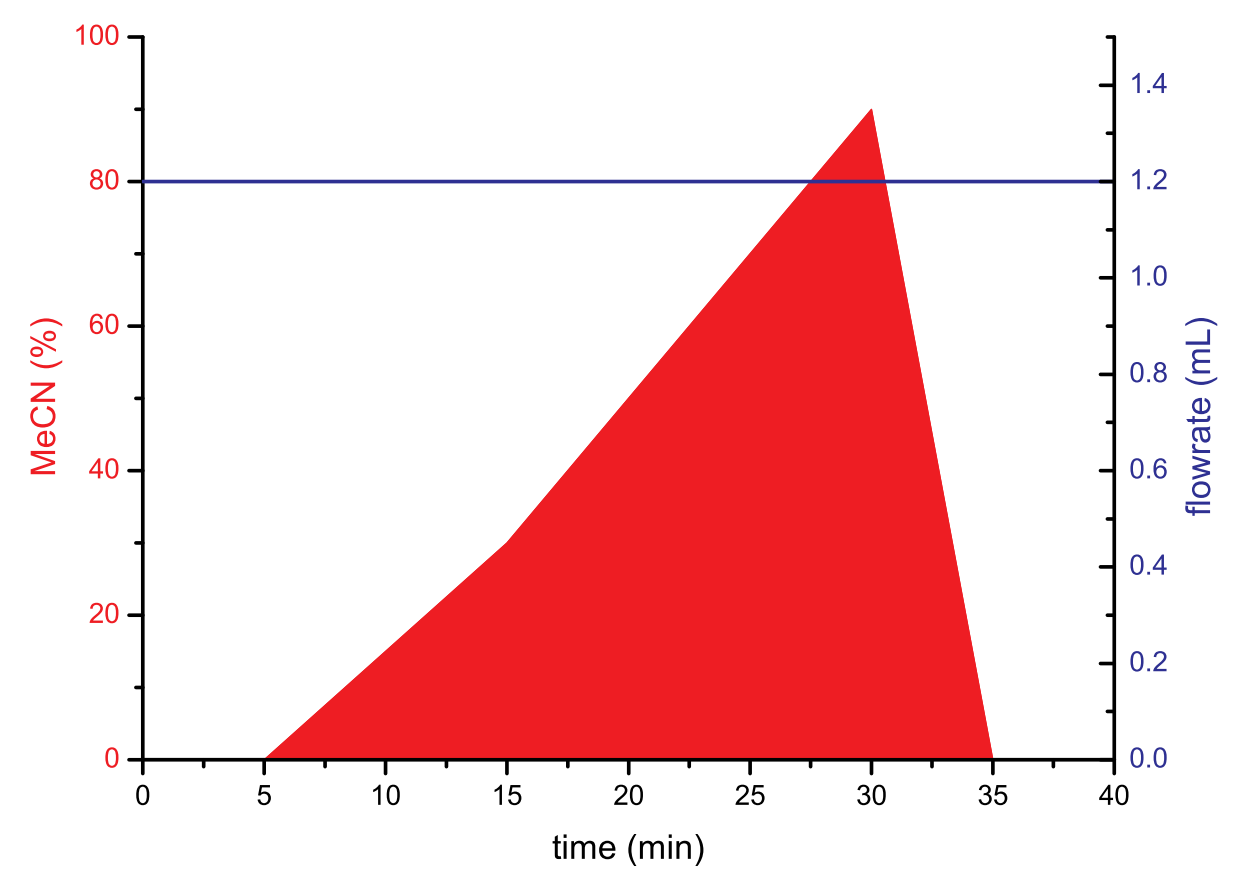




\section{References}

[Abragam1961] A. Abragam, Principles of Nuclear Magnetism, Oxford Science Publications, New York, 1961.

[Adrian1970] F. J. Adrian, J. Chem. Phys. 1970, 53, 3374-3375.

[Adrian1977a] $\quad$ F. J. Adrian, Radical Pair Mechanism Of Chemically Induced Magnetic Polarization. In Chemically Induced Magnetic Polarization, 34; L. T. Muus, P. W. Atkins, K. A. McLauchlan, J. B. Pedersen, Eds., D. Reidel, Dordrecht, 1977.

[Adrian1977b] F. J. Adrian, Triplet Overhauser Mechanism of CIDNP. In Chemically Induced Magnetic Polarization, 34; L. T. Muus, P. W. Atkins, K. A. McLauchlan, J. B. Pedersen, Eds., D. Reidel, Dordrecht, 1977.

[Al-Hashimi2001a] H. M. Al-Hashimi, A. Majumdar, A. Gorin, A. Kettani, E. Skripkin, D. J. Patel, J. Am. Chem. Soc. 2001, 123, 633-640.

[Al-Hashimi2001b] H. M. Al-Hashimi, J. R. Tolman, A. Majumdar, A. Gorin, D. J. Patel, J. Am. Chem. Soc. 2001, 123, 5806-5807.

[Anderson2005] J. Anderson, U. Madsen, F. Bjoerkling, X. Liang, Synlett 2005, 14, 22092213.

[Atkins1977] P. W. Atkins, The Triplet Mechanism. In Chemically Induced Magnetic Polarization, 34; L. T. Muus, P. W. Atkins, K. A. McLauchlan, J. B. Pedersen, Eds., D. Reidel, Dordrecht, 1977.

[Bargon1967] J. Bargon, H. Fischer, U. Johnsen, Z. Naturforsch. 1967, 22a, 1551-1555.

[Bargon1979] J. Bargon, G. P. Gardini, J. Am. Chem. Soc. 1979, 101, 7732-7733.

[Bax1997] A. Bax, N. Tjandra, J. Biomol. NMR 1997, 10, 289-292.

[Bennett1978] L. H. Bennett, C. H. Page, L. J. Swartzendruber, J. Res. Natl. Bur. Stand. (U. S.) 1978, 83, 9-12.

[Bertini2002] I. Bertini, C. Luchinat, G. Parigi, Prog. Nucl. Mag. Res. Sp. 2002, 40, 249273. 
[Bertini2003]

[Bertini2004]

[Bloch1946a]

[Bloch1946b]

[Bloembergen1948]

[Bloembergen1957a]

[Bothner-By1981]

[Bothner-By1996]

[Bronstein2008]

[Bryce2004]

[Burrows1998]

[Caruthers1991]

[Cavanagh2007]

[Chan2004]

[Clore2009]

[Closs1969a]

[Closs1969b]
I. Bertini, I. Gelis, N. Katsaros, C. Luchinat, A. Provenzani, Biochemistry 2003, 42, 8011-8021.

I. Bertini, C. Del Bianco, I. Gelis, N. Katsaros, C. Luchinat, G. Parigi, M. Peana, A. Provenzani, M. A. Zoroddu, Proc. Natl. Acad. Sci. U.S.A. 2004, $101,6481-6846$.

F. Bloch, W. W. Hansen, M. Packard, Phys. Rev. 1946, 69, 3.

F. Bloch, Phys. Rev. 1946, 70, 460-474.

N. Bloembergen, E. M. Purcell, R. V. Pound, Phys. Rev. 1948, 73, 679-712.

N. Bloembergen, J. Chem. Phys. 1957, 27, 572-573.

A. A. Bothner-By, J. Domaille, C. Gayathri, J. Am. Chem. Soc. 1981, 103, 5602-5603.

A. A. Bothner-By, Magnetic Field Induced Alignment of Molecules. In Encyclopedia of Nuclear Magnetic Resonance, 5; D. M. Grant, R. K. Harris, Eds., Wiley, Chichester, 1996.

I. N. Bronstein, K. A. Semendjajew, G. Musiol, H. Mühlig, Taschenbuch der Mathematik, Harri, Frankfurt am Main, 7. Auflage, 2008.

D. L. Bryce, J. Boisbouvier, A. Bax, J. Am. Chem. Soc. 2004, 126, 1082010821.

C. J. Burrows, J. G. Muller, Chem. Rev. 1998, 98, 1109-1151.

M. H. Caruthers, Acc. Chem. Res. 1991, 24, 278-284.

J. Cavanagh, W. J. Fairbrother, A. G. Palmer III, M. Rance, N. J. Skelton, Protein NMR Spectroscopy, Elsevier, New York, Second Edition, 2007.

T. R. Chan, R. Hilgrad, K. Barry Sharpless, V. V. Fokin, Org. Lett. 2004, 6, 2853-2855.

G. M. Clore, J. Iwahara, Chem. Rev. 2009, 109, 4108-4139.

G. L. Closs, J. Am. Chem. Soc. 1969, 91, 4552-4554.

G. L. Closs, L. E. Closs, J. Am. Chem. Soc. 1969, 91, 4549-4550. 
[Closs1970]

[CRC2002]

[Dasgupta2011]

[Dorai2001]

[Dvoretsky2002]

[Feynman1964]

[Forbes2012]

[Franklin1994]

[Gaponenko2000]

[Gaponenko2002]

[Gayathri1982]

[Gerthsen2006]

[Gierlich2006]

[Goez1995]

[Goez2005]

[Goldman1984]

[Graham2011]
G. L. Closs, A. D. Trifunac, J. Am. Chem. Soc. 1970, 92, 2183-2184. Handbook of Chemistry and Physics, CRC Press, Boca Raton, 83, 2002. S. Dasgupta, X. Hu, P. H. J. Keizers, W. M. Liu, C. Luchinat, M. Nagulapalli, M. Overhand, G. Parigi, L. Sgheri, M. Ubbink, J. Biomol. NMR 2011, $51,253-263$.

K. Dorai, A. Kumar, Chem. Phys. Lett. 2001, 335, 176-182.

A. Dvoretsky, V. Gaponenko, P. R. Rosevear, FEBS Lett. 2002, 528, 189192.

R. P. Feynman, L. R. B., M. Sands, The Feynman Lectures on Physics Book 2 Electromagnetism and Matter, Addison-Wesley, Oxnard, 1964.

C. R. Forbes, N. J. Zondlo, Org. Lett. 2012, 14, 464-467.

S. J. Franklin, K. N. Raymond, Inorg. Chem. 1994, 33, 5794-5804.

V. Gaponenko, A. Dvoretsky, C. Walsby, B. M. Hoffman, P. R. Rosevear, Biochemistry 2000, 39, 15217-15224.

V. Gaponenko, A. S. Altieri, J. Li, A. Byrd, J. Biomol. NMR 2002, 24, 143148.

C. Gayathri, A. A. Bothner-By, P. C. M. van Zijl, C. Maclean, Chem. Phys. Lett. 1982, 87, 192-196.

C. Gerthsen, Gerthsen Physik, Springer, Berlin, 23., überarbeite Auflage, 2006.

J. Gierlich, G. A. Burley, P. M. E. Gramlich, D. M. Hammond, T. Carell, Org. Lett. 2006, 8, 3639-3642.

M. Goez, Concept. Magnetic. Res. 1995, 7, 69-86.

M. Goez, I. Kuprov, P. J. Hore, J. Magn. Reson. 2005, 177, 139-145.

M. Goldman, J. Magn. Reson. 1984, 60, 437-452.

B. Graham, C. T. Loh, J. D. Swarbrick, P. Ung, J. Shin, H. Yagi, X. Jia, S. Chhabra, N. Barlow, G. Pintacuda, T. Huber, G. Otting, Bioconjugate Chem. 2011, 22, 2118-2125. 
[Gueron1975]

[Haberz2005]

[Haberz2006]

[Haeussinger2009]

[Hansen1998]

[Harms 1997]

[Hausser1968]

[Heelis1982]

[Heelis1991]

[Hein2011]

[Himo2005]

[Hore1979b]

[Hore1993]

[Hubbard1958]

[Hubbard1961]

[Huisgen1963]

[Ikegami2004]
M. Gueron, J. Magn. Reson. 1975, 19, 58-66.

P. Haberz, J. Farjon, C. Griesinger, Angew. Chem., Int. Ed. 2005, 117, 431433.

P. Haberz, F. Rodriguez-Castaneda, J. Junker, S. Becker, A. Leonov, C. Griesinger, Org. Lett. 2006, 8, 1275-1278.

D. Haeussinger, J. Huang, S. Grzesiek, J. Am. Chem. Soc. 2009, 131, 14761-14767.

M. R. Hansen, L. Mueller, A. Pardi, Nat. Struct. Biol. 1998, 5, 1065-1074.

G. S. Harms, S. W. Pauls, J. F. Hedstrom, C. K. Johnson, J. Fluoresc. 1997, 7, 273-282.

K. H. Hausser, D. Stehlik, Adv. Magn. Reson. 1968, 3, 79-139.

P. F. Heelis, Chem. Soc. Rev. 1982, 11, 15-39.

P. F. Heelis, The Photochemistry of Flavins. In Chemistry and Biochemistry of Flavoenzyms, 1; F. Müller, Eds., CRC Press, Boca Raton, 1991.

J. E. Hein, L. B. Krasnova, M. Iwasaki, V. V. Fokin, Org. Synth. 2011, 88, $238-246$.

F. Himo, T. Lovell, R. Hilgraf, V. Rostovtsev, L. Noodleman, K. B. Sharpless, V. V. Fokin, J. Am. Chem. Soc. 2005, 127, 210-216.

P. J. Hore, Chemically Induced Dynamic Electron Polarization. In Specialist Periodical Reports, 5; P. B. Ayscough, Eds., The chemical Society, London, 1979.

P. J. Hore, R. W. Broadhurst, Prog. Nucl. Mag. Res. Sp. 1993, 25, 345-402.

P. S. Hubbard, Phys. Rev. 1958, 109, 1153-1158.

P. S. Hubbard, Rev. Mod. Phys. 1961, 33, 249-264.

R. Huisgen, Angew. Chem. 1963, 75, 604-637.

T. Ikegami, L. Verdier, P. Sakhaii, S. Grimme, B. Pescatore, K. Saxena, K. M. Fiebig, C. Griesinger, J. Biomol. NMR 2004, 29, 339-349. 
[Ivanov2011] K. L. Ivanov, N. N. Lukzen, O. B. Morozova, K. H. Mok, A. V. Yurkovskaya, Appl. Magn. Reson. 2011, 41, 251-266.

[Jia2011a] X. Jia, A. Maleckis, T. Huber, G. Otting, Chem. Eur. J. 2011, 17, 68306836.

[Jia2011b]

X. Jia, H. Yagi, X. C. Su, M. Stanton-Cook, T. Huber, G. Otting, J. Biomol. NMR 2011, 50, 411-420.

[John2007]

M. John, G. Otting, ChemPhysChem 2007, 8, 2309-2313.

[Kaptein1969]

R. Kaptein, J. L. Oosterhoff, Chemical Physics Letters 1969, 4, 195-197.

[Kaptein1971]

R. Kaptein, J. Chem. Soc. D 1971, 14, 732-733.

[Kaptein1972]

R. Kaptein, J. Am. Chem. Soc. 1972, 94, 6251-6262.

[Kaptein1978]

[Keeler2010]

[Keizers2007]

R. Kaptein, K. Dijkstra, F. Muller, J. Magn. Reson. 1978, 31, 171-176.

J. Keeler, Understanding NMR Spectroscopy, Wiley, Chichester, Second Edition, 2010.

P. H. J. Keizers, J. F. Desreux, M. Overhand, M. Ubbink, J. Am. Chem. Soc. 2007, 129, 9292-9293.

[Keizers2008]

P. H. J. Keizers, A. Saragliadis, Y. Hiruma, M. Overhand, M. Ubbink, J. Am. Chem. Soc. 2008, 130, 14802-14812.

[Kiryutin2007] A. S. Kiryutin, O. B. Morozova, L. T. Kuhn, A. V. Yurkovskaya, P. J. Hore, J. Phys. Chem. B 2007, 111, 11221-11227.

[Kobzar2005] K. Kobzar, H. Kessler, B. Luy, Angew. Chem., Int. Ed. 2005, 44, 31453147.

[Kramer2004] F. Kramer, H. Deshmukh, H. Kessler, S. J. Glaser, Concepts Magn. Reson., Part A 2004, 21A, 10-21.

[Kumar2000] A. Kumar, R. Christy Rani Grace, P. K. Madhu, Prog. Nucl. Mag. Res. Sp. 2000, 37, 191-319.

[Kummerloewe2007] G. Kummerloewe, J. Auernheimer, A. Lendlein, B. Luy, J. Am. Chem. Soc. 2007, 129, 6080-6081. 
[Kung1995]

[Kuprov2004a]

[Kuprov2004b]

[Kuprov2005]

[Kuprov2005a]

[Kuprov2007]

[Lakowicz1983]

[Lee2013]

[Leonov2005]

[Levitt2001]

[Li2012]

[Lingwood2011]

[Liu2012]

[Loh2013]

[Lyon1999]
H. C. Kung, K. Y. Wang, I. Goljer, P. H. Bolton, J. Magn. Reson. 1995, 109, $323-325$.

I. Kuprov, P. J. Hore, J. Magn. Reson. 2004, 168, 1-7.

I. Kuprov, P. J. Hore, J. Magn. Reson. 2004, 171, 171-175.

I. Kuprov, M. Goez, P. A. Abbott, P. J. Hore, Rev. Sci. Instrum. 2005, 76, 084103-1-084103-8.

I. Kuprov, Chemically Induced Dynamic Nuclear Polarization of $19 \mathrm{~F} \mathrm{Nu}$ clei, $\mathrm{PhD}$ thesis, Oxford, 2005.

I. Kuprov, T. D. Craggs, S. E. Jackson, P. J. Hore, J. Am. Chem. Soc. 2007, 129, 9004-9013.

J. R. Lakowicz, B. P. Maliwal, J. Biol. Chem. 1983, 258, 4974-4801.

J. H. Lee, S. Cavagnero, J. Phys. Chem. 2013, 117, 6069-6081.

A. Leonov, B. Voigt, F. Rodriguez-Castaneda, P. Sakhaii, C. Griesinger, Chem. Eur. J. 2005, 11, 3342-3348.

M. H. Levitt, Spin dynamics, Wiley, Chichester, 2001.

Q. F. Li, Y. Yang, A. Maleckis, G. Otting, X. C. Su, Chem. Commun. (Cambridge, U. K.) 2012, 48, 2704-2706.

M. D. Lingwood, S. Han, Solution-State Dynamic Nuclear Polarization. In Annual Reports on NMR Spectroscopy, 73; A. G. Webb, Eds., Elsevier, Amsterdam, 2011.

W. M. Liu, P. H. J. Keizers, M. A. S. Hass, A. Blok, M. Timmer, A. C. J. Sarris, M. Overhand, M. Ubbink, J. Am. Chem. Soc. 2012, 134, 1730617313.

C. T. Loh, K. Ozawa, K. L. Tuck, N. Barlow, T. Huber, G. Otting, B. Graham, Bioconjugate Chem. 2013, 24, 260-268.

C. E. Lyon, J. A. Jones, C. Redfield, C. M. Dobson, P. J. Hore, J. Am. Chem. Soc. 1999, 131, 6505-6506. 
[Man2010]

[Matteucci1981]

[McConnell1958]

[Monchick1978]

[Morozova2004]

[Neumann1848]

[Nicholas2010]

[Nordlund1986]

[Noyes 1954]

[Ottiger1998a]

[Ottiger1998b]

[Otting2008]

[Otting2010]

[Pedersen1973a]

[Pedersen1973b]

[Pedersen1974]

[Pedersen1975]

[Peters2011]

[Porcal2003]
B. Man, X. C. Su, H. Liang, S. Simonson, T. Huber, B. A. Messerle, G. Otting, Chem. Eur. J. 2010, 16, 3827-3832.

M. D. Matteucci, M. H. Caruthers, J. Am. Chem. Soc. 1981, 103, 31853191.

H. M. McConnell, R. E. Robertson, J. Chem. Phys. 1958, 29, 1361-1365.

L. Monchick, F. J. Adrian, J. Chem. Phys. 1978, 68, 4376-4383.

O. B. Morozova, A. V. Yurkovskaya, R. Z. Sagdeev, K. Hun Mok, P. J. Hore, J. Phys. Chem. B 2004, 108, 15355-15363.

F. E. Neumann, J. Math. Pures Appl. 1848, 13, 113-178.

M. P. Nicholas, E. Eryilmaz, F. Ferrage, D. Cowburn, R. Ghose, Prog. Nucl. Mag. Res. Sp. 2010, 57, 111-158.

T. M. Nordlund, X. Y. Liu, J. H. Sommer, Proc. Natl. Acad. Sci. U.S.A. 1986, 83, 8977-8981.

R. M. Noyes, J. Chem. Phys. 1954, 22, 1349-1359.

M. Ottiger, F. Delaglio, A. Bax, J. Magn. Reson. 1998, 131, 373-378.

M. Ottiger, F. Delaglio, J. L. Marquardt, N. Tjandra, A. Bax, J. Magn. Reson. 1998, 134, 365-369.

G. Otting, J. Biomol. NMR 2008, 42, 1-9.

G. Otting, Annu. Rev. Biophys. 2010, 39, 387-405.

J. B. Pedersen, J. H. Freed, J. Chem. Phys. 1973, 58, 2746-2762.

J. B. Pedersen, J. H. Freed, J. Chem. Phys. 1973, 59, 2869-2885.

J. B. Pedersen, J. H. Freed, J. Chem. Phys. 1974, 61, 1517-1526.

J. B. Pedersen, J. H. Freed, J. Chem. Phys. 1975, 62, 1706-1711.

F. Peters, A. Maestre-Martinez, M. Leonov, L. Kovacic, S. Becker, R. Boelens, C. Griesinger, J. Biomol. NMR 2011, 51, 329-337.

G. Porcal, S. G. Bertolotti, C. M. Previtali, M. V. Encinas, Phys. Chem. Chem. Phys. 2003, 5, 4123-4128. 
[Prudencio2004]

[Purcell1946]

[Ranganathan2002a]

[Ranganathan2002b]

[Reese2005]

[Roberts 1993]

[Rodriguez2006]

[Roth1974]

[Rueckert2000]

[Sass2000]

[Saupe1968]

[Sayah2011]

[Schmidt2012a]

[Sekhar2009]

[Siepel2009]

[Smirnova2006]
M. Prudêncio, J. Rohovec, J. A. Peters, E. Tocheva, M. J. Boulanger, M. E. P. Murphy, H. J. Hupkes, W. Kosters, A. Impagliazzo, M. Ubbink, Chem. Eur. J. 2004, 10, 3252-3260.

E. M. Purcell, H. C. Torrey, R. V. Pound, Phys. Rev. 1946, 69, 37-38.

R. S. Ranganathan, R. K. Pillai, N. Raju, H. Fan, H. Nguyen, M. F. Tweedle, J. F. Desreux, V. Jacques, Inorg. Chem. 2002, 41, 6846-6855.

R. S. Ranganathan, N. Raju, H. Fan, X. Zhang, M. F. Tweedle, J. F. Desreux, V. Jacques, Inorg. Chem. 2002, 41, 6856-6866.

C. B. Reese, Org. Biomol. Chem. 2005, 3, 3581-3868.

G. C. K. Roberts, NMR of Macromolecules, Oxford University Press, New York, 1993.

F. Rodriguez-Castañeda, P. Haberz, A. Leonov, C. Griesinger, Magn. Reson. Chem. 2006, 44, 10-16.

H. D. Roth, A. A. Lamola, J. Am. Chem. Soc. 1974, 96, 6270-6275.

M. Rueckert, G. Otting, J. Am. Chem. Soc. 2000, 122, 7793-7797.

H. J. Sass, G. Musco, S. J. Stahl, P. T. Wingfield, S. Grzesiek, J. Biomol. NMR 2000, 18, 303-309.

A. Saupe, Angew. Chem., Int. Ed. 1968, 7, 97-112.

M. Sayah, M. G. Organ, Chem. Eur. J. 2011, 17, 11719-11722.

M. Schmidt, H. Sun, A. Leonov, C. Griesinger, U. M. Reinscheid, Magn. Reson. Chem. 2012, 50, 38-44.

A. Sekhar, S. Cavagnero, J. Phys. Chem. B 2009, 113, 8310-8318.

F. Siepel, Click-Reaktion zur paramagnetischen Markierung von DNA und Strukturaufklärung mittels NMR-Spektroskopie, Diplomarbeit, Göttingen, 2009.

N. N. Smirnova, T. A. Bykova, B. Van Durme, K. van Van Mele, J. Chem. Thermodyn. 2006, 38, 879-883. 
[Solomon1955]

[Song2011]

[Sonogashira1975]

[Sonogashira2002]

[Su2008]

[Su2010]

[Swarbrick2011]

[Thiele2003]

[Tjandra1996a]

[Tolman1995]

[Tsentalovich2002]

[Tycko2000]

[vBerg2001]

[Viguier2001]

[Vlasie2007]

[Vollenweider1985]

[Vollenweider1988]
I. Solomon, Phys. Rev. 1955, 99, 559-566.

Y. Song, T. J. Meade, A. V. Astashkin, E. L. Klein, J. H. Enemark, A. Raitsimring, J. Magn. Reson. 2011, 210, 59-68.

K. Sonogashira, Y. Tohda, N. Hagihara, Tetrahedron Lett. 1975, 50, 44674470.

K. Sonogashira, J. Organomet. Chem. 2002, 653, 46-49.

X. C. Su, B. Man, S. Beeren, H. Liang, S. Simonson, C. Schmitz, T. Huber, B. A. Messerle, G. Otting, J. Am. Chem. Soc. 2008, 130, 10486-10487.

X. C. Su, G. Otting, J. Biomol. NMR 2010, 46, 101-112.

J. D. Swarbrick, P. Ung, S. Chhabra, B. Graham, Angew. Chem., Int. Ed. 2011, 50, 4403-4406.

C. M. Thiele, S. Berger, Org. Lett. 2003, 5, 705-708.

N. Tjandra, S. Grzesiek, A. Bax, J. Am. Chem. Soc. 1996, 118, 6264-6272.

J. R. Tolman, J. M. Flanagan, M. A. Kennedy, J. H. Prestegard, Proc. Natl. Acad. Sci. U.S.A. 1995, 92, 9279-9283.

Y. P. Tsentalovich, J. J. Lopez, P. J. Hore, R. Z. Sagdeev, Spectrochim. Acta, Part A 2002, 58, 2043-2050.

R. Tycko, F. J. Blanco, Y. Ishii, J. Am. Chem. Soc. 2000, 122, 9340-9341.

P. A. W. van den Berg, J. Widengren, M. A. Hink, R. Rigler, A. J. W. G. Visser, Spectrochim. Acta, Part A 2001, 57, 2135-2144.

R. Viguier, G. Serratrice, A. Dupraz, C. Dupuy, Eur. J. Inorg. Chem. 2001, 2001, 1789-1795.

M. D. Vlasie, C. Comuzzi, A. M. C. H. van den Nieuwendijk, M. Prudêncio, M. Overhand, M. Ubbink, Chem. Eur. J. 2007, 13, 1715-1723.

J. K. Vollenweider, H. Fischer, J. Hennig, R. Leuschner, Chem. Phys. 1985, 97, 217-234.

J. K. Vollenweider, H. Fischer, Chem. Phys. 1988, 124, 333-345. 
[Ward1967]

[Wei2013]

[Werbelow1979]

[Woehnert2003]

[Woeltjen2009]

[Yagi2010]

[Yang2013]

[Yi2011]

[Zhang2006]

[Zhang2007]
H. R. Ward, R. G. Lawler, J. Am. Chem. Soc. 1967, 89, 5518-5519.

Z. Wei, Y. Yang, Q. F. Li, F. Huang, H. H. Zuo, X. C. Su, Chem. Eur. J. 2013, 19, 5758-5764.

L. G. Werbelow, J. Chem. Phys. 1979, 70, 5381-8383.

J. Woehnert, K. J. Franz, M. Nitz, B. Imperiali, H. Schwalbe, J. Am. Chem. Soc. 2003, 125, 13338-13339.

E. Wöltjen, Paramagnetisch markierte Oligonukleotide, PhD thesis, Goettingen, 2009.

H. Yagi, K. V. Loscha, X. C. Su, M. Stanton-Cook, T. Huber, G. Otting, J. Biomol. NMR 2010, 47, 143-153.

Y. Yang, Q. F. Li, C. Cao, F. Huang, X. C. Su, Chem. Eur. J. 2013, 19, 1097-1103.

J. Yi, Y. Fu, B. Xiao, W. C. Cui, Q. X. Guo, Tetrahedron Lett. 2011, 52, 205-208.

Q. Zhang, X. Sun, E. D. Watt, H. M. Al-Hashimi, Science (Washington, DC, U. S.) 2006, 311, 653-656.

Q. Zhang, A. C. Stelzer, C. K. Fisher, H. M. Al-Hashimi, Nature (London, U. K.) 2007, 450, 1263-1267. 


\section{List Of Abbreviations}

\begin{tabular}{|c|c|}
\hline AA & Ammonium acetate \\
\hline Ac & Acetyl \\
\hline Asc & Ascorbate \\
\hline $\mathrm{Bu}$ & Butyl \\
\hline CIDNP & Chemically induced dynamic nuclear polarization \\
\hline CLaNP & Caged lanthanide NMR probe \\
\hline CSA & Chemical shift anisotropy \\
\hline DCM & Dichloromethane \\
\hline DIPEA & $N, N$-Diisopropylethylamine \\
\hline DMAP & 4-Dimethylaminopyridine \\
\hline DMSO & Dimethyl sulfoxide \\
\hline DMT & Dimethoxytrityl \\
\hline DNA & Desoxyribonucleic acid \\
\hline DOTA & $1,4,7,10$-Tetraazacyclododecane- $N, N^{\prime}, N^{\prime \prime}, N^{\prime \prime \prime}$-tetraacetic acid \\
\hline DTBPA & Dithiobis(phenylazide) \\
\hline EDTA & Ethylenediaminetetraacetic acid \\
\hline Et & Ethyl \\
\hline F-tyr & 3-Fluoro-L-tyrosine \\
\hline FMN & Flavin mononucleotide \\
\hline HPLC & High performance liquid chromatography \\
\hline
\end{tabular}




\begin{tabular}{|c|c|}
\hline HSQC & Heteronuclear single quantum coherence \\
\hline IPAP & In phase - anti phase \\
\hline $\mathrm{Me}$ & Methyl \\
\hline MOPS & 3-(N-morpholino)propanesulfonic acid \\
\hline MWCO & Molecular weight cut off \\
\hline NaAsc & Sodium ascorbate \\
\hline NMR & Nuclear magnetic resonance spectroscopy \\
\hline NOESY & Nuclear Overhauser effect spectroscopy \\
\hline PCS & Pseudocontact shifts \\
\hline photo CIDNP & Photochemically induced dynamic nuclear polarization \\
\hline ppm & Parts per million \\
\hline PRE & Paramagnetic relaxation enhancement \\
\hline RCSA & Residual chemical shift anisotropy \\
\hline RDC & Residual dipolar coupling \\
\hline RMSD & Root-mean-square deviation \\
\hline RNA & Ribonucleic acid \\
\hline TBTA & Tris[(1-benzyl-1H-1,2,3-triazol-4-yl)methyl]amine \\
\hline TCEP & Tris(2-carboxyethyl)phosphine hydrochloride \\
\hline TEAA & Triethylammonium acetate \\
\hline TEDA & Triethylenediamine \\
\hline THF & Tetrahydrofuran \\
\hline
\end{tabular}


TMS Trimethylsilane

TPTA Tris[[1-(3-hydroxypropyl)-1H-1,2,3-triazol-4-yl]methyl]amine

Tyr L-Tyrosine

UV Ultraviolet 



\section{List Of Figures}

$1.1 S$-(2-Pyridylthio)cysteaminyl ethylenediamine tetraacetic acid. . . . . . . . 3

1.2 Structure of CLaNP-1. . . . . . . . . . . . . . . . . 3

1.3 Structures of the two enantiomers of the second generation EDTA based tags. . . . . 4

1.4 Structures of CLaNP-3, CLaNP-5.1 and CLaNP-5.2. . . . . . . . . . 5

1.5 Structure of the DOTA-M8 tag. . . . . . . . . . . . . . . 6

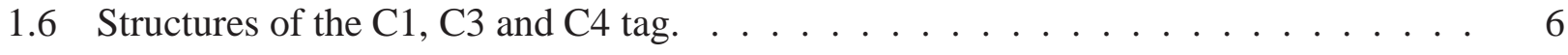

1.7 Structures of DPA, 4MMDPA, 3MDPA and 4MDPA . . . . . . . . . . 7

1.8 Structure of the iminodiacetic acid based tag. . . . . . . . . . . . 8

1.9 The Cys-Ph-TAHA tag (cysteine-phenyl-triaminohexaacetic acid) . . . . . . . . 8

1.10 The caged lanthanide NMR probe, CLaNP-7. . . . . . . . . . . 9

1.11 Structures of the two thiol-ene binding tags 4VDPA and 4VPyMTA . . . . . . . 9

1.12 Structures of the five nucleobases. . . . . . . . . . . . . . . . 10

1.13 Demonstration of the photo CIDNP effect for 3-fluoro-L-tyrosine. . . . . . . . . . 11

2.1 The vector between two magnetic moments in an arbitrary reference frame. . . . . 18

2.2 Definition of the angle between the external magnetic field and two magnetic moments. 19

2.3 The external magnetic field and the vector between two magnetic moments in an arbitrary coordinate system. . . . . . . . . . . . . . . . 20

2.4 Examples for a probability matrix (left) and an alignment tensor (right). . . . . . 22

2.5 Definition of the angles for the Saupe matrix. . . . . . . . . . . . 27

2.6 Electronic states and allowed transitions for a photoactive substance. $A=$ absorbance, $F=$ fluorescence, $P=$ phosphorescence, ISC $=$ intersystem crossing. $\ldots \ldots \ldots$

2.7 The distance dependency of the energies of the four electronic states. . . . . . . . . 54

2.8 1: Radical pair in the triplet state. 2: Seperation. 3: Larmor precession. 4: Spatial

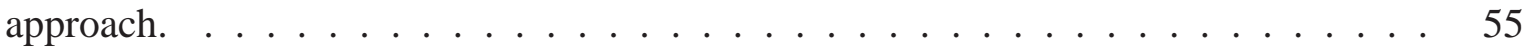

2.9 The effect of the hyperfine interaction on the intersystem crossing rate. In this example the hyperfine coupling increases the Larmor frequency of the $\alpha$-state. . . . . . . 57

2.10 The predicted signal forms of Kaptein's multiplet effect. . . . . . . . . . . . . 59

3.1 Schematic drawing of the photo CIDNP setup. . . . . . . . . . . . . 61 
3.2 The signal of the $\mathrm{CH}_{2}$ group of ethanol with (red) and without (black) the coaxial insert and fiber.

3.3 Pulse sequence used in all photo CIDNP experiments. An arbitrarily long laser pulse was followed by the mixing time and data aquisition was done directly after a $90^{\circ}$ NMR pulse.

3.4 25: Flavin mononucleotide. 26: 3-Fluoro-L-tyrosine. 27: L-Tyrosine. 28: Triethylenediamine.

3.5 The photocycle for the reaction of FMN with F-tyr.

3.6 Example of the TEDA signal. Magenta: Dark spectrum. Dark Blue: Light spectrum (10 ms laser pulse). Green: Light spectrum (20 ms laser pulse)

3.7 Determination of the longitudinal relaxation time after a $10 \mathrm{~ms}$ laser pulse. . . . . 67

3.8 Determination of the longitudinal relaxation time with a standard inversion recovery experiment.

3.9 Average enhancement of the TEDA signal for three different temperatures. . . . . . 68

3.10 Comparison of the F-tyr dark and light spectrum after a $100 \mathrm{~ms}$ laser pulse, $100 \mathrm{~ms}$ mixing time and a $90^{\circ} \mathrm{NMR}$ pulse. Shown are the aromatic and beta protons.

3.11 The fluorine signal intensity of F-tyr for different laser pulse duration. The initial buildup rate was determined to $78 \mathrm{~s}^{-1}$ (red line) . . . . . . . . . . .

3.12 The observed aromatic H2 signal of F-tyr, after a $100 \mathrm{~ms}$ laser pulse, dependent on the mixing time.

3.13 The observed relative $\mathrm{H} 2$ signal intensity of F-tyr after a $100 \mathrm{~ms}$ laser pulse, dependent on the mixing time.

3.14 The observed relative F3 signal intensity of F-tyr after a $100 \mathrm{~ms}$ laser pulse, dependent on the mixing time.

3.15 The calculated relative $2 H_{z}^{(2)} F_{z}$ signal intensity of F-tyr after a $100 \mathrm{~ms}$ laser pulse, dependent on the mixing time.

3.16 The $\beta$-protons of tyrosine were enhanced by a factor of 4.6 for the first experiment.

3.17 Enhancement factors of the three proton groups of tyrosine for three consecutive experiments. 
3.18 The aromatic region of F-tyr after every 10 scans with a $100 \mathrm{~ms}$ laser pulse, $50 \mathrm{~ms}$ mixing time and 5 min relaxation delay.

3.19 Comparison of the experimental data (black squares) for an inversion recovery experiment and the simluated data for the same starting magnetization (black lines) for F3 (left) and $\mathrm{H} 2$ (right). . . . . . . . . . . . . . . . . . . . . . . .

4.1 Cys-Ph-TAHA tag coordinating $\mathrm{Tb}^{3+} \ldots \ldots \ldots \ldots \ldots$

4.2 The sequence of the test DNA strand. The X marks the position of the modification. In the wildtype DNA, a thymidine occupies this position.

4.3 Catalytic cycle of the 1,3 -dipolar cycloaddition. . . . . . . . . . . 84

4.4 The two triazolyl ligands used in this work. . . . . . . . . . . . . . . 84

4.5 The reaction pathway to the final nucleoside containing a carbon triple bond. . . . . 85

4.6 The modified tag for the cycloaddition, Azide-Ph-TAHA . . . . . . . . . 86

4.7 Overlay of the spectra for the lutetium loaded Azide-Ph-TAHA tag (black) and the unloaded tag (red). The numbers above the signals indicate the relative integral intensities.

4.8 Section of the ${ }^{1} \mathrm{H}^{13}{ }^{13} \mathrm{C}-\mathrm{HSQC}$ spectrum of the lutetium loaded tag. . . . . . . . . . 88

4.9 Cycloaddition to introduce a sulfur moiety into a DNA strand. . . . . . . . . . . 91

4.10 Structure of the first Cys-Ph-TAHA tagged DNA loaded with a lanthanide ion. . . . . 92

4.11 The tested exchange of the bromine with a sulfur. . . . . . . . . . . . 93

4.12 Mass spectra of the DNA strands with DTBPA (left) and with the free thiol moiety (right).

4.13 NOESY walk region of the ${ }^{1} \mathrm{H}-{ }^{1} \mathrm{H}-\mathrm{NOESY}$ spectrum of the lutetium loaded DNA. . .

4.14 Schematic representation of the principle behind ${ }^{1} \mathrm{H}-{ }^{13} \mathrm{C}-\mathrm{HSQC}-I P A P$ spectra. . . . .

4.15 Mass spectra of the three tagged DNA strand: Tb (left), Lu (center) and Tm (right). .

4.16 The aromatic region of diamagnetic (Lu: red) and paramagnetic (Tb: black) DNA. The spectra are scaled for comparability. . . . . . . . . . . . . . . . 100

4.17 Aromatic region and ammonium acetate signal of Tb-loaded DNA. 1. Blue: After tagging and one HPLC purification run. 2. Black: After 1. and one week of lyophilization. 3. Red: After 2. and a second HPLC purification sun. . . . . . . . . . 
4.18 Aromatic region of Tb loaded DNA after HPLC purification (black) and after washing with $1 \mathrm{~mol} / \mathrm{L} \mathrm{NaCl}$ solution $(12 \times 20 \mathrm{~mL})($ red) $\ldots \ldots \ldots \ldots$. . . . . . . . 102

4.19 Aromatic region of Tb loaded DNA several times after preparation of the sample. . . 103

4.20 Combination of disulfide binding and cycloaddition yielded Cys-Ph-TAHA-tagged

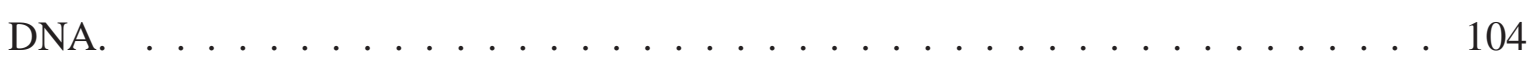

4.21 Elongation and binding of tagged DNA to TAR-RNA of HIV-1 . . . . . . . . . 105 


\section{Appendix}

\subsection{HSQC-IPAP Pulse Program}

\section{;HSQCPR_IPAP}

;G. Bodenhausen and D.J. Ruben, Chem. Phys. Lett. 69, 185 (1980)

;D.R. Muhandiram et al, JMR B102, 317-321 (1993)

;M. Ottinger et al, JMR, 373-378 (1998)

;pl1 : power for $1 \mathrm{H}$

;p12 : power for $13 \mathrm{C}$ hard

;pl12 : power for 13C GARP decoupling

;p1 : 90 degree hard pulse $1 \mathrm{H}$

;p3 : 90 degree hard pulse 13C

;p4 : 13C pulse, 225deg for 500/600, 180deg for $750 / 900$

;pcpd2 : 90 deg cpd-pulse 13C ( 85us)

;p20 : 1m (Gradient before first INEPT)

;p21 : 1m (Gradient in first INEPT)

;p22 : 800u (Gradient in first INEPT)

;p23 : $1 \mathrm{~m}$ (Gradient for $\mathrm{z}$-filter)

;p24 : 1m (Gradient for second INEPT)

;p25 : 500u (Gradient for IP AP conversion INEPT)

;gpz0 : $80 \%$

;gpz1 : $19 \%$

;gpz2 : $30 \%$

;gpz3 : $65 \%$

;gpz4 : $15 \%$

;gpz5 : $17 \%$

$; \mathrm{d} 1$ : relaxation delay

;d2 : 1H-13C INEPT delay $(1.7 \mathrm{~m})$

;in $0: 1 / 2 * \mathrm{SW}($ in $\mathrm{Hz})$

\#include <Avance.incl>

define delay INEPT_1

define delay INEPT_2

define delay INEPT_3

\#define GRADIENT0 10u p20:gp0 200u

\#define GRADIENT1 10u p21:gp1 200u

\#define GRADIENT2 10u p22:gp2 200u

\#define GRADIENT3 10u p23:gp3 200u

\#define GRADIENT4 10u p24:gp4 200u

\#define GRADIENT5 10u p25:gp5 200u

"p2=p1*2"

"in0=inf1/2" 
"d0=in0/2-p3*2/3.14159"

"INEPT_1=d2-(p21+210u)"

"INEPT_2=d2-(p24+210u)"

"INEPT_3=d2-(p25+210u)"

"13=td1/4"

$110 \mathrm{u} z \mathrm{ze}$

;--IP-part

$1840 u$

$1910 \mathrm{u}$

$201 \mathrm{~m}$

$301 \mathrm{~m}$ do:f2

20u pl9:f1

10u LOCKH_OFF

d1 cw:f1 ph29

4u do:f1

10u LOCKH_ON

20u pl1:f1

20u pl2:f2

(p3 ph20):f2

GRADIENT0

$10 \mathrm{~m}$

; - first INEPT

(p1 ph20):f1

GRADIENT1

INEPT_1

(center (p2 ph20):f1 (p3 ph20 2u p4 ph21 2u p3 ph20):f2)

GRADIENT1

INEPT_1

(p1 ph2):f1

GRADIENT2

;-13C evolution

(p3 ph1 d0 d0 p3 ph20):f2

GRADIENT3

(p1 ph20):f1

; - second INEPT

GRADIENT4

INEPT_2

(center (p2 ph20):f1 (p3 ph20 2u p4 ph21 2u p3 ph20):f2)

GRADIENT4

INEPT_2 pl12:f2 LOCKH_OFF

;_—acquisition of IP-part

go=18 ph31 cpd2:f2

$1 \mathrm{~m} \mathrm{do:f2} \mathrm{wr} \# 0$ if \#0 zd 


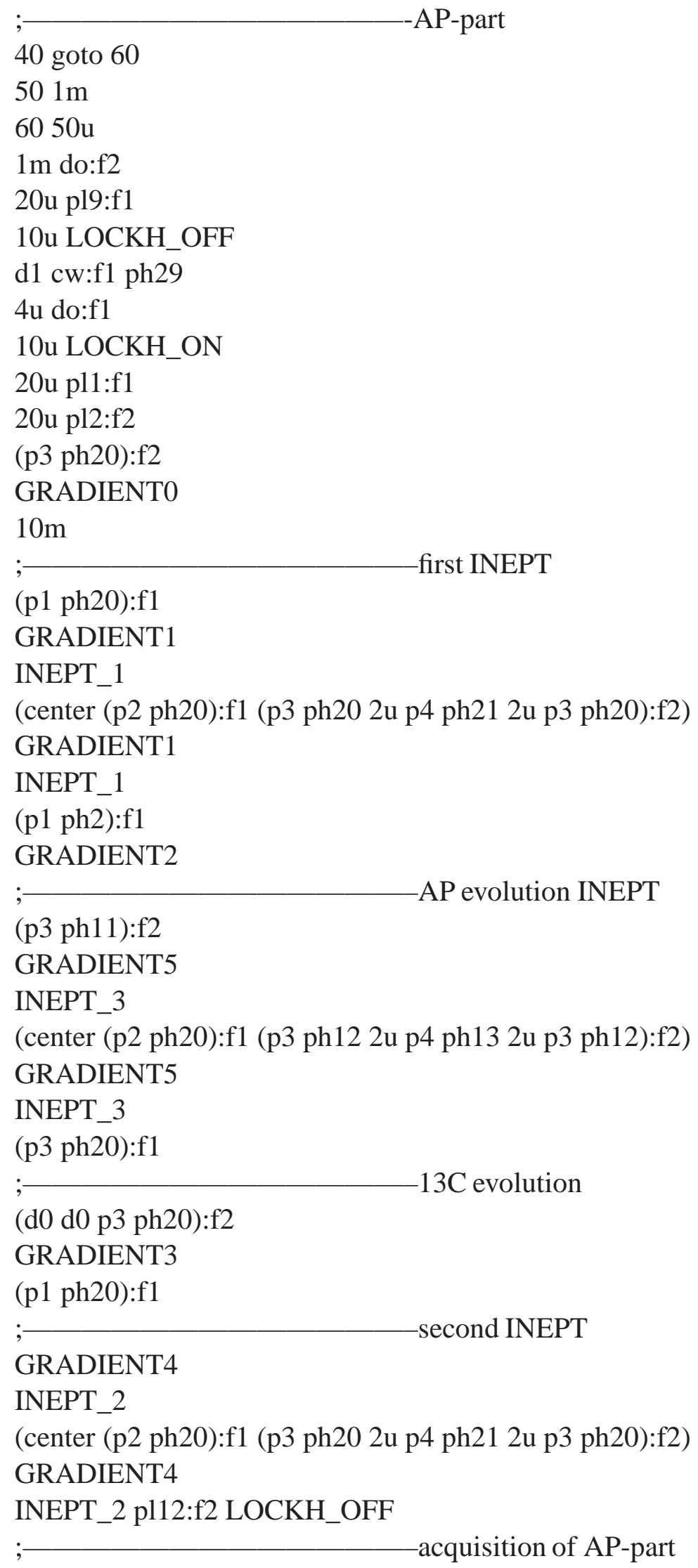


go=50 ph31 cpd2:f2

$1 \mathrm{~m}$ do:f2 wr \#0 if \#0 zd

10u ip1

10u ip11

10u ip12

10u ip13

lo to 19 times 2

;- for frequency descrimination

$10 \mathrm{u}$ id0

lo to 20 times 13

;- $13=\mathrm{td} 1 / 2$

10u LOCKH_OFF

exit

ph1 $=02$

ph2=1 133

ph11=13

ph12 $=0$

ph13=1

$\operatorname{ph} 20=0$

$\operatorname{ph} 21=1$

$\operatorname{ph} 22=2$

$\operatorname{ph} 23=3$

$\mathrm{ph} 29=0$

ph31=2 0002

\subsection{D Pulse Program With Laser Trigger}

;edit of zg for laser trigger

;avance-version (06/11/09)

;1D sequence

;\$CLASS=HighRes

;\$DIM=1D

;\$TYPE=

;\$SUBTYPE=

;\$COMMENT=

\#include <Avance.incl>

"acqt $0=-p 1 * 2 / 3.1416 "$

"d12=20u"

$1 \mathrm{ze}$

setnmr3|12

d19

$230 \mathrm{~m}$ 


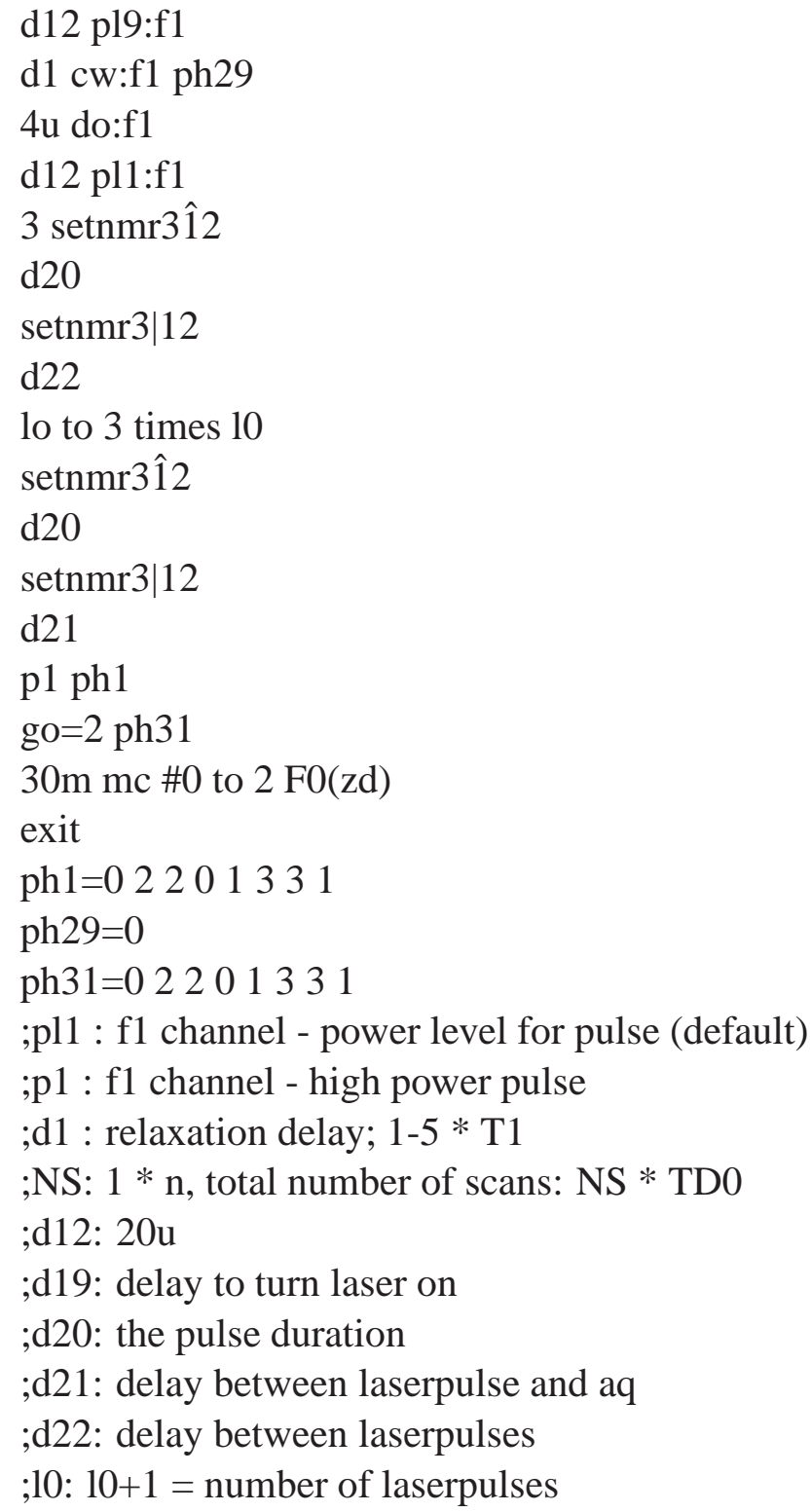

\subsection{Mathematica Monte - Carlo Script}

Needs["DifferentialEquations 'NDSolveProblems"]

Needs["DifferentialEquations 'NDSolveUtilities"]

ClearAll[inpH, inpF, inpFH]; (* experimental data input*)

inpH="time, integral value";

inpF="time, integral value";

inpFH="time, integral value";

ClearAll[GammaH, GammaF, H, F]; (* define the differences in the equlibrium polarization *)

$\mathrm{GammaH}=26.752 * 10^{7}$;

$\mathrm{GammaF}=25.17 * 10^{7}$; 
$\mathrm{H}=\mathrm{GammaH} / \mathrm{GammaF}$;

$\mathrm{F}=\mathrm{GammaF} / \mathrm{GammaH}$;

ClearAll[z, results];

results $=\{\}$;

$\mathrm{z}=100 ;(*$ number of iterations * $)$

Do[(

ClearAll[err, resint];

err $=0.05$;

resint $=($ ClearAll[model, i, s, hf, timeH, timeF, timeFH, fitH, fitF, fitFH, intH, intF, intFH, errH, errF, errFH, errtot, rhoHH, sigmaHF, rhoFF, deltaFHF, rhoHFHF, inpHMC, inpFMC, inpFHMC, m, k, 1 , $\mathrm{n}, \mathrm{o}]$;

model[rhoHH_? NumericQ, sigmaHF_?NumericQ, rhoFF_?NumericQ, deltaFHF_?NumericQ, rhoHFHF_ ?NumericQ] $:=\left(\operatorname{model}\left[\mathrm{rhoHH}\right.\right.$, sigmaHF, rhoFF, deltaFHF, rhoHFHF] $=$ NDSolve[ $\left\{\mathrm{i}^{\prime}[\mathrm{t}]\right.$ $==-\operatorname{rhoHH}(\mathrm{i}[\mathrm{t}]-1)-\operatorname{sigmaHF}(\mathrm{F} \mathrm{s}[\mathrm{t}]-\mathrm{F} 1), \mathrm{s}^{\prime}[\mathrm{t}]==-\operatorname{rhoFF}(\mathrm{F} \mathrm{s}[\mathrm{t}]-\mathrm{F} 1)-$

sigmaHF(i[t] - 1) - deltaFHF hf $[\mathrm{t}]$, hf' $[\mathrm{t}]==-\operatorname{deltaFHF}(\mathrm{F} \mathrm{s}[\mathrm{t}]-\mathrm{F} 1)-\operatorname{rhoHFHF}$ hf $[\mathrm{t}]$, $\left.\mathrm{i}[0.05]==1.5784, \mathrm{~s}[0.2]==\mathrm{F}^{*} 25.4372, \mathrm{hf}[0.1]==-1.3147\right\},\{\mathrm{i}, \mathrm{s}, \mathrm{hf}\},\{\mathrm{t}, 0,40\}$,

Method - >"ExplicitRungeKutta"]); (* define a model for the coupled differential equations *) inpHMC=inpH; $(*$ rename the original input files *)

inpFMC=inpF;

inpFHMC=inpFH;

For $[\mathrm{m}=1, \mathrm{~m}<12$, inpHMC[[m, 2]]=Random[NormalDistribution[inpHMC[[m, 2]], Abs[inpHMC[[m, $2]]^{*}$ err ]]], $\left.\mathrm{m}++\right] ;(*$ create new values within the standard deviation *)

For[o=1, o<10, inpFMC[[o, 2]]=Random[NormalDistribution[inpFMC[[o, 2]], Abs[inpFMC[[o, 2]]* err]]], $\mathrm{O}++]$;

For[n=1, n<10, inpFHMC[[n, 2]]=Random[NormalDistribution[inpFHMC[[n, 2]],

Abs[inpFHMC[[n, 2]]* err ]]], n++];

timeH=Table[inpH[[k, 1]], \{k, Length[inpH] $\}] ;(*$ extract the timevalues of the data points * $)$

timeF=Table[inpF[[k, 1]], \{k, Length[inpF] $\}]$;

timeFH=Table[inpFH[[k, 1]], \{k, Length[inpFH] $\}]$;

intH :=Table[inpHMC[[k, 2]], \{k, Length[inpH]\}]; (* get the integral values *)

intF :=Table[inpFMC $[[\mathrm{k}, 2]]$, $\{\mathrm{k}$, Length[inpF] $\}]$;

intFH :=Table[inpFHMC [[k, 2]], \{k, Length[inpFH] $\}]$;

fitH[rhoHH_? NumericQ, sigmaHF_?NumericQ, rhoFF_?NumericQ, deltaFHF_?NumericQ,

rhoHFHF_?NumericQ] :=i[timeH]/.model[rhoHH, sigmaHF, rhoFF, deltaFHF, rhoHFHF];

(* fit the equations to the graph *)

fitF[rhoHH_?NumericQ, sigmaHF_?NumericQ, rhoFF_? NumericQ, deltaFHF_? NumericQ, rhoHFHF_ ?NumericQ] :=s[timeF]/.model[rhoHH, sigmaHF, rhoFF, deltaFHF, rhoHFHF]; fitFH[rhoHH_?NumericQ, sigmaHF_?NumericQ, rhoFF_?NumericQ, deltaFHF_? NumericQ, rhoHFHF_?NumericQ] :=hf[timeFH]/model[rhoHH, sigmaHF, rhoFF, deltaFHF, rhoHFHF]; errH[rhoHH_?NumericQ, sigmaHF_?NumericQ, rhoFF_? NumericQ, deltaFHF_? NumericQ, rhoHFHF_?NumericQ] :=Sqrt[Sum[(fitH[rhoHH, sigmaHF, rhoFF, deltaFHF, rhoHFHF][[1, 1]] - intH[[1]] $)^{2},\{1$, Length[inpH] $\left.\}\right] /$ Length[inpH]];

(* calculate the error between the fit and the graph *) 
errF[rhoHH_? NumericQ, sigmaHF_?NumericQ, rhoFF_?NumericQ, deltaFHF_? NumericQ, rhoHFHF_?NumericQ] :=Sqrt[Sum[(fitF[rhoHH, sigmaHF, rhoFF, deltaFHF, rhoHFHF][[1, 1]] - intF[[1]] $)^{2},\{1$, Length[inpF] $\left.\}\right] /$ Length[inpF]];

errFH[rhoHH_? NumericQ, sigmaHF_?NumericQ, rhoFF_?NumericQ, deltaFHF_?NumericQ, rhoHFHF_?NumericQ] :=Sqrt[Sum[(fitFH[rhoHH, sigmaHF, rhoFF, deltaFHF, rhoHFHF][[1, 1]] - intFH[[1]] $)^{2},\{1$, Length[inpFH] $\left.\}\right] /$ Length[inpFH]];

errtot[rhoHH_?NumericQ, sigmaHF_?NumericQ, rhoFF_?NumericQ, deltaFHF_? NumericQ, rhoHFHF_ ?NumericQ] :=(errH[rhoHH, sigmaHF, rhoFF, deltaFHF, rhoHFHF]+errF[rhoHH, sigmaHF, rhoFF, deltaFHF, rhoHFHF]+errFH[rhoHH, sigmaHF, rhoFF, deltaFHF, rhoHFHF]); NMinimize[errtot[rhoHH, sigmaHF, rhoFF, deltaFHF, rhoHFHF], \{rhoHH, sigmaHF, rhoFF, deltaFHF, rhoHFHF\}, Method - >"DifferentialEvolution"] (* minimize the error by adjusting the relaxation rates *)); AppendTo[results, resint[[2, All, 2]]]; )$,\{z\}]$ 



\section{Curriculum Vitae}

\section{Personal data}

Name

Birtday and birthplace

Citizenship
Florian Siepel

18.10.1982, Neustadt a. Rbge, Germany

German

School and academic education

1989-1993 Primary school in Otternhagen

1993-1999 Middle school Leineschule in Neustadt a. Rgbe

1999-2002 Training as a banker at Sparkasse Hannover

2002-2005 Secondary school Gaußstraße in Neustadt a. Rbge

2005-2009 Academic studies of chemistry at the University of Göttingen and diploma thesis at the Max Planck Institute for Biophysical Chemistry in Göttingen with the title Click-Reaktion zur paramagnetischen Markierung von DNA und Strukturaufklärung mittels NMR-Spektroskopie, principle investigator Prof. Dr. C. Griesinger

2010-2013 Doctoral thesis at the Max Planck Institute for Biophysical Chemistry in Göttingen with the thesis title: The Advantages Of Paramagnetic NMR, principle investigator Prof. Dr. C. Griesinger

\section{Scholarships}

2010-2012 Excellence stipend of the International Max Planck Research School

2013 Student travel stipend for the 54th ENC conference in Pacific Grove, CA, USA

Göttingen, September 2013 